\title{
Reactor-Specific Spent Fuel Discharge Projections: 1985 to 2020
}

\author{
C. M. Heeb \\ R. A. Libby \\ R. C. Walling \\ W. L. Purcell
}

September 1986

Prepared for the U.S. Department of Energy under Contract DE-AC06-76RLO 1830

Pacific Northwest Laboratory Operated for the U.S. Department of Energy by Battelle Memorial Institute 


\title{
DISCLAIMER
}

This report was prepared as an account of work spons.ored by an agency of the United States Government. Neither the United States Government nor any agency thereof, nor any of their employees, makes any warranty, express or implied, or assumes any legal liability or responsibilicy for the accuracy, completeness, or usefulness of any information, apparatus, product, or process disclosed, or represents that its use would not infringe privately owned rights. Reference herein to any specific commercial product, process, or service by trade name, trademark, manufacturer, or otherwise, does not necessarily constitute or imply its endorsement, recommendation, or favoring by the United States Government or any agency thereof. The views and opinions of authors expressed herein do not necessarily state or reflect those of the United States Government or any agency thereof.

\author{
PACIFIC NORTHWEST LABORA.TORY \\ operated by \\ BATTELLE \\ for the \\ UNITED STATES DEPARTMENT OF ENERGY \\ under Contract DE-AC06-76RLC) 1830
}

\begin{tabular}{|c|c|}
\hline \multirow{2}{*}{\multicolumn{2}{|c|}{ Printed in the United States of America }} \\
\hline & \\
\hline \multirow{2}{*}{\multicolumn{2}{|c|}{$\begin{array}{c}\text { Available from } \\
\text { National Technical Information Service }\end{array}$}} \\
\hline & \\
\hline \multirow{3}{*}{\multicolumn{2}{|c|}{$\begin{array}{c}\text { United States Department of Commerce } \\
5285 \text { Port Royal Road } \\
\text { Springfield, Virginia } 22161\end{array}$}} \\
\hline & \\
\hline & \\
\hline \multirow{2}{*}{\multicolumn{2}{|c|}{$\begin{array}{l}\text { NTIS Price Codes } \\
\text { Microfiche A01 }\end{array}$}} \\
\hline & \\
\hline \multicolumn{2}{|c|}{ Printed Copy } \\
\hline & Price \\
\hline Pdges & Codes \\
\hline $001-025$ & $\mathrm{~A} 02$ \\
\hline $026-050$ & $\mathrm{~A} 03$ \\
\hline $051-075$ & $\mathrm{~A} 04$ \\
\hline $076-100$ & A05 \\
\hline $101-125$ & $\mathrm{~A} 06$ \\
\hline $126-150$ & $\mathrm{~A} 07$ \\
\hline $151-175$ & $A 0 B$ \\
\hline $176-200$ & $\mathrm{~A} 09$ \\
\hline $201-225$ & $A 010$ \\
\hline $226-250$ & $A 011$ \\
\hline $251-275$ & $\mathrm{~A} 012$ \\
\hline $276-300$ & $A 013$ \\
\hline
\end{tabular}



C. M. Heeb
R. A. Libby
R. C. Walling
W. L. Purcel1

September 1986

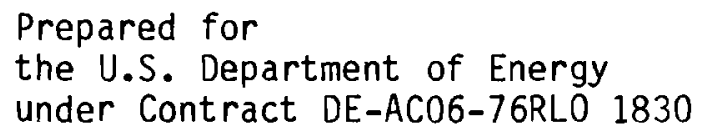

Pacific Northwest Laboratory

Richland, Washington 99352 


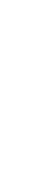


The creation of four spent-fuel data bases that contain information on the projected amounts of spent fuel to be discharged from U.S. commercial nuclear reactors through the year 2020 is described. The data bases contain detailed spent-fuel information from existing, planned, and projected pressurized water reactors (PWR) and boiling water reactors (BWR). The projections are based on individual reactor information supplied by the U.S. reactor owners. The basic information is adjusted to conform to Energy Information Agency (EIA) forecasts for nuclear installed capacity, generation, and spent fuel discharged. The EIA cases considered are: 1) No New Orders with Extended Burnup, 2) No New Orders with Constant Burnup, 3) Middle Case with Extended Burnup, and 4) Middle Case with Constant Burnup. Detailed, by-reactor tables are provided for annual discharged amounts of spent fuel, for storage requirements assuming maximum-atreactor storage, and for storage requirements assuming maximum-at-reactor plus intra-utility transshipment of spent fuel. 


\section{ACKNOWLEDGMENTS}

The authors thank K. A. Ekblaw, and R. G. Rau for their assistance in performing the work described in this report. 



\section{CONTENTS}

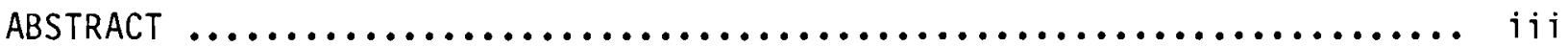

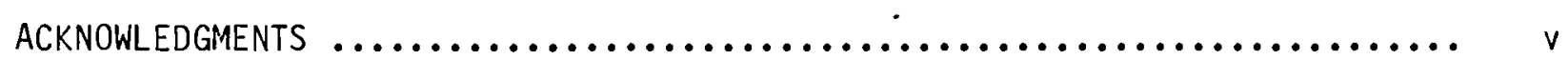

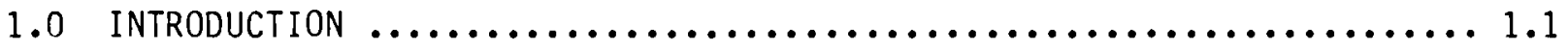

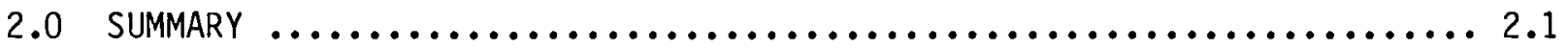

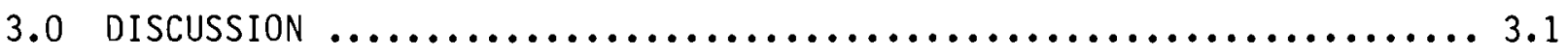

3.1 DATA BASE DESCRIPTION $\ldots \ldots \ldots \ldots \ldots \ldots \ldots \ldots \ldots \ldots \ldots \ldots \ldots . \ldots . \ldots . \ldots . \ldots$

3.2 DATA BASE ADJUSTMENT PROCEDURE $\ldots \ldots \ldots \ldots \ldots \ldots \ldots \ldots \ldots \ldots \ldots . . \ldots \ldots \ldots$

3.2.1 Step 1 - Shift Utility Data Base Startup and

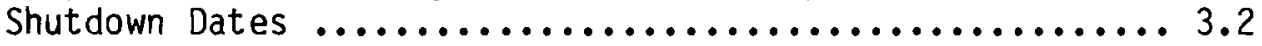

3.2.2 Step 2 - Calculate Electrical Energy Generation from the Utility SFDB $\ldots \ldots \ldots \ldots \ldots \ldots \ldots \ldots \ldots \ldots \ldots . . .3$

3.2.3 Step 3 - Adjustment of Utility Discharges to Match EIA Energy Projections ....................... 3.5

3.2.4 Step 4 - Adjustment of Burnups to Match EIA Discharge Projections ............................ 3.5

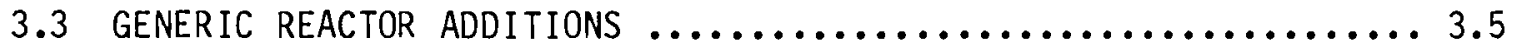

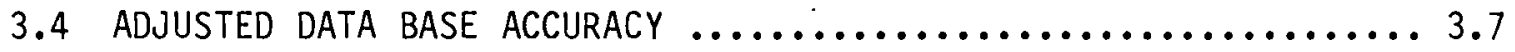

3.5 DATA BASE ADJUSTMENT RESULTS $\ldots \ldots \ldots \ldots \ldots \ldots \ldots \ldots \ldots \ldots \ldots \ldots \ldots \ldots \ldots \ldots \ldots$

3.6 SPENT FUEL STORAGE REQUIREMENTS $\ldots \ldots \ldots \ldots \ldots \ldots \ldots \ldots \ldots \ldots \ldots \ldots \ldots \ldots \ldots$

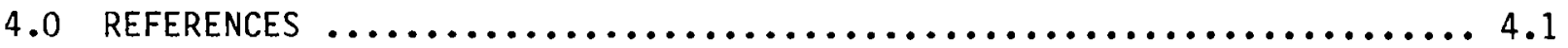

APPENDIX A - DETAILED DATA BASE RESULTS $\ldots \ldots \ldots \ldots \ldots \ldots \ldots \ldots \ldots \ldots \ldots \ldots \ldots \ldots \ldots \ldots$

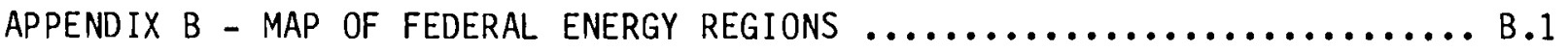




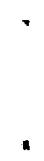

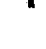




\section{FIGURES}

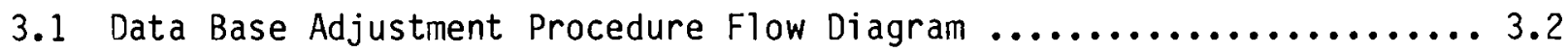

3.2 Installed EIA Nuclear Capacity Projections $\ldots \ldots \ldots \ldots \ldots \ldots \ldots \ldots \ldots . \ldots \ldots$

3.3 Projected EIA Nuclear Energy Generation $\ldots \ldots \ldots \ldots \ldots \ldots \ldots \ldots \ldots \ldots . \ldots \ldots$

3.4 Cumulative Spent Fuel Inventory for the Four Adjusted
Data Bases, and for the Unadjusted Utility Data ..................... 39

3.5 Cumulative Additional Storage Requirement--Maximum

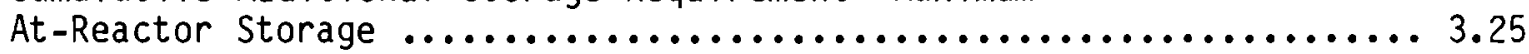

3.6 Cumulative Additional Storage Requirement--Maximum At-Reactor

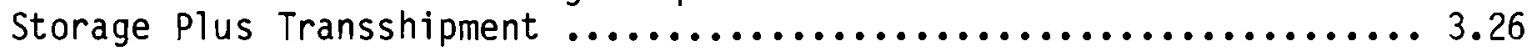

\section{TABLES}

3.1 Cycle to Batch Energy Ratio by Cycle .........................

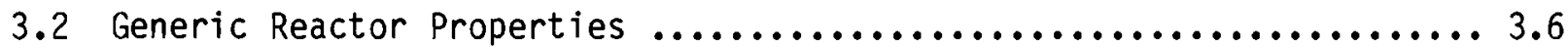

3.3 Federal Region Location $\ldots \ldots \ldots \ldots \ldots \ldots \ldots \ldots \ldots \ldots \ldots \ldots \ldots \ldots \ldots \ldots$

3.4 Allocation of Generic Reactors to Federal Region .................

3.5 No New Orders Case Capacity and Energy Comparison ............... 3.12

3.6 No New Orders Case Cumulative Discharges Compared to EIA

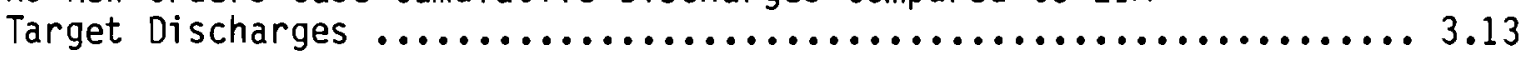

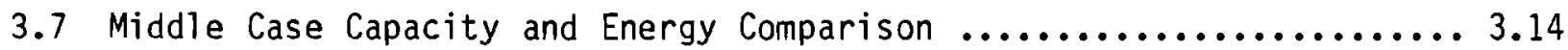

3.8 Middle Case Cumulative Discharges Compared to EIA .....................

3.9 Utility to EIA Required Energy Adjustment Factors--EIA/SFDB ....... 3.20

3.10 Cumulative Storage Requirements--Maximum AR Storage ...............21

3.11 Cumulative Storage Requirements--Maximum AR Storage Plus

Transshipment ........................................ 3.22 



\subsection{INTRODUCTION}

Under the provisions of the Nuclear Waste Policy Act of 1982 (NWPA), the Department of Energy (DOE) is responsible for the management and ultimate, permanent disposal of the civilian radioactive waste resulting from commercial nuclear power plant operations in the U.S. The Office of Civilian Radioactive Waste Management (OCRWM) has been established within the DOE to plan for and carry out this responsibility.

The greatest portion of the radioactive waste covered under this government responsibility will be spent nuclear fuel discharged from commercial nuclear power plants. Because most of the spent fuel that will ultimately require disposal has not yet been generated, planning for the management and disposal of this spent fuel must be largely based on projections of future discharges from commercial nuclear power plants.

The DOE Energy Information Administration (EIA) annually publishes projections of nuclear energy generation on an overall, industry composite basis (Mayes et a1. 1985). The EIA also publishes projections of spent-fuel discharges from commercial nuclear plants that are keyed to each nuclear energy generation projection (Moden et al. 1985). These EIA energy projections provide several scenarios that represent different assumptions about the future growth of nuclear energy capacity in the U.S. Multiple scenarios allow analysis of the sensitivity of results and decisions to varying assumptions.

The OCRWM plans for management and disposal of spent fuel are based on the EIA nuclear energy projections. However, the EIA projections do not contain the specific reactor-by-reactor information that is needed to perform detailed analyses of relevant issues such as waste system logistics, requirements for interim storage of spent fuel, or anticipated variations in the physical characteristics of the spent fuel to be received for disposal. Therefore, to provide a more detailed basis for such analyses, a study was performed in 1985 to develop detailed reactor-specific spent fuel discharge projections corresponding to the EIA nuclear energy growth projections (Heeb, Libby and Holter 1985). The present study is an update of the 1985 study. 
In the 1985 study, the basic source of data used to develop reactorspecific information was the Spent Fuel Data Base (SFDB) maintained by Pacific Northwest Laboratory (PNL) for the DOE Commercial jpent Fuel Management (CSFM) Program. The information in this data base is supplied directly by the nuclear utilities. The 1985 SFDB consists of detailed historical data (inclusive to 1984) and projected data on reactor operation, spent-fuel discharges and shipments, and spent-fuel storage and handling capabilities. The SFDB is based on utility information collected by EIA and supplied :0 PNL for the production of new detailed data bases that are adjusted to meet EIA projections of nuclear capacity, energy generation, and spent-fuel discha^ges.

The CSFM Program uses the adjusted data bases for a number of planning purposes, including the preparation of annual projections of future requirements for additional spent-fuel storage capacity (DOE 1985a). The data base also provides the basic information on spent fuel for inclusion in the DOE Integrated Data Base (IDB) maintained by Oak Ridge National Laboratory (DOE $1985 b)$.

Two 1985 EIA nuclear growth scenarios were chisen as the bases for developing the 1985 reactor-specific spent fuel discharje projections: the Middle Case and the No New Orders Case. The Middle Case was previously selected by the OCRWM as the base case for waste management planning purposes. The No New Orders Case was included to provide an additional data set that could be used to analyze the sensitivity of analytical results and management decisions to possible reductions in nuclear growth in the U.S.

The No New Orders Case includes only nuclear sower plants that are currently operating and a subset of the plants currently under construction. EIA assumes that some of the plants currently under construction will not be completed, and that others will be delayed beyond official utility startup estimates. The Middle Case includes additional power Jlants, not currently on order, coming on line starting in 2001 to provide sufficient power generation capacity to correspond to the overall nuclear energy production rates in the EIA projections. In addition, for plants now under construction, startup dates are earlier and the assumed number of canceled plants is lower than for the No New Orders Case. 
In making projections of the amount of spent fuel discharged, the EIA considers cases of extended and constant burnup. In the Middle Case, it is assumed that by 1993 the reactors will extend their current burnups by 30 percent. The discharge burnups are assumed to be constant beyond 1993. However, extended burnup is the EIA base case for 1985. A Middle Case with constant burnup was also considered. In this case, the burnup level was maintained at the 1979 to 1984 historical levels through 2020. Thus, at projected annual energy generations forecasted by the Middle Case, two spent-fuel discharge projections are made that correspond to the constant and extended burnup assumptions (Moden et a1. 1985, Table D.2). A similar set of assumptions for burnup are made for the energy generation forecasted in the No New Orders Case (Moden et al. 1985, Table D.2).

The 1984 spent fuel data base containing utility-supplied information was altered to produce four adjusted data bases. The aggregate annual energy production and spent-fuel discharge on these data bases agree with:

1. No New Orders Capacity and Energy, Extended Burnup Discharges (NNOE)

2. No New Orders Capacity and Energy, Constant Burnup Discharges (NNOC)

3. Middle Case Capacity and Energy, Extended Burnup Discharges (MCE)

4. Middle Case Capacity and Energy, Constant Burnup Discharges (MCC)

In order to meet the installed-capacity and energy-generation requirements beyond the year 2000 for the Middle Case, it was necessary to add generic power plants to the population of named reactors. Reactor types consistent with currently existing power plants were chosen to represent these generic power plants. This permitted the detailed information for the generic power plants to be obtained from the SFDB in the same manner as for the currently existing or planned reactors. Each of the generic reactors was assigned to a specific federal energy region. 


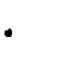




\subsection{SUMMARY}

The projected discharge burnups on the 1985 utility spent fuel data base, which contains historical information complete through 1984 plus projections from 1985, have been adjusted from utility-supplied values to produce agreement with the EIA nuclear energy generation and spent-fuel discharge forecasts.

Four data bases were produced that correspond to the following EIA cases:

1. No New Orders Capacity and Energy, Extended Burnup Discharges (NNOE)

2. No New Orders Capacity and Energy, Constant Burnup Discharges (NNOC)

3. Middle Case Capacity and Energy, Extended Burnup Discharges (MCE)

4. Middle Case Capacity and Energy, Constant Burnup Discharges (MCC)

The method developed to adjust the data allows the detail of the utility data base to remain intact, while the over-all nuclear generation and discharged amount are changed to match any uniform nuclear generation forecast. The adjustment procedure alters burnup to meet targets of annual energy production and amounts of spent fuel discharged.

The method used in developing the reactor-specific spent fuel discharge projections and the resulting data bases themselves are described in this report. Discussions of the method cover the following topics:

- a description of the data base

- data base adjustment procedures

- addition of generic power reactors

- accuracy of the data base adjustments.

The accumulated spent-fuel inventory for each of the four cases analyzed, and the original utility inventory projection are presented in Chapter 4.0.

Reactor-specific discharge and storage requirements for the Middle Case with extended burnup are presented in Appendix A. Table A.1 gives startup and shutdown dates for reactors in three scenarios: the Utilities Case, the Middle Case and the No New Orders Case. Table A.2 gives names and startup dates for the Middle Case generic reactors. Tables A.3 through A.8 show reactor-specific projections for the Middle Case with extended burnup. Tables A.3 and A.4 show the annual and cumulative discharges respectively. Tables A.5 through A.8 display the annual and cumulative storage requirements for the maximum 
at-reactor (AR) storage assumption, and for the maximum transshipment assumption. These compare directly to the storage requirements from the utilitysupplied data, which were reported by DOE (1985a).

The reactor-specific spent-fuel discharge projections contained in this report cover the period from 1985 through 2020 and include a total cumulative spent-fuel inventory of nearly 127,000 MTU for the Middle Case, Constant Burnup to 74,000 MTU for the No New Orders, Extended Burnup Case. A description of the method used to derive the data and the results is included in Chapter 3.0 . Detailed information that supports the discussions in the main body of the report, including descriptions of the capacity and fuel-discharge projections, are included in Appendix $A$. 


\subsection{DISCUSSION}

The 1985 utility spent fuel data base (Utility SFDB) is a compendium of information on U.S. commercial power reactor spent fuel and other reactorspecific information. It is based on data provided by the utility owners and represents their estimate of the amounts and characteristics of spent-fuel discharges. Electrical energy generation may be derived from the spent-fuel quantities and spent-fuel exposure contained in the data base.

\subsection{DATA BASE DESCRIPTION}

The SFDB contains a file for each reactor. The first portion of the file consists of time-independent information such as the location, various power ratings, dates of startup and final shutdown, and detailed information on fuel stored in the reactor's pool(s). The second portion consists of the historical record of fuel discharges by batch or sub-batch with discharge exposure range, number of assemblies, and the uranium mass for each batch. The second and succeeding portions are organized by operating cycle, that is, by the period between successive discharges. The third portion of the Utility SFDB contains projected discharge information for 1985 and beyond.

The electrical energy generation implicit in the Utility SFDB is entirely independent of the nuclear energy generation forecasts made by EIA. The Utility SFDB was modified to be consistent with these nuclear energy generation and discharged-fuel forecasts. The technical steps involved in adjusting the Utility SFDB to conform to the EIA projections are described in this section. The principal requirement of the adjustment was to retain as much of the detailed utility estimates of exposure, plant capacity factor, and discharge schedules as possible, while matching the EIA electrical energy generation and discharge forecasts.

\subsection{DATA BASE ADJUSTMENT PROCEDURE}

The steps in the adjustment procedure are shown in Figure 3.1. The adjustment process required construction of four major processors, each of which is described in the sections that follow. The adjustment process is 


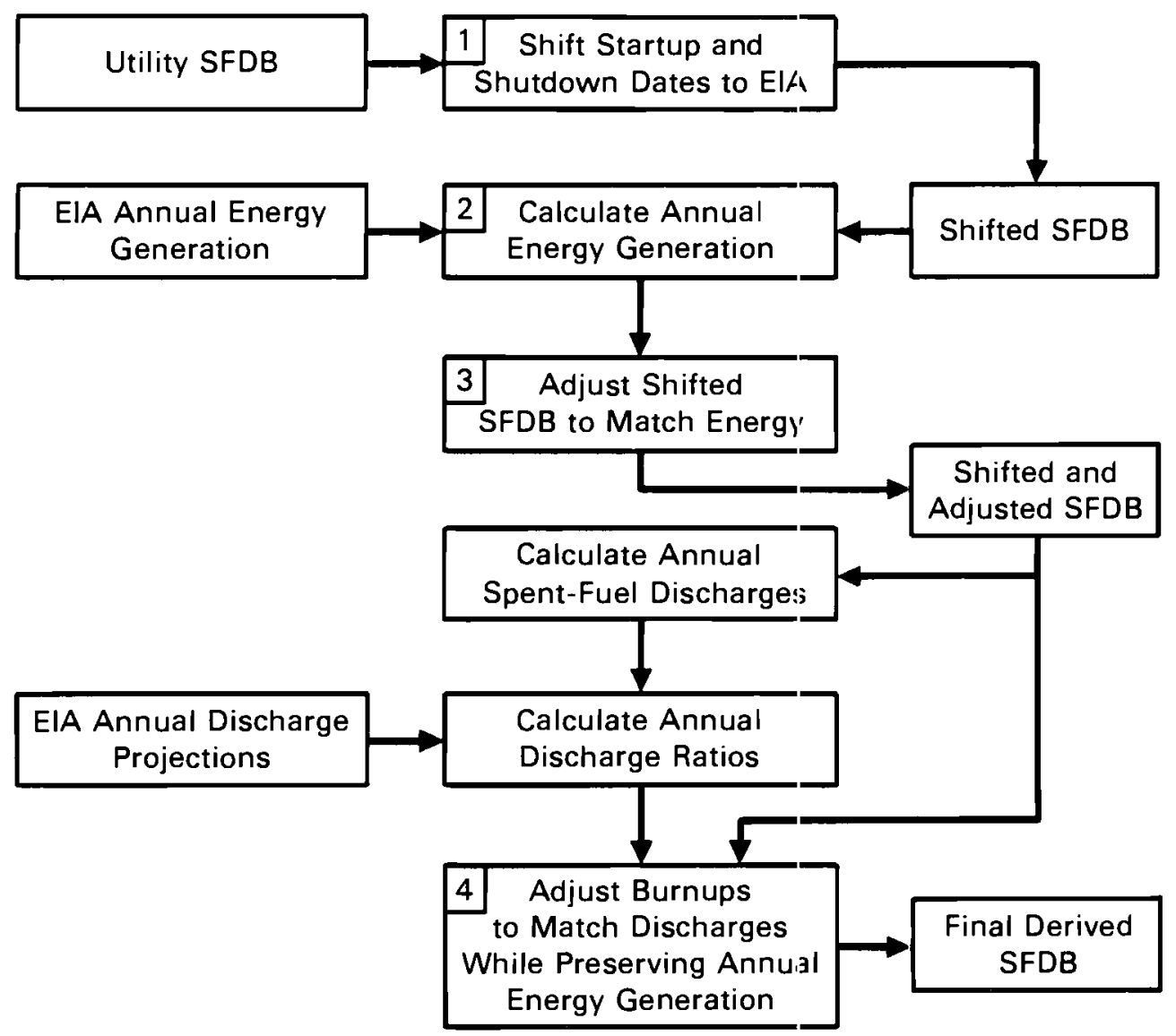

FIGURE 3.1. Data Base Adjustment Procedure Flow Diagram

entirely concerned with the time beyond 1984, since historical information is left unchanged by the adjustment process. The adjustment steps are numbered in the Figure 3.1 flow chart.

\subsubsection{Step 1 - Shift Utility Data Base Startup and Shutdown Dates}

EIA operable dates published by Mayes et al. (1985) for reactors starting up from 1984 to 2020 (pipeline reactors) were checked against the utilitysupplied start-up dates on the SFDB. No New Orders Case dates were shifted five months later than the EIA operable date. Middle Case startup dates were shifted six months prior to the EIA operable date. These different startup date adjustments were required to match the EIA installed nuclear capacity forecast. The start-up date for each reactor is shown in Appendix A, Table A.1. 
The utility-supplied pattern of discharges was displaced in time by the same adjustment time span used in shifting the startup dates. The shutdown dates were adjusted to agree with the EIA data. The full core was assumed to be discharged in the year following the last year of energy generation, in accordance with EIA ground rules. Middle Case generic reactors were added to match installed nuclear generation capacity. The installed capacity match was accomplished at the end of Step 1.

\subsubsection{Step 2 - Calculate Electrical Energy Generation from the Utility SFDB}

The cycle energy generation from the Utility SFDB was calculated by adding the product of discharge exposure and mass of the discharged batch for each batch discharged from the cycle. For reactors at equilibrium, the energy generated by the discharged fuel during its residence in the core is equal to the energy generated by the entire core during the discharge cycle. To illustrate, for a three-batch core-fuel-management plan at equilibrium, each in-core fuel batch generates a fixed fraction of core power during equilibrium operation: F1, F2, F3. If $E$ is the energy generation during each equilibrium cycle, the energy generated by the batch scheduled for discharge, ED, would be the sum of the energy generated by the discharge batch during each of the three cycles of residence:

$$
E D=E \times F 1+E \times F 2+E \times F 3
$$

By factoring out $E$, the equation is expressed as follows:

$$
E D=E \times(F 1+F 2+F 3)
$$

Since $F 1+F 2+F 3=1.0$ by definition, the discharge energy equals the energy generated during each equilibrium cycle:

$$
E D=E
$$

This conclusion would be true for any core equilibrium replacement fraction. For reactors just starting up, the ratio of first-cycle energy generation to 
first-discharge batch energy generation will not be unity, but will approximate the reciprocal of the core fraction discharged if power sharing by in-core batches is proportional to batch size. Thus for one-third core replacement, the ratio of first cycle energy to the energy generated by the fuel in the first discharge would approximate three. The second discharge ratio would approximate one half of this, or 1.5. The third discharge ratio would approximate unity and would remain unity for all subsequent discharges.

In actual practice power sharing between in-core fuel batches is not exactly equitable, and fuel management plans do not, specify that the same fraction of the core will be replaced for every refueling outage. However, when large numbers of reactors are involved and interest: is primarily in over-all energy generation over several years during which only a small fraction of the total energy generation is from plants not at equilibrium, average nonequilibrium ratios of cycle energy to batch energy may be approximated with sufficient accuracy. Detailed fuel management plans for several reactors were analyzed to obtain a more realistic ratio of cycle to batch energy. The ratios of cycle energy to batch energy were calculated and the results are summarized in the Table 3.1. The energy of a given cycle is calculated by adding the product of exposure and tonnage discharged over all batches from the cycle, and multiplying the resulting sum by the appropriate factor from Table 3.1.

The shifted SFDB energy is calculated for each plant in each cycle. The cycle energies are then allocated to each year according to the number of months in the year for that cycle. The annual energy generation projected by EIA is then compared to the annual sum of energy generation from the shifted SFDB. An annual correction factor equal to the EIA energy generation divided by the SFDB energy is then calculated. The target annual energies are then used in the final adjustment process.

TABLE 3.1. Cycle to Batch Energy Fiatio by Cycle

\begin{tabular}{|c|c|c|c|c|c|}
\hline & First & Second & Third & Fourth & Equilibrium \\
\hline PWR & 2.857 & 1.266 & 1.095 & 1.1773 & 1.000 \\
\hline$B W R$ & 3.663 & 1.404 & 1.111 & 1.058 & 1.000 \\
\hline
\end{tabular}




\subsubsection{Step 3 -Adjustment of Utility Discharges to Match EIA Energy}

Projections

The projected SFDB discharge amounts are modified to be consistent with the target energies. The adjustment assumptions were that no discharge batches of less than 11 assemblies would be modified, and that a slight adjustment to the utility-supplied exposure would be made to preserve an integral number of discharge assemblies in the batches of 11 or more assemblies. Adjusting only batches greater than ten assemblies assures that no exposure will be adjusted by more than ten percent in order to preserve an integral number of assemblies. The larger batch exposures are adjusted a little more than originally called for in order to compensate for the unadjusted fuel batches having ten or less assemblies. The original adjustment ratio of target-to-data base generated energy is preserved by this process.

\subsubsection{Step 4 - Adjustment of Burnups to Match EIA Discharge Projections}

In Step 4, the amount of fuel discharged is changed to match the EIA projected discharges reported by Moden et al. (1985). To preserve annual energy generation, the burnups are adjusted so that the energy (product of burnup and discharged weight) remains constant. The original utility data base contains a wide variation in discharge burnup. Those utilities whose data contained planned increases in burnup would be forced into discharge burnups far beyond the range of technical feasibility by a single global adjustment ratio applied each year. To avoid this result, a ceiling on PWR and BWR burnup is provided. No adjustments are made beyond the ceiling once it is reached. When the ceiling is encountered on one or more plants, the adjustment in burnup for the plants below the ceiling is increased so that the required annual adjustment factor is realized each year.

\subsection{GENERIC REACTOR ADDITIONS}

The SFDB contains information only on those reactors that were operating, or were in the planning stage by some U.S. utility in 1984. In order to meet EIA energy generation forecast after 1984 for the Middle Case, it is necessary to include generic reactors in the shifted data base at Step 1 in the adjustment process (see previous discussion and Figure 3.1). Two actual reactors 
were selected to represent the generic PWR and BWR. Both were nominal 1100 MWe plants and both were on an 18 month refueling schedule. The PWR design exposure was 38,077 MWd/MTIHM, and the BWR burnup was 32,670 MWd/MTIHM. Relevant details for the two generic plant types are shown in Table 3.2.

Increases in capacity do not in general represent the addition of an integral number of plants, each with a fixed plant capacity. Fractional additions to capacity are represented by delayed startup of one of the plants of each plant type that are otherwise started up in January each year to maintain the correct cumulative capacity. Thus if the accumulated new capacity is equivalent to 15.65 generic plants by a given year, one of the plants will be started up in the fourth month of that year: $(1.0-0.65) \times 12.0=4.2$. The start-up of integral additions to capacity is done in accordance with the EIA convention of commercial operation additions in July of each year with a six month interval between first electrical generation and commercial operation.

It is important for logistics modeling to maintain reasonable geographic accuracy in the projected spent fuel discharges. Maintenance of geographic accuracy requires that the generic reactors be added with site diversity. The first step in providing site diversity is to assign each generic reactor to a federal energy region. The location of each federal energy region is shown in Table 3.3, and a map of the regions in reproduced in Appendix $B$.

The number of reactors added to each region is determined by apportioning the plants according to the projected electrical capacity in 1995 from nuclear power plants in the region. The projected capacities are taken from the No New Orders Case projections for 1995. It is assumed that a similar

\section{TABLE 3.2. Generic Reactor Properties}

\begin{tabular}{lcc} 
& \multicolumn{1}{c}{ PWR } & BWR \\
\cline { 2 - 3 } Rated Power Level & $1,100 \mathrm{MWe}$ & $1,100 \mathrm{MWe}$ \\
Thermal Power Level & $3,350 \mathrm{MWth}$ & $3,400 \mathrm{MWth}$ \\
Fuel Cycle Length & $18 \mathrm{Months}$ & $18 \mathrm{Months}$ \\
Equilibrium Enrichment & $3.65 \mathrm{wt} \%$ & $3.0 \mathrm{wt} \%$ \\
Discharge Exposure & $38,077 \mathrm{MWd} / \mathrm{MT}^{-}$ & $32,670 \mathrm{MWd} / \mathrm{MT}$
\end{tabular}


TABLE 3.3. Federal Region Location

\begin{tabular}{rll}
$\begin{array}{c}\text { Federal } \\
\text { Energy Region }\end{array}$ & & \multicolumn{1}{c}{ Location } \\
\cline { 1 - 1 } I & & New England \\
III & & New York/New Jersey \\
IV & & South Atlantic \\
V & & Midwest \\
VI & Southwest \\
VII & Central \\
VIII & North Central \\
IX & West \\
$X$ & Northwest
\end{tabular}

regional proportion of installed nuclear capacity applies beyond 1995 . Because of specific regional considerations, some exceptions are made with this method for assigning generic reactors to sites. For example, the number of available sites within Region III is 19 , so one of the 20 allocated reactors is relocated to the adjacent Region IV. Table 3.4 shows the 1995 installed capacities, the number of allocated generic reactors that would been in each region based on the No New Orders Case 1995 capacity, and the actual number of generic plants in each region. Table A.2 in Appendix A shows the actual federal energy region placement and date of commercial operation for each generic reactor.

The siting of generic plants within a given federal region was accomplished using the method described by Holter et al. (1986). The method develops a numerical rating for each postulated generic site in each of the 10 federal energy regions. The numerical rating defines the sequence in which each potential site is chosen. The generic reactors are then assigned chronologically in order of their startup date using this priority sequence.

\subsection{ADJUSTED DATA BASE ACCURACY}

The adjustment procedure is not a numerically exact process. Approximations are required during the assignment of annual energies to operating cycle 
TABLE 3.4. Allocation of Generic Reactors to Federal Region

\begin{tabular}{|c|c|c|c|c|}
\hline $\begin{array}{l}\text { Federal } \\
\text { Energy } \\
\text { Region } \\
\end{array}$ & $\begin{array}{c}\text { Installed } \\
\text { Capacity (MWe) }\end{array}$ & $\begin{array}{l}\text { Percent } \\
\text { of Total }\end{array}$ & $\begin{array}{l}\text { A1 loc:ated } \\
\text { Reactiors }\end{array}$ & $\begin{array}{c}\text { Middle Case } \\
\text { Generic Reactors }\end{array}$ \\
\hline I & 6,637 & 6.2 & 10 & 10 \\
\hline I I & 8,767 & 8.2 & 14 & 14 \\
\hline I I I & 12,889 & 12.0 & 20 & 19 \\
\hline IV & 32,337 & 30.2 & $5:$ & 53 \\
\hline V & 22,668 & 21.2 & 35 & 35 \\
\hline VI & 7,399 & 6.9 & 12 & 11 \\
\hline VII & 3,345 & 3.1 & 5 & 7 \\
\hline VI I I & 330 & 0.3 & $i$ & 1 \\
\hline IX & 10,467 & 9.8 & 16 & 16 \\
\hline$x$ & 2,230 & 2.1 & 3 & 1 \\
\hline
\end{tabular}

energies. A linear weighting scheme was used which assigns the annual energy to an operating cycle in proportion to the number of months of that year that are in the cycle.

Using the following array and index definitions the adjustment procedure approximation can be expressed in general terms:

$$
\begin{aligned}
& I=\text { cycle index, } J \text { = year index, } R=\text { reactor index } \\
& M(I, J, R)=\text { months in cycle } I \text {, year } J \text {, reactor } R \\
& E D(I, R)=\text { shifted data base energy for cycle }[\text {, reactor } R \\
& E A(J)=\text { annual target energy generation, year } J \text {. }
\end{aligned}
$$

The annual shifted data base energy for reactor $R$ is calculated using the equation:

$$
\operatorname{JED}(\mathrm{J}, \mathrm{R})=\sum_{\mathrm{I}} M(\mathrm{I}, \mathrm{J}, \mathrm{R}) / 12 \times E D(I, R)
$$

The annual energies are then summed over reactors:

$$
\operatorname{SED}(J)=\sum_{R} \operatorname{JED}(J, R)
$$


A ratio to the annual target energies is calculated:

$$
\operatorname{RATIO}(\mathrm{J})=\operatorname{EA}(\mathrm{J}) / \operatorname{SED}(\mathrm{J})
$$

An effective ratio is calculated for each reactor cycle using $C L(I, R)$, the cycle length in months for cycle I, reactor $\mathrm{R}$ :

$$
\operatorname{ERATIO}(I, R)=\int_{J} M(I, J, R) / C L(I, R) \times \operatorname{RATIO}(J)
$$

The effective ratio is used to produce an adjusted data base energy for cycle $I$, reactor $R$ and the discharged amounts are changed by the same ratio:

$$
\operatorname{ADJED}(I, R)=\operatorname{ERATIO}(I, R) \times E D(I, R)
$$

This process produces an adjusted data base. To test the accuracy of the adjusted data base, the adjusted cycle energies are reallocated to years, added up over all the reactors operating in a given year and compared with the target energy $E A(J)$ in test year $J$. In other words, the following hypothetical equality is tested for each year $\mathrm{J}$ :

$$
\begin{aligned}
& \sum_{R} \operatorname{YADJED}(J, R) \stackrel{?}{=} \operatorname{EA}(\mathrm{J}), \text { where } \\
& \operatorname{YADJED}(J, R)=\sum_{I} M(I, J, R) / 12 \times \operatorname{ADJED}(I, R)
\end{aligned}
$$

To determine the mathematical conditions under which Equation 3.9 is valid, $\operatorname{YADJED}(J, R)$ can be analyzed by substituting the array definitions into the preceding equation which defines $\operatorname{YADJED}(J, R)$ :

$$
\frac{\sum_{R} \sum_{I} M(I, J, R) / 12 \times E D(I, R) \times \sum_{J} M(I, J, R) / C L(I, R) \times E A(J)}{\sum_{R} \sum M(I, J) / 12 \times E D(I, R)}
$$


The double summation over $R$ and $I$ in the numerator is identical to the double sum in the denominator.

When $\sum_{j} M(I, J, R) / C L(I, R) \times E A(J)$ reduces to $E A(J)$ the adjustment is exact.

The array $\mathrm{CL}(I, R)$ is the cycle length in montis. If $M(I, J, R)$ is summed over $J$ years for a given reactor cycle it must equal the cycle length:

$$
C L(I, R)=\sum_{J} M(I, J, R)
$$

The sum can be reduced to unity for each value of $I$ and $R$ only if all of the annual target energies in the sum $E A(J)$ are equal and can be factored out of the sum. When this assumption is made the fraction reduces to $E A(J)$ and the adjustment method is exact. Thus the adjusted SFDB annual energy will equal the target EIA energy exactly only if those energies are equal for a period of time before and after the test year. Forecasts wherein the annual energy generation changes in abrupt steps will be less accurately adjusted. Calculation of the accuracy of each adjusted data base is, therefore, a mandatory step in the adjustment process.

The data base adjustment process allows a complete check on the accuracy of the approximation. When the final adjusted data base is produced, the annual adjusted energies can be calculated and sumned over all reactors by performing Step 2. The resulting annual sum can be compared to the target annual energies by performing Step 3. The resulting annual energy adjustment ratios are generally very close to unity. Thus the adjustment process converges to within a few percent after a single iteration.

The adjustment process approximation can be explained by a numerical example: If an 18-month cycle were made up of three months for the first year, 12 months for the next year, and three months for the third year, the cycle energy would be: 


$$
\text { ECYCLE }=3 / 12 \times E 1+12 / 12 \times E 2+3 / 12 \times E 3
$$

where E1, E2, and E3 are the annual energies of the first, second and third years respectively. If the annual energies are the target EIA energies, then ECYCLE would be the cycle energy generation on the database that would exactly match the target energies. If ESFDB is the unadjusted cycle energy, the adjustment ratio will be ECYCLE/ESFDB. After the adjustment is made, ESFDB is replaced by ECYCLE, and all discharges are adjusted by the adjustment factor ECYCLE/ESFDB. The allocation of cycle energy to years would be EADJ1 $=3 / 18 \mathrm{x}$ ECYCLE for the first year. The original allocation is $3 / 12 \times E 1$. If the adjustment is exact, the ratio of adjusted first year allocation, EADJ1, to $3 / 12 \times E 1$ should equal unity. The ratio is:

$$
\frac{3 / 18 \times(3 / 12 \times E 1+12 / 12 \times E 2+3 / 12 \times E 3)}{3 / 12 \times E 1}
$$

This expression will be unity only if $E 1=E 2=E 3$.

\subsection{DATA BASE ADJUSTMENT RESULTS}

The results of adjustments to the SFDB are highlighted in this section. Specifically the comparison between the target EIA capacities and energy generation, and the after-adjustment capacities and energy generation is shown. It is impossible to achieve numerical identity for two basic reasons. First, the data sources are different. The utilities supply the plant capacity numbers in the SFDB. EIA sources are independent, and while there may be differences in some detail, the over-all agreement should be quite close. Second, the energygeneration adjustment algorithm is not exact where adjacent year target energies differ greatly. This generally produces some minor differences between the target energy and the energy used to adjust spent fuel amounts.

Table 3.5 shows the installed capacity and energy generated by the target EIA forecast, and the SFDB No New Orders Case. The capacities of the adjusted data base are within 10 percent of the EIA values for the years 1986 through 
TABLE 3.5. No New Orders Case Capacity and Energy Comparison

\begin{tabular}{|c|c|c|c|c|}
\hline \multirow[b]{2}{*}{ Year } & \multicolumn{2}{|c|}{$\begin{array}{l}\text { Installed } \\
\text { Capacity } \\
\text { (Gigawatts) }\end{array}$} & \multicolumn{2}{|c|}{$\begin{array}{c}\text { Electrical } \\
\text { Energy } \\
\text { (Terawatt- } \\
\text { hours) }\end{array}$} \\
\hline & EIA & SFDB & $E I A$ & SFDB \\
\hline 1985 & 77.0 & 76.4 & $360.1)$ & 358.2 \\
\hline 1986 & 82.6 & 86.7 & 397.3 & 395.3 \\
\hline 1987 & 88.2 & 97.4 & 435.15 & 440.9 \\
\hline 1988 & 93.8 & 102.3 & 473.4 & 475.0 \\
\hline 1989 & 99.4 & 103.5 & $511 . ?$ & 517.5 \\
\hline 1990 & 105.0 & 104.7 & $549.1)$ & 544.7 \\
\hline 1991 & 105.6 & 105.8 & 557.3 & 558.4 \\
\hline 1992 & 106.2 & 107.1 & 566.15 & 567.7 \\
\hline 1993 & 106.8 & 107.1 & 575.4 & 577.0 \\
\hline 1994 & 107.4 & 107.1 & $584 . ?$ & 5 \\
\hline 1995 & 108.0 & 107.1 & $593.1)$ & \\
\hline 1996 & 107.6 & 107.1 & 595.5 & \\
\hline 1997 & 107.2 & 107.1 & 598.? & \\
\hline 1998 & 106.8 & 106.9 & 600.8 & \\
\hline 1999 & 106.4 & 106.9 & 603.4 & 60 \\
\hline 2000 & 106.0 & 106.5 & $606.1)$ & \\
\hline 2001 & 106.0 & 106.5 & $606.1)$ & \\
\hline 2002 & 106.0 & 106.4 & $606.1)$ & \\
\hline 2003 & 106.0 & 106.4 & $606.1)$ & \\
\hline 2004 & 106.0 & 106.3 & 606.13 & \\
\hline 2005 & 106.0 & 105.6 & $606.1]$ & \\
\hline 2006 & 105.6 & 105.6 & $603 . ?$ & \\
\hline 2007 & 105.2 & 104.4 & 600.4 & \\
\hline 2008 & 104.8 & 104.1 & 597.15 & \\
\hline 2009 & 104.4 & 104.1 & 594.3 & \\
\hline 2010 & 104.0 & 104.1 & 592.1 & \\
\hline 2011 & 95.0 & 103.4 & 541.5 & \\
\hline 2012 & 86.0 & 91.3 & 491.? & \\
\hline 2013 & 77.0 & 79.6 & 440.3 & \\
\hline 2014 & 68.0 & 68.1 & 390.4 & \\
\hline 2015 & 59.0 & 59.0 & $340.1]$ & \\
\hline 2016 & 56.4 & 57.8 & 324.13 & \\
\hline 2017 & 53.8 & 56 & 309.5 & \\
\hline 201 & 51.2 & 53.0 & 294.4 & \\
\hline 2015 & 48.6 & 48.4 & 279.2 & \\
\hline$\angle U<U$ & 46.0 & 46.1 & $264.1)$ & \\
\hline
\end{tabular}

1988 and are much closer during the years when generation capacity is stable. The annual electrical energy generation is generally within 1 percent of the EIA target value except in the year 2011 where the SFDB is high by 4 percent. 
Table 3.6 shows the cumulative discharges for the Constant Burnup and Extended Burnup cases compared to the EIA target cumulative discharges described by Moden et a1. (1985). Cumulative spent fuel discharges from the SFDB are generally within 1 percent of the EIA forecast value.

TABLE 3.6. No New Orders Case Cumulative Discharges Compared to EIA Target Discharges.

\begin{tabular}{|c|c|c|c|c|}
\hline Year & $\begin{array}{c}\text { Constant } \\
\text { Discharges }\end{array}$ & $\begin{array}{l}\begin{array}{l}\text { Burnup } \\
\text { (MTIHM) }\end{array} \\
\text { SFDB }\end{array}$ & $\begin{array}{r}\text { Extended } \\
\text { Discharge } \\
\text { EIA }\end{array}$ & $\begin{array}{l}\begin{array}{l}\text { Burnup } \\
\text { (MTIHM) }\end{array} \\
\text { SFDB }\end{array}$ \\
\hline 1984 & 11,442 & 11,424 & 11,442 & 11,424 \\
\hline 1985 & 12,679 & 12,801 & 12,474 & 12,759 \\
\hline 1986 & 14,178 & 14,205 & 14,040 & 14,054 \\
\hline 1987 & 15,659 & 15,829 & 15,330 & 15,655 \\
\hline 1988 & 17,554 & 17,656 & 17,277 & 17,328 \\
\hline 1989 & 19,702 & 19,646 & 19,071 & 19,112 \\
\hline 1990 & 21,643 & 21,711 & 20,743 & 20,995 \\
\hline 1991 & 23,754 & 23,772 & 22,936 & 22,762 \\
\hline 1992 & 25,896 & 25,953 & 24,370 & 24,757 \\
\hline 1993 & 28,193 & 28,193 & 26,738 & 26,494 \\
\hline 1994 & 30,480 & 30,403 & 28,147 & 28,564 \\
\hline 1995 & 32,538 & 32,623 & 30,591 & 30,357 \\
\hline 1996 & 34,864 & 34,928 & 32,125 & 32,401 \\
\hline 1997 & 37,395 & 37,219 & 34,289 & 34,213 \\
\hline 1998 & 39,417 & 39,516 & 36,031 & 36,057 \\
\hline 1999 & 41,756 & 41,831 & 37,666 & 37,909 \\
\hline 2000 & 44,345 & 44,168 & 39,861 & 39,635 \\
\hline 2001 & 46,435 & 46,485 & 41,209 & 41,627 \\
\hline 2002 & 48,716 & 48,822 & 43,635 & 43,370 \\
\hline 2003 & 51,366 & 51,186 & 45,100 & 45,316 \\
\hline 2004 & 53,535 & 53,515 & 47,061 & 47,049 \\
\hline 2005 & 55,710 & 55,853 & 48,835 & 48,825 \\
\hline 2006 & 385 & 58,163 & 50,437 & 50,708 \\
\hline 2007 & 465 & 60,504 & 52,719 & 52,474 \\
\hline 2008 & 62,739 & 826 & 142 & 54,498 \\
\hline 2009 & 65,359 & 65,220 & 517 & 56,287 \\
\hline 2010 & 67,678 & 67,753 & 58,094 & 58,345 \\
\hline 2011 & 70,378 & 70,465 & 60,318 & 60,550 \\
\hline 2012 & 499 & 73,496 & 63,135 & 63,129 \\
\hline 2013 & .774 & 76,312 & 65,853 & 65,646 \\
\hline 2014 & 78,825 & 78,775 & 67,877 & 67,692 \\
\hline 2015 & 80,919 & 80,570 & 69,280 & 69,350 \\
\hline 2016 & 70 & 82,237 & 70,740 & 70,637 \\
\hline 2017 & 333 & 83,655 & 71,739 & 71,845 \\
\hline 2018 & & 84,997 & 72,914 & 72,866 \\
\hline 2019 & 96 & 86,196 & 73,809 & 73,871 \\
\hline 2020 & 87,449 & 87,283 & 74,635 & 74,778 \\
\hline
\end{tabular}


Annual capacity and energy generation comparisons for the Middle Case are shown in Table 3.7. As in the No New Orders Case the agreement in installed capacity between the SFDB and EIA is within 10 percent in the years 1986

TABLE 3.7. Middle Case Capacity and Energy Comparison

\begin{tabular}{|c|c|c|c|c|}
\hline \multirow[b]{2}{*}{ Year } & \multicolumn{2}{|c|}{$\begin{array}{l}\text { Installed } \\
\text { Capacity } \\
\text { (Gigawatts) }\end{array}$} & \multicolumn{2}{|c|}{$\begin{array}{l}\text { Electrical Energy } \\
\text { (Terawatt-hours) }\end{array}$} \\
\hline & $E I A$ & SFDB & EIA & SFDB \\
\hline 1985 & 80.5 & 80.3 & 372.0 & 374.2 \\
\hline 1986 & 86.3 & 92.6 & 412.4 & 416.5 \\
\hline 1987 & 92.1 & 100.4 & 452.8 & 454.9 \\
\hline 1988 & 97.9 & 104.3 & 493.2 & 498.2 \\
\hline 1989 & 103.7 & 106.1 & 533.6 & 530.5 \\
\hline 1990 & 109.6 & 109.3 & 574.0 & 568.7 \\
\hline 1991 & 111.0 & 112.9 & 588.0 & 586.3 \\
\hline 1992 & 112.4 & 115.3 & 602.0 & 620.5 \\
\hline 1993 & 113.9 & 116.4 & 616.0 & 619.3 \\
\hline 1994 & 115.3 & 116.4 & 630.0 & 629.8 \\
\hline 1995 & 116.8 & 116.4 & 644.0 & 641.5 \\
\hline 1996 & 116.5 & 116.4 & 647.0 & 647.2 \\
\hline 1997 & 116.3 & 116.3 & 650.0 & 649.7 \\
\hline 1998 & 116.1 & 116.2 & 653.0 & 653.2 \\
\hline 1999 & 115.9 & 115.8 & 656.0 & 656.6 \\
\hline 2000 & 115.7 & 115.7 & 659.0 & 658.8 \\
\hline 2001 & 122.3 & 120.8 & 690.2 & 685.0 \\
\hline 2002 & 129.0 & 128.6 & 721.4 & 722.4 \\
\hline 2003 & 135.6 & 135.2 & 752.6 & 752.2 \\
\hline 2004 & 142.3 & 141.6 & 783.3 & 783.2 \\
\hline 2005 & 149.0 & 148.4 & 815.0 & 816.2 \\
\hline 2006 & 155.6 & 153.8 & 852.2 & 858.7 \\
\hline 2007 & 162.2 & 158.3 & 889.4 & 881.2 \\
\hline 2008 & 168.8 & 164.5 & 926.5 & .4 \\
\hline 2009 & & & 963.3 & .07 \\
\hline 2010 & 182.0 & 182.0 & 1001.3 & 988.6 \\
\hline 2011 & 188.8 & 194.0 & 1031.6 & 1031.1 \\
\hline 2012 & 195.6 & 203.2 & 1062.2 & 1062.2 \\
\hline 2013 & 202.4 & 209.9 & 1092.3 & 1097.8 \\
\hline 2014 & 209.2 & 211.9 & 1123.4 & 1117.6 \\
\hline 2015 & 216.0 & 216.4 & $1154 . J$ & 1144.3 \\
\hline 2016 & 222.4 & 225.0 & 1197.2 & 1198.9 \\
\hline 2017 & 228.8 & 226.8 & 1240.7 & 1228.4 \\
\hline 2018 & 235.2 & 235.3 & 1283.5 & 1271.0 \\
\hline 2019 & 241.6 & 242.5 & 1326.3 & 1315.4 \\
\hline 2020 & 248.0 & 248.4 & 1370.10 & 1352.7 \\
\hline
\end{tabular}


through 1989, and within one percent in the stable middle years except for 2011 and 2012 when disagreement is larger than 1 percent but less than 10 percent. The annual electrical generation is within 1 percent except for the years 2010 and 2011.

The Middle Case cumulative discharges are compared to EIA target discharges in Table 3.8. The agreement is consistently within 1 percent.

Annual reactor-by-reactor discharge projections for the Middle Case with extended burnup are shown in Table A.3 of Appendix A. Table A.4 shows the cumulative inventory at each reactor for the same Middle Case.

Figure 3.2 illustrates the installed capacities of the Middle Case and the No New Orders Case according to the EIA (Mayes et al. 1985). Figure 3.3 shows a similar comparison of annual energy generation for the two EIA forecasts. The cumulative spent fuel discharges are shown in Figure 3.4.

The required adjustment factors to obtain an agreement between the utility and the EIA forecasted annual energy generation are shown in Table 3.9. In general, the utility-supplied energy generation had to be reduced by approximately 5 percent to align with the EIA forecasted No New Orders Case generation. Less than 5 percent adjustment was required in the Middle Case. The information supplied by the utilities was more optimistic than EIA estimates of the annual energy generation.

\subsection{SPENT FUEL STORAGE REQUIREMENTS}

The final result to be reported is the analysis of additional fuel storage requirements. The two reference cases covered in this analys is are based on the maximum at-reactor storage capacities of the individual reactor pools, as determined by the utilities. Both cases include allowances for maintaining full core discharge capability, also referred to as full core reserve (FCR), for each separate reactor. A single FCR is assumed to be maintained for all units at multiple reactor stations employing either a single common spent-fuel storage pool, or separate pools with interconnections allowing transfer of spent fuel around them. The only difference between the two references cases 
TABLE 3.8. Middle Case Cumulative Discharges Compared to EIA

\begin{tabular}{|c|c|c|c|c|}
\hline & $\begin{array}{r}\text { Constant } \\
\text { Discharges }\end{array}$ & $\begin{array}{l}\text { urnup } \\
\text { (MTIHM) }\end{array}$ & $\begin{array}{l}\text { Extended } \\
\text { Discharge }\end{array}$ & $\begin{array}{l}\begin{array}{l}\text { Burnup } \\
\text { (MTIHM) }\end{array} \\
\text { SFDB }\end{array}$ \\
\hline Year & $E I A$ & SFDB & $E A$ & SFDB \\
\hline 1984 & 11,442 & 11,424 & 11,442 & 11,423 \\
\hline 1985 & 12,679 & 12,659 & 12,474 & 12,436 \\
\hline 1986 & 14,178 & 14,155 & 14,040 & 13,979 \\
\hline 1987 & 15,754 & 15,728 & 15,426 & 15,340 \\
\hline 1988 & 17,680 & 17,648 & 17,498 & 17,384 \\
\hline 1989 & 19,757 & 19,725 & 19,287 & 19,146 \\
\hline 1990 & 21,792 & 21,757 & 20,950 & 20,781 \\
\hline 1991 & 24,085 & 24,050 & 23,149 & 22,956 \\
\hline 1992 & 26,275 & 26,237 & 24,693 & 24,537 \\
\hline 1993 & 28,635 & 28,593 & 27,193 & 27,015 \\
\hline 1994 & 31,010 & 30,860 & 28,730 & 28,483 \\
\hline 1995 & 33,530 & 33,378 & 31,441 & 31,169 \\
\hline 1996 & 35,919 & 35,782 & 32,946 & 32,736 \\
\hline 1997 & 38,571 & 38,427 & 35,436 & 35,178 \\
\hline 1998 & 41,048 & 40,902 & 37,445 & 37,185 \\
\hline 1999 & 43,507 & 43,372 & 39,137 & 38,876 \\
\hline 2000 & 46,118 & 45,978 & 41,658 & 41,363 \\
\hline 2001 & 48,569 & 48,427 & 42,989 & 42,989 \\
\hline 2002 & 51,284 & 51,143 & 45,723 & 45,697 \\
\hline 2003 & 54,047 & 53,901 & 47,302 & 47,367 \\
\hline 2004 & 56,783 & 56,633 & 50,029 & 50,061 \\
\hline 2005 & 59,729 & 59,584 & 52,446 & 52,453 \\
\hline 2006 & 63,246 & 63,081 & 54,548 & 54,527 \\
\hline 2007 & 66,290 & 66,188 & 57,863 & 57,830 \\
\hline 2008 & 69,580 & 69,470 & 60,048 & 60,236 \\
\hline 2009 & 73,645 & 73,522 & 63,580 & 63,738 \\
\hline 2010 & 77,417 & 77,279 & 66,381 & 66,784 \\
\hline 2011 & 81,605 & 81,462 & 69,914 & 295 \\
\hline 2012 & 86,479 & 86,302 & 74,259 & 584 \\
\hline 2013 & 92,182 & 91,982 & 78,539 & 78,81 \\
\hline 2014 & 96,668 & 96,444 & 82,731 & 82,95 \\
\hline 2015 & 101,187 & 100,950 & 86,416 & 628 \\
\hline 201 & 106,129 & 105,860 & 90,502 & 90,674 \\
\hline 2017 & 111,472 & 111,180 & 535 & 656 \\
\hline 2018 & 116,102 & 115,780 & 620 & 699 \\
\hline 2019 & 121,395 & 121,040 & 102,684 & 102,199 \\
\hline 2020 & 126 & 126,250 & 106,404 & 444 \\
\hline
\end{tabular}

is in the consideration of transshipment to other reactor sites. The first reference case assumes that transshipments occur only as currently planned by the utilities. The second reference case includes the additional assumption 


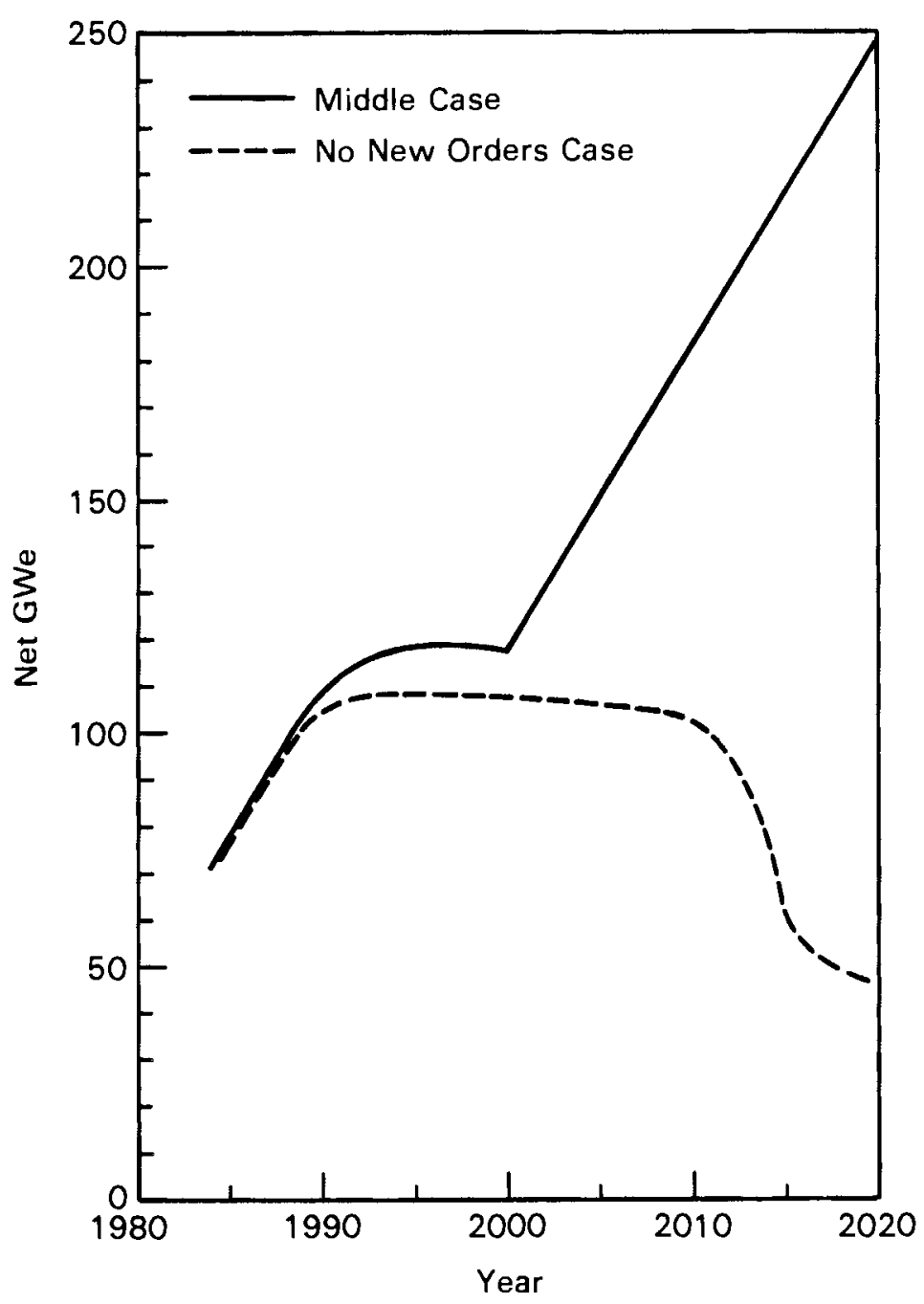

FIGURE 3.2. Installed EIA Nuclear Capacity Projections

that there are no constraints on transshipments of spent fuel among reactors of like type (i.e. among BWRs or among PWRs within a given utility system). This assumption allows unused spent-fuel storage capacity at one reactor to offset needs for additional capacity at another reactor in the same utility system, thus delaying the utility's need for additional storage capacity. Such transshipments are included in the spent-fuel management plans of several utilities. The two reference cases therefore define a range of potential storage requi rements. 


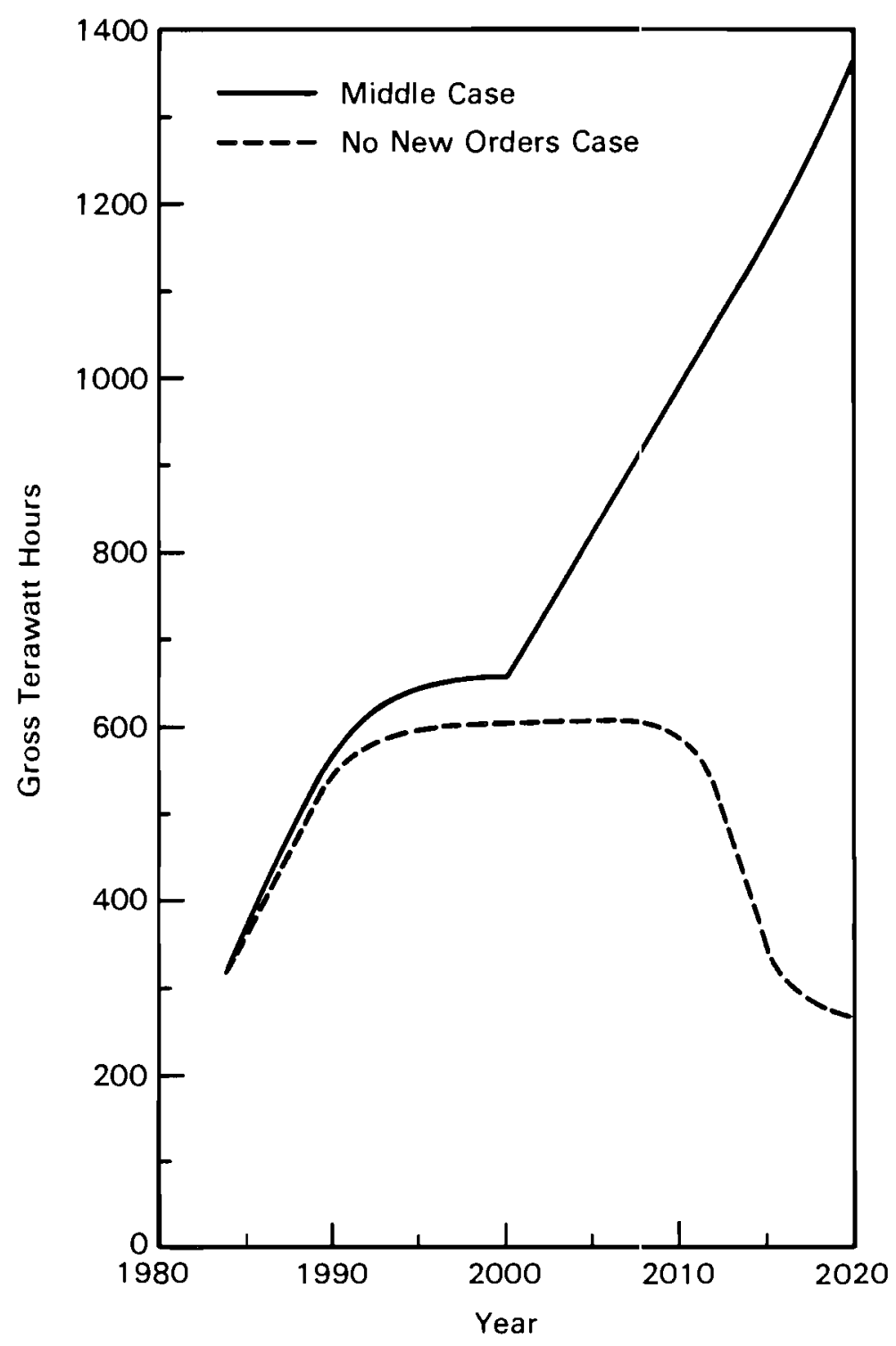

FIGURE 3.3. Projected EIA Nuclear Energy Generation

The maximum at-reactor storage capacity case results are given in Table 3.10 for the four projected data bases: No New Orders with Extended Burnup, No New Orders with Constant Burnup, Middle Case with Extended Burnup, and the Middle Case with Constant Burnup. The time period covers the range from 1985 to the year 2020. The availability of federally-operated storage or repository facilities is not taken into account in these projections. It is 


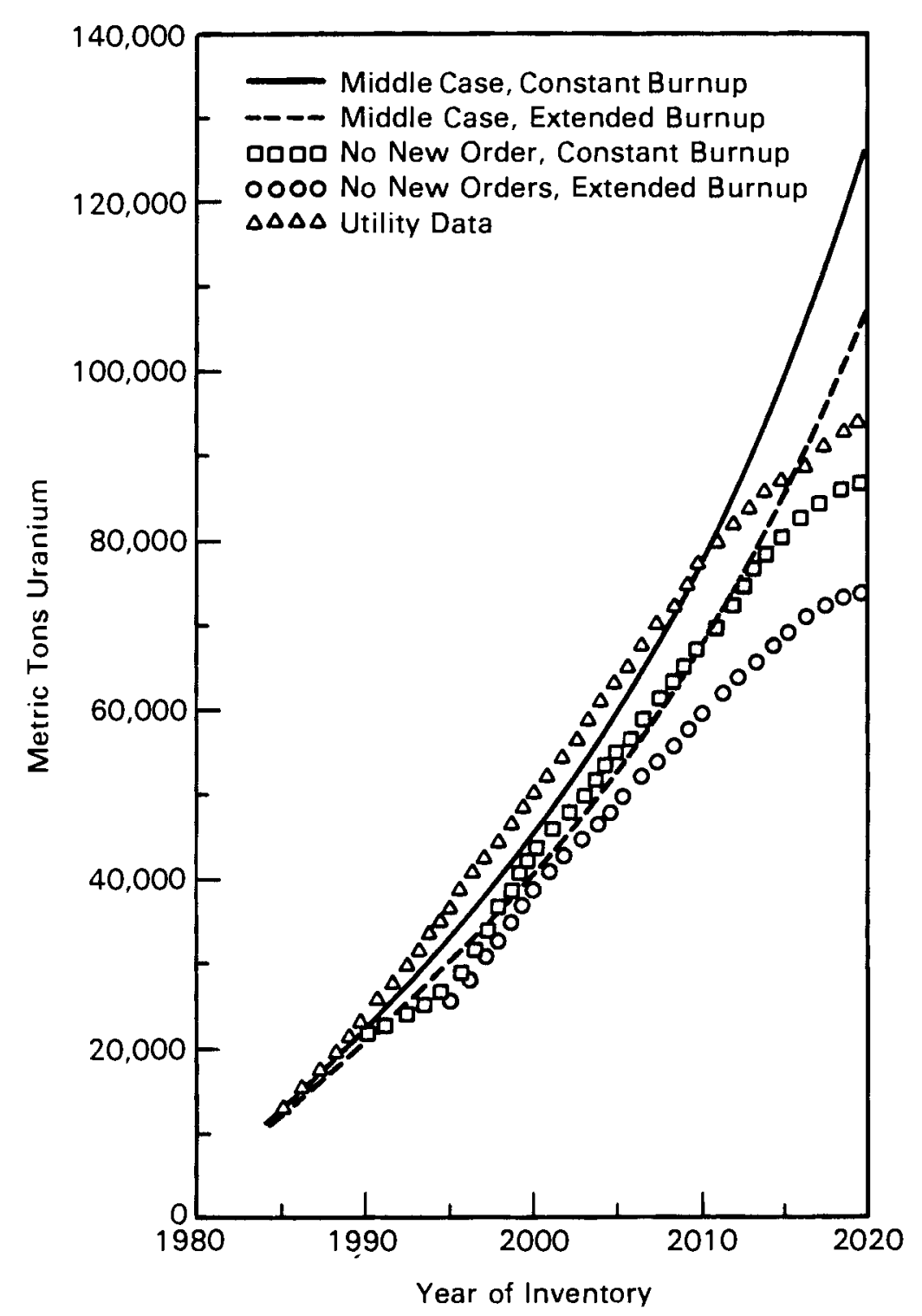

FIGURE 3.4. Cumulative Spent Fuel Inventory for the Four Adjusted Data Bases, and for the Unadjusted Utility Data

expected that the first repository will begin accepting fuel by 1998, which would make the projections in Tables 3.10 and 3.11 unrealistic for that year and beyond.

It is evident that the four adjusted data bases have substantially reduced additional storage requirement needs from utility projections. This reduction is due both to the reduced plant operating capacity factor and (in later years) to the delayed startup assumptions. 
TABLE 3.9. Utility to EIA Required Energy Adjustment Factors--EIA/SFDB

\begin{tabular}{|c|c|c|}
\hline Year & $\begin{array}{c}\text { No New } \\
\text { Orders Case } \\
\end{array}$ & Middle Case \\
\hline 1984 & 0.948 & 0.966 \\
\hline 1985 & 0.955 & 0.912 \\
\hline 1986 & 0.902 & 0.887 \\
\hline 1987 & 0.864 & 0.902 \\
\hline 1988 & 0.855 & 0.934 \\
\hline 1989 & 0.837 & 0.990 \\
\hline 1990 & 0.878 & 1.014 \\
\hline 1991 & 0.885 & 1.000 \\
\hline 1992 & 0.887 & 0.967 \\
\hline 1993 & 0.901 & 0.974 \\
\hline 1994 & 0.919 & 0.987 \\
\hline 1995 & 0.928 & 0.996 \\
\hline 1996 & 0.926 & 0.998 \\
\hline 1997 & 0.920 & 1.010 \\
\hline 1998 & 0.930 & 1.008 \\
\hline 1999 & 0.944 & 1.001 \\
\hline 2000 & 0.943 & 1.002 \\
\hline 2001 & 0.941 & 1.015 \\
\hline 2002 & 0.935 & 0.996 \\
\hline 2003 & 0.937 & 0.986 \\
\hline 2004 & 0.942 & 0.982 \\
\hline 2005 & 0.941 & 0.975 \\
\hline 2006 & 0.936 & 0.969 \\
\hline 2007 & 0.941 & 0.997 \\
\hline 2008 & 0.934 & 0.987 \\
\hline 2009 & 0.940 & 0.967 \\
\hline 2010 & 0.939 & 0.990 \\
\hline 2011 & 0.855 & 0.952 \\
\hline 2012 & 0.882 & 0.917 \\
\hline 2013 & 0.913 & 0.892 \\
\hline 2014 & 0.961 & 0.899 \\
\hline 2015 & 0.969 & 0.907 \\
\hline 2016 & 0.933 & 0.900 \\
\hline 2017 & 0.895 & 0.913 \\
\hline 2018 & 0.915 & 0.913 \\
\hline 2019 & 51 & 0.911 \\
\hline 2020 & 53 & 0.917 \\
\hline
\end{tabular}

The maximum at-reactor storage capacity plus transshipment case results are given in Table 3.11. Again, a substantially reduced additional storage requirement need is evident under the adjusted data base energy generation and capacity assumptions. 
TABLE 3.10. Cumulative Storage Requirements--Maximum AR Storage (MTIHM)

\begin{tabular}{|c|c|c|c|c|c|}
\hline \multirow[b]{2}{*}{ Year } & \multirow{2}{*}{$\begin{array}{l}\text { Utility } \\
\text { Base }\end{array}$} & \multicolumn{2}{|c|}{ No New Orders } & \multicolumn{2}{|c|}{ Middle Case } \\
\hline & & Ext. Burnup & Const. Burnup & Ext. Burnup & Const. Burnup \\
\hline 1985 & 1 & 3 & 5 & 0 & 3 \\
\hline 1986 & 50 & 30 & 41 & 22 & 32 \\
\hline 1987 & 126 & 54 & 77 & 27 & 43 \\
\hline 1988 & 271 & 160 & 211 & 116 & 144 \\
\hline 1989 & 521 & 323 & 397 & 249 & 313 \\
\hline 1990 & 759 & 449 & 560 & 334 & 435 \\
\hline 1991 & 1148 & 687 & 850 & 600 & 738 \\
\hline 1992 & 1633 & 973 & 1199 & 771 & 1017 \\
\hline 1993 & 2056 & 1231 & 1566 & 1094 & 1349 \\
\hline 1994 & 2590 & 1659 & 2083 & 1343 & 1800 \\
\hline 1995 & 3392 & 2024 & 2631 & 1789 & 2287 \\
\hline 1996 & 4156 & 2478 & 3279 & 2049 & 2832 \\
\hline 1997 & 5172 & 2994 & 4055 & 2637 & 3604 \\
\hline 1998 & 6601 & 3563 & 4946 & 3203 & 4421 \\
\hline 1999 & 7888 & 4235 & 6089 & 3641 & 5373 \\
\hline 2000 & 9332 & 5025 & 7296 & 4591 & 6661 \\
\hline 2001 & 10947 & 5869 & 8444 & 5206 & 7770 \\
\hline 2002 & 12751 & 6796 & 9899 & 6462 & 9260 \\
\hline 2003 & 14811 & 7811 & 11319 & 7210 & 10682 \\
\hline 2004 & 16671 & 8717 & 12845 & 8322 & 12178 \\
\hline 2005 & & 9736 & 14405 & 9354 & 13797 \\
\hline 2006 & & 10823 & 16074 & 10174 & 15642 \\
\hline 2007 & & 11906 & 17815 & 11410 & 17035 \\
\hline 2008 & & 13214 & 19595 & 12282 & 18514 \\
\hline 2009 & & 14464 & 21586 & 13681 & 20632 \\
\hline 2010 & & 15999 & 23829 & 14513 & 22053 \\
\hline 2011 & & 17649 & 26269 & 15702 & 23943 \\
\hline 2012 & & 19038 & 28261 & 16873 & 26004 \\
\hline 2013 & & 20229 & 29920 & 18089 & 28392 \\
\hline 2014 & & 21313 & 31507 & 19361 & 30405 \\
\hline 2015 & & 21818 & 32230 & 20148 & 32129 \\
\hline 2016 & & 22824 & 33719 & 21349 & 34513 \\
\hline 2017 & & 23783 & 34939 & 22323 & 36961 \\
\hline 2018 & & 24383 & 35875 & 23145 & 38727 \\
\hline 2019 & & 25148 & 36841 & 24048 & 41043 \\
\hline 2020 & & 25784 & 37655 & 25035 & 43512 \\
\hline
\end{tabular}

In many years, Table 3.10 shows a higher storage requirement for the No New Orders Case than for the Middle case for both extended and constant burnup scenarios. Since the Middle Case has a larger energy generation, one would expect that it would have a higher storage requirement than the No New Orders Case. This does not occur because the Middle Case has more reactors than the 
TABLE 3.11. Cumulative Storage Requirements-..Maximum AR Storage Plus Transshipment (MTIHM)

\begin{tabular}{|c|c|c|c|c|c|}
\hline \multirow[b]{2}{*}{ Year } & \multirow{2}{*}{$\begin{array}{c}\text { Utility } \\
\text { Base } \\
\end{array}$} & \multicolumn{2}{|c|}{ No New Orders } & \multicolumn{2}{|c|}{ Middle Case } \\
\hline & & Ext. Burnup & Const. Burnup & Ext. Burnup & Const. Burnup \\
\hline 1985 & 0 & 0 & 0 & 0 & 0 \\
\hline 1986 & 0 & 0 & 0 & 0 & 0 \\
\hline 1987 & 31 & 17 & 20 & 5 & 7 \\
\hline 1988 & 57 & 39 & 44 & 19 & 27 \\
\hline 1989 & 89 & 63 & 70 & 41 & 53 \\
\hline 1990 & 92 & 63 & 70 & 41 & 53 \\
\hline 1991 & 258 & 110 & 133 & 100 & 117 \\
\hline 1992 & 452 & 155 & 238 & 114 & 166 \\
\hline 1993 & 729 & 241 & 446 & 201 & 293 \\
\hline 1994 & 1100 & 509 & 842 & 309 & 546 \\
\hline 1995 & 1719 & 797 & 1323 & 532 & 858 \\
\hline 1996 & 2523 & 1201 & 1850 & 726 & 1315 \\
\hline 1997 & 3529 & 1599 & 2530 & 1092 & 1955 \\
\hline 1998 & 4952 & 2130 & 3411 & 1627 & 2744 \\
\hline 1999 & 6357 & 2768 & 4437 & 2044 & 3633 \\
\hline 2000 & 7919 & 3434 & 5654 & 2988 & 4784 \\
\hline 2001 & 9543 & 4262 & 6778 & 3444 & 5690 \\
\hline 2002 & 11283 & 5092 & 7991 & 4370 & 6786 \\
\hline 2003 & 13400 & 6122 & 9488 & 5003 & 8051 \\
\hline 2004 & 15118 & 6969 & 11016 & 5847 & 9577 \\
\hline 2005 & & 7919 & 12570 & 6823 & 11263 \\
\hline 2006 & & 9057 & 14225 & 7613 & 13173 \\
\hline 2007 & & 10123 & 15957 & 8926 & 14610 \\
\hline 2008 & & 11419 & 17796 & 9875 & 16113 \\
\hline 2009 & & 12685 & 19845 & 11366 & 18139 \\
\hline 2010 & & 14187 & 22042 & 12266 & 19529 \\
\hline 2011 & & 15888 & 24456 & 13490 & 21296 \\
\hline 2012 & & 17230 & 26339 & 14844 & 23084 \\
\hline 2013 & & 18426 & 28000 & 16129 & 25165 \\
\hline 2014 & & 19558 & 29659 & 17439 & 26955 \\
\hline 2015 & & 20074 & 30412 & 18251 & 28384 \\
\hline 2016 & & 21102 & 31960 & 19494 & 30663 \\
\hline 2017 & & 22089 & 33262 & 20445 & 33407 \\
\hline 2018 & & 22679 & 34240 & 21240 & 34972 \\
\hline 2019 & & 23450 & 35254 & 22133 & 37504 \\
\hline 2020 & & 24068 & 36090 & 23104 & 40106 \\
\hline
\end{tabular}

No New Orders Case and the per-reactor energy generation is actually higher in the No New.Orders Case than it is in the Middle Case. In this situation, the older reactors with limited storage generate more energy, and hence discharge 
more fuel in the No New Orders Case. As discussed in Section 1.0, the variables in the case scenarios for current and pipeline reactors reflect variation in plant operation at the same plant.

In the period after 1995, the Middle Case has up to nine more pipeline reactors operating than in the No New Orders Case. In addition, it has generic reactor additions starting in the year 2000. Discharges from reactors which started up before 1985 are higher in the No New Orders Case than in the Middle Case. Since these older reactors have lower storage capacity than generic reactors and the Middle Case additional pipeline reactors, the No New Orders Case storage requirements remain above Middle Case requirements.

The No New Orders requirement excess above the Middle Case reaches a maximum of 2165 MTIHM in 2012 for the extended burnup scenario. This further increase is due to the difference in shutdown dates for reactors which started up in 1984 or before. For example, the No New Orders Case has 18 reactors shutting down in 2011. It would have been unrealistic to introduce 18 generic reactors in the Middle Case to make up for the large decrease in capacity in 2011. Therefore the Middle Case shutdown dates where moved to an earlier period for some of these reactors. A similar procedure was followed for other years in developing the Middle Case data base in order to smooth out the addition of generic reactor capacity. The result of the advanced shutdown dates for historic reactors is that the Middle Case low storage capacity reactors are removed sooner than those same reactors in the No New Orders Case. Since they are replaced with high storage capacity generic reactors, the No New Orders Case storage requirements remain above the Middle Case extended burnup cases in the 2006 to 2020 time period. In the constant burnup cases, the greater total fuel inventory compared to the extended burnup cases causes the Middle Case requirement to finally exceed the No New Orders Case requirements in 2016. A similar crossover would occur after 2020 with the extended burnup cases.

The additional storage requirements taking transshipment into account are shown in Table 3.11 for the four projected data bases and for the utility data base. A comparison of Tables 3.10 and 3.11 shows that transshipment is an effective means of reducing additional storage requirements. Storage requirements based on the information from Tables 3.10 and 3.11 are illustrated in 
Figures 3.5 and 3.6. The No New Orders Case storace requirements with transshipment are higher than those for the Middle Case for the same reasons cited for the maximum at-reactor case without transshipment. The tirend is the same for both maximum at-reactor storage with transshipment and without transshipment. The No New Orders Case requirements remain above Middle Case requirements for the constant burnup scenario until 2016 for the maximum at-reactor storage case and 2017 for the maximum at-reactor storage plus transshipment case. At this time, a crossover occurs and the higher discharge of the Middle Case finally results in higher requirements for the Middle Case. Such a crossover occurs for the extended burnup scenario only after 2020. 


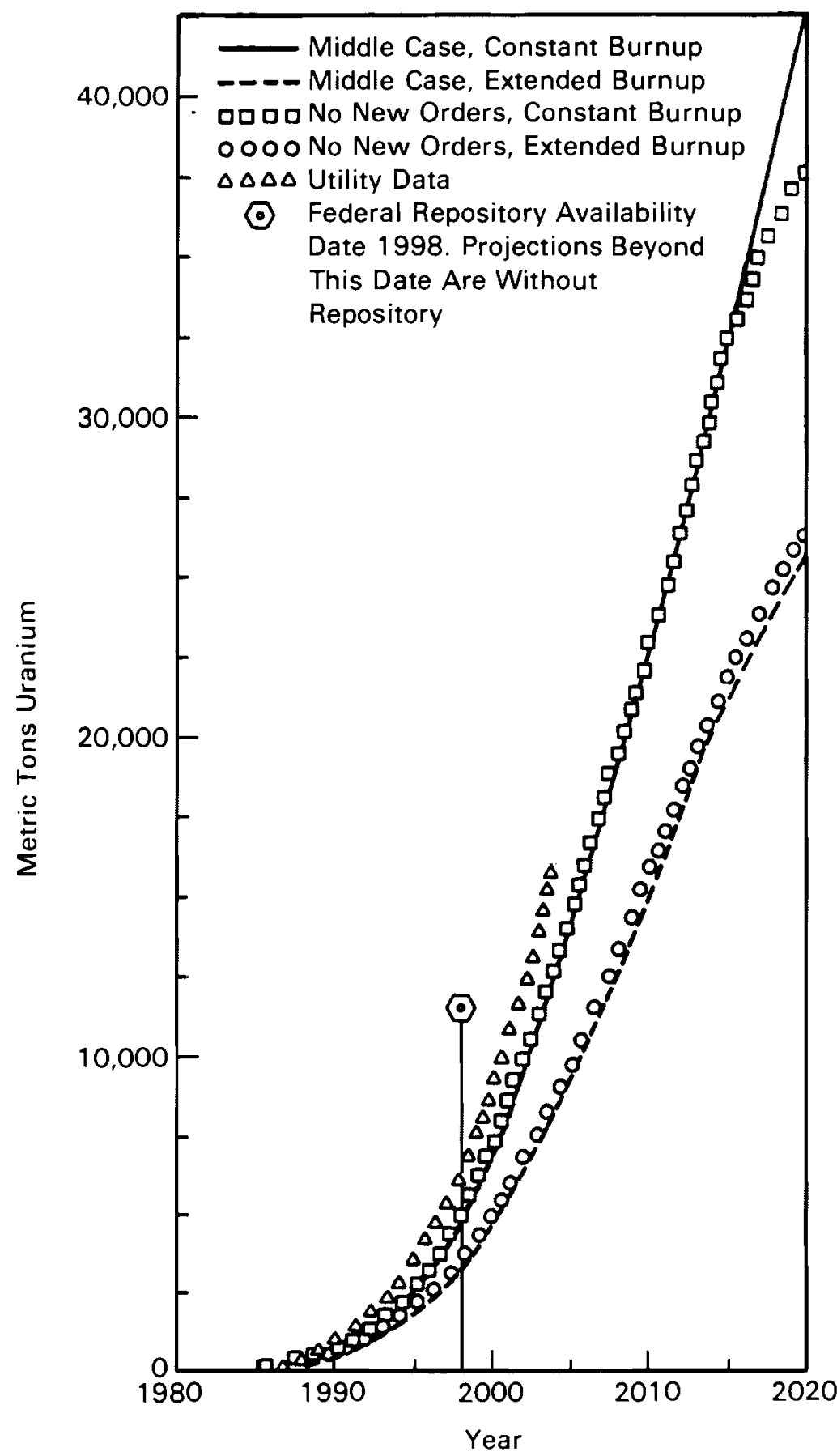

FIGURE 3.5. Cumulative Additional Storage Requirement--Maximum At-Reactor Storage 


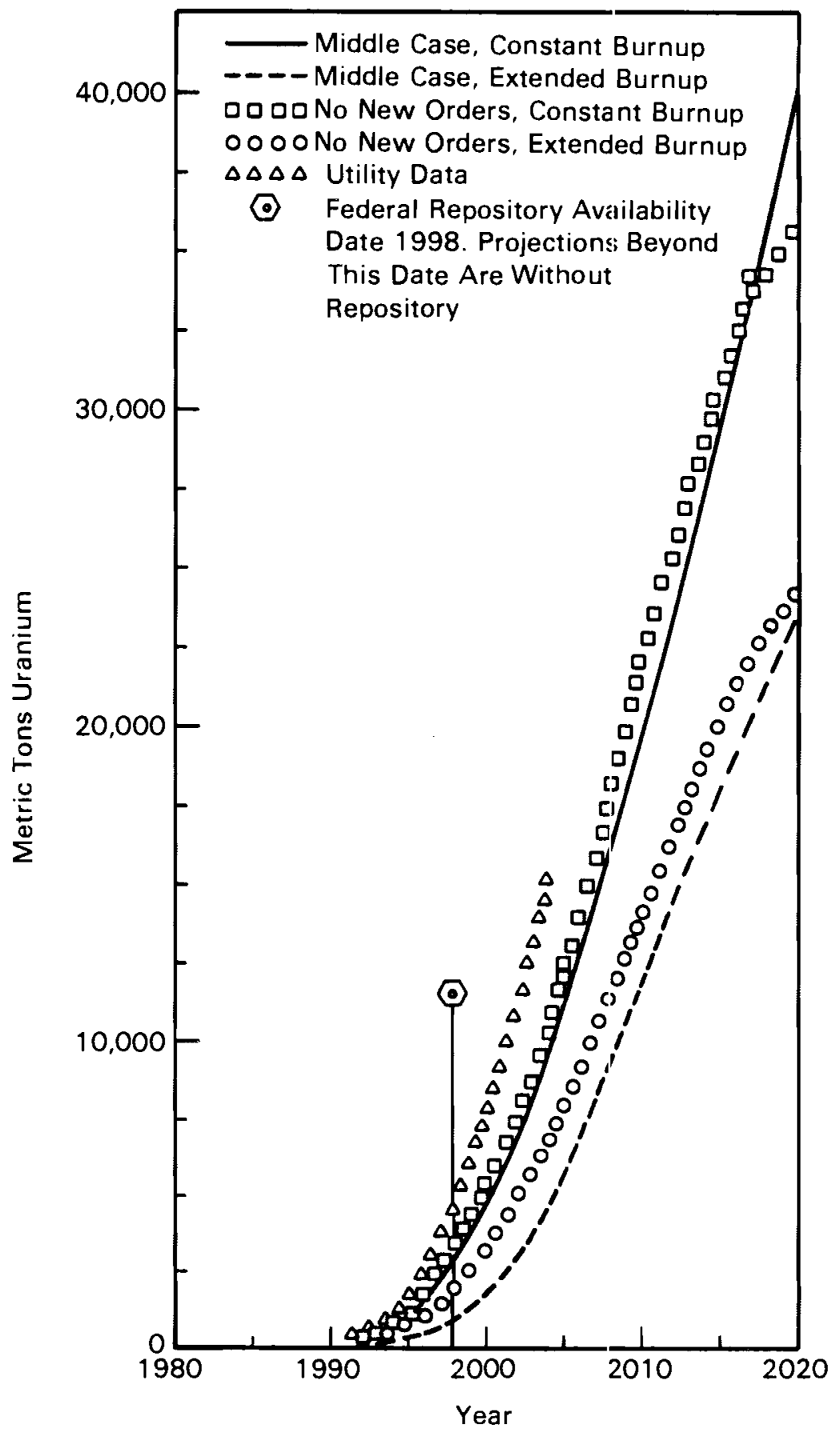

FIGURE 3.6. Cumulative Additional Storage Requirement--Maximum At-Reactor Storage Plus Transshipment 


\subsection{REFERENCES}

DOE. 1985a. Spent Fuel Storage Requirements: An Update of DOE/RL-84-1. DOE/RL-85-2, U.S. Department of Energy, Richland Operations Office, Richl and, Washington.

DOE. 1985b. Spent Fuel and Radioactive Waste Inventories, Projections, and Characteristics, D0E/RW-0006, Rev.1, U.S. Department of Energy, Office of Nuclear Energy, Washington, D.C.

Heeb, C. M., R. A. Libby and G. M. Holter. 1985. Reactor-Specific Spent Fuel Discharge Projections: 1984 to 2020. PNL-5396, Pacific Northwest

Laboratory, Richland, Washington.

Holter, G. M. et al. 1986. A Method for Assigning Sites to Projected Generic Nuclear Power Plants. PNL-5870, Pacific Northwest Laboratory, Richland, Washington.

Mayes, F. et al. 1985. Commercial Nuclear Power: Prospects for the United States and the World. DOE/EIA-0438 (85), U.S. Department of Energy, Washington, D.C.

Moden, R. et al. 1985. World Nuclear Fuel Cycle Requirements 1985. DOE/EIA0436 (85), U.S. Department of Energy, Washington, D.C. 



\section{APPENDIX A}

DETAILED DATA BASE RESULTS 
APPENDIX A

\section{DETAILED DATA BASE RESULTS}

The following tables provide detailed results for three scenarios: Utility, No New Orders Case, and Middle Case. Table A.1 gives startup and shutdown dates for reactors in each scenario. Table A.2 gives names and startup dates for the Middle Case generic reactors. Tables A.3 to A.8 give spent fuel discharge projections by reactor similar to information published by DOE (1985a) for the Utility Data Base. The Middle Case has been selected by OCRWM as the projection to be used for planning purposes. The Middle Case with extended burnup is the EIA base case assumption for 1985. Therefore Tables A.3 through A.8 show the Middle Case with extended burnup.

Table

Number

Title

A.1 Startup and Shutdown Dates for Utility, No New Orders and Middle Case

A.2 Startup and Shutdown Dates of Middle Case Generic Reactors

A.3 Middle Case (with Extended Burnup) 1984 Inventory and Projected Annual Reactor Discharges

A.4 Middle Case (with Extended Burnup) 1984 and Projected Inventories

A.5 Middle Case (with Extended Burnup) Maximum At-Reactor Capacity Projected Annual Additional Storage Requirements

A.6 Middle Case (with Extended Burnup) Maximum At-Reactor Capacity Projected Cumulative Storage Requirements

A.7 Middle Case (with Extended Burnup) Maximum At-Reactor Capacity - Plus Transshipment - Projected Annual Storage Requirements

A.8 Middle Case (with Extended Burnup) Maximum At-Reactor Capacity - Plus Transshipment - Projected Cumulative Storage Requirements 
TABLE A.1. Startup and Shutdown Dates for Utility, No New Orders, and Middle Cases

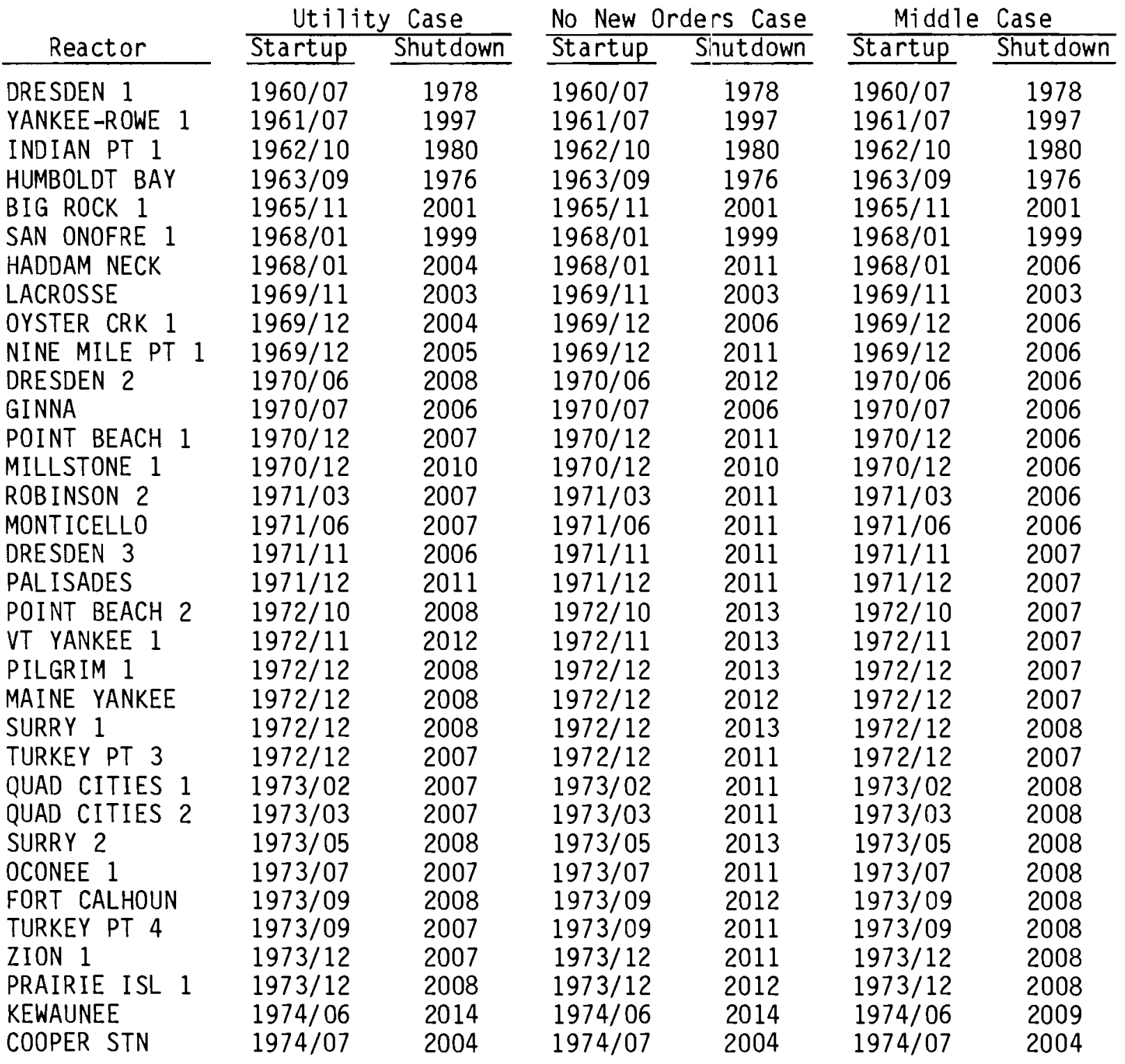


TABLE A.1. Startup and Shutdown Dates for Utility, No New Orders, and Middle Cases (contd)

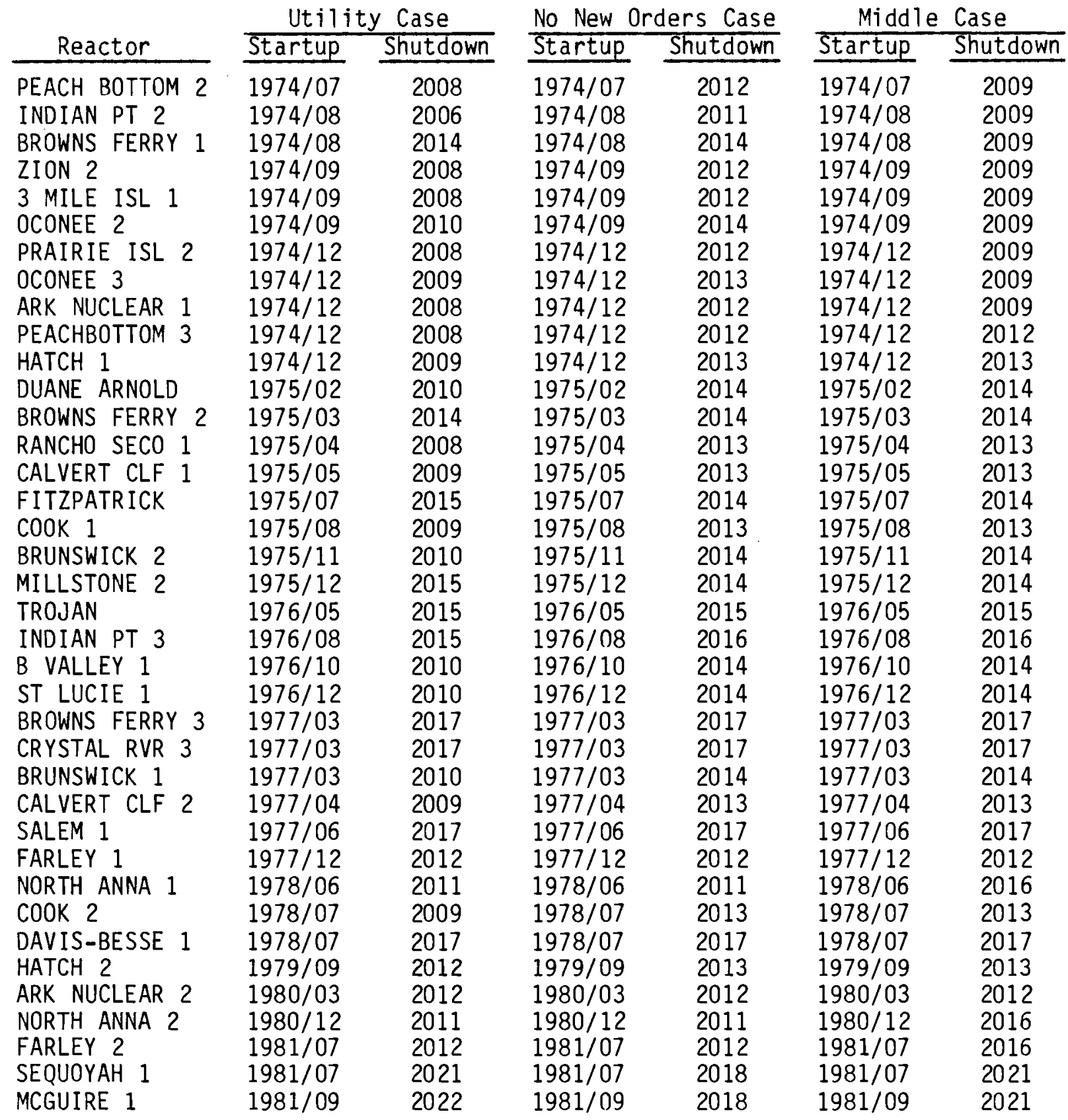


TABLE A.1. Startup and Shutdown Dates for Utili:y, No New Orders, and Middle Cases (contd)

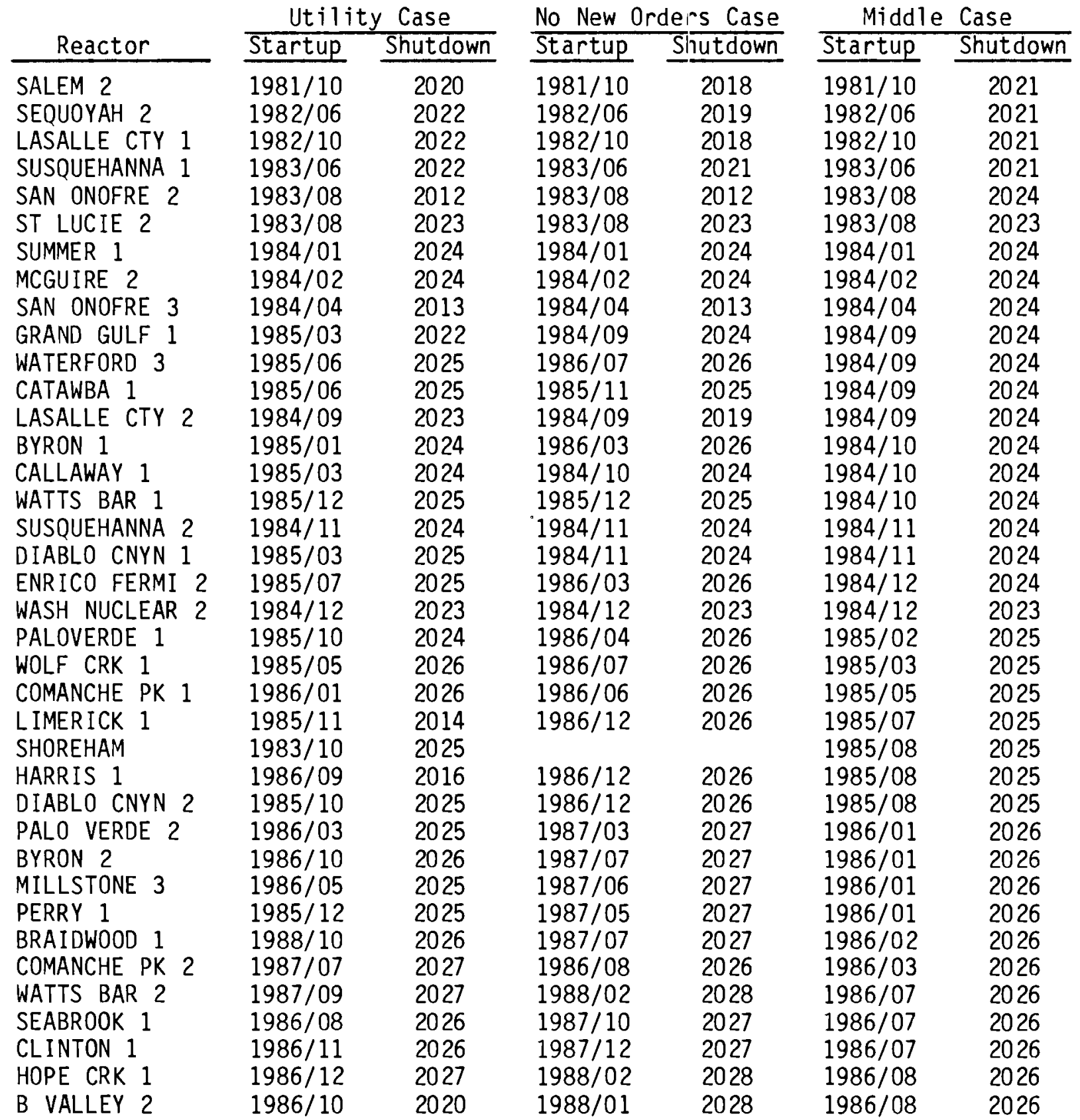


TABLE A.1. Startup and Shutdown Dates for Utility, No New Orders, and Middle Cases (contd)

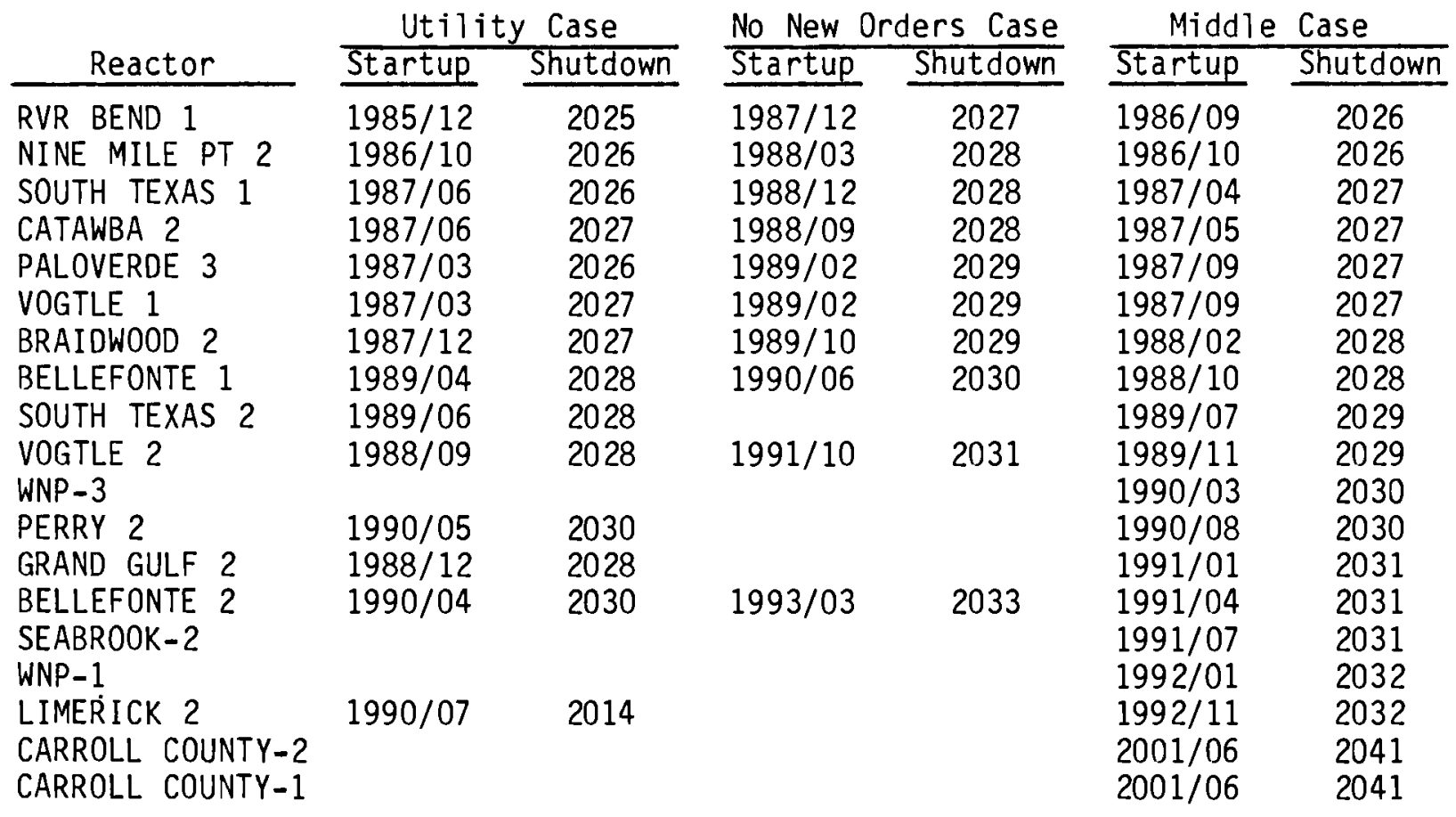


TABLE A.2. Startup and Shutdown Dates of Middle Case Generic Reactors

\begin{tabular}{ccccc} 
Reactor Name & Region & & Startup & Shutdown \\
\cline { 2 - 2 } P010101 & 4 & & $2001 / 01$ & $2041 / 01$ \\
P010201 & 5 & & $2001 / 01$ & $2041 / 01$ \\
B010101 & 2 & $2001 / 01$ & $2041 / 01$ \\
P010305 & 4 & $2001 / 05$ & $2041 / 05$ \\
B010209 & 3 & $2001 / 09$ & $2041 / 09$ \\
B020101 & 9 & $2002 / 01$ & $2042 / 01$ \\
P020301 & 4 & $2002 / 01$ & $2042 / 01$ \\
P020201 & 5 & $2002 / 01$ & $2042 / 01$ \\
P020101 & 3 & $2002 / 01$ & $2042 / 01$ \\
P020403 & 2 & $2002 / 03$ & $2042 / 03$ \\
B020208 & 4 & $2002 / 08$ & $2042 / 08$ \\
P030101 & 9 & $2003 / 01$ & $2043 / 01$ \\
P030201 & 6 & $2003 / 01$ & $2043 / 01$ \\
P030301 & 4 & $2003 / 01$ & $2043 / 01$ \\
B030101 & 4 & $2003 / 01$ & $2043 / 01$ \\
P030403 & 1 & $2003 / 03$ & $2043 / 03$ \\
B030208 & 5 & $2003 / 08$ & $2043 / 08$ \\
P040301 & 4 & $2004 / 01$ & $2044 / 01$ \\
P040201 & 3 & $2004 / 01$ & $2044 / 01$ \\
P040101 & 5 & $2004 / 01$ & $2044 / 01$ \\
B040101 & 6 & $2004 / 01$ & $2044 / 01$ \\
P040402 & 4 & $2004 / 02$ & $2044 / 02$ \\
B040208 & 4 & $2004 / 08$ & $2044 / 08$ \\
P050301 & 2 & $2005 / 01$ & $2045 / 01$ \\
P050201 & 4 & $2005 / 01$ & $2045 / 01$ \\
P050101 & 5 & $2005 / 01$ & $2045 / 01$ \\
P050401 & 9 & $2005 / 01$ & $2045 / 01$ \\
B050101 & 5 & $2005 / 01$ & $2045 / 01$ \\
B050205 & 3 & $2005 / 05$ & $2045 / 05$ \\
P050508 & 3 & $2005 / 08$ & $2045 / 08$ \\
B060101 & 4 & $2006 / 01$ & $2046 / 01$ \\
P060101 & 4 & $2006 / 01$ & $2046 / 01$ \\
P060201 & 7 & $2006 / 01$ & $2046 / 01$ \\
P060301 & 5 & $2006 / 01$ & $2046 / 01$ \\
B060202 & 7 & $2006 / 02$ & $2046 / 02$ \\
P060405 & 6 & $2006 / 05$ & $2046 / 05$ \\
P070301 & 5 & $2007 / 01$ & $2047 / 01$ \\
P070501 & 3 & $2007 / 01$ & $2047 / 01$ \\
B070101 & 2 & $2007 / 01$ & $2047 / 01$ \\
B070201 & 9 & $2007 / 01$ & $2047 / 01$ \\
P070401 & 4 & $2007 / 01$ & $2047 / 01$
\end{tabular}


TABLE A.2. Startup and Shutdown Dates of Middle Case Generic Reactors (contd)

\begin{tabular}{ccccc} 
Reactor Name & Region & & Startup & Shutdown \\
\cline { 2 - 2 } P070201 & 1 & & $2007 / 01$ & $2047 / 01$ \\
P070101 & 4 & & $2007 / 01$ & $2047 / 01$ \\
B070307 & 4 & $2007 / 07$ & $2047 / 07$ \\
P070612 & 2 & $2007 / 12$ & $2047 / 12$ \\
B080301 & 4 & $2008 / 01$ & $2048 / 01$ \\
P080701 & 5 & $2008 / 01$ & $2048 / 01$ \\
P080201 & 4 & $2008 / 01$ & $2048 / 01$ \\
B080101 & 5 & $2008 / 01$ & $2048 / 01$ \\
P080101 & 9 & $2008 / 01$ & $2048 / 01$ \\
P080401 & 9 & $2008 / 01$ & $2048 / 01$ \\
P080601 & 6 & $2008 / 01$ & $2048 / 01$ \\
P080501 & 4 & $2008 / 01$ & $2048 / 01$ \\
B080201 & 1 & $2008 / 01$ & $2048 / 01$ \\
P080301 & 5 & $2008 / 01$ & $2048 / 01$ \\
B080406 & 3 & $2008 / 06$ & $2048 / 06$ \\
P080809 & 3 & $2008 / 09$ & $2048 / 09$ \\
P090101 & 4 & $2009 / 01$ & $2049 / 01$ \\
B090201 & 6 & $2009 / 01$ & $2049 / 01$ \\
B090501 & 5 & $2009 / 01$ & $2049 / 01$ \\
B090301 & 4 & $2009 / 01$ & $2049 / 01$ \\
B090101 & 5 & $2009 / 01$ & $2049 / 01$ \\
P091001 & 5 & $2009 / 01$ & $2049 / 01$ \\
B090401 & 9 & $2009 / 01$ & $2049 / 01$ \\
P090801 & 4 & $2009 / 01$ & $2049 / 01$ \\
P090701 & 3 & $2009 / 01$ & $2049 / 01$ \\
P090601 & 9 & $2009 / 01$ & $2049 / 01$ \\
P090501 & 2 & $2009 / 01$ & $2049 / 01$ \\
P090401 & 5 & $2009 / 01$ & $2049 / 01$ \\
P090301 & 4 & $2009 / 01$ & $2049 / 01$ \\
P090901 & 7 & $2009 / 01$ & $2049 / 01$ \\
P090201 & 1 & $2009 / 01$ & $2049 / 01$ \\
P091108 & 4 & $2009 / 08$ & $2049 / 08$ \\
B090611 & 4 & $2009 / 11$ & $2049 / 11$ \\
P100601 & 4 & $2010 / 01$ & $2050 / 01$ \\
P100501 & 2 & $2010 / 01$ & $2050 / 01$ \\
P100401 & 1 & $2010 / 01$ & $2050 / 01$ \\
P100301 & 4 & $2010 / 01$ & $2050 / 01$ \\
P100201 & 5 & $2010 / 01$ & $2050 / 01$ \\
B100201 & 9 & $2010 / 01$ & $2050 / 01$ \\
P100101 & 6 & $2010 / 01$ & $2050 / 01$
\end{tabular}


TABLE A.2. Startup and Shutdown Dates of Middle Case Generic Reactors (contd)

\begin{tabular}{lcccc} 
Reactor Name & Region & & Startup & Shutdown \\
\cline { 2 - 2 } B100101 & 2 & & $2010 / 01$ & $2050 / 01$ \\
B100302 & 3 & & $2010 / 02$ & $2050 / 02$ \\
P100705 & 9 & $2010 / 05$ & $2050 / 05$ \\
B110301 & 1 & $2011 / 01$ & $2051 / 01$ \\
P110401 & 4 & $2011 / 01$ & $2051 / 01$ \\
P110501 & 3 & $2011 / 01$ & $2051 / 01$ \\
P110601 & 5 & $2011 / 01$ & $2051 / 01$ \\
P110201 & 4 & $2011 / 01$ & $2051 / 01$ \\
P110301 & 5 & $2011 / 01$ & $2051 / 01$ \\
P110701 & 6 & $2011 / 01$ & $2051 / 01$ \\
P110101 & 4 & $2011 / 01$ & $2051 / 01$ \\
B110201 & 5 & $2011 / 01$ & $2051 / 01$ \\
B110101 & 4 & $2011 / 01$ & $2051 / 01$ \\
B110406 & 4 & $2011 / 06$ & $2051 / 06$ \\
P110812 & 4 & $2011 / 12$ & $2051 / 12$ \\
B120201 & 5 & $2012 / 01$ & $2052 / 01$ \\
B120101 & 6 & $2012 / 01$ & $2052 / 01$ \\
P120201 & 9 & $2012 / 01$ & $2052 / 01$ \\
P120101 & 2 & $2012 / 01$ & $2052 / 01$ \\
P120301 & 4 & $2012 / 01$ & $2052 / 01$ \\
P120401 & 5 & $2012 / 01$ & $2052 / 01$ \\
P120503 & 1 & $2012 / 03$ & $2052 / 03$ \\
B120308 & 3 & $2012 / 08$ & $2052 / 08$ \\
P130101 & 3 & $2013 / 01$ & $2053 / 01$ \\
P130501 & 10 & $2013 / 01$ & $2053 / 01$ \\
P130201 & 4 & $2013 / 01$ & $2053 / 01$ \\
P130301 & 7 & $2013 / 01$ & $2053 / 01$ \\
P130401 & 5 & $2013 / 01$ & $2053 / 01$ \\
P130601 & 4 & $2013 / 01$ & $2053 / 01$ \\
B130301 & 2 & $2013 / 01$ & $2053 / 01$ \\
B130201 & 7 & $2013 / 01$ & $2053 / 01$ \\
B130101 & 4 & $2013 / 01$ & $2053 / 01$ \\
P130702 & 7 & $2013 / 02$ & $2053 / 02$ \\
B140201 & 4 & $2014 / 01$ & $2054 / 01$ \\
P140301 & 4 & $2014 / 01$ & $2054 / 01$ \\
P140201 & 9 & $2014 / 01$ & $2054 / 01$ \\
P140401 & 5 & $2014 / 01$ & $2054 / 01$ \\
P140101 & 2 & & $2014 / 01$ & $2054 / 01$ \\
B140101 & 9 & $2014 / 01$ & $2054 / 01$ \\
P140505 & 3 & & $2014 / 05$ & $2054 / 05$
\end{tabular}


TABLE A.2. Startup and Shutdown Dates of Middle Case Generic Reactors (contd)

\begin{tabular}{ccccc} 
Reactor Name & Region & & Startup & Shutdown \\
\cline { 2 - 2 } B140308 & 5 & & $2014 / 08$ & $2054 / 08$ \\
B150201 & 4 & & $2015 / 01$ & $2055 / 01$ \\
P150501 & 4 & $2015 / 01$ & $2055 / 01$ \\
P150301 & 5 & $2015 / 01$ & $2055 / 01$ \\
P150201 & 8 & $2015 / 01$ & $2055 / 01$ \\
P150101 & 4 & $2015 / 01$ & $2055 / 01$ \\
B150101 & 4 & $2015 / 01$ & $2055 / 01$ \\
P150401 & 5 & $2015 / 01$ & $2055 / 01$ \\
P150608 & 3 & $2015 / 08$ & $2055 / 08$ \\
B150311 & 3 & $2015 / 11$ & $2055 / 11$ \\
P160501 & 6 & $2016 / 01$ & $2056 / 01$ \\
P160301 & 2 & $2016 / 01$ & $2056 / 01$ \\
B160101 & 5 & $2016 / 01$ & $2056 / 01$ \\
B160201 & 1 & $2016 / 01$ & $2056 / 01$ \\
P160201 & 4 & $2016 / 01$ & $2056 / 01$ \\
P160401 & 9 & $2016 / 01$ & $2056 / 01$ \\
P160101 & 5 & $2016 / 01$ & $2056 / 01$ \\
P160606 & 4 & $2016 / 06$ & $2056 / 06$ \\
B160309 & 4 & $2016 / 09$ & $2056 / 09$ \\
P170101 & 5 & $2017 / 01$ & $2057 / 01$ \\
B170101 & 6 & $2017 / 01$ & $2057 / 01$ \\
P170201 & 5 & $2017 / 01$ & $2057 / 01$ \\
P170301 & 3 & $2017 / 01$ & $2057 / 01$ \\
P170407 & 4 & $2017 / 07$ & $2057 / 07$ \\
B170211 & 2 & $2017 / 11$ & $2057 / 11$ \\
P180501 & 2 & $2018 / 01$ & $2058 / 01$ \\
P180401 & 4 & $2018 / 01$ & $2058 / 01$ \\
P180201 & 1 & $2018 / 01$ & $2058 / 01$ \\
B180201 & 4 & $2018 / 01$ & $2058 / 01$ \\
P180301 & 5 & $2018 / 01$ & $2058 / 01$ \\
B180101 & 9 & $2018 / 01$ & $2058 / 01$ \\
P180101 & 7 & $2018 / 01$ & $2058 / 01$ \\
P180605 & 9 & $2018 / 05$ & $2058 / 05$ \\
B180308 & 5 & $2018 / 08$ & $2058 / 08$ \\
P190301 & 5 & $2019 / 01$ & $2059 / 01$ \\
P190201 & 6 & $2019 / 01$ & $2059 / 01$ \\
P190101 & 4 & $2019 / 01$ & $2059 / 01$ \\
B190101 & 3 & $2019 / 01$ & $2059 / 01$ \\
P190406 & 3 & $2019 / 01$ & $2059 / 01$
\end{tabular}


TABLE A.2. Startup and Shutdown Dates of Middle Case Generic Reactors (contd)

\begin{tabular}{|c|c|c|c|}
\hline Reactor Name & Region & Startup & Shutdown \\
\hline B190209 & 4 & $2019 / 09$ & $2059 / 09$ \\
\hline P200101 & 4 & $2020 / 01$ & $2060 / 01$ \\
\hline P200201 & 5 & $2020 / 01$ & $2060 / 01$ \\
\hline P200301 & 4 & $2020 / 01$ & $2060 / 01$ \\
\hline B200101 & 5 & $2020 / 01$ & $2060 / 01$ \\
\hline P200407 & 1 & $2020 / 07$ & $2060 / 07$ \\
\hline B200211 & 4 & $2020 / 11$ & $2060 / 11$ \\
\hline
\end{tabular}

Note: The generic reactor name consists of four identification fields:

- The first character denotes reactor type. $P$ for PWR and B for BWR

- The next two characters are the last two digits of the startup year

- The second set of two characters denotes the sequence within a year

- The last two characters denote the startup month. 
TABLE A.3. Middle Case (with Extended Burnup) 1984 Inventory and Projected Annual Reactor Discharges

REACTOR

ARK NUCLEAR 1 ARK NUCLEAR 2

B VALLEY 1

B VALLEY 2

BELLEONTE 1

BELLEFONTE 2

BIG ROCK 1

BRAIDWODD 1

BRAIDWODD 2

BROWNS FERRY1

BROWNS FERRY2

BROWNS FERRY3

BRUNSWICK 1

BRUNSWICK 2

BYRON 1

BYRON 2

CALLAMAY 1

CALVERT CLF 1

CALVERT CLF 2

CARROLL COUNTY-1

CARROLL COUNTY-2

CATAMBA 1

CATAMBA 2

CLINTON 1

COMANCHE PK 1

COMANCHE PK 2

COOK 1

cook 2

COOPER STN

CRYSTAL RVR 3

DAVIS-BESSE 1

DIABLO CNYN 1

DIABLD CNYN 2

DRESDEN 1

DRESDEN 2

DRESDEN 3

DUANE ARNOLD

ENRICO FERMI2

FARLEY 1

FARLEY 2

FITZPATRICK

FORT CALHOUN

GINNA

GRAND GULF 1

GRAND GULF 2

HADDAM NECK

HARRIS 1

HATCH 1
Inv Assemblies

Inv

MTIHM

$\frac{1984}{384} \frac{1985}{9} \frac{1986}{51} \frac{1987}{9} \frac{1988}{57} \frac{1989}{g} \frac{1999}{48} \frac{1991}{64} \frac{1992}{1993} \frac{1994}{66}: \overline{1984} \frac{1985}{178} \frac{1986}{24} \frac{1987}{g} \frac{1988}{26} \frac{1989}{g} \frac{1999}{21} \frac{1991}{30} \frac{1992}{g} \frac{1993}{31} \frac{1994}{0}$

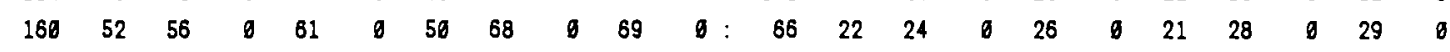

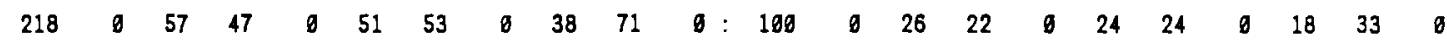

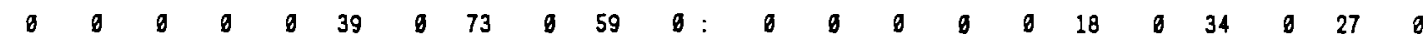

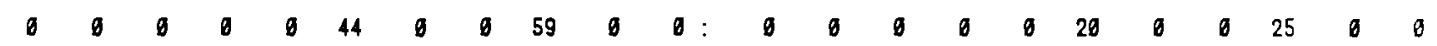

$\begin{array}{llllllllllllllllllllll}0 & 0 & 0 & 0 & 0 & 0 & 0 & 0 & 0 & 0 & 44: & 0 & 0 & 0 & 0 & 0 & 0 & 0 & 0 & 0 & 0 & 20\end{array}$

$\begin{array}{lllllllllllllllllllllll}172 & 17 & 16 & 17 & 0 & 17 & 17 & 29 & 15 & 21 & 16: & 22 & 2 & 2 & 2 & 0 & 2 & 2 & 3 & 2 & 3 & 2\end{array}$

$\begin{array}{lllllllllllllllllllllll}6 & 6 & 6 & 6 & 70 & 53 & 34 & 48 & 38 & 54 & 36: & 0 & 6 & 6 & 0 & 36 & 22 & 14 & 26 & 15 & 23 & 13 \\ & 0 & 0 & 0 & 0 & 42 & 42 & 51 & 34 & 54 & 29 & 0 & 0 & 0 & 0 & 0 & 18 & 18 & 22 & 14 & 23 & 12\end{array}$

$\begin{array}{lrrrrrrrrrrrrrrrrrrrrrr}1688 & 188 & 0 & 197 & 0 & 182 & 0 & 0 & 148 & 0 & 170 & : & 201 & 34 & 0 & 36 & 0 & 33 & 0 & 0 & 27 & 0 & 31\end{array}$

$\begin{array}{llllllllllllllllllllllll}1188 & 0 & 175 & 6 & 0 & 168 & 0 & 267 & 0 & 271 & \theta & : & 222 & 0 & 32 & 0 & 0 & 31 & 0 & 49 & 0 & 49 & 0\end{array}$

$\begin{array}{llllllllllllllllllllll}1904 & 199 & 0 & 237 & 0 & 172 & 0 & 150 & 0 & 173 & 1787 & 0 & 36 & 9 & 43 & 0 & 32 & 0 & 27 & 0 & 31\end{array}$

$\begin{array}{lllllllllllllllllllllll}512 & 0 & 81 & 0 & 135 & 121 & 0 & 167 & 92 & 0 & 110 & : & 96 & 0 & 11 & 0 & 25 & 23 & 0 & 31 & 17 & 0 & 21\end{array}$

$\begin{array}{llllllllllllllllllllll}752 & 141 & 105 & 0 & 121 & 128 & 0 & 94 & 171 & 0 & : & 141 & 0 & 26 & 29 & 0 & 23 & 24 & 0 & 18 & 32 & 0\end{array}$

$\begin{array}{lllllllllllllllllllllll}6 & 0 & 50 & 33 & 48 & 44 & 41 & 49 & 34 & 54 & 30: & 0 & 0 & 21 & 14 & 20 & 19 & 17 & 21 & 14 & 23 & 13\end{array}$

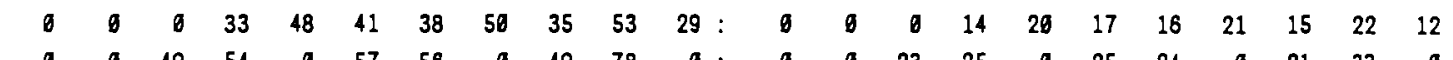

$\begin{array}{rrrrrrrrrrrrrrrrrrrrrr}468 & 87 & 57 & 0 & 71 & 52 & 0 & 70 & 45 & 9 & 47: & 179 & 26 & 22 & 9 & 28 & 26 & 0 & 27 & 18 & 0 & 18\end{array}$

$\begin{array}{llllllllllllllllllllll}391 & 57 & 6 & 51 & 79 & 0 & 55 & 70 & 0 & 70 & 47 & 149 & 22 & 9 & 20 & 31 & 0 & 22 & 27 & 0 & 27 & 18\end{array}$

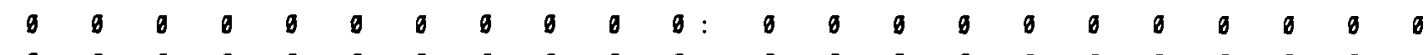

$0 \begin{array}{llllllllllllllllllllll}0 & 0 & 0 & 0 & 0 & 0 & 0 & 0 & 0 & 0: & 0 & 0 & 0 & 0 & 0 & 0 & 0 & 0 & 0 & 0 & 0 \\ 0 & 37 & 0 & 42 & 47 & 45 & 43 & 57 & 30 & 5 & 0 & 0 & 10 & 0 & 18 & 20 & 19 & 10 & 24 & 15 & 21 & 0\end{array}$

$\begin{array}{llllllllllllllllllllll}0 & 37 & 0 & 42 & 47 & 45 & 43 & 57 & 36 & 50 & 0: & 0 & 16 & 0 & 18 & 20 & 19 & 18 & 24 & 15 & 21 & 0\end{array}$

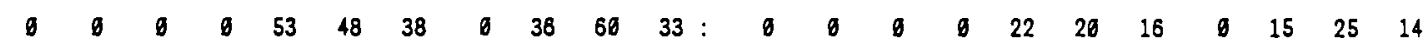

$\begin{array}{lllllllllllllllllllllll}6 & 0 & 0 & 149 & 123 & 0 & 188 & 131 & 0 & 250 & 89 & : & 0 & 0 & 0 & 27 & 23 & 0 & 31 & 24 & 0 & 46 & 15\end{array}$

$\begin{array}{llllllllllllllllllllll}6 & 0 & 0 & 43 & 49 & 47 & 47 & 55 & 46 & 59 & 37: & 0 & 0 & 0 & 20 & 23 & 22 & 22 & 22 & 16 & 24 & 15\end{array}$

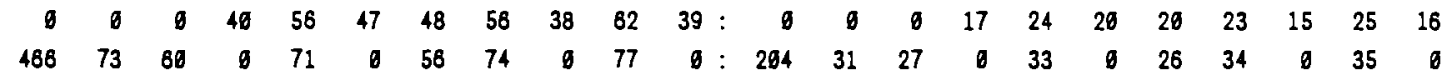

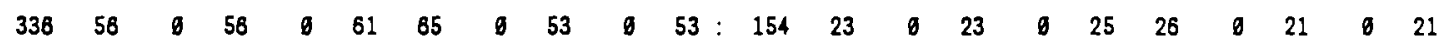

$\begin{array}{llllllllllllllllllllll}914 & 6 & 185 & 63 & 110 & 79 & 84 & 112 & 67 & 113 & 72: & 172 & 0 & 19 & 12 & 20 & 14 & 15 & 28 & 12 & 21 & 13\end{array}$

$\begin{array}{llllllllllllllllllllllll}253 & 17 & 37 & 39 & 0 & 40 & 36 & 9 & 32 & 53 & 0 & : & 118 & 8 & 17 & 18 & 0 & 19 & 17 & 0 & 15 & 25 & 0\end{array}$

$\begin{array}{llllllllllllllllllllllll}197 & 0 & 49 & 47 & 0 & 44 & 48 & 0 & 36 & 68 & 0 & : & 93 & 0 & 23 & 22 & 0 & 21 & 22 & 0 & 17 & 32 & 0\end{array}$

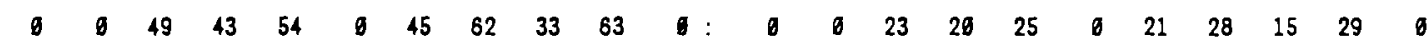

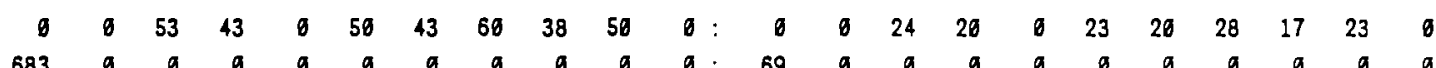

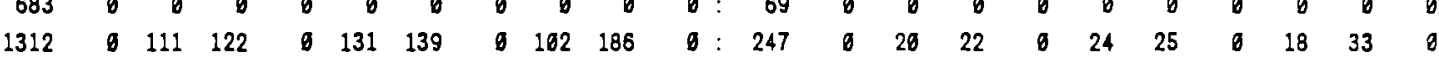

$\begin{array}{lllllllllllllllllllllll}1168 & 92 & 167 & 0 & 143 & 103 & 0 & 146 & 79 & 0 & 97 & 222 & 17 & 28 & 9 & 26 & 19 & 9 & 27 & 15 & 9 & 18\end{array}$

$\begin{array}{lllllllllllllllllllllll}576 & 86 & 0 & 79 & 95 & 9 & 79 & 115 & 0 & 116 & 74 & : & 107 & 16 & 0 & 14 & 17 & 9 & 14 & 21 & 0 & 21 & 14\end{array}$

$\begin{array}{lllllllllllllllllllllll}6 & 0 & 296 & 156 & 0 & 219 & 0 & 256 & 0 & 272 & 0 & 0 & 0 & 0 & 38 & 29 & 0 & 48 & 0 & 47 & 0 & 50 & 0\end{array}$

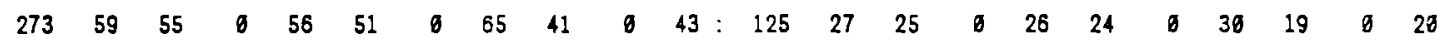

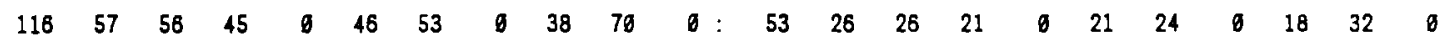

$\begin{array}{llllllllllllllllllllll}816 & 154 & 122 & 0 & 162 & 117 & 0 & 159 & 99 & 0 & 194: & 153 & 28 & 22 & 0 & 30 & 22 & 0 & 29 & 18 & 0 & 19\end{array}$

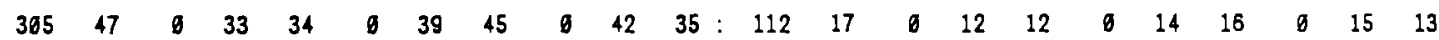

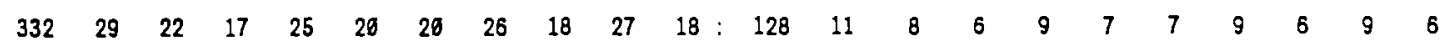

$\begin{array}{lllllllllllllllllllllll}6 & 0 & 199 & 0 & 237 & 174 & 9 & 233 & 141 & 246 & 0 & 0 & 0 & 0 & 37 & 0 & 44 & 32 & 0 & 41 & 25 & 43 & 0\end{array}$

$\begin{array}{rrrrrrrrrrrrrrrrrrrrrrr}0 & 0 & 0 & 0 & 0 & 0 & 0 & 0 & 0 & 263 & 142: & 0 & 0 & 0 & 0 & 0 & 0 & 0 & 0 & 0 & 48 & 25 \\ 545 & 35 & 0 & 34 & 46 & 37 & 0 & 49 & 27 & 0 & 32: & 225 & 14 & 0 & 14 & 19 & 15 & 0 & 28 & 11 & 0 & 13\end{array}$

$\begin{array}{rrrrrrrrrrrrrrrrrrrrrr}545 & 35 & 0 & 34 & 48 & 37 & 0 & 49 & 27 & 0 & 32: & 225 & 14 & 0 & 14 & 19 & 15 & 0 & 28 & 11 & 0 & 13 \\ 0 & 0 & 0 & 0 & 45 & 38 & 0 & 45 & 31 & 0 & 32: & 0 & 0 & 0 & 0 & 21 & 18 & 0 & 21 & 14 & 0 & 15\end{array}$

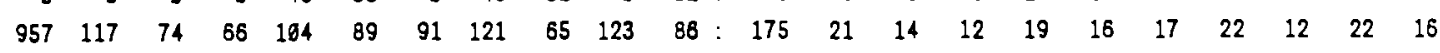


TABLE A.3. Middle Case (with Extended Burnup) 1984 Inventory and Projected Annual Reactor Discharges (contd)

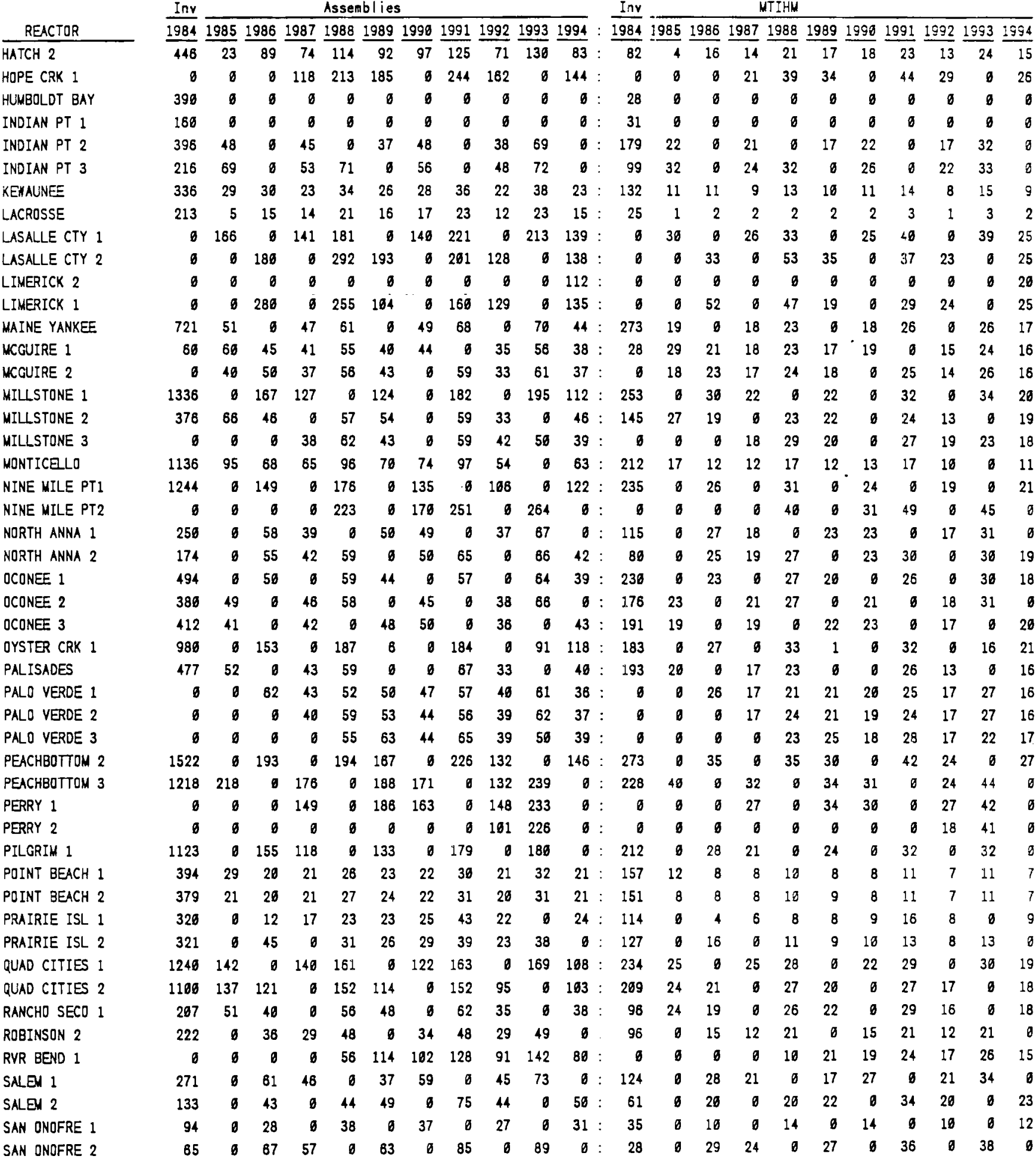


TABLE A.3. Middle Case (with Extended Burnup) 1984 Inventory and Projected Annual Reactor Discharges (contd)

\begin{tabular}{|c|c|c|c|c|c|c|c|c|c|c|c|c|c|c|c|c|c|c|c|c|c|c|}
\hline & Inv & & & & Assen & blies & & & & & & $\operatorname{Inv}$ & & & & MTIHM & & & & & & \\
\hline REACTOR & 1984 & 1985 & 1986 & 1987 & 1988 & 1989 & 1998 & 1991 & 1992 & 1993 & 1994 & $: 1984$ & 1985 & 1986 & 1987 & 1988 & 1989 & 1990 & 1991 & 1992 & 1993 & 1994 \\
\hline SAN ONDFRE 3 & 6 & 46 & 65 & 8 & 78 & $\theta$ & 67 & 9 & 52 & 9 & 57 & 8 & $2 \sigma$ & 28 & 6 & 36 & 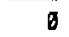 & 29 & 9 & 22 & 9 & 24 \\
\hline SEABROOK 1 & 8 & $\emptyset$ & g & 36 & 64 & 44 & 43 & 56 & 40 & 61 & 34 & $\emptyset$ & $\theta$ & $\varnothing$ & 15 & 27 & 19 & 18 & 24 & 17 & 26 & 14 \\
\hline SEABROOK-2 & 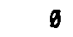 & 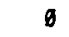 & $\theta$ & g & g & פ & 0 & g & g & 63 & 42 & 0 & $\theta$ & g & 0 & $\theta$ & $\theta$ & $\theta$ & $\theta$ & $\theta$ & 27 & 18 \\
\hline SEQUOYAH 1 & 140 & 42 & $\theta$ & 42 & 63 & $\theta$ & 53 & g & 44 & 77 & 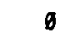 & 64 & 19 & $\theta$ & 19 & 29 & 0 & 24 & $\theta$ & 28 & 35 & $\theta$ \\
\hline SEQUOYAH 2 & 136 & $\theta$ & 48 & 43 & 6 & 51 & 55 & פ & 44 & $\theta$ & 49 & 62 & $\theta$ & 22 & 20 & $\theta$ & 24 & 25 & $\theta$ & 28 & $\theta$ & 22 \\
\hline SHOREHAH & $\theta$ & $g$ & $\theta$ & 142 & 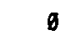 & 144 & 166 & g & 76 & 134 & 0 & 0 & 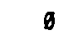 & 0 & 26 & 0 & 26 & 30 & $\theta$ & 14 & 24 & $\theta$ \\
\hline SOUTH TEXAS 1 & $\theta$ & 0 & 8 & 8 & 51 & 49 & 28 & 47 & 28 & 39 & 30 & פ & $\boldsymbol{\theta}$ & $\theta$ & $\theta$ & 27 & 26 & 15 & 25 & 15 & 21 & 16 \\
\hline SOUTH TEXAS 2 & $\theta$ & 9 & $\theta$ & 0 & 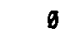 & 6 & $\theta$ & 35 & 28 & 47 & 30 & 0 & 0 & 0 & 0 & $b$ & $\theta$ & $\theta$ & 19 & 15 & 25 & 16 \\
\hline ST LUCIE 1 & 372 & 73 & ø & 47 & 80 & 0 & 55 & 89 & 6 & 77 & 62 & : 142 & 28 & $\theta$ & 17 & 24 & 6 & 20 & 27 & $\theta$ & 29 & 19 \\
\hline ST LUCIE 2 & 80 & $\theta$ & 74 & 54 & $\theta$ & 62 & 57 & 6 & 50 & 78 & 0 & 31 & $\theta$ & 27 & 20 & $\theta^{\circ}$ & 24 & 22 & $\theta$ & 19 & 30 & 6 \\
\hline SUNMER 1 & 44 & 97 & 0 & 52 & 55 & 50 & 47 & 0 & 46 & 62 & 45 & 26 & 45 & ⿹ & 24 & 25 & 23 & 22 & 0 & 18 & 28 & 21 \\
\hline SURRY 1 & 477 & ø & 52 & 38 & 6 & 42 & 44 & 6 & 34 & 57 & $\emptyset$ & 217 & 6 & 24 & 17 & $\theta$ & 19 & 26 & 6 & 16 & 26 & $\theta$ \\
\hline SURRY 2 & 349 & 48 & 45 & 6 & 54 & 43 & $\theta$ & 59 & 31 & 0 & 37 & 158 & 22 & 21 & 6 & 25 & 20 & 0 & 27 & 14 & $\theta$ & 17 \\
\hline SUSQUEHANNA 1 & $\theta$ & 158 & 172 & 156 & 6 & 166 & 167 & 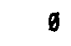 & 131 & 247 & 0 & 6 & 29 & 32 & 29 & 6 & 36 & 29 & $\emptyset$ & 23 & 43 & 6 \\
\hline SUSQUEHANNA 2 & 8 & $\theta$ & 194 & 0 & 223 & 167 & 0 & 216 & 128 & 0 & 145 & 6 & 0 & 36 & $\theta$ & 41 & 31 & 0 & 38 & 22 & 6 & 26 \\
\hline 3 MILE ISL 1 & 268 & $\emptyset$ & $\emptyset$ & 53 & 60 & 6 & 50 & 65 & $\emptyset$ & 71 & 45 & 97 & 0 & 6 & 25 & 28 & 6 & 23 & 30 & $\emptyset$ & 33 & 21 \\
\hline TROJAN & 283 & 28 & 37 & 35 & 41 & 32 & 28 & 37 & 23 & 38 & 25 & $: \quad 130$ & 12 & 17 & 16 & 19 & 15 & 13 & 17 & 11 & 18 & 12 \\
\hline TURKEY PT 3 & 387 & 28 & 36 & 6 & 44 & 39 & 6 & 48 & 36 & 6 & 32 & : 175 & 13 & 16 & 6 & 20 & 18 & 8 & 22 & 14 & $g$ & 15 \\
\hline TURKEY PT 4 & 379 & ø & 6 & 48 & 6 & 34 & 36 & $\theta$ & 36 & $5 \theta$ & g & 172 & $\theta$ & 6 & 22 & $\theta$ & 16 & 17 & 6 & 14 & 23 & $\theta$ \\
\hline VOGRE 1 & 6 & 6 & 6 & 6 & 46 & 6 & 63 & 61 & 38 & 53 & 41 & 6 & 6 & 6 & 6 & 21 & 6 & 29 & 28 & 18 & 24 & 19 \\
\hline VOGRE 2 & $\theta$ & 0 & 6 & 0 & 6 & 6 & 36 & 67 & 38 & 52 & 41 & 6 & 0 & 0 & $\theta$ & 0 & 6 & 17 & 31 & 18 & 24 & 19 \\
\hline VT YANKEE 1 & 1202 & 65 & 0 & 71 & 69 & 61 & 64 & 85 & 48 & 87 & 57 & : 224 & 12 & 6 & 13 & 13 & 11 & 12 & 16 & 9 & 16 & 10 \\
\hline WASH NUCLEAR2 & g & $\theta$ & 152 & 168 & 142 & 104 & 117 & 150 & 85 & 153 & 97 & 6 & 0 & 28 & 20 & 26 & 19 & 21 & 26 & 15 & 27 & 17 \\
\hline WNP-1 & $\theta$ & 6 & 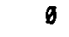 & 0 & 0 & 6 & 6 & $\theta$ & 55 & 149 & 40 & 0 & 6 & $\theta$ & 0 & 0 & 6 & 6 & 0 & 25 & 68 & 18 \\
\hline MNP-3 & 0 & $\theta$ & 6 & 0 & 0 & 6 & 6 & 78 & 43 & 61 & 43 & 6 & 6 & $\emptyset$ & 6 & 6 & 6 & 0 & 33 & 17 & 25 & 19 \\
\hline WATERFORD 3 & $\theta$ & 9 & 59 & 0 & 79 & 47 & 44 & 67 & 6 & 79 & 39 & 6 & 0 & 25 & 6 & 31 & 19 & 19 & 29 & $\theta$ & 30 & 17 \\
\hline WATTS BAR 1 & ø & 27 & 69 & 0 & 52 & $\theta$ & 52 & $\theta$ & 44 & $\theta$ & 43 & 0 & 12 & 31 & 6 & 23 & 6 & 24 & $\theta$ & 20 & $\theta$ & 20 \\
\hline WATTS BAR 2 & 6 & $\theta$ & $\theta$ & 35 & 70 & 6 & 44 & 22 & 35 & $\theta$ & 38 & 8 & $\theta$ & $\theta$ & 16 & 31 & 8 & 26 & 10 & 16 & 0 & 17 \\
\hline WOLF CRK 1 & $\theta$ & $\theta$ & $\theta$ & 30 & 37 & 35 & 31 & 43 & 36 & 45 & 25 & $\theta$ & 6 & $\theta$ & 14 & 17 & 16 & 14 & 26 & 14 & 21 & 12 \\
\hline YANKEE-ROME 1 & 360 & 27 & 0 & 25 & 33 & 6 & 27 & 34 & 22 & 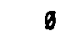 & 22 & 74 & 6 & 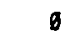 & 6 & 8 & 6 & 6 & 8 & 5 & $\theta$ & 5 \\
\hline ZION 1 & 433 & 27 & 35 & 32 & 42 & 33 & 33 & 45 & 26 & 45 & 29 & $: \quad 197$ & 12 & 16 & 15 & 19 & 15 & 15 & 21 & 12 & 21 & 13 \\
\hline ZION 2 & 422 & 49 & 39 & 31 & 42 & 33 & 34 & 45 & 26 & 46 & 29 & : 192 & 18 & 18 & 14 & 19 & 15 & 16 & 21 & 12 & 21 & 13 \\
\hline BRUNSWICK-1 PWR POOL & $16 \theta$ & & & & & & & & & & & 69 & & & & & & & & & & \\
\hline BRUNSWICK-2 PWR POOL & 144 & & & & & & & & & & & 141 & & & & & & & & & & \\
\hline MORRIS-BWR POOL & 879 & & & & & & & & & & & : 169 & & & & & & & & & & \\
\hline MORRIS-PWR POOL & 350 & & & & & & & & & & & $: \quad 132$ & & & & & & & & & & \\
\hline WEST VALLEY BWR & 316 & & & & & & & & & & & 54 & & & & & & & & & & \\
\hline WEST VALLEY PWR & 121 & & & & & & & & & & & 46 & & & & & & & & & & \\
\hline
\end{tabular}


TABLE A.3. Middle Case (with Extended Burnup) 1984. Inventory and Projected Annual Reactor Discharges (contd)

Assemblies

MTIHM

\begin{tabular}{|c|c|c|c|c|c|c|c|c|c|c|c|c|c|c|c|c|c|c|c|c|}
\hline REACTOR & 995 & 198 & 1997 & 1998 & 1999 & 2000 & $2001 ?$ & 20023 & 2003 & 2004 & 1995 & 19961 & 19971 & 19981 & 19992 & 20002 & 29012 & 20922 & $2003 ?$ & 964 \\
\hline RK NUCLEAR 1 & 55 & $\theta$ & 67 & 41 & $\theta$ & 63 & 8 & 71 & $\theta$ & 54 & 25 & $\theta$ & 31 & 19 & 8 & 29 & $\theta$ & 33 & 9 & 2 \\
\hline RK NUCLEAR 2 & 60 & $\theta$ & 74 & 52 & 6 & 68 & $\theta$ & 75 & 8 & 56 & 25 & $\theta$ & 31 & 22 & $\theta$ & 28 & $\theta$ & 1 & $\theta$ & \\
\hline VALIEY 1 & 60 & 42 & $\theta$ & 45 & 53 & 8 & 37 & 78 & $g$ & 59 & 28 & 19 & 9 & 21 & 24 & $\theta$ & 17 & 36 & $\theta$ & \\
\hline VALLEY 2 & 77 & 6 & 59 & 51 & $\theta$ & 63 & 38 & $\varnothing$ & 49 & 67 & 35 & 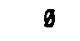 & 27 & 24 & 0 & 29 & 18 & $\theta$ & 18 & \\
\hline BELEONTE 1 & 78 & 38 & $\theta$ & 73 & 53 & 8 & 51 & 90 & 8 & 72 & 36 & 17 & $\theta$ & 33 & 24 & ø & 23 & 41 & $\theta$ & \\
\hline 3ELIEONTE 2 & 80 & 46 & $\theta$ & 52 & 8 & 69 & 51 & $\theta$ & 51 & 85 & 34 & 20 & $g$ & 24 & $g$ & 31 & 23 & $\theta$ & 23 & \\
\hline IG ROCX 1 & $\theta$ & 14 & 21 & 14 & 17 & 18 & 15 & 84 & 8 & ø & $\theta$ & 2 & 3 & 2 & 2 & 2 & 2 & 11 & 8 & \\
\hline RAIDWOOD 1 & 55 & 34 & 48 & 45 & 36 & 49 & 36 & 54 & 30 & 44 & 23 & 14 & 28 & 19 & 15 & 21 & 13 & 23 & 13 & \\
\hline RAIDYODD 2 & 54 & 32 & 48 & 44 & 35 & 48 & 38 & 54 & 39 & 43 & 23 & 14 & 28 & 19 & 15 & 28 & 13 & 23 & 13 & 18 \\
\hline ROWNS FERRY1 & $\theta$ & 156 & 6 & 167 & 9 & 261 & 0 & 289 & $\theta$ & 223 & 8 & 28 & $\theta$ & 30 & $\theta$ & 47 & $\theta$ & 52 & $\oslash$ & \\
\hline ROWNS FERRY2 & 239 & 0 & 290 & 0 & 199 & $\emptyset$ & 162 & $\varnothing$ & 158 & 0 & 42 & $\theta$ & 53 & 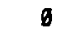 & 36 & 0 & 29 & $\theta$ & 29 & \\
\hline ROWNS FERRY3 & $\emptyset$ & 158 & 6 & 167 & $\emptyset$ & $\emptyset$ & 161 & 8 & 161 & 0 & $\emptyset$ & 29 & $\theta$ & 30 & $\theta$ & $\varnothing$ & 29 & $\theta$ & 29 & \\
\hline UNSWICK 1 & 148 & $\theta$ & 184 & 107 & 6 & 186 & 93 & $\theta$ & 98 & 141 & 28 & $\theta$ & 34 & 29 & 8 & 31 & 17 & 6 & 18 & \\
\hline RUNSWICK 2 & 148 & 161 & 0 & 106 & 127 & 6 & 93 & 183 & 9 & 141 & 28 & 19 & 9 & 20 & 24 & 8 & 17 & 34 & 9 & \\
\hline RON 1 & 54 & 33 & 49 & 45 & $3 S$ & 49 & 29 & 54 & 38 & 44 & 23 & 14 & 21 & 19 & 15 & 21 & 12 & 23 & 13 & \\
\hline RON 2 & 54 & 32 & 47 & 44 & 35 & 48 & 30 & 54 & 30 & 44 & 23 & 14 & 20 & 19 & 15 & 28 & 13 & 23 & 13 & \\
\hline LLAMAY 1 & 69 & 59 & 0 & 52 & 57 & 6 & 51 & 84 & 8 & 87 & 29 & 21 & $\theta$ & 22 & 24 & 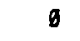 & 22 & 36 & 6 & \\
\hline ALVERT CLF 1 & 58 & $g$ & 77 & 48 & $\varnothing$ & 68 & 56 & 6 & 58 & 59 & 23 & 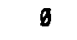 & 36 & 19 & 8 & 27 & 28 & 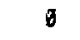 & 20 & \\
\hline LLVERT CLF 2 & $g$ & 48 & 71 & 6 & 53 & 66 & $\theta$ & 76 & 48 & 6 & 9 & 19 & 28 & 8 & 21 & 26 & ø & 30 & 19 & \\
\hline RROLL COUNTY-1 & 8 & 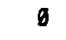 & 9 & $\theta$ & 8 & 6 & 6 & 81 & 31 & 46 & 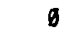 & 9 & $\theta$ & $\theta$ & $\theta$ & 9 & $g$ & 34 & 13 & 15 \\
\hline ARROLL COUNTY-2 & 0 & $g$ & $\theta$ & $\theta$ & $\theta$ & $\emptyset$ & $\theta$ & 54 & 31 & 46 & $\emptyset$ & ø & $\emptyset$ & 6 & 0 & 0 & ø & 23 & 13 & \\
\hline TAMBA 1 & 61 & 35 & 58 & 50 & 37 & 50 & 37 & 54 & 8 & 50 & 26 & 15 & 21 & 21 & 16 & 21 & 16 & 23 & $\emptyset$ & \\
\hline АТАMBA 2 & 60 & 35 & 72 & 41 & 35 & 9 & 37 & 56 & 36 & 44 & 25 & 15 & 30 & 17 & 15 & 9 & 16 & 24 & 15 & 1 \\
\hline INTON 1 & 0 & 159 & 121 & 6 & 155 & 0 & 114 & 194 & $\theta$ & 155 & $\theta$ & 29 & 22 & 6 & 28 & $\theta$ & 21 & 35 & 6 & \\
\hline JMANCHE PK 1 & 62 & 18 & 53 & 51 & 48 & 55 & 41 & 61 & 39 & 50 & 25 & 16 & 21 & 21 & 16 & 22 & 17 & 25 & 16 & 2 \\
\hline DMANCHE PK 2 & 63 & 40 & 53 & 51 & 41 & 54 & 41 & 60 & 39 & 49 & 25 & 16 & 21 & 21 & 17 & 22 & 17 & 24 & 16 & \\
\hline JOK 1 & 66 & 47 & 6 & 54 & 54 & 6 & 48 & 6 & 48 & 65 & 30 & 22 & $\theta$ & 25 & 25 & $\emptyset$ & 22 & 6 & 22 & \\
\hline JOK 2 & 73 & $\theta$ & 89 & 56 & 6 & 172 & $\theta$ & 91 & 56 & 0 & 29 & 0 & 36 & 23 & $g$ & 78 & $\theta$ & 37 & 23 & \\
\hline JOPER STN & 95 & 68 & 116 & 69 & 80 & 195 & 68 & 119 & 68 & 87 & 17 & 12 & 22 & 13 & 15 & 19 & 12 & 22 & 12 & \\
\hline :RYSTAL RVR 3 & 46 & 34 & 6 & 34 & 39 & 6 & 35 & 58 & 0 & 44 & 21 & 16 & $\theta$ & 16 & 18 & $\emptyset$ & 16 & 27 & $\theta$ & \\
\hline AVIS-BESSE 1 & 55 & 39 & 6 & 46 & 46 & 9 & 37 & 71 & $\theta$ & 53 & 26 & 18 & g & 19 & 22 & $\emptyset$ & 17 & 33 & $\emptyset$ & \\
\hline IABLO CNYN 1 & 50 & 39 & 64 & $\theta$ & 44 & 64 & 33 & $\theta$ & 33 & 55 & 23 & 18 & 29 & $\theta$ & 20 & 29 & 15 & 6 & 15 & \\
\hline JEABLO CNYN 2 & 65 & 33 & 69 & $\theta$ & 38 & 59 & 34 & $\theta$ & 32 & 54 & 38 & 15 & 32 & 8 & 17 & 27 & 16 & 6 & 15 & \\
\hline RESDEN 1 & 0 & $\theta$ & $\theta$ & 8 & 0 & 0 & $\theta$ & $\theta$ & $\theta$ & a & 6 & $\theta$ & $\theta$ & $g$ & $\theta$ & $\theta$ & 0 & 8 & 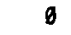 & \\
\hline RESDEN 2 & 158 & 189 & 8 & 115 & 136 & $\emptyset$ & 104 & 199 & 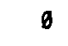 & 153 & 28 & 26 & 6 & 21 & 24 & $\theta$ & 19 & 36 & $\theta$ & \\
\hline DRESDEN 3 & 131 & 6 & 163 & 95 & 8 & 147 & 86 & $\theta$ & 86 & 125 & 24 & 6 & 30 & 17 & $\theta$ & 27 & 16 & 6 & 16 & \\
\hline UANE ARNDLD & 8 & 66 & 123 & 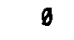 & 84 & 110 & b & 123 & 65 & 0 & $\theta$ & 12 & 22 & $\theta$ & 15 & 20 & 8 & 2.2 & 12 & \\
\hline ENRICO FERMI2 & 276 & 155 & 0 & 225 & $\theta$ & 249 & $\theta$ & 271 & 158 & 6 & 56 & 28 & 0 & 41 & $\theta$ & 45 & 6 & 49 & 29 & \\
\hline ARLEY 1 & 61 & 6 & 72 & 46 & $\theta$ & 63 & 43 & $\theta$ & 39 & 58 & 28 & 6 & 33 & 21 & $\theta$ & 29 & 28 & 8 & 18 & 2 \\
\hline ARLEY 2 & 57 & 45 & 8 & 44 & 53 & 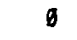 & 39 & 77 & 6 & 54 & 26 & 21 & 6 & 20 & 24 & 6 & 18 & 36 & $g$ & \\
\hline FITZPATRICK & 143 & 0 & 175 & 167 & 0 & 160 & 168 & 6 & 106 & 137 & 26 & 0 & 31 & 19 & $\theta$ & 29 & 19 & 8 & 19 & 2 \\
\hline EORT CALHOUN & 6 & 28 & 41 & 0 & 39 & 45 & 6 & 41 & 25 & 6 & 8 & 10 & 15 & $\theta$ & 14 & 16 & $\theta$ & 1.5 & 9 & \\
\hline GINNA & 23 & 19 & 30 & 19 & 20 & 28 & 19 & 30 & 19 & 23 & 8 & 7 & 11 & 7 & 7 & 9 & 7 & 11 & 7 & \\
\hline GRAND GULF 1 & 218 & 148 & $\theta$ & 151 & 189 & 8 & 149 & 274 & 143 & 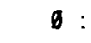 & 38 & 26 & 6 & 27 & 33 & $\theta$ & 26 & 48 & 25 & \\
\hline GRAND GULF 2 & 200 & 129 & 220 & 158 & 143 & 214 & 124 & 219 & 127 & 186 & 37 & 24 & 48 & 29 & 26 & 39 & 23 & 40 & 23 & \\
\hline HADDAM NECX & 42 & 31 & D & 30 & 37 & 47 & $\theta$ & 55 & 28 & $\theta$ & 17 & 13 & 8 & 12 & 15 & 19 & $\emptyset$ & 22 & 12 & \\
\hline HARRIS 1 & 53 & 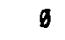 & 44 & 6 & 33 & 52 & 6 & 49 & 33 & 6 & 25 & $\theta$ & 28 & $\theta$ & 15 & 24 & 8 & 23 & 15 & \\
\hline & 04 & 2 & 31 & 82 & 7 & 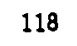 & 64 & 46 & 7 & 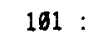 & 9 & 13 & 24 & 15 & 18 & 22 & 12 & 26 & 12 & \\
\hline
\end{tabular}


TABLE A.3. Middle Case (with Extended Burnup) 1984 Inventory and Projected Annual Reactor Discharges (contd)

\begin{tabular}{|c|c|c|c|c|c|c|c|c|c|c|c|c|c|c|c|c|c|c|c|c|c|c|}
\hline \multirow[b]{2}{*}{ REACTOR } & \multirow[b]{2}{*}{$\therefore$} & \multicolumn{11}{|c|}{ Assemblies } & \multicolumn{10}{|c|}{ MTIHN } \\
\hline & & 1995 & 1996 & 1997 & 1998 & 1999 & 2000 & 2001 & 2602 & $\underline{2003}$ & $\underline{2604}$ & & 1995 & $\underline{1996}$ & 1997 & 1998 & 1999 & 2000 & 2901 & 2892 & 2003 & 2004 \\
\hline HATCH 2 & & 112 & 76 & 140 & 81 & 96 & 125 & 68 & 142 & 73 & 108 & & 28 & 14 & 26 & 15 & 18 & 23 & 12 & 26 & 13 & 29 \\
\hline HOPE CRK 1 & & 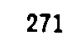 & 9 & 228 & 186 & $\theta$ & 231 & 151 & $\theta$ & 149 & 47 & & 49 & $\theta$ & 41 & 34 & 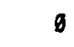 & 42 & 27 & 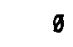 & 27 & 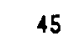 \\
\hline HUMBOLDT BAY & & 6 & 0 & 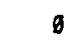 & 6 & 6 & $\theta$ & $\theta$ & $\theta$ & $\theta$ & 6 & • & $\theta$ & $\theta$ & $\theta$ & 6 & $\theta$ & $\theta$ & $\theta$ & 0 & $\theta$ & \\
\hline INDIAN PT 1 & & $\theta$ & 0 & $\theta$ & $\theta$ & $\theta$ & $\theta$ & 0 & $\theta$ & $\theta$ & $\theta$ & & $\theta$ & 0 & $\theta$ & $\theta$ & $\theta$ & $\theta$ & 0 & $\theta$ & $\theta$ & \\
\hline INDIAN PT 2 & & 58 & 41 & $\theta$ & 42 & 51 & $g$ & 41 & 74 & $\theta$ & 57 & : & 27 & 19 & $\theta$ & 19 & 23 & $g$ & 19 & 34 & $\theta$ & 2 \\
\hline INDIAN PT 3 & & 64 & 6 & 79 & 55 & 8 & 68 & 0 & 89 & 43 & 8 & & 29 & $\theta$ & 36 & 25 & $\theta$ & 31 & $\theta$ & 41 & 28 & \\
\hline KEYAUNEE & & 32 & 22 & 41 & 24 & 27 & 33 & 23 & 41 & 23 & 30 & & 12 & 8 & 16 & 9 & 10 & 13 & 9 & 16 & 9 & 12 \\
\hline LACROSSE & & 26 & 13 & 25 & 14 & 17 & 23 & 11 & 24 & 13 & 72 & : & 2 & 1 & 3 & 2 & 2 & 3 & 1 & 3 & 1 & \\
\hline LASALLE CTY 1 & & 6 & 126 & 230 & $\theta$ & 156 & 207 & 0 & 238 & 129 & $\theta$ & : & $\varnothing$ & 23 & 42 & $\theta$ & 28 & 38 & $\theta$ & 42 & 23 & \\
\hline LASALLE CTY 2 & & 190 & $\theta$ & 230 & 133 & $\theta$ & 268 & 132 & $\theta$ & 129 & 176 & & 34 & $\theta$ & 43 & 24 & $\theta$ & 38 & 24 & $\emptyset$ & 23 & 3 \\
\hline LIMERICK 2 & & 6 & 253 & 155 & $\theta$ & 272 & 244 & $g$ & 155 & 193 & 9 & & $g$ & 48 & 28 & $\theta$ & 59 & 44 & 9 & 28 & 35 & \\
\hline LIMERICK 1 & - & 219 & 6 & 186 & 152 & $\theta$ & 188 & 143 & $\theta$ & 137 & 202 & : & 40 & $\theta$ & 34 & 28 & $\theta$ & 34 & 26 & $\theta$ & 25 & 3 \\
\hline MAINE YANKEE & & 6 & 42 & 74 & $\theta$ & 51 & 66 & $\theta$ & 74 & 43 & 0 & : & $\theta$ & 16 & 28 & $\theta$ & 19 & 25 & $\theta$ & 28 & 16 & \\
\hline MCGUIRE 1 & & 52 & 35 & 62 & 39 & 42 & 9 & 36 & 65 & 36 & 46 & & 22 & 15 & 26 & 17 & 18 & 9 & 15 & 28 & 15 & 1 \\
\hline MCCUIRE 2 & & 54 & 34 & 65 & 6 & 44 & 57 & 37 & 62 & 36 & 47 & & 23 & 14 & 28 & $\theta$ & 19 & 24 & 16 & 26 & 15 & 2 \\
\hline MILSTONE 1 & & $\emptyset$ & 110 & $\theta$ & 121 & $\theta$ & 168 & 0 & 268 & 107 & $g$ & : & $\theta$ & 28 & $g$ & 21 & $g$ & 30 & 0 & 37 & 19 & \\
\hline MILSTONE 2 & & 51 & 0 & 67 & 49 & 9 & 58 & 35 & $\theta$ & 38 & 61 & : & 21 & 0 & 27 & 29 & 0 & 24 & 14 & $\theta$ & 15 & 2 \\
\hline MILLSTONE 3 & & $\theta$ & 39 & 51 & 52 & 37 & 65 & 37 & $\theta$ & 39 & 43 & : & $g$ & 18 & 24 & 24 & 17 & 39 & 17 & 0 & 18 & 2 \\
\hline MONTICELO & & 85 & 58 & 92 & 0 & 95 & 83 & 59 & 167 & 59 & 82 & : & 15 & $10^{\circ}$ & 16 & $\theta$ & 17 & 15 & 10 & 19 & 10 & 1 \\
\hline NINE MILE PTI & & 0 & 114 & 6 & 117 & 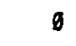 & 184 & $\theta$ & 207 & $\sigma$ & 158 & : & $\varnothing$ & 28 & 6 & 21 & $\theta$ & 32 & 6 & 36 & 0 & 2 \\
\hline NINE MILE PT2 & & 249 & $\emptyset$ & 224 & 184 & 6 & 227 & $\emptyset$ & 250 & $g$ & 295 & : & 46 & $\theta$ & 40 & 33 & $\theta$ & 41 & $\theta$ & 45 & $\theta$ & 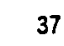 \\
\hline NORTH ANNA 1 & & 57 & 38 & $\theta$ & 42 & 49 & $\theta$ & 39 & 72 & $\theta$ & 54 & : & 26 & 18 & $\theta$ & 19 & 23 & $\theta$ & 18 & 33 & 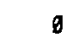 & 2 \\
\hline NORTH ANNA 2 & & 0 & 39 & 71 & $\theta$ & 48 & 62 & 0 & 73 & 38 & $\theta$ & : & $\theta$ & 18 & 33 & 6 & 22 & 29 & $\theta$ & 34 & 18 & \\
\hline OCONEE 1 & & $\emptyset$ & 38 & 68 & 6 & 45 & $\theta$ & 38 & 85 & $\theta$ & 52 & : & $\theta$ & 18 & 32 & 6 & 21 & $\theta$ & 18 & 30 & 6 & 2 \\
\hline OCONEE 2 & & 57 & 39 & $\theta$ & 40 & $\theta$ & 66 & $\theta$ & 67 & 38 & $\theta$ & : & 26 & 18 & $\theta$ & 19 & $\theta$ & 31 & 9 & 31 & 18 & \\
\hline OCONEE 3 & & 56 & 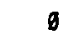 & 72 & 6 & 47 & $g$ & 38 & 72 & $\theta$ & 54 & : & 26 & $\emptyset$ & 33 & $\theta$ & 22 & $\theta$ & 18 & 33 & 6 & 2 \\
\hline OYSTER CRK 1 & & $\theta$ & 41 & 0 & 97 & $\emptyset$ & 156 & 106 & $\theta$ & 98 & 6 & : & 6 & 7 & $\theta$ & 17 & $\theta$ & 28 & 19 & 0 & 17 & \\
\hline PALISADES & & 64 & 34 & $\theta$ & 41 & 45 & 0 & 34 & 66 & 6 & 53 & : & 25 & 13 & 6 & 16 & 18 & $\theta$ & 13 & 26 & 9 & 2. \\
\hline PALD VERDE 1 & & 62 & 39 & 55 & 52 & 41 & 56 & 39 & 62 & 38 & 50 & : & 27 & 17 & 24 & 23 & 18 & 24 & 17 & 27 & 17 & 2 \\
\hline PALO VERDE 2 & & 62 & 39 & 55 & 52 & 41 & 56 & 39 & 61 & 38 & 56 & : & 27 & 17 & 24 & 23 & 18 & 24 & 17 & 27 & 17 & 2 \\
\hline PALO VERDE 3 & & 6 & 37 & 70 & 44 & 40 & 66 & 39 & 59 & 38 & 59 & : & 27 & 16 & 31 & 19 & 17 & 29 & 17 & 26 & 17 & 26 \\
\hline PEACHBOTTOH 2 & & 200 & 0 & 247 & 144 & 0 & 220 & 143 & $\emptyset$ & 145 & 188 & : & 37 & $\emptyset$ & 46 & 27 & $\theta$ & 41 & 26 & $\theta$ & 27 & 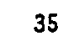 \\
\hline PEACHBOTTOM 3 & & 296 & 143 & $\theta$ & 148 & 175 & $\emptyset$ & 142 & 258 & $\theta$ & 190 & : & 38 & 26 & $\theta$ & 27 & 32 & $\theta$ & 26 & 47 & D & 35 \\
\hline PERRY 1 & & 235 & 143 & $\theta$ & 191 & 154 & $\theta$ & 136 & 232 & 0 & 187 & : & 43 & 26 & 0 & 35 & 28 & $\theta$ & 25 & 42 & $\theta$ & 34 \\
\hline PERRY 2 & & 365 & 173 & $\theta$ & 217 & 195 & 0 & 159 & 298 & 0 & 251 & : & 56 & 32 & $\theta$ & 40 & 36 & 0 & 29 & 54 & $\boldsymbol{\theta}$ & 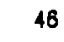 \\
\hline PILGRIM 1 & & 157 & 186 & $\theta$ & 115 & $\emptyset$ & 180 & $g$ & 195 & $\emptyset$ & 154 & : & & 19 & $\emptyset$ & 20 & छ & 32 & $b$ & 5 & 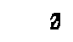 & 27 \\
\hline POINT BEACH 1 & & & 21 & 34 & & 22 & 28 & 2 & 35 & 22 & 26 & : & & 7 & 2 & 8 & 8 & 10 & 8 & & 8 & \\
\hline POINT BEACH 2 & & 2 & 22 & 33 & 22 & 23 & 30 & 2 & 34 & 22 & 26 & & 1 & 8 & 12 & 8 & 8 & 21 & 8 & 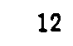 & 8 & \\
\hline PRAIRIE ISL 1 & & 32 & 25 & 41 & 25 & 0 & 37 & 25 & 41 & 25 & 31 & & 12 & 9 & 15 & 9 & $\theta$ & 14 & 9 & 15 & 9 & 11 \\
\hline PRAIRIE ISL 2 & & 33 & 25 & 41 & 25 & 28 & 38 & 0 & 41 & 25 & 31 & : & 11 & 8 & 14 & 8 & 9 & 13 & $\theta$ & 14 & 8 & 10 \\
\hline QUAD CITIES 1 & & $\theta$ & 180 & 182 & g & 123 & 163 & $\theta$ & 180 & 101 & $\theta$ & - & $B$ & 18 & 32 & a & 22 & 29 & 6 & 32 & 18 & \\
\hline QUAD CITIES 2 & & 135 & $\theta$ & 173 & 162 & $\theta$ & 156 & 104 & 6 & 192 & 132 & & 24 & 0 & 30 & 18 & 0 & 27 & 18 & $\theta$ & 18 & 23 \\
\hline RANCHO SECO 1 & & 50 & 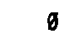 & 61 & 37 & 6 & 55 & 37 & 0 & 37 & 48 & & 23 & 0 & 28 & 17 & 0 & 25 & 17 & $b$ & 17 & 22 \\
\hline ROBINSON 2 & & 42 & 30 & 54 & 31 & $\emptyset$ & 46 & 31 & 53 & 30 & 6 & $\cdot$ & 18 & 13 & 23 & 13 & $\theta$ & 21 & 13 & 23 & 13 & \\
\hline RVR BEND 1 & & 144 & 89 & 128 & 119 & 93 & 131 & 84 & $143^{\circ}$ & 82 & 114 & : & 26 & 16 & 23 & 22 & 17 & 24 & 15 & 26 & 15 & 21 \\
\hline SALEN 1 & & 61 & 47 & $\theta$ & 47 & 53 & 6 & 47 & 78 & 6 & $6 \theta$ & & 28 & 22 & 0 & 22 & 24 & 0 & 22 & 36 & $\theta$ & 28 \\
\hline SALEN 2 & & 63 & $\emptyset$ & 77 & 47 & $\sigma$ & 71 & 47 & $\theta$ & 47 & 59 & & 29 & 6 & 35 & 22 & $\theta$ & 33 & 22 & $g$ & 22 & 27 \\
\hline SAN ONOFRE 1 & & 0 & 29 & $\theta$ & 33 & 46 & 157 & $\theta$ & 6 & 6 & 0 & & $\theta$ & 11 & 0 & 12 & 17 & 58 & $\theta$ & D & $\theta$ & \\
\hline
\end{tabular}


TABLE A.3. Middle Case (with Extended Burnup) 198.1 Inventory and Projected Annual Reactor Discharges (contd)

Assemblies

NTIHM

\begin{tabular}{|c|c|c|c|c|c|c|c|c|c|c|c|c|c|c|c|c|c|c|c|c|}
\hline REACTOR & 995 & 1996 & 1997 & 1998 & 1999 & 2860 & 2061 & 2062 & $\underline{2063}$ & 2064 & 1995 & $\underline{1996}$ & $\underline{1997}$ & 1998 & 1999 & 2000 & $\underline{2601}$ & $\underline{2602}$ & $\underline{2003}$ & 2004 \\
\hline SAN ONOFRE 2 & 74 & g & 98 & g & 63 & $\theta$ & 58 & g & 59 & 78 & 32 & 8 & 38 & $\theta$ & 27 & 8 & 25 & $\theta$ & 25 & 38 \\
\hline SAN ONOFRE 3 & $g$ & 57 & 93 & $g$ & 66 & $\theta$ & 58 & $\theta$ & 59 & 0 & $\theta$ & 24 & 48 & 6 & 28 & 8 & 25 & $\theta$ & 25 & \\
\hline SEABROOK 1 & 62 & 37 & 54 & 51 & 40 & 55 & 33 & 60 & 34 & 49 & 26 & 16 & 23 & 22 & 17 & 23 & 14 & 25 & 14 & 21 \\
\hline SEABROOK-2 & 62 & 37 & 67 & 47 & 42 & 64 & 36 & 65 & 37 & 56 & 26 & 16 & 28 & 20 & 18 & 27 & 15 & 28 & 16 & 24 \\
\hline SERUDYAH 1 & 84 & 47 & $\theta$ & 49 & $g$ & 73 & 48 & $\theta$ & 49 & 62 & 29 & 22 & $\theta$ & 22 & $\theta$ & 34 & 22 & $\theta$ & 22 & 28 \\
\hline SEQUDYAH 2 & 85 & $\theta$ & 82 & $\theta$ & 57 & 74 & D & 81 & 48 & $\theta$ & 30 & $\theta$ & 38 & $\theta$ & 26 & 34 & 6 & 37 & 22 & \\
\hline SHOREHAN & 99 & 85 & $\emptyset$ & $\emptyset$ & 84 & $\theta$ & 84 & 8 & $\theta$ & 132 & 18 & 15 & $\emptyset$ & 0 & 15 & 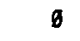 & 15 & 1 & 8 & 24 \\
\hline SOUTH TEXAS 1 & 48 & 26 & 54 & 34 & 27 & 49 & 26 & 45 & 26 & 43 & 26 & 14 & 29 & 18 & 15 & 26 & 14 & 24 & 14 & 23 \\
\hline SOUTH TEXAS 2 & 48 & 28 & 48 & 37 & 31 & 48 & 29 & 48 & 28 & 42 & 26 & 15 & 26 & 20 & 17 & 26 & 16 & 26 & 15 & 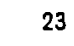 \\
\hline LUCIE 1 & 69 & $g$ & 96 & 52 & $g$ & 81 & 52 & $\theta$ & 64 & 63 & 28 & $\theta$ & 36 & 19 & $\theta$ & 25 & 19 & $g$ & 28 & 23 \\
\hline ST LUCIE 2 & 68 & 52 & $g$ & 53 & 58 & 0 & 52 & 84 & $\theta$ & 83 & 25 & 20 & 0 & 20 & 22 & 0 & 26 & 32 & 0 & 24 \\
\hline SUMMER 1 & 52 & $\theta$ & 75 & 38 & 51 & 58 & $\emptyset$ & 78 & 34 & 56 & 24 & 6 & 35 & 17 & 23 & 27 & 6 & 35 & 16 & 26 \\
\hline SURRY 1 & 51 & 35 & ס & 37 & 41 & o & 36 & 63 & $\theta$ & 48 & 23 & 16 & 6 & 17 & 19 & 6 & 17 & 29 & $\theta$ & 22 \\
\hline JRRY 2 & 51 & $\theta$ & 64 & 35 & 0 & 57 & 35 & 0 & 36 & 46 & 23 & 6 & 29 & 16 & 6 & 26 & 16 & 8 & 17 & 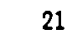 \\
\hline SUSQUEHANNA 1 & 179 & 130 & $\theta$ & 141 & 187 & 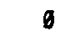 & 138 & 245 & $\emptyset$ & 188 & 31 & 23 & 6 & 25 & 29 & 6 & 24 & 43 & $\theta$ & 33 \\
\hline SUSQUETANNA 2 & 197 & $\theta$ & 244 & 142 & $\emptyset$ & 222 & 137 & D & 134 & 190 & 35 & $\theta$ & 43 & 25 & $\theta$ & 39 & 24 & 6 & 24 & 3 \\
\hline 3 MILE ISL 1 & 0 & 42 & 76 & 0 & 52 & 68 & 0 & 76 & 41 & 0 & 6 & 19 & 35 & 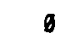 & 24 & 32 & $\theta$ & 35 & 19 & 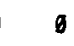 \\
\hline TROJAN & 32 & 24 & 42 & 24 & 28 & 36 & 25 & 42 & 24 & 32 & 15 & 11 & 19 & 11 & 13 & 17 & 12 & 19 & 11 & 15 \\
\hline TURKEY PT 3 & 43 & $\theta$ & 53 & 33 & 8 & 46 & 33 & $g$ & 33 & 41 & 26 & 6 & 24 & 15 & $\theta$ & 21 & 15 & 8 & 15 & 19 \\
\hline TURKEY PT 4 & 44 & 32 & 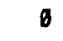 & 33 & 37 & 9 & 33 & 53 & 9 & 41 & 29 & 15 & 0 & 15 & 17 & 6 & 15 & 24 & $\theta$ & 19 \\
\hline VOGTLE 1 & 65 & 34 & 72 & 46 & 37 & 67 & 35 & 68 & 34 & 59 & 30 & 16 & 33 & 21 & 17 & 31 & 16 & 28 & 16 & 27 \\
\hline VDGRE 2 & 63 & 34 & 72 & 45 & 36 & 65 & 35 & 59 & 34 & 58 & 29 & 16 & 33 & 21 & 17 & 36 & 16 & 27 & 16 & 27 \\
\hline VT YANKEE 1 & 75 & 51 & 95 & 53 & 64 & 83 & 58 & 94 & 50 & 71 & 14 & 9 & 17 & 10 & 12 & 15 & 9 & 17 & 9 & 13 \\
\hline WASH NUCLEAR2 & 132 & 98 & 167 & 96 & 112 & 147 & 93 & 165 & 91 & 128 & 23 & 16 & 29 & 17 & 20 & 26 & 16 & 29 & 16 & 23 \\
\hline WP-1 & 63 & 38 & 67 & 47 & 42 & 64 & 36 & 65 & 37 & 55 & 29 & 17 & 31 & 21 & 19 & 29 & 16 & 36 & 17 & 25 \\
\hline WNP-3 & 69 & 41 & 73 & 51 & 47 & 70 & 35 & 70 & 40 & 59 & 30 & 18 & 32 & 22 & 26 & 36 & 15 & 36 & 17 & 25 \\
\hline ATERFORD 3 & 76 & 34 & 6 & 61 & 36 & 72 & 35 & 6 & 49 & 58 & 36 & 15 & 6 & 26 & 15 & 31 & 15 & 0 & 21 & 25 \\
\hline WATS BAR 1 & 75 & $\theta$ & 68 & 54 & 6 & 68 & 0 & 77 & 47 & 0 & 34 & 0 & 31 & 25 & 6 & 31 & 6 & 35 & 22 & ? \\
\hline WATTS BAR 2 & 6 & 46 & 87 & $\theta$ & 49 & 81 & $\theta$ & 73 & 6 & 61 & $\theta$ & 21 & 46 & 6 & 22 & 37 & 6 & 34 & $\theta$ & 28 \\
\hline WOLF CRK 1 & 46 & 29 & 41 & 38 & 31 & 46 & 26 & 45 & 26 & 36 & 21 & 13 & 19 & 18 & 14 & 18 & 12 & 21 & 12 & 17 \\
\hline YANKEE-ROHE 1 & 34 & $\theta$ & 43 & 76 & 6 & 6 & 6 & 6 & 6 & 6 & 8 & 8 & 10 & 18 & $\theta$ & $\theta$ & $\theta$ & $\theta$ & $\theta$ & 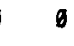 \\
\hline ZION 1 & 40 & 27 & 49 & 29 & 33 & 46 & 28 & 51 & 27 & 38 & 18 & 12 & 22 & 13 & 15 & 21 & 13 & 23 & 12 & 17 \\
\hline ZION 2 & 40 & 27 & 56 & 29 & 34 & 44 & 28 & 51 & 27 & 38 & 18 & 12 & 23 & 13 & 16 & 28 & 13 & 23 & 12 & 17 \\
\hline GENERIC PWR & $\emptyset$ & 6 & $\theta$ & 6 & 6 & 6 & 6 & 6 & 124 & 442 & 8 & $\theta$ & 8 & 6 & $\theta$ & $\theta$ & 6 & $\theta$ & 57 & 284 \\
\hline GENERIC BWR & 6 & 6 & 6 & 6 & 8 & $\theta$ & 6 & $\theta$ & 285 & 675 & $\theta$ & $\theta$ & $\theta$ & $\theta$ & $\theta$ & $\theta$ & $\theta$ & $\theta$ & 52 & \\
\hline TOTAL GEYERIC & 6 & 6 & $\emptyset$ & 6 & $\emptyset$ & $\emptyset$ & 6 & $\theta$ & 499 & 1117 & $\theta$ & $g$ & $\theta$ & 8 & 8 & 8 & 0 & $\theta$ & 109 & \\
\hline
\end{tabular}


TABLE A.3. Middle Case (with Extended Burnup) 1984 Inventory and Projected Annual Reactor Discharges (contd)

Assemblies

MTIHM

REACTOR $\underline{2005} 2006$ 2007 2008 2009 201020112012 2013 2014 : 2005 2006 2007 2008 2009 2010 2011 2012 2013 2004

ARK NUCLEAR 2

Q YALLEY 1

Q VALLEY 2

BELEONTE 1

BELEFONTE 2

BIG ROCX 1

BRAIDYOOD 1

BRAIDYOOD 2

BROWNS FERRY1

BROWNS FERRY2

BROWNS FERRY3

BRUNSWICK 1

BRUNSHICK 2

BYRON 1

BYRON 2

CALLAIIAY 1

CALYERT CLF 1

CALYERT CLF 2

CARROLL COUNTY-1

CARROLL COUNTY-2

CATAWBA 1

CATAMBA 2

CLINTON 1

COMANCHE PK 1

COMANCHE PK 2

COOK 1

COOK 2

COOPER STN

CRYSTAL RVR 3

DAYIS-BESSE 1

DIABLO CNYN 1

DIABLO CNYN 2

DRESDEN 1

DRESDEN 2

DRESDEN 3

DUANE ARNOLD

ENRICO FERMI2

FARLEY 1

FARLEY 2

FITZPATRICK

FORT CALHOUN

GINNA

GRAND GULF 1

GRAND GULF 2

HADDAM NECK

HARRIS 1

HATCH 1

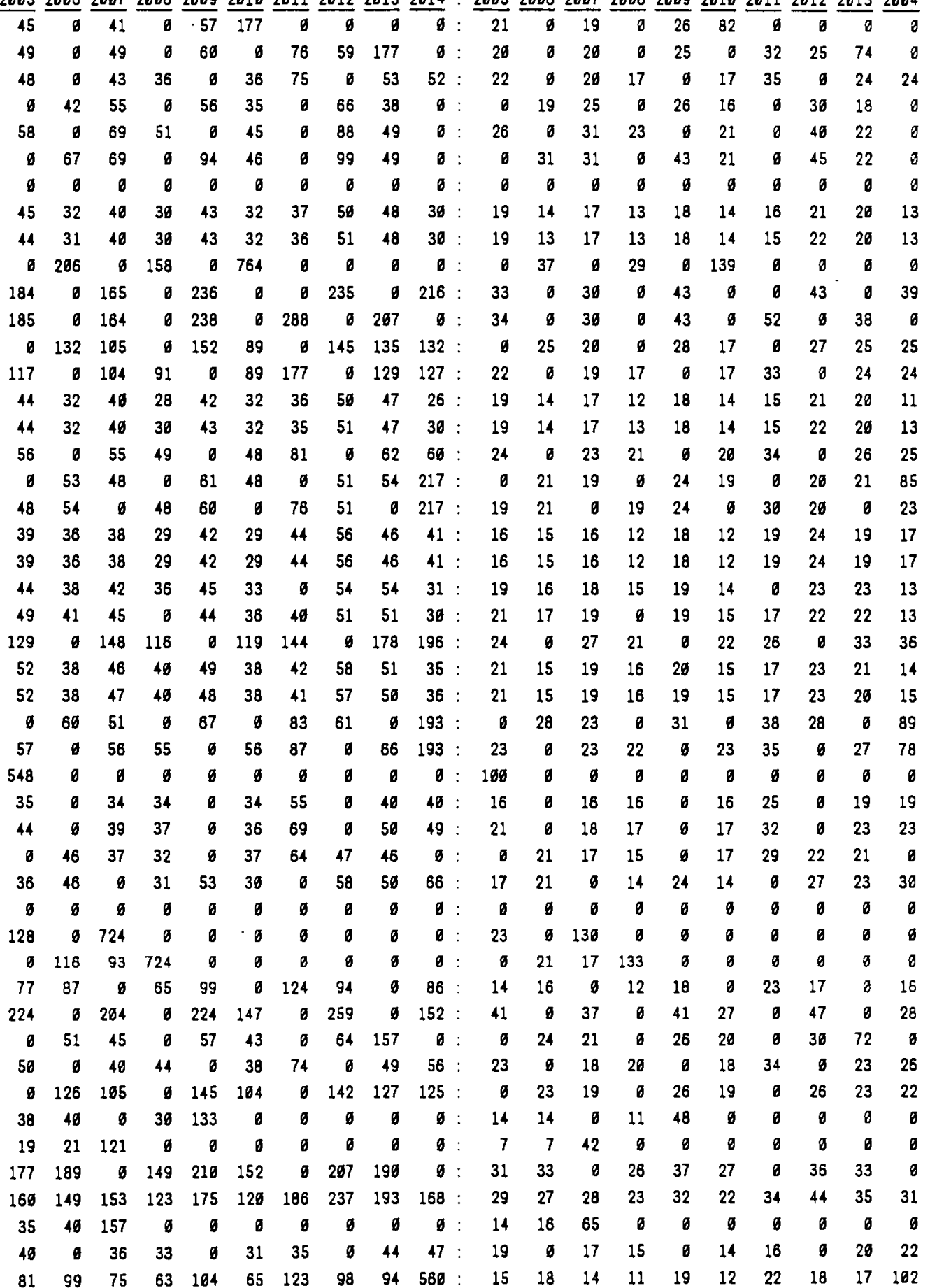


TABLE A.3. Middle Case (with Extended Burnup) 1984 Inventory and Projected Annual Reactor Discharges (contd)

Assemblies

MTIHY

\begin{tabular}{|c|c|c|c|c|c|c|c|c|c|c|c|c|c|c|c|c|c|c|c|c|}
\hline REACTOR & 0065 & 2006 & 2007 & 2008 & 2089 & 2010 & 2011 & 2012 & 2013 & 2614 & 2605 & 0066 & 2007 & 2008 & 2069 & 2010 & 2011 & 2012 & 2013 & 2004 \\
\hline HATCH 2 & 89 & 100 & 78 & 67 & 113 & 65 & 139 & 106 & 99 & 560 & 16 & 18 & 14 & 12 & 21 & 12 & 25 & 19 & 18 & 132 \\
\hline HOPE CRK 1 & 0 & 154 & 200 & $\sigma$ & 216 & 138 & 6 & 243 & 141 & 6 & 6 & 28 & 36 & 0 & 38 & 25 & $\theta$ & 44 & 26 & \\
\hline HUMBOLDT BAY & $\theta$ & 0 & 0 & 6 & 0 & 6 & 6 & $\theta$ & $\theta$ & 0 & 6 & 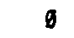 & $\theta$ & $\theta$ & $\theta$ & $\theta$ & $g$ & $\theta$ & 6 & \\
\hline INDIAN PT 1 & $\theta$ & 0 & $\theta$ & $\theta$ & 0 & 6 & 9 & 6 & $\theta$ & $\theta$ & $\theta$ & $\theta$ & $\theta$ & $\theta$ & $\theta$ & $g$ & $\theta$ & $\theta$ & $a$ & \\
\hline INDIAN PT 2 & 48 & $\emptyset$ & 42 & 41 & 6 & 193 & 6 & $\theta$ & 6 & 6 & 22 & 6 & 19 & 19 & $g$ & 89 & $\theta$ & $\theta$ & $\theta$ & \\
\hline INDIAN PT 3 & 55 & $\theta$ & 55 & 56 & 0 & 49 & 6 & 59 & 54 & 0 & 25 & $\theta$ & 25 & 23 & $\theta$ & 22 & $\theta$ & 27 & 25 & \\
\hline KENAUNEE & 25 & 28 & 22 & 23 & 31 & 121 & $\emptyset$ & 6 & 6 & 6 & 16 & 11 & 8 & 9 & 12 & 46 & $\theta$ & $\theta$ & 0 & \\
\hline LACROSSE & 0 & 6 & 6 & 6 & 6 & $\theta$ & 6 & 6 & $\theta$ & $\theta$ & 6 & 6 & $\theta$ & 0 & $\theta$ & $g$ & $\theta$ & $\theta$ & $\theta$ & \\
\hline LASALLE CTY 1 & 146 & 163 & $\theta$ & 129 & 186 & 6 & 232 & 176 & 9 & 159 & 27 & 39 & $\theta$ & 23 & 34 & $\theta$ & 42 & 32 & $\emptyset$ & 29 \\
\hline LASALLE CTY 2 & $\theta$ & 163 & 132 & 6 & 187 & 127 & 6 & 179 & 161 & 6 & 6 & 30 & 24 & $\theta$ & 34 & 23 & $\theta$ & 33 & 29 & \\
\hline LIMERICK 2 & 176 & 154 & 6 & 207 & 219 & $\theta$ & 291 & 219 & 6 & 180 & 32 & 28 & $\theta$ & 38 & 40 & $\theta$ & 53 & 40 & 6 & 33 \\
\hline LIMERICK 1 & $\theta$ & 145 & 164 & $\theta$ & 168 & 138 & $\theta$ & 295 & 199 & 6 & $g$ & 26 & 30 & $\theta$ & 31 & 25 & 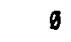 & 37 & 20 & \\
\hline MAINE YANKEE & 46 & 52 & 6 & 217 & $\theta$ & $\emptyset$ & 6 & 6 & 6 & 6 & 17 & 29 & g & 81 & $\theta$ & 0 & $\theta$ & 6 & 6 & \\
\hline MCGUIRE 1 & 43 & 44 & 38 & 35 & 0 & 34 & 66 & 46 & 43 & 44 & 18 & 19 & 16 & 15 & $\theta$ & 14 & 28 & 19 & 18 & 19 \\
\hline MCGUIRE 2 & 41 & 44 & $g$ & 35 & 53 & 33 & 66 & 46 & 47 & 44 & 17 & 19 & $g$ & 15 & 22 & 14 & 28 & 19 & 20 & 19 \\
\hline MILLSTONE 1 & 117 & 6 & 588 & $\theta$ & $\theta$ & 0 & $\theta$ & 6 & $\theta$ & 0 & 21 & $g$ & 163 & $g$ & 0 & 0 & 6 & 6 & $\theta$ & \\
\hline MILLSTONE 2 & 0 & 45 & 39 & 0 & 58 & 40 & 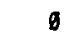 & 51 & 46 & 52 & $g$ & 18 & 18 & $\theta$ & 24 & 16 & $\theta$ & 21 & 19 & 21 \\
\hline MILLSTONE 3 & 52 & 37 & 45 & 37 & 61 & 35 & $g$ & 58 & 54 & 33 & 24 & 17 & 21 & 17 & 28 & 16 & 6 & 27 & 25 & 15 \\
\hline MONTICE—O & 67 & 76 & 484 & $\emptyset$ & $\theta$ & $\theta$ & 0 & $g$ & $\theta$ & $\theta$ & 12 & 13 & 86 & $\theta$ & $\theta$ & 6 & 6 & $\emptyset$ & $\theta$ & \\
\hline NINE MILE PTI & $\emptyset$ & 148 & 532 & $\emptyset$ & $\emptyset$ & $g$ & 9 & $\theta$ & 0 & $\emptyset$ & 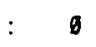 & 26 & 94 & $\theta$ & 0 & 0 & $\theta$ & 9 & $\theta$ & \\
\hline NINE KILE PT2 & 162 & 9 & 188 & $\theta$ & 206 & $\theta$ & 168 & 265 & $\theta$ & 139 & 29 & $\theta$ & 34 & $\theta$ & 37 & $\theta$ & 30 & 48 & $\theta$ & 20 \\
\hline NORTH ANNA 1 & 45 & $\varnothing$ & 41 & 38 & $\theta$ & 38 & 72 & 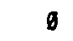 & 62 & 51 & 21 & 0 & 19 & 18 & 6 & 18 & 33 & 9 & 29 & 24 \\
\hline NORTH ANNA 2 & 45 & 49 & $\emptyset$ & 38 & 58 & פ & 72 & 73 & $g$ & 56 & 21 & 23 & $\theta$ & 18 & 27 & 6 & 33 & 34 & $\theta$ & 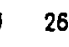 \\
\hline OCONEE 1 & $\theta$ & 48 & 39 & $\theta$ & 177 & 9 & 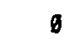 & $g$ & $g$ & $\theta$ & 0 & 22 & 18 & $\theta$ & 82 & 6 & 6 & g & $\theta$ & \\
\hline OCONEE 2 & 46 & $\theta$ & 40 & 37 & 0 & 177 & 0 & $g$ & 0 & $\theta$ & 21 & 0 & 19 & 17 & 0 & 82 & $B$ & 0 & 0 & \\
\hline OCONEE 3 & 45 & 9 & 40 & 0 & 58 & 177 & 0 & $\theta$ & 0 & $\theta$ & 21 & 0 & 19 & 0 & 27 & 82 & $\theta$ & $\theta$ & $\theta$ & \\
\hline OYSTER CRK 1 & 124 & $\theta$ & 568 & $\theta$ & 0 & 6 & $\theta$ & $\theta$ & $\theta$ & $\theta$ & 22 & 0 & 99 & $\theta$ & 6 & 6 & $\emptyset$ & $\theta$ & 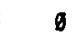 & \\
\hline PALISADES & 40 & $\theta$ & 49 & 204 & 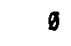 & $b$ & 0 & 0 & 0 & $\theta$ & 16 & 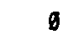 & 19 & 80 & 0 & 6 & $\theta$ & $\theta$ & $\theta$ & \\
\hline PALO VEROE 1 & 49 & 38 & 44 & 37 & 50 & 37 & 40 & 58 & 55 & 34 & 21 & 17 & 19 & 16 & 22 & 16 & 17 & 25 & 24 & \\
\hline PALO VERDE 2 & 58 & 38 & 45 & 37 & 49 & 37 & 40 & 58 & 55 & 35 & 22 & 17 & 20 & 18 & 21 & 16 & 17 & 25 & 24 & 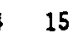 \\
\hline PALO VERDE 3 & 39 & 45 & 46 & 37 & 60 & 35 & 44 & 61 & 34 & 59 & 17 & 20 & 20 & 16 & 26 & 15 & 19 & 27 & 15 & \\
\hline PEACHBOTTON 2 & 8 & 178 & 145 & 0 & 202 & 764 & 0 & $\theta$ & D & ฮ & 6 & 33 & 27 & $\theta$ & 37 & 141 & g & $\theta$ & $\theta$ & \\
\hline PEACHBOTTON 3 & 164 & $\theta$ & 144 & 139 & 0 & 136 & 250 & $\theta$ & 764 & $\theta$ & 30 & 0 & 26 & 25 & $\theta$ & 25 & 46 & g & 139 & \\
\hline PERRY 1 & 191 & $\theta$ & 172 & 132 & 6 & 147 & 153 & 0 & 204 & 127 & 35 & 0 & 31 & 24 & 6 & 27 & 28 & 0 & 37 & \\
\hline PERRY 2 & 224 & $\theta$ & 267 & 154 & 0 & 154 & 250 & $\theta$ & 267 & 231 & 37 & $\boldsymbol{\theta}$ & 38 & 28 & 6 & 28 & 46 & $\theta$ & 49 & 42 \\
\hline PILGRIM 1 & 127 & g & 111 & 580 & $\theta$ & $\theta$ & $\boldsymbol{g}$ & $\theta$ & $g$ & $g$ & 22 & 0 & 20 & 163 & 6 & $\theta$ & $\theta$ & 0 & $\theta$ & \\
\hline POINT BEACH 1 & 21 & 24 & 121 & 0 & 0 & $\theta$ & $\theta$ & $\theta$ & $\theta$ & $\theta$ & 7 & 9 & 43 & $\theta$ & 6 & 6 & $\theta$ & $\theta$ & $\theta$ & \\
\hline POINT BEACH 2 & 21 & 23 & 21 & 121 & $\boldsymbol{\theta}$ & 6 & 6 & 0 & $g$ & $g$ & 7 & 8 & 7 & 43 & $b$ & $\theta$ & $\theta$ & $\theta$ & $\theta$ & \\
\hline PRAIRIE ISL 1 & 26 & $\theta$ & 24 & 24 & 121 & 6 & 0 & 0 & $\theta$ & 0 & 10 & 0 & 9 & 9 & 44 & 0 & $\theta$ & $\theta$ & 0 & \\
\hline PRAIRIE ISL 2 & 27 & 29 & 25 & $\theta$ & 32 & 121 & 0 & $\emptyset$ & $\theta$ & $\theta$ & 9 & 10 & 8 & $\theta$ & 11 & 40 & $g$ & g & 0 & \\
\hline QUAD CITIES 1 & 114 & 129 & 0 & 100 & 724 & 6 & 0 & $g$ & $g$ & $\theta$ & 29 & 23 & 0 & 18 & 128 & $\theta$ & 8 & g & 0 & \\
\hline QUAD CITIES 2 & $\theta$ & 120 & 161 & g & 724 & 6 & 0 & $g$ & $g$ & $\theta$ & $\theta$ & 21 & 18 & $\theta$ & 128 & 0 & $\theta$ & $\theta$ & $\emptyset$ & \\
\hline RANCHO SECO 1 & 0 & 43 & 39 & 31 & $\theta$ & 37 & 60 & $g$ & 46 & 177 & 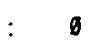 & 29 & 18 & 14 & $g$ & 17 & 28 & $\theta$ & 21 & 82 \\
\hline ROBINSON 2 & 33 & 38 & 157 & 0 & $g$ & $\theta$ & 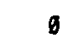 & $\emptyset$ & 9 & $\emptyset$ & 14 & 16 & 68 & 0 & $\theta$ & $b$ & $\theta$ & $\theta$ & $\theta$ & \\
\hline RVR BEND 1 & 117 & 85 & 104 & 81 & 120 & 88 & 95 & 136 & 122 & 77 & 21 & 15 & 19 & 15 & 22 & 16 & 17 & 25 & 22 & 14 \\
\hline SALEM 1 & 49 & $g$ & 46 & 48 & 0 & 45 & 76 & $\theta$ & 55 & 54 & 22 & $g$ & 21 & 21 & $\theta$ & 21 & 35 & 6 & 25 & 25 \\
\hline SALEN 2 & 0 & 56 & 46 & 9 & 63 & 48 & $g$ & 61 & 55 & $\emptyset$ & 0 & 26 & 21 & 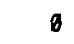 & 29 & 21 & $\theta$ & 28 & 25 & \\
\hline SAN ONOFRE 1 & 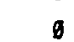 & $\theta$ & g & $g$ & $\theta$ & 0 & $g$ & $\theta$ & $\theta$ & $g$ & $g$ & $g$ & $g$ & 0 & $g$ & $\theta$ & 0 & 6 & 6 & \\
\hline
\end{tabular}


TABLE A.3. Middle Case (with Extended Burnup) 1984 Inventory and Projected Annual Reactor Discharges (contd)

REACTOR

SAN ONOFRE 2

SAN ONOFRE 3

SEABROOK 1

SEABROOK-2

SEQUOYAH 1

SEQUOYAH 2

SHOREHAM

SOUTH TEXAS 1

SOUTH TEXAS 2

ST LUCIE 1

ST LUCIE 2

SUMAER 1

SURRY 1

SURRY 2

SUSQUEHANNA 1

SUSRUETANNA 2

3 MILE ISL 1

TROJAN

TURKEY PT 3

TURKEY PT 4

VDGTLE 1

VOGTLE 2

VT YANKEE 1

WASH NUCLEAR2

WNP-1

WNP-3

WATERFORD 3

WATTS BAR 1

WATTS BAR 2

WOLF CRK 1

YANKEE-ROWE 1

ZION 1

ZION 2

GENERIC PWR

GENERIC EWR

TOTAL GENERIC
Assemblies

NTIHN

\begin{tabular}{|c|c|c|c|c|c|c|c|c|c|c|c|c|c|c|c|c|c|c|c|}
\hline 65 & & & & & & & 2012 & 2 & 2014 & 5 & & 7 & & $\underline{2009}$ & $\underline{2010}$ & 11 & 2012 & 2813 & $\underline{2 \theta}$ \\
\hline 8 & 66 & 8 & 57 & 8 & 58 & $\theta$ & 73 & 8 & 66 & $g$ & 28 & $\theta$ & 24 & $g$ & 25 & $\theta$ & 31 & $a$ & 28 \\
\hline 68 & 8 & 62 & $\theta$ & 75 & & 93 & $\theta$ & 67 & & 26 & & 26 & & 32 & $\theta$ & 46 & & 29 & \\
\hline 58 & 36 & 44 & 32 & 49 & 37 & 46 & 58 & 53 & 33 & & 15 & & 14 & 1 & 16 & 7 & 24 & 2 & 14 \\
\hline 47 & 44 & 45 & 36 & 51 & 35 & 54 & 76 & 7 & 50 & 8 & & 19 & 15 & 2 & 15 & 3 & 18 & 24 & 2. \\
\hline$\theta$ & 58 & & & 67 & & 82 & 63 & 8 & 8 & & & & 22 & 1 & $\theta$ & 88 & 29 & & 27 \\
\hline 52 & 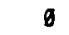 & 47 & 48 & 政 & 46 & 80 & 6 & 60 & & 24 & & 22 & 22 & 8 & 21 & 37 & & 28 & \\
\hline 33 & $\theta$ & 136 & & $\theta$ & 12 & 6 & $\theta$ & 5 & 12 & & & & 9 & $\theta$ & 19 & 18 & & $\theta 0$ & 26 \\
\hline 38 & 3 & 3 & 2 & 47 & 25 & 3 & 47 & 28 & 14 & 16 & 18 & 19 & 14 & 15 & 13 & 18 & 25 & 14 & 24 \\
\hline 36 & 33 & 3 & 27 & 39 & 28 & 41 & 51 & 43 & 37 & 19 & 1 & 19 & 15 & 1 & 15 & 22 & 27 & 23 & 28 \\
\hline 8 & 6 & 5 & 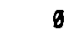 & 73 & 51 & & 68 & 57 & $g$ & 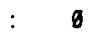 & 21 & & $\theta$ & 23 & 19 & & 22 & 1 & \\
\hline 54 & 8 & 5 & 52 & 6 & 58 & 79 & $\theta$ & 59 & 58 & 20 & & $2 k$ & 28 & v & 19 & 30 & & 22 & 22 \\
\hline 43 & 8 & & 2 & 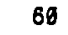 & 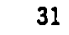 & & 57 & 16 & & & & & 13 & 8 & 4 & & 26 & 1 & 24 \\
\hline 38 & 8 & & 3 & 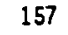 & . & 8 & $\theta$ & $g$ & & 17 & 6 & & 16 & 2 & 8 & 0 & & & \\
\hline 6 & 4 & & & 7 & 8 & 6 & $\theta$ & 0 & $y$ & 8 & & 1 & 8 & 72 & 8 & & & 8 & \\
\hline 157 & & 13 & 35 & 6 & 3 & 39 & 8 & 73 & 5 & 27 & 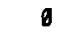 & 24 & 24 & $\varnothing$ & 23 & 42 & & 36 & 31 \\
\hline 8 & & & b & 282 & 13 & $\theta$ & 5 & 2 & & & 3 & 25 & & 6 & 3 & & 34 & 30 & \\
\hline 48 & & & & 6 & 17 & 6 & & $\theta$ & 8 & 22 & & & 18 & 28 & 82 & & & 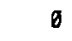 & \\
\hline 26 & 2 & 2 & 2 & 3 & 2 & 46 & 32 & 29 & 29 & 12 & 1 & 11 & 11 & 15 & 11 & 18 & 15 & 13 & 13 \\
\hline 8 & & 3 & & 6 & & & & & $\theta$ & 6 & & & 72 & 8 & & & & & \\
\hline 34 & $\theta$ & 3 & 32 & 157 & 6 & $\theta$ & $\theta$ & b & 8 & 16 & 6 & 15 & 15 & 72 & 8 & g & & 8 & \\
\hline 3 & & & & 6 & & & & & & & & & & & 15 & g & & 16 & 28 \\
\hline 48 & 4 & 4 & & 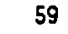 & 32 & 40 & 65 & 34 & 58 & 18 & & & 1 & 27 & 15 & 21 & 30 & 16 & 21 \\
\hline 6 & & & & 8 & & & a & 0 & & 1 & & & 67 & 0 & $\theta$ & & & & \\
\hline 165 & & 9 & 9 & 134 & 88 & 163 & 128 & 116 & 115 & 18 & & & 1 & 24 & 15 & 29 & 13 & 28 & 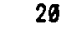 \\
\hline 47 & 4 & 4 & 35 & 51 & 35 & 54 & 76 & 57 & 50 & 21 & & 21 & 16 & 23 & 16 & 25 & 32 & 26 & 23 \\
\hline 5 & 4 & 4 & 3 & 5 & & & 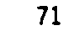 & 58 & & & & & 87 & 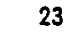 & 14 & 29 & 31 & 25 & 23 \\
\hline 43 & 49 & 46 & $a$ & 88 & 32 & 49 & 64 & $\theta$ & 82 & 18 & & 29 & 8 & 38 & 14 & 21 & 27 & 8 & 35 \\
\hline 63 & 53 & & 7 & $\theta$ & 46 & 70 & & 69 & 73 & & & 6 & & $\theta$ & 21 & 22 & 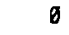 & 32 & 34 \\
\hline 49 & 8 & 5 & 47 & $\theta$ & 45 & 8 & 71 & 44 & 6 & 22 & 8 & 25 & 22 & 0 & 21 & 0 & 33 & 28 & \\
\hline 38 & 8 & 1 & 26 & 37 & 28 & 36 & 43 & 46 & 25 & 18 & 8 & 16 & 12 & 17 & 13 & 14 & 28 & 18 & 12 \\
\hline 8 & 8 & $g$ & 8 & $\theta$ & 8 & $\theta$ & $\theta$ & 6 & $g$ & 6 & 8 & $\theta$ & & $\theta$ & 8 & & & 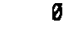 & \\
\hline 33 & 36 & & 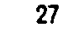 & 193 & 8 & & & 6 & 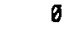 & & & & & 8 & $\theta$ & $\theta$ & 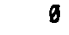 & $\theta$ & \\
\hline 3 & 3 & 2 & 21 & 40 & & $\varnothing$ & 6 & 6 & $\theta$ & 5 & 1 & 10 & 2 & 8 & 88 & g & $g$ & $\theta$ & \\
\hline 447 & 583 & 882 & 693 & 1388 & 1272 & 2393 & 3465 & 3198 & 31 & 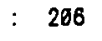 & & 390 & 308 & 18 & 561 & 1825 & 1533 & 1465 & 200 \\
\hline 865 & 1983 & 1273 & 1118 & 2223 & 1634 & 4342 & 5314 & 4754 & 16 & & 1 & 233 & 294 & 486 & 298 & 793 & 970 & 868 & 952 \\
\hline & & & & 3611 & & 6645 & 8779 & 7950 & & & 464 & 629 & 513 & 1024 & 859 & 1818 & 2503 & 2272 & 2233 \\
\hline
\end{tabular}


TABLE A.3. Middle Case (with Extended Burnup) 1984 Inventory and Projected Annual Reactor Discharges (contd)

\begin{tabular}{|c|c|c|c|c|c|c|c|c|c|c|c|c|c|}
\hline \multirow[b]{2}{*}{ REACTOR } & \multicolumn{6}{|c|}{ Assemblies } & \multicolumn{7}{|c|}{ NTIHM } \\
\hline & 2615 & 2616 & 2617 & 2018 & 2819 & 2020 & $:$ & 2015 & 2016 & 2017 & 2018 & 2019 & 2020 \\
\hline ARK NUCLEAR 1 & $\theta$ & $\theta$ & $\theta$ & $\theta$ & 0 & 6 & : & 0 & 0 & 8 & $\theta$ & $\theta$ & 8 \\
\hline ARK NUCLEAR 2 & $\theta$ & $\theta$ & $\theta$ & $\theta$ & $\theta$ & $\theta$ & : & 0 & 0 & $\theta$ & $\theta$ & $\theta$ & 8 \\
\hline B VALLEY 1 & 157 & $\theta$ & $\sigma$ & $\theta$ & 0 & 0 & : & 72 & 0 & 0 & $\theta$ & 0 & $\theta$ \\
\hline B VALLEY 2 & 56 & 46 & $\theta$ & 36 & 52 & 6 & : & 26 & 21 & $\theta$ & 17 & 24 & 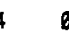 \\
\hline BELLEONTE 1 & 73 & 59 & $\theta$ & 55 & 66 & 6 & : & 33 & 27 & 0 & 25 & 30 & $\theta$ \\
\hline BELEEONTE 2 & 67 & $\theta$ & 68 & 65 & 6 & 58 & : & 31 & $\theta$ & 27 & 30 & $\theta$ & 26 \\
\hline BIG ROCK 1 & $\theta$ & $\boldsymbol{0}$ & $\boldsymbol{\theta}$ & $\theta$ & $\theta$ & 6 & : & 6 & $\theta$ & $\theta$ & $\theta$ & $\theta$ & $\theta$ \\
\hline BRAIDYOOD 1 & 43 & 45 & 36 & 32 & 30 & 53 & : & 18 & 19 & 15 & 14 & 13 & 22 \\
\hline BRAIDYOOD 2 & 42 & 44 & 36 & 32 & 30 & 51 & : & 18 & 19 & 15 & 14 & 13 & 22 \\
\hline BROWNS FERRY1 & $\theta$ & $\theta$ & $\theta$ & $\theta$ & $\theta$ & $\theta$ & : & $\theta$ & $\theta$ & 0 & $\boldsymbol{\theta}$ & 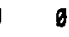 & 8 \\
\hline BROWNS FERRY2 & 764 & $\theta$ & $\theta$ & 6 & 0 & 6 & : & 139 & $\theta$ & 6 & 0 & $\theta$ & 6 \\
\hline BROWNS FERRY3 & 145 & 6 & 234 & 764 & 6 & 6 & : & 26 & 6 & 42 & 89 & 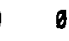 & $b$ \\
\hline BRUNSWICK 1 & 560 & g & 6 & $\theta$ & 0 & $\theta$ & : & 165 & 0 & 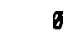 & $\theta$ & $\theta$ & 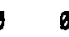 \\
\hline BRUNSWICK 2 & 560 & 0 & 6 & $\theta$ & $\theta$ & $\theta$ & : & 165 & $\theta$ & 0 & $\theta$ & $\theta$ & $\theta$ \\
\hline BYRON 1 & 42 & 45 & 36 & 27 & 31 & 51 & : & 18 & 19 & 15 & 11 & 13 & 22 \\
\hline BYRON 2 & 43 & 44 & 35 & 32 & 31 & 51 & : & 18 & 19 & 15 & 14 & 13 & 22 \\
\hline CALLAIIAY 1 & $\theta$ & 74 & 66 & $\theta$ & 49 & 57 & : & $\theta$ & 31 & 28 & $\theta$ & 21 & 24 \\
\hline CALVERT CLF 1 & $\theta$ & 0 & 6 & 6 & 6 & $\theta$ & : & $\theta$ & $\theta$ & 6 & $\theta$ & 6 & 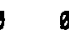 \\
\hline CALVERT CLF 2 & 0 & $\theta$ & 6 & $\theta$ & 6 & $\theta$ & : & 6 & $\theta$ & 6 & $\theta$ & $\theta$ & 6 \\
\hline CARROL LOUNTY-1 & 35 & 39 & 41 & 41 & 33 & 42 & : & 15 & 16 & 17 & 17 & 14 & 18 \\
\hline CARROLL COUNTY-2 & 35 & 39 & 41 & 41 & 33 & 42 & : & 15 & 16 & 17 & 17 & 14 & 18 \\
\hline CATAMEA 1 & 45 & 52 & 39 & 36 & 43 & 38 & : & 19 & 22 & 17 & 15 & 18 & 16 \\
\hline CATAMBA 2 & 44 & 36 & $\theta$ & 34 & 33 & 54 & : & 19 & 15 & 0 & 14 & 14 & 23 \\
\hline CLINTON 1 & $\theta$ & 159 & 112 & $\theta$ & 116 & 136 & : & 6 & 29 & 20 & $\theta$ & 21 & 24 \\
\hline COMANCHE PK 1 & 49 & 51 & 41 & 36 & 36 & 59 & : & 20 & 21 & 17 & 15 & 15 & 24 \\
\hline COMANCHE PK 2 & 50 & 51 & 43 & 35 & 37 & 59 & : & 20 & 21 & 17 & 14 & 15 & 24 \\
\hline $\operatorname{cook} 1$ & $\theta$ & $\theta$ & $\theta$ & $\theta$ & 0 & 0 & : & 6 & 6 & $\theta$ & $\theta$ & $\theta$ & $\theta$ \\
\hline $\operatorname{cook} 2$ & $\theta$ & $\theta$ & $\theta$ & $\theta$ & 0 & $\theta$ & : & $\theta$ & g & $\theta$ & $\theta$ & $\theta$ & 0 \\
\hline COOPER STN & 6 & 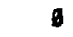 & $\theta$ & $\theta$ & $\theta$ & 6 & : & $\theta$ & $\theta$ & $\boldsymbol{\theta}$ & $\theta$ & 0 & $\theta$ \\
\hline CRYSTAL RVR 3 & $\theta$ & 50 & 45 & 177 & 6 & $\theta$ & $:$ & $\theta$ & 23 & 21. & 82 & 0 & $\theta$ \\
\hline DAVIS-BESSE 1 & $\theta$ & 60 & 58 & 177 & 6 & 6 & : & $\theta$ & 28 & 26 & 83 & 0 & $\theta$ \\
\hline DIABLO CNYN 1 & 37 & 53 & 51 & $\theta$ & 29 & 48 & : & 17 & 24 & 23 & 6 & 13 & 22 \\
\hline DIABLO CNYN 2 & 43 & $\theta$ & 41 & 29 & 50 & 36 & : & 20 & $\boldsymbol{\theta}$ & 19 & 13 & 23 & 17 \\
\hline DRESDEN 1 & 6 & $\theta$ & $\theta$ & $\theta$ & g & $\theta$ & : & $\theta$ & $\theta$ & 0 & 6 & 6 & 0 \\
\hline DRESDEN 2 & 6 & $\theta$ & $\theta$ & $\theta$ & 0 & $\theta$ & : & $\theta$ & $\theta$ & $\theta$ & $\theta$ & $\theta$ & $\theta$ \\
\hline DRESDEN 3 & 6 & $\theta$ & $\theta$ & $\theta$ & $\theta$ & $\theta$ & : & $\theta$ & $\theta$ & $\theta$ & $\theta$ & 6 & 6 \\
\hline DUANE ARNOLD & 368 & $\theta$ & $\theta$ & $\theta$ & 0 & $\theta$ & : & 67 & $\theta$ & $\theta$ & 6 & $\theta$ & 6 \\
\hline ENRICO FERMI2 & $\theta$ & 233 & 150 & $\theta$ & 155 & 0 & : & $\theta$ & 42 & 27 & 0 & 28 & 6 \\
\hline FARLEY 1 & 6 & $\theta$ & $\theta$ & $\theta$ & $\theta$ & $\theta$ & : & $\theta$ & $\theta$ & $\theta$ & 6 & 6 & 6 \\
\hline FARLEY 2 & 6 & 54 & 157 & 0 & $\theta$ & 0 & : & 0 & 25 & 72 & 0 & 0 & 0 \\
\hline FITZPATRICK & 566 & $\theta$ & $\theta$ & $\theta$ & $\theta$ & 6 & : & 101 & 0 & 0 & 6 & 6 & 6 \\
\hline FORT CALHOUN & 6 & $\theta$ & $\theta$ & $\theta$ & $\theta$ & 0 & ; & $\theta$ & $\theta$ & $\theta$ & 6 & 6 & $\theta$ \\
\hline GINNA & 6 & $\theta$ & $\theta$ & $\theta$ & 0 & 0 & : & $\theta$ & 0 & $\theta$ & 0 & 0 & 6 \\
\hline GRAND GULF 1 & 131 & 238 & $\theta$ & 163 & 151 & 173 & : & 23 & 42 & 0 & 29 & 27 & 30 \\
\hline GRANO GULF 2 & 139 & 163 & 165 & 165 & 136 & 171 & : & 26 & 30 & 30 & 30 & 25 & 31 \\
\hline HAODAM NECK & $\theta$ & $\boldsymbol{\theta}$ & $\theta$ & $\theta$ & 6 & 6 & $:$ & 6 & 0 & 0 & 6 & 6 & 6 \\
\hline HARRIS 1 & $\theta$ & 41 & 0 & 31 & 36 & 0 & : & 0 & 19 & $\theta$ & 14 & 17 & $\theta$ \\
\hline HATCH 1 & 0 & 0 & $\theta$ & 6 & 6 & 6 & : & 0 & $\theta$ & $\theta$ & 0 & $\theta$ & $\theta$ \\
\hline
\end{tabular}


TABLE A.3. Middle Case (with Extended Burnup) 1984 Inventory and Projected Annual Reactor Discharges (contd)

\begin{tabular}{|c|c|c|c|c|c|c|c|c|c|c|c|c|c|}
\hline \multirow[b]{2}{*}{ REACTOR } & \multicolumn{6}{|c|}{ Assemblies } & \multicolumn{7}{|c|}{ MTIHM } \\
\hline & 2015 & 2016 & 2017 & 2018 & 2019 & 2020 & : & 2015 & 2016 & 2017 & 2018 & 2019 & 2020 \\
\hline HATCH 2 & $\theta$ & 6 & 6 & 0 & $\theta$ & 0 & : & $\theta$ & $\theta$ & 0 & 0 & $\theta$ & 0 \\
\hline HOPE CRK 1 & 203 & 168 & 0 & 154 & 189 & 0 & : & 37 & 31 & 6 & 28 & 34 & $a$ \\
\hline HUMBOLDT BAY & 6 & 6 & 0 & $\theta$ & $\theta$ & 0 & : & $\theta$ & $\theta$ & 6 & $\theta$ & 0 & 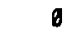 \\
\hline INDIAN PT 1 & 6 & 0 & 0 & $\theta$ & 0 & 0 & : & 0 & 0 & 0 & 6 & 0 & a \\
\hline INDIAN PT 2 & $\theta$ & 6 & 6 & 6 & 6 & 0 & : & 0 & 0 & 0 & 0 & 0 & 6 \\
\hline INDIAN PT 3 & $\theta$ & 62 & 193 & 6 & 6 & 0 & : & 6 & 28 & 88 & 6 & 0 & 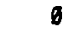 \\
\hline KEIAUNEE & 6 & $\theta$ & $\theta$ & 0 & $g$ & $\theta$ & : & 0 & $\theta$ & $\theta$ & 6 & $\theta$ & 0 \\
\hline LACROSSE & 0 & 6 & 0 & 9 & 6 & 0 & : & 6 & 6 & 0 & 0 & 0 & 0 \\
\hline LASALLE CTY 1 & 117 & 6 & 184 & 144 & 6 & 196 & : & 21 & $\theta$ & 34 & 28 & ด & 35 \\
\hline LASALLE CTY 2 & 117 & 195 & $\theta$ & 139 & 124 & $\theta$ & : & 21 & 36 & 0 & 25 & 23 & 0 \\
\hline LIMERICK 2 & 213 & 0 & 207 & 219 & 9 & 199 & : & 39 & 6 & 38 & 40 & $\theta$ & 36 \\
\hline LIMERICK 1 & 168 & 135 & 0 & 125 & 154 & $\theta$ & : & 31 & 25 & $\theta$ & 23 & 28 & 0 \\
\hline MAINE YANKEE & $\theta$ & 6 & $\theta$ & 0 & 0 & 0 & : & 6 & $\theta$ & $\theta$ & 0 & $\theta$ & $\theta$ \\
\hline MCGUIRE 1 & 32 & 54 & 52 & 32 & 44 & 6 & : & 14 & 23 & 22 & 14 & 19 & 6 \\
\hline MCOUIRE 2 & 33 & 9 & 50 & 37 & 35 & 40 & : & 14 & $\theta$ & 21 & 16 & 15 & 17 \\
\hline MILSTONE 1 & $\theta$ & 6 & 6 & 6 & 6 & 6 & : & 9 & 6 & 6 & 0 & $\theta$ & $\theta$ \\
\hline MILLSTONE 2 & 217 & 6 & 6 & 6 & 6 & 6 & : & 88 & 6 & $\theta$ & 0 & 6 & 6 \\
\hline MILLSTONE 3 & 43 & 40 & 38 & 6 & 34 & 57 & : & $2 a$ & 18 & 18 & g & 16 & 26 \\
\hline NONTICELO & 6 & 0 & 6 & 6 & 0 & 6 & : & 6 & $\theta$ & $\theta$ & 0 & 0 & $\theta$ \\
\hline NINE MILE PT1 & 0 & 6 & 6 & 6 & 6 & $\theta$ & : & 6 & 0 & $\theta$ & 0 & $\theta$ & 6 \\
\hline NINE MILE PT2 & 6 & 214 & 6 & 156 & 188 & 6 & : & 6 & 38 & 0 & 27 & 34 & $\theta$ \\
\hline NORTH ANNA 1 & 0 & 63 & 157 & 0 & 6 & 0 & : & 0 & 29 & 72 & 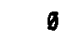 & 0 & 6 \\
\hline NORTH ANNA 2 & 55 & 0 & 157 & 6 & 6 & 6 & : & 25 & $\emptyset$ & 72 & 6 & 6 & 6 \\
\hline OCONEE 1 & $\theta$ & $\theta$ & $\theta$ & $\emptyset$ & 6 & 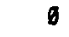 & : & b & D & D & $\theta$ & $\theta$ & 0 \\
\hline DCONEE 2 & 0 & $\theta$ & $\theta$ & 6 & 6 & 6 & : & 0 & 0 & 0 & 6 & 6 & 6 \\
\hline DCONEE 3 & 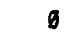 & $\theta$ & 6 & 6 & 6 & 0 & : & 0 & $\emptyset$ & 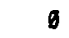 & $g$ & $\theta$ & 6 \\
\hline OYSTER CRK 1 & 0 & $\theta$ & $\theta$ & 6 & 6 & 6 & : & 0 & 0 & 0 & 0 & $b$ & 6 \\
\hline PALISADES & $\theta$ & 0 & 6 & 6 & 0 & $\theta$ & : & 0 & 6 & $\theta$ & 6 & 6 & 0 \\
\hline PALO YERDE 1 & 49 & 51 & 41 & 36 & 36 & 58 & : & 21 & 22 & 18 & 16 & 16 & 25 \\
\hline PALO YERDE 2 & 47 & 50 & 40 & 38 & 36 & 58 & : & 21 & 22 & 17 & 17 & 16 & 25 \\
\hline PALO VERDE 3 & 44 & 40 & 34 & 42 & 46 & 39 & : & 19 & 17 & 15 & 18 & 20 & 17 \\
\hline PEACHBOTTOM 2 & $\theta$ & 6 & $\theta$ & 6 & 6 & 6 & : & 6 & 0 & $\theta$ & $\theta$ & $\theta$ & 6 \\
\hline PEACHBOTTOM 3 & 6 & 0 & 6 & 6 & 6 & 6 & $:$ & 0 & 0 & $\theta$ & g & 0 & 6 \\
\hline PERRY 1 & 6 & 196 & 155 & 6 & 133 & 217 & : & 0 & 36 & 28 & $g$ & 24 & 39 \\
\hline PERRY 2 & 6 & 223 & 226 & $\theta$ & 186 & 235 & : & 6 & 41 & 41 & 0 & 34 & 43 \\
\hline PILGRIM 1 & 6 & $\theta$ & 6 & $\theta$ & B & $\theta$ & : & $\theta$ & $\theta$ & 0 & g & $\theta$ & $\theta$ \\
\hline POINT BEACH 1 & 0 & 0 & 6 & 6 & $\theta$ & $\theta$ & : & $\theta$ & 0 & $\theta$ & $\theta$ & $\theta$ & 6 \\
\hline POINT BEACH 2 & 6 & 6 & 0 & 6 & 6 & $\theta$ & : & 6 & $\theta$ & $\theta$ & $\theta$ & $\theta$ & $\theta$ \\
\hline PRAIRIE ISL 1 & $\theta$ & 0 & $\theta$ & 6 & 0 & 0 & : & 6 & 6 & $\theta$ & $\theta$ & $\theta$ & $z$ \\
\hline PRAIRIE ISL 2 & $\theta$ & 6 & $\theta$ & $\theta$ & 6 & $\theta$ & : & 6 & $\theta$ & $\theta$ & g & $\theta$ & $\theta$ \\
\hline QUAD CITIES 1 & $\theta$ & 6 & $\theta$ & 0 & 0 & $\theta$ & : & 6 & $\theta$ & $\theta$ & $\theta$ & 0 & $\theta$ \\
\hline QUAD CITIES 2 & $\emptyset$ & 6 & 0 & 0 & 6 & б & : & $\theta$ & 6 & $\theta$ & 0 & $\theta$ & $\theta$ \\
\hline RANCHO SECO 1 & 6 & 6 & 6 & $\theta$ & 0 & 0 & : & 6 & 6 & $\theta$ & 0 & $\theta$ & $\theta$ \\
\hline ROBINSON 2 & $\theta$ & 6 & 6 & $\theta$ & $\theta$ & 6 & : & 6 & 0 & 0 & $\theta$ & $\theta$ & $\theta$ \\
\hline RYR BEND 1 & 112 & 126 & 95 & 88 & 83 & 143 & : & 26 & 22 & 17 & 16 & 15 & 25 \\
\hline SALEI 1 & 6 & 68 & 64 & 193 & $\theta$ & 6 & : & $\theta$ & 31 & 29 & 89 & $\theta$ & 0 \\
\hline SALEN 2 & 43 & 67 & $\theta$ & 50 & 56 & 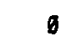 & : & 20 & 31 & $\theta$ & 23 & 26 & $\theta$ \\
\hline SAN ONOFRE 1 & 6 & 0 & $\theta$ & $\theta$ & $\theta$ & g & : & $\theta$ & $\theta$ & $\theta$ & 6 & $\theta$ & 0 \\
\hline
\end{tabular}


TABLE A.3. Middle Case (with Extended Burnup) 1984 Inventory and Projected Annual Reactor Discharges (contd)

\begin{tabular}{|c|c|c|c|c|c|c|c|c|c|c|c|c|c|}
\hline \multirow[b]{2}{*}{ REACTOR } & \multicolumn{6}{|c|}{ Assemblies } & \multicolumn{7}{|c|}{ MTIHM } \\
\hline & $\underline{2015}$ & $\underline{2016}$ & 2017 & 2018 & 2019 & $\underline{2020}$ & & $\underline{2615}$ & 2016 & 2017 & 2918 & 2019 & 2020 \\
\hline SAN ONOFRE 2 & $\theta$ & 61 & 0 & 73 & g & 68 . & : & o & $\because 6$ & 0 & 31 & 0 & 29 \\
\hline SAN ONOFRE 3 & 57 & 59 & 0 & 58 & 8 & 73 & : & 24 & $: 15$ & 0 & 25 & 0 & 31 \\
\hline SEABROOK 1 & 48 & 52 & 41 & 30 & 34 & 56 & : & 26 & $\because ! 2$ & 17 & 13 & 14 & 24 \\
\hline SEABROOK-2 & 41 & 48 & 49 & 49 & 48 & 51 & $:$ & 17 & $\because 6$ & 21 & 21 & 17 & 22 \\
\hline SEQUOYAH 1 & 0 & 69 & 45 & $\theta$ & 46 & 6 & : & 6 & :12 & 21 & 0 & 21 & $\theta$ \\
\hline SEQUOYAH 2 & 42 & 69 & $\theta$ & 51 & 44 & 0 & : & 19 & :i2 & 0 & 23 & 20 & 6 \\
\hline SHOREHAM & 0 & 138 & 138 & g & 115 & 145 & ; & $\theta$ & $: 25$ & 25 & $\theta$ & 21 & 26 \\
\hline SOUTH TEXAS 1 & 34 & 30 & 27 & 32 & 34 & 29 & : & 18 & .6 & 15 & 17 & 18 & 16 \\
\hline SOUTH TEXAS 2 & 31 & 36 & 38 & 37 & 31 & 39 & : & 17 & $: 9$ & 20 & 26 & 17 & 21 \\
\hline ST LUUCIE 1 & 217 & g & 0 & 0 & $\theta$ & 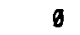 & : & 83 & 6 & g & $\theta$ & 0 & $\theta$ \\
\hline ST LUUCIE 2 & 8 & 71 & 66 & $\theta$ & 48 & 56 & : & $\theta$ & $? 7$ & 25 & 6 & 18 & 21 \\
\hline SUIMMER 1 & 25 & 0 & 61 & 39 & 38 & 43 & : & 11 & 6 & 28 & 18 & 17 & 20 \\
\hline SURRY 1 & $\theta$ & $\theta$ & $\theta$ & $\theta$ & $\theta$ & 8 & $:$ & 0 & $\theta$ & $\theta$ & 6 & 6 & 8 \\
\hline SURRY 2 & 9 & 9 & $\theta$ & 0 & 0 & 9 & : & 9 & $\theta$ & g & 8 & 9 & 9 \\
\hline SUSQUEHANNA 1 & $\theta$ & 212 & 194 & $\theta$ & 135 & 169 & $\cdot$ & $\theta$ & 37 & 34 & 6 & 24 & 30 \\
\hline SUSQUEHANNA 2 & 124 & 269 & $g$ & 150 & 137 & 6 & : & 22 & 37 & 0 & 26 & 24 & 6 \\
\hline 3 MILE ISL 1 & $\theta$ & 0 & 9 & $\theta$ & $\theta$ & 0 & . & 0 & 0 & 9 & 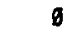 & 0 & 8 \\
\hline TROJAN & 21 & 193 & g & $\theta$ & 0 & 0 & : & 10 & 39 & 0 & 0 & 0 & 0 \\
\hline TURKEY PT 3 & 8 & 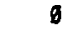 & 8 & 9 & 0 & 0 & : & 6 & 6 & 6 & 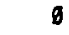 & 0 & 6 \\
\hline TURKEY PT 4 & 9 & 9 & 9 & $\theta$ & $\theta$ & $\theta$ & $r^{\prime}$ & 6 & 9 & 9 & 9 & 6 & $\theta$ \\
\hline VOGTLE 1 & 46 & 40 & 32 & 42 & 45 & 39 & : & 21 & 18 & 15 & 19 & 21 & 18 \\
\hline VOGTLE 2 & 43 & 40 & 32 & 42 & 46 & 46 & . & 20 & 18 & 15 & 19 & 21 & 18 \\
\hline VT YANKEE 1 & $\theta$ & $\theta$ & $\theta$ & $\theta$ & $\theta$ & $\theta$ & : & $\theta$ & $\theta$ & $\theta$ & $\theta$ & 9 & $\theta$ \\
\hline WASH NUCLEAR2 & 85 & 141 & 133 & 108 & 92 & 110 & : & 15 & 25 & 23 & 18 & 16 & 19 \\
\hline NP -1 & 41 & 48 & 49 & 49 & 40 & 51 & : & 19 & 22 & 22 & 22 & 18 & 23 \\
\hline WP-3 & 43 & 50 & 51 & 51 & 42 & 53 & $:$ & 19 & .22 & 22 & 22 & 18 & 23 \\
\hline ATERFORD 3 & 43 & 44 & 48 & 41 & $g$ & 53 & : & 18 & 19 & 17 & 18 & 0 & 23 \\
\hline WATTS BAR 1 & $\theta$ & 63 & $g$ & 47 & 44 & 0 & : & $\theta$ & 29 & 9 & 22 & 20 & $\theta$ \\
\hline WATTS BAR 2 & 81 & 49 & 9 & 52 & 8 & 69 & : & 28 & .22 & 0 & 24 & $\theta$ & 32 \\
\hline NOLF CRK 1 & 36 & 37 & 31 & 27 & 28 & 43 & : & 17 & 17 & 14 & 12 & 12 & 28 \\
\hline YANKEE-ROWE 1 & $\theta$ & 9 & 8 & $\theta$ & 9 & $\theta$ & : & $\theta$ & 0 & 9 & 0 & $\theta$ & g \\
\hline ZION 1 & 6 & 9 & 8 & 9 & 8 & $\theta$ & : & $\theta$ & 0 & $\theta$ & 0 & $\theta$ & g \\
\hline ZION 2 & 6 & 6 & $\theta$ & 8 & 8 & $\theta$ & : & $\theta$ & 0 & g & 6 & 9 & g \\
\hline GENERIC PWR & 2763 & 3475 & 3548 & 3930 & 3571 & 4272 & : & 1260 & $15: 36$ & 1531 & 1695 & 1536 & 1835 \\
\hline GENERIC BWR & 3797 & 5268 & 6648 & 5726 & 5138 & 7262 & : & 692 & 9131 & 1162 & 1844 & 936 & 1323 \\
\hline TOTAL GENERIC & 6560 & 8743 & 9596 & 9656 & 8769 & 11534 & & 1893 & 24.57 & 2633 & 2739 & 2472 & 3158 \\
\hline
\end{tabular}


TABLE A.3. Middle Case (with Extended Burnup) 1984 Inventory and Projected Annual Reactor Discharges (contd)

\begin{tabular}{|c|c|c|c|c|c|c|c|c|c|c|c|c|}
\hline & & & & & & TOTALS & REACTO & DE AND & ALS & & & \\
\hline & & & & & & $-\ldots$ & $-\cdots-$ & $-\cdots$ & & & & \\
\hline & & 1984 & 1985 & 1986 & 1987 & 1988 & 1989 & 1996 & 1991 & 1992 & 1993 & 1994 \\
\hline & & --- & ---- & --- & $\cdots$ & --- & $\cdots$ & $\cdots$ & $\cdots$ & --- & $\cdots$ & ---- \\
\hline PWR & ASSEMBLY & 16087 & 1673 & 2628 & 2871 & 3843 & 2435 & 2785 & 3096 & 2437 & 3714 & 2144 \\
\hline PWR & NT. & 6787 & 713 & 890 & 898 & 1299 & 1863 & 1169 & 1321 & 1062 & 1624 & 917 \\
\hline BWR & ASSEYBLY & 25461 & 1662 & 3604 & 2553 & 4101 & 3855 & 2583 & 4714 & 2879 & 4758 & 3652 \\
\hline BWR & NT. & 4635 & 300 & 654 & 463 & 744 & 700 & 466 & 853 & 519 & 855 & 550 \\
\hline TOTAL & ASSEMBLY & 41548 & 3335 & 5632 & 4624 & 7144 & 6290 & 5288 & 7810 & 5316 & 8472 & 5194 \\
\hline TOTAL & NT. & 11423 & 1813 & 1544 & 1360 & 2943 & 1764 & 1635 & 2174 & 1581 & 2478 & 1467 \\
\hline
\end{tabular}

\begin{tabular}{|c|c|c|c|c|c|c|c|c|c|c|c|}
\hline & & & & & SUBT & S BY R & OR TYP & TOTA & & & \\
\hline & & 1995 & 1996 & 1997 & 1998 & 1999 & 2000 & 2001 & 2002 & 2003 & 2004 \\
\hline & & ---- & --- & $-\cdots$ & $-\cdots$ & $\cdots$ & ---- & --- & --- & --- & $-\cdots$ \\
\hline PWR & ASSEMBLY & 4854 & 2262 & 3884 & 2928 & 2633 & 3721 & 2359 & 4856 & 2471 & 3938 \\
\hline PUR & NT. & 1771 & 983 & 1868 & 1263 & 1148 & 1605 & 1634 & 1769 & 1870 & 1739 \\
\hline BNR & ASSEMBLY & 5861 & 3238 & 4292 & 4116 & 3628 & 4894 & 3281 & 5236 & 3314 & 5299 \\
\hline BHR & MT. & 916 & 583 & 776 & 742 & 544 & 882 & 592 & 939 & 599 & 956 \\
\hline TOTAL & ASSEMBLY & 9115 & 5498 & 8156 & 7044 & 5653 & 8615 & 5648 & 9292 & 5785 & 9237 \\
\hline TOTAL & MT. & 2687 & 1568 & 2442 & 2006 & 1692 & 2487 & 1626 & 2708 & 1669 & 2695 \\
\hline
\end{tabular}

\begin{tabular}{|c|c|c|c|c|c|c|c|c|c|c|c|}
\hline & & & & & SUBT & LS BY RI & TOR TYP & ND TOTAL & & & \\
\hline & & 2005 & 2006 & 2007 & 2008 & 2009 & 2010 & 2011 & 2012 & 2013 & 2014 \\
\hline & & -.-- & $-\cdot--$ &.-- & --.- & ---- & --- & --- & ---- & --- & --- \\
\hline PWR & ASSEMBLY & 3355 & 3021 & 4366 & 3585 & 5008 & 4635 & 4837 & 6180 & 6106 & 5919 \\
\hline PWR & MT. & 1473 & 1320 & 1902 & 1518 & 2200 & 2045 & 2143 & 2726 & 2685 & 2522 \\
\hline BWR & ASSEMBLY & 5688 & 4159 & 7771 & 4893 & 7281 & 5494 & 7516 & 8583 & 8500 & 8883 \\
\hline BWR & NT. & 918 & 754 & 1481 & 888 & 1391 & 1081 & 1369 & 1562 & 1548 & 1619 \\
\hline TOTA & ASSEMBLY & 8443 & 7180 & 12137 & 8398 & 12209 & 16129 & 12353 & 14763 & 14606 & 14802 \\
\hline TOTA & MT. & 2391 & 2874 & 3303 & 2407 & 3501 & 3046 & 3512 & 4289 & 4233 & 4141 \\
\hline
\end{tabular}


TABLE A.3. Middle Case (with Extended Burnup) 1984 Inventory and Projected Annual Reactor Discharges (contd)

\begin{tabular}{|c|c|c|c|c|c|c|c|}
\hline & & & SUBTOT/ & DY REAC & TYPE A & TOTALS & \\
\hline & & & --- & $\cdots$ & $\cdots$ & ........... & \\
\hline & & 2015 & 2818 & 2017 & 2018 & 2019 & 2020 \\
\hline & & --- & $\cdots$ & $\cdots$ & --- & $-\infty$ & --- \\
\hline PWR & ASSEMBLY & 5816 & 5912 & 5768 & 5994 & 5035 & 5986 \\
\hline PWR & MT. & 2181 & 2598 & 2518 & 2623 & 2185 & 2584 \\
\hline BirR & ASSENBLY & 8163 & 8012 & 8841 & 8687 & 7232 & 9144 \\
\hline BiWR & MT. & 1490 & 1456 & 1463 & 1421 & 1314 & 1662 \\
\hline TOTA & ASSENBLY & 13179 & 13924 & 13881 & 14881 & 12267 & 15136 \\
\hline TOTA & MT. & 3671 & 4846 & 3982 & 4643 & 3500 & 4246 \\
\hline
\end{tabular}


REACTOR

ARK NUCLEAR 1

ARK NUCLEAR 2

B VALLEY 1

8 VALLEY 2

BELLEONTE 1

BELLEONTE 2

BIG ROCK 1

BRAIDYOOD 1

BRAIDYOOD 2

BROWNS FERRY1

BROWNS FERRY2

BROWNS FERRY3

BRUNSWICK 1

BRUNSWICK 2

BYRON 1

BYRON 2

CALLAMAY 1

CALVERT CLF 1

CALVERT CLF 2

CARROLL COUNTY-1

CARROLL COUNTY-2

CATAWBA 1

CATAFBA 2

CLINTON 1

COMANCHE PK 1

COMANCHE PK 2

COOK 1

COOK 2

COOPER STN

CRYSTAL RVR 3

DAVIS-BESSE 1

DIABLD CNYN 1

DIABLO CNYN 2

DRESDEN 1

DRESDEN 2

DRESDEN 3

DUANE ARNOLD

ENRICO FERMI2

FARLEY 1

FARLEY 2

FITZPATRICK

FORT CALHOUN

GINNA

GRAND GULF 1

GRAND GULF 2

HADDAM NECK

HARRIS 1

HATCH 1
Inv. Assemblies _. Inv. MTIHM

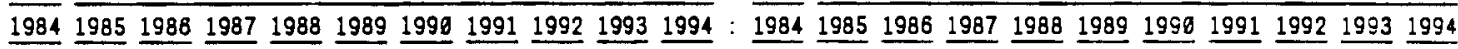

$\begin{array}{lllllllllllllllllllllll}384 & 384 & 435 & 435 & 492 & 492 & 538 & 602 & 602 & 658 & 688 & : & 178 & 178 & 292 & 202 & 228 & 228 & 249 & 279 & 279 & 318 & 310\end{array}$

$\begin{array}{lllllllllllllllllllllll}160 & 212 & 268 & 268 & 329 & 329 & 379 & 447 & 447 & 516 & 516 & : & 66 & 88 & 112 & 112 & 138 & 138 & 159 & 187 & 187 & 216 & 215\end{array}$

$\begin{array}{lllllllllllllllllllllll}218 & 218 & 275 & 322 & 322 & 373 & 426 & 426 & 464 & 535 & 535 & 100 & 100 & 126 & 148 & 148 & 171 & 196 & 196 & 213 & 246 & 246\end{array}$

$\begin{array}{lllllllllllllllllllllll}6 & 0 & 0 & 0 & 0 & 39 & 39 & 112 & 112 & 171 & 171 & : & 0 & 0 & 0 & 0 & 0 & 18 & 18 & 52 & 52 & 79 & 79\end{array}$

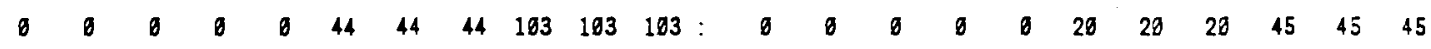

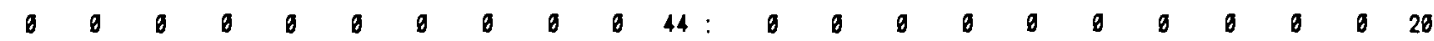

$\begin{array}{lllllllllllllllllllllll}172 & 189 & 265 & 222 & 222 & 239 & 256 & 276 & 291 & 312 & 328 & : & 22 & 25 & 27 & 29 & 29 & 31 & 33 & 36 & 38 & 41 & 43\end{array}$

$\begin{array}{llllllllllllllllllllll}6 & 0 & 6 & 76 & 123 & 157 & 265 & 241 & 295 & 325 & : & 6 & 0 & 0 & 0 & 30 & 52 & 66 & 87 & 102 & 125 & 137\end{array}$

$\begin{array}{llllllllllllllllllllll}0 & 0 & 0 & 0 & 42 & 84 & 135 & 169 & 223 & 252: & 0 & 0 & 0 & 0 & 0 & 18 & 36 & 57 & 71 & 94 & 107\end{array}$

$\begin{array}{lllllllllllllllllllllll}1068 & 1256 & 1256 & 1453 & 1453 & 1635 & 1635 & 1635 & 1783 & 1783 & 1953 & : & 281 & 235 & 235 & 271 & 271 & 394 & 394 & 394 & 331 & 331 & 362\end{array}$

$\begin{array}{llllllllllllllllllllllll}1188 & 1188 & 1363 & 1363 & 1363 & 1531 & 1531 & 1798 & 1798 & 2869 & 2069 & : & 222 & 222 & 254 & 254 & 254 & 285 & 285 & 334 & 334 & 383 & 383\end{array}$

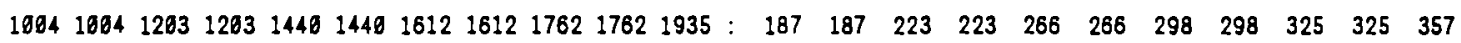
$\begin{array}{lllllllllllllllllllllll}512 & 512 & 573 & 573 & 768 & 829 & 829 & 996 & 1088 & 1088 & 1198: & 96 & 96 & 107 & 107 & 132 & 155 & 155 & 186 & 283 & 283 & 224\end{array}$ $\begin{array}{lllllllllllllllllllllll}752 & 752 & 893 & 998 & 998 & 1119 & 1247 & 1247 & 1341 & 1512 & 1512 & : & 141 & 141 & 167 & 187 & 187 & 209 & 233 & 233 & 251 & 283 & 283\end{array}$

$\begin{array}{llllllllllllllllllllll}0 & 50 & 83 & 131 & 175 & 216 & 265 & 299 & 353 & 383 & 0 & 0 & 0 & 21 & 35 & 55 & 74 & 91 & 112 & 126 & 149 & 162\end{array}$

$\begin{array}{lllllllllllllllllllllll}0 & 0 & 0 & 33 & 81 & 122 & 168 & 210 & 245 & 298 & 327 & 0 & 0 & 0 & 0 & 14 & 34 & 52 & 68 & 89 & 184 & 126 & 138\end{array}$

$\begin{array}{lllllllllllllllllllllll}0 & 0 & 49 & 103 & 103 & 160 & 216 & 218 & 265 & 343 & 343 & : & 0 & 0 & 23 & 47 & 47 & 73 & 96 & 96 & 117 & 150 & 158\end{array}$

$\begin{array}{lllllllllllllllllllllll}468 & 535 & 592 & 592 & 663 & 715 & 715 & 785 & 830 & 830 & 877 & : & 179 & 265 & 227 & 227 & 255 & 275 & 275 & 363 & 320 & 320 & 339\end{array}$

$\begin{array}{lllllllllllllllllllllll}391 & 448 & 448 & 499 & 578 & 578 & 633 & 793 & 763 & 773 & 829 & : & 149 & 172 & 172 & 191 & 222 & 222 & 243 & 271 & 271 & 298 & 317\end{array}$

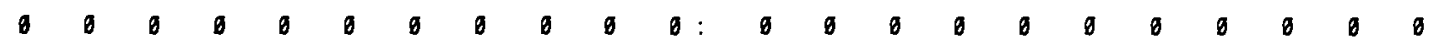

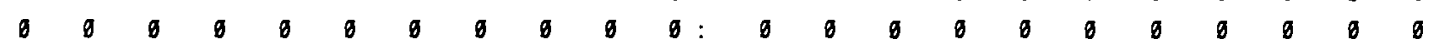

$\begin{array}{lllllllllllllllllllllll}0 & 37 & 37 & 79 & 126 & 171 & 214 & 271 & 387 & 357 & 357 & 0 & 0 & 16 & 16 & 33 & 53 & 72 & 91 & 115 & 136 & 151 & 151\end{array}$

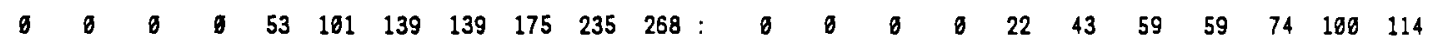

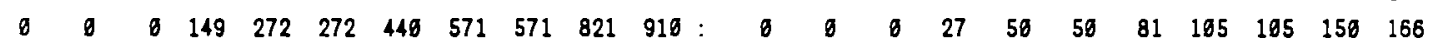

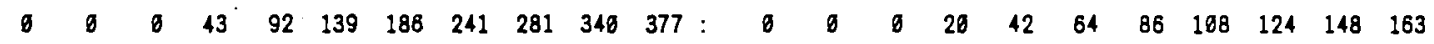

$\begin{array}{llllllllllllllllllllll}0 & 0 & 16 & 96 & 143 & 191 & 247 & 285 & 347 & 385 & : & 0 & 0 & 9 & 17 & 41 & 60 & 81 & 103 & 119 & 144 & 159\end{array}$

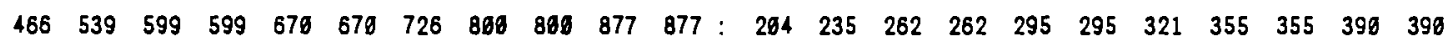

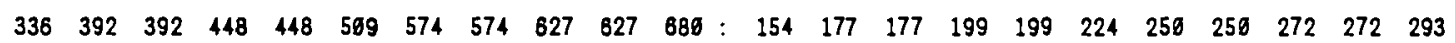

$\begin{array}{lllllllllllllllllllllll}914 & 914 & 1919 & 1982 & 1192 & 1271 & 1355 & 1467 & 1534 & 1647 & 1719 & : & 172 & 172 & 191 & 283 & 223 & 237 & 253 & 273 & 285 & 386 & 319\end{array}$

$\begin{array}{lllllllllllllllllllllll}253 & 276 & 367 & 346 & 346 & 386 & 422 & 422 & 454 & 567 & 567 & : & 118 & 126 & 143 & 161 & 181 & 179 & 196 & 196 & 211 & 235 & 235\end{array}$

$\begin{array}{lllllllllllllllllllllll}197 & 197 & 246 & 293 & 293 & 337 & 385 & 385 & 421 & 489 & 489 & 9 & 93 & 93 & 116 & 138 & 138 & 159 & 181 & 181 & 198 & 230 & 236\end{array}$

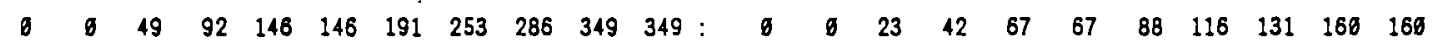

$\begin{array}{lllllllllllllllllllllll}6 & 0 & 53 & 96 & 96 & 146 & 189 & 249 & 287 & 337 & 337 & : & 0 & 6 & 24 & 44 & 44 & 67 & 87 & 114 & 132 & 155 & 155\end{array}$

$\begin{array}{lllllllllllllllllllllll}683 & 683 & 683 & 683 & 683 & 683 & 583 & 683 & 683 & 683 & 683 & : & 69 & 69 & 69 & 69 & 69 & 69 & 69 & 69 & 69 & 69 & 69\end{array}$ $\begin{array}{lllllllllllllllllllllll}1312 & 1312 & 1423 & 1545 & 1545 & 1676 & 1815 & 1815 & 1917 & 2193 & 2163 & : & 247 & 247 & 267 & 289 & 289 & 312 & 337 & 337 & 356 & 389 & 389\end{array}$ $\begin{array}{lllllllllllllllllllllll}1168 & 1260 & 1367 & 1367 & 1510 & 1613 & 1613 & 1759 & 1838 & 1838 & 1935 & : & 222 & 239 & 259 & 259 & 285 & 304 & 384 & 331 & 345 & 345 & 363\end{array}$

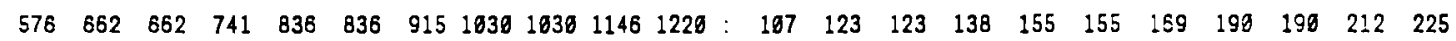

$\begin{array}{lllllllllllllllllllllll}6 & 0 & 206 & 362 & 362 & 581 & 581 & 837 & 837 & 1109 & 1169 & : & 6 & 0 & 38 & 67 & 67 & 187 & 107 & 153 & 153 & 283 & 203\end{array}$

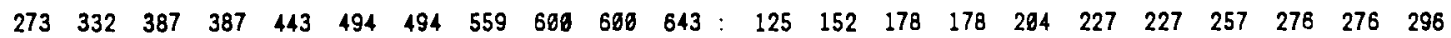
$\begin{array}{lllllllllllllllllllllll}116 & 173 & 229 & 274 & 274 & 320 & 373 & 373 & 411 & 481 & 481 & : & 53 & 79 & 165 & 126 & 126 & 147 & 171 & 171 & 189 & 221 & 221\end{array}$ $\begin{array}{lllllllllllllllllllllll}816 & 970 & 1092 & 1092 & 1254 & 1371 & 1371 & 1530 & 1629 & 1629 & 1733 & : & 153 & 181 & 204 & 284 & 233 & 255 & 255 & 283 & 301 & 301 & 320\end{array}$ $\begin{array}{lllllllllllllllllllllll}385 & 352 & 352 & 385 & 419 & 419 & 458 & 503 & 583 & 545 & 580 & : & 112 & 129 & 129 & 141 & 153 & 153 & 167 & 183 & 183 & 198 & 211\end{array}$ $\begin{array}{lllllllllllllllllllllll}332 & 361 & 383 & 490 & 425 & 445 & 465 & 491 & 569 & 536 & 554 & 128 & 139 & 147 & 154 & 162 & 169 & 176 & 185 & 192 & 281 & 288\end{array}$ $\begin{array}{lllllllllllllllllllllll}6 & 0 & 199 & 199 & 436 & 610 & 510 & 843 & 984 & 1230 & 1230 & : & 0 & 0 & 37 & 37 & 89 & 112 & 112 & 153 & 178 & 222 & 222\end{array}$

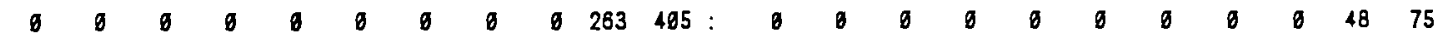
$\begin{array}{lllllllllllllllllllllll}545 & 580 & 580 & 614 & 660 & 697 & 697 & 746 & 773 & 773 & 865 & 225 & 239 & 239 & 253 & 272 & 287 & 287 & 387 & 318 & 318 & 331\end{array}$

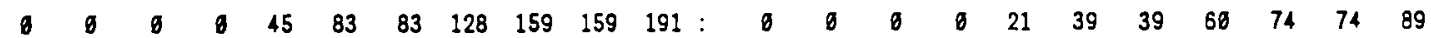

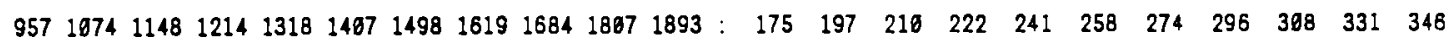




\section{TABLE A.4. Middle Case (with Extended Burnup) 1984 Projected Inventories (contd)}

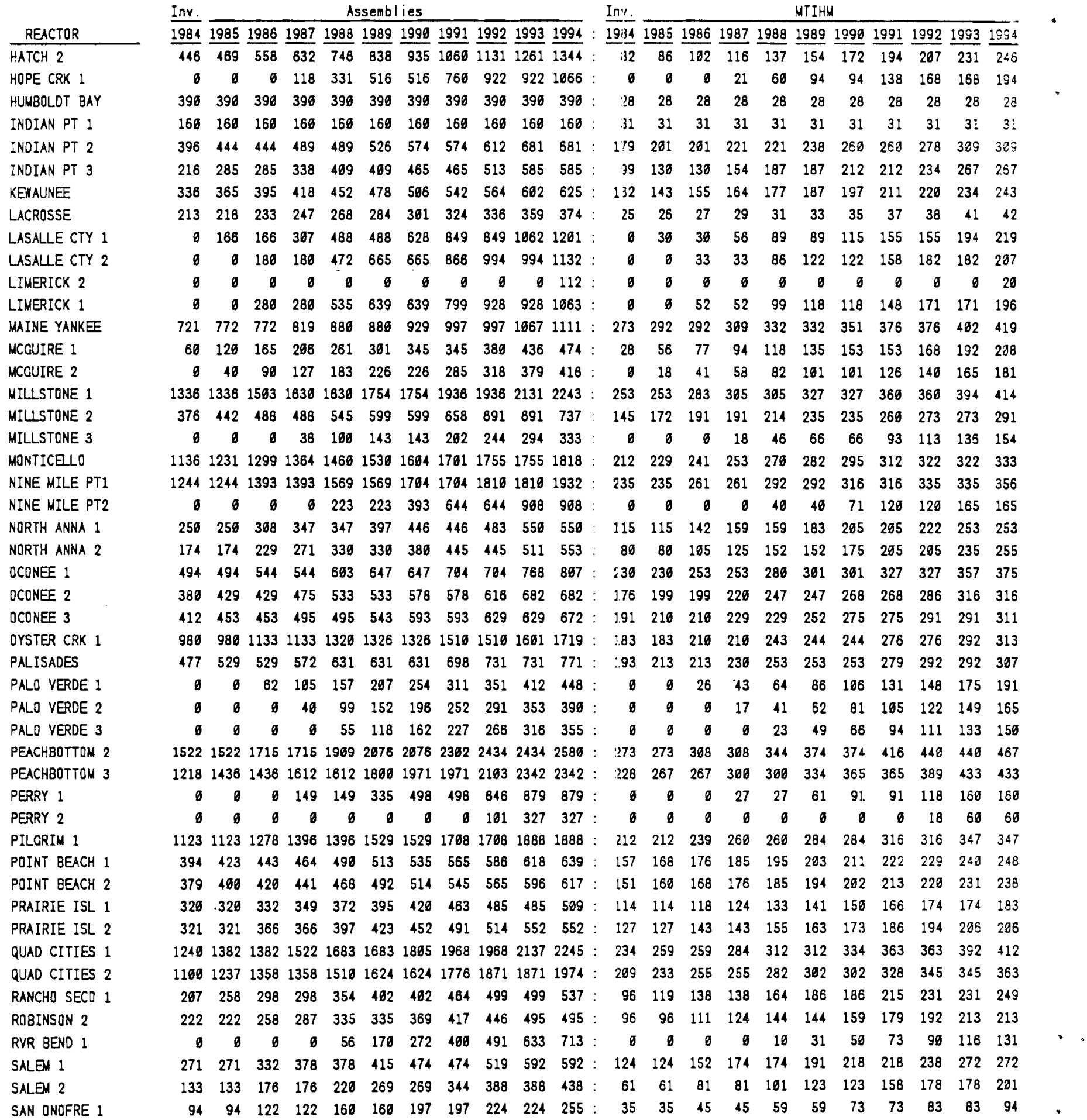


TABLE A.4. Middle Case (with Extended Burnup) 1984 Projected Inventories (contd)

\begin{tabular}{|c|c|c|c|c|c|c|c|c|c|c|c|c|c|c|c|c|c|c|c|c|c|c|}
\hline \multirow[b]{2}{*}{ REACTOR } & \multirow{2}{*}{$\frac{\text { Inv. }}{1984}$} & \multicolumn{10}{|c|}{ Assemblies } & \multirow{2}{*}{$\frac{\text { Inv. }}{1984}$} & \multicolumn{10}{|c|}{ MTIHM } \\
\hline & & $\underline{1985}$ & 1986 & 1987 & 1988 & 1989 & 1990 & 1991 & $\underline{1992}$ & 1993 & 94 & & 35 & 986 & 1987 & $\underline{1988}$ & $\underline{1989}$ & 1998 & 1991 & 1992 & 93 & 199 \\
\hline SAN ONOFRE 2 & 65 & 65 & 132 & 189 & 189 & 252 & 252 & 337 & 337 & 426 & 426 & 28 & 28 & 56 & 81 & 81 & 188 & 108 & 144 & 144 & 182 & 18 \\
\hline AN ONOFRE 3 & 8 & 46 & 11 & 11 & 181 & 181 & 248 & 248 & 360 & 300 & -7 & 0 & 20 & 47 & 47 & 77 & 77 & 86 & 36 & 28 & 28 & \\
\hline EABRODK 1 & 0 & ø & $\theta$ & 36 & 160 & 144 & 187 & 243 & 283 & 344 & 378 & 8 & & 0 & 15 & 42 & 61 & 79 & 63 & 28 & 146 & 16 \\
\hline$A B R O O K-2$ & 8 & 8 & $g$ & 8 & $\theta$ & 0 & 6 & 8 & 8 & 63 & Iac $\mathrm{r}$ & 8 & 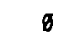 & $\theta$ & $\theta$ & 6 & 0 & $\theta$ & $\theta$ & $\theta$ & 27 & \\
\hline EQUOYAH 1 & 140 & 182 & 182 & 224 & 287 & 287 & 340 & 340 & 384 & 461 & 461 & 64 & 84 & 84 & 103 & 132 & 132 & 156 & 156 & 177 & 212 & 21 \\
\hline EQUCYAH 2 & 136 & 136 & 184 & 227 & 227 & 278 & 333 & 333 & 377 & 377 & 426 & 62 & 62 & 84 & 104 & 104 & 128 & 153 & 153 & 173 & 173 & $: 9$ \\
\hline YOREHAM & 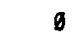 & $\emptyset$ & 0 & 142 & 142 & 286 & 452 & 452 & 528 & 662 & 2 & 8 & & $\theta$ & 26 & 26 & 52 & 33 & 83 & 97 & 121 & 12 \\
\hline OUTH TEXAS 1 & 8 & $\theta$ & $g$ & $\theta$ & 51 & 190 & 128 & 175 & 293 & 242 & 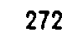 & 8 & 0 & 0 & 0 & 27 & 54 & 69 & 94 & 169 & 130 & 14 \\
\hline UUTH TEXAS 2 & $\theta$ & $\emptyset$ & $\emptyset$ & $\theta$ & 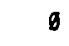 & 0 & $\emptyset$ & 35 & 63 & 110 & 1 & $\emptyset$ & 8 & $\emptyset$ & $\theta$ & $\emptyset$ & 6 & 8 & 19 & 34 & 59 & \\
\hline T LUCIE 1 & 372 & 445 & 445 & 492 & 572 & 572 & 627 & 716 & 716 & 793 & 855 & 142 & 170 & 170 & 187 & 211 & 211 & 232 & 258 & 258 & 287 & 36 \\
\hline T LUCIE 2 & 80 & 80 & 154 & 208 & 288 & 270 & 327 & 327 & 377 & 455 & 455 & 31 & 31 & 58 & 78 & 78 & 102 & 24 & 24 & 43 & 172 & 17 \\
\hline UMMER 1 & 44 & 141 & 141 & 193 & 248 & 298 & 345 & 345 & 385 & 447 & 92 & 20 & 65 & 65 & 89 & 114 & 37 & 59 & 59 & $m$ & 86 & 22 \\
\hline JRRY 1 & 477 & 477 & 529 & 567 & 567 & 689 & 653 & 653 & 687 & 744 & 744 & 217 & 217 & 240 & 258 & 258 & 77 & 97 & 97 & 13 & 39 & 33 \\
\hline JRRY 2 & 19 & 397 & 442 & 442 & 498 & 539 & 539 & 598 & 629 & 629 & 686 & 158 & 180 & 261 & 261 & 226 & 45 & 15 & 2 & $r$ & 3 & se \\
\hline JSQUEHANNA 1 & 8 & 158 & 328 & 484 & 484 & 650 & 817 & 817 & 948 & 1195 & 1195 & 9 & 29 & 60 & 89 & 89 & 18 & 48 & 8 & 71 & 14 & 21 \\
\hline USQUEHANNA 2 & $g$ & 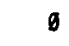 & 194 & 194 & 417 & 584 & 584 & 880 & 926 & 926 & 1 & $\theta$ & $a$ & 36 & 36 & 76 & 197 & 87 & 15 & 77 & 87 & 19 \\
\hline MILE ISL 1 & 268 & 208 & 298 & 261 & 321 & 321 & 371 & 436 & 436 & 87 & & 97 & 97 & 97 & 121 & 149 & 49 & 2 & 2 & 2 & 35 & 25 \\
\hline 20JAN & 3 & 369 & 346 & 381 & 422 & 454 & 482 & 519 & 542 & 580 & 6 & 130 & 142 & 159 & 175 & 194 & 69. & 221 & 39 & & 7 & 27 \\
\hline JRKEY PT 3 & 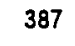 & 415 & 451 & 451 & 495 & 534 & 534 & 582 & 61 & 2 & & 175 & 88 & 265 & 205 & 225 & 43 & 243 & & & 79 & 29 \\
\hline URKEY PT 4 & 379 & 379 & 379 & 427 & 427 & 461 & 497 & 497 & 527 & 577 & 5 & 172 & 172 & 172 & 194 & 194 & 10 & 26 & 26 & 248 & 63 & 26 \\
\hline GTLE 1 & $\emptyset$ & 0 & 0 & 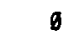 & 46 & 46 & 169 & 170 & 268 & 1 & & $\emptyset$ & & $\emptyset$ & 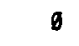 & 21 & 1 & & & & & 13 \\
\hline GTLE 2 & 8 & $\theta$ & $\theta$ & $\theta$ & $\theta$ & $\emptyset$ & 38 & 163 & 141 & 193 & 34 & 0 & 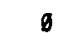 & 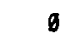 & $g$ & 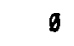 & 8 & 17 & 40 & 5 & 39 & 168 \\
\hline YANKEE 1 & 62 & 1268 & 1268 & 1339 & 1408 & 1469 & 1533 & 1618 & 1666 & 1753 & 1810 & 224 & 236 & 236 & 249 & 262 & 73 & 85 & 360 & 9 & 6 & 33 \\
\hline ISH NUCLEAR2 & $\emptyset$ & $\emptyset$ & 152 & 280 & 402 & 506 & 623 & 773 & 858 & 1011 & 1108 & $\theta$ & 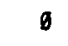 & 28 & 47 & 73 & 92 & 13 & 60 & 155 & 82 & 199 \\
\hline$P-1$ & $g$ & 9 & $a$ & $a$ & $\theta$ & $\theta$ & $g$ & b & 5 & & & $\theta$ & 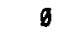 & 8 & $g$ & 8 & $g$ & $\theta$ & $a$ & - & 3 & 111 \\
\hline$P-3$ & 9 & 9 & 0 & 0 & 0 & 0 & $g$ & 78 & & & & $y$ & 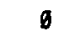 & 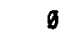 & $\nabla$ & 8 & 8 & $\theta$ & 政 & $\infty$ & 75 & 94 \\
\hline TERFORD 3 & $\theta$ & $a$ & 59 & 59 & 138 & 185 & 29 & 298 & & 6 & & $\emptyset$ & 8 & 25 & 25 & 56 & 76 & 95 & 123 & 3 & 53 & 170 \\
\hline ATTS BAR 1 & 6 & 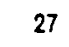 & 50 & 年 & .148 & 148 & 68 & & & & & ( & - & 43 & & 66 & 0 & & 0 & 110 & 110 & 138 \\
\hline ITTS BAR 2 & $\emptyset$ & $a$ & 9 & 35 & 195 & 195 & 149 & 1 & 208 & 36 & & $g$ & $g$ & 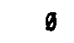 & 16 & 47 & 7 & 7 & 77 & 93 & 93 & 11 \\
\hline LF CRK 1 & $\theta$ & $\theta$ & $x^{2}+2$ & & B & 102 & 133 & & 286 & & & 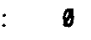 & 8 & $\emptyset$ & 1 & 31 & 47 & & & 15 & 6 & 127 \\
\hline ANKEE- $F$ & & 30 & 321 & 352 & 385 & 385 & & & 468 & & & 74 & 00 & 89 & 86 & 93 & 93 & 8 & 88 & 3 & 13 & 118 \\
\hline ron 1 & & & 495 & 527 & 569 & 692 & 635 & & 708 & 7 & & 197 & 210 & 226 & 246 & 268 & 275 & 38 & 18 & 22 & 13 & 356 \\
\hline ON 2 & & & & 532 & 574 & 607 & 041 & & 712 & & & 192 & 210 & 228 & 242 & 261 & 276 & 292 & 312 & 324 & 45 & 358 \\
\hline RUNSWICK-1 PWR P & 168 & 16 & 160 & 160 & 160 & 160 & 169 & 160 & 160 & & & 69 & & 0 & & 9 & 9 & 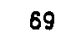 & 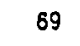 & 9 & 9 & 69 \\
\hline RUNSWICK-2 PWR POOL & $\mu$ & & $14+2>$ & 144 & 144 & 144 & 144 & 1 & 14 & 1 & & 65 & 65 & 65 & 65 & 65 & 65 & 65 & 65 & 65 & 65 & 6 \\
\hline MORRIS-BWR & 87 & 879 & 819 & $8 / 9$ & 879 & 879 & 87 & & & & & 107 & 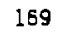 & 109 & 109 & 169 & 169 & 109 & 109 & 169 & 69 & 10 \\
\hline MORRIS-PWR & 350 & 350 & 350 & 358 & 350 & 358 & 350 & 350 & 350 & 358 & & 132 & 132 & 132 & 132 & 132 & 132 & 132 & 132 & 132 & 132 & 132 \\
\hline WEST VALLEY & 318 & 310 & 310 & 318 & 310 & 318 & 310 & 318 & 316 & 310 & 3 & 54 & 54 & 54 & 54 & 54 & 54 & 54 & 54 & 54 & 54 & 5 \\
\hline SSI VALLEY - & 121 & 121 & 121 & 121 & 121 & 121 & 121 & 121 & 121 & 121 & 181 & 40 & 46 & 46 & 46 & 46 & 46 & 46 & 46 & 46 & 46 & \\
\hline
\end{tabular}


TABLE A.4. Middle Case (with Extended Burnup) 1384 Projected Inventories

(contd)

REACTOR

ARK NUCLEAR 1

ARK NUCLEAR 2

B VALLEY 1

B VALLEY 2

BELLFONTE 1

9ELLEONTE 2

OIG ROCK 1

BRAIDYOOD 1

BRAIDYOOD 2

BROYNS FERRY1

BROWNS FERRY2

BROWNS FERRY3

BRUNSWICK 1

BRUNSWICK 2

BYRON 1

BYRON 2

CALLATAY 1

CALVERT CLF 1

CALVERT CLF 2

CARROL COUNTY-1

CARROLL COUNTY-2

CATAFEA 1.

CATANBA 2

CLINTON 1

COMANCHE PK 1

COMANCHE PK 2

COOK 1

$\operatorname{cook} 2$

COOPER STN

CRYSTAL RVR 3

DAVIS-BESSE 1

DIABLO CNYN 1

DIABLO CNYN 2

DRESDEN 1

DRESDEN 2

DRESDEN 3

DUANE ARNOLD

ENRICO FERMI2

FARLEY 1

FARLEY 2

FITZPATRICK

FORT CALHOUN

GINNA

GRAND GULF 1

GRAND GULF 2

HADDAM NECK

HARRIS 1

HATCH 1
Assemblies

$\frac{1995}{723} \frac{1996}{723} \frac{1997}{790} \frac{1998}{1999} \frac{2000}{80001} \frac{2002}{80003} \frac{2004}{004}$

$\begin{array}{cccccccccc}723 & 723 & 790 & 831 & 831 & 894 & 894 & 965 & 965 & 1019 \\ 576 & 576 & 650 & 702 & 782 & 776 & 770 & 845 & 845 & 961\end{array}$

$\begin{array}{llllllllll}595 & 637 & 637 & 682 & 735 & 735 & 772 & 850 & 850 & 909\end{array}$

$\begin{array}{llllllllll}248 & 248 & 367 & 358 & 358 & 421 & 459 & 459 & 499 & 566\end{array}$

$\begin{array}{llllllllll}181 & 219 & 219 & 292 & 345 & 345 & 396 & 486 & 488 & 558\end{array}$

$\begin{array}{llllllllll}124 & 178 & 170 & 222 & 222 & 291 & 342 & 342 & 393 & 478\end{array}$

$\begin{array}{lllllllllll}328 & 342 & 363 & 377 & 394 & 412 & 427 & 511 & 511 & 511 \text { : }\end{array}$

$\begin{array}{llllllllll}380 & 414 & 462 & 507 & 543 & 592 & 622 & 676 & 706 & 750 \text { : }\end{array}$

$\begin{array}{llllllllll}306 & 338 & 386 & 430 & 465 & 513 & 543 & 597 & 627 & 670\end{array}$

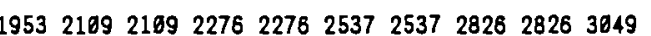

$22992299258925892788 \quad 2788 \quad 29502950 \quad 3108 \quad 3108$ :

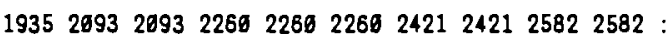

$\begin{array}{llllllllll}1346 & 1346 & 1530 & 1637 & 1637 & 1893 & 1896 & 1896 & 1994 & 2135\end{array}$

$16601751 \quad 1761 \quad 1887 \quad 199419942987227022702411$ :

$\begin{array}{llllllllll}437 & 478 & 519 & 564 & 599 & 648 & 677 & 731 & 761 & 895:\end{array}$

$\begin{array}{lllllllllll}381 & 413 & 460 & 504 & 539 & 587 & 617 & 671 & 781 & 745:\end{array}$

$\begin{array}{llllllllll}112 & 462 & 462 & 514 & 571 & 571 & 822 & 766 & 766 & 773 \text { : }\end{array}$

$\begin{array}{llllllllll}935 & 935 & 1012 & 1069 & 1080 & 1128 & 1178 & 1178 & 1228 & 1287\end{array}$ :

$\begin{array}{lllllllllll}829 & 868 & 939 & 939 & 992 & 1958 & 1958 & 1134 & 1182 & 1182 & \text { : }\end{array}$

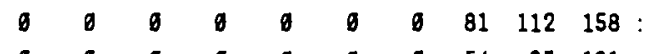

$\begin{array}{llllllllll}6 & 0 & 0 & 0 & 0 & 0 & 54 & 85 & 131\end{array}$

$\begin{array}{lllllllllll}418 & 453 & 503 & 553 & 590 & 640 & 677 & 731 & 731 & 781 & \text { : }\end{array}$

$\begin{array}{llllllllll}328 & 363 & 435 & 476 & 511 & 511 & 548 & 504 & 540 & 684:\end{array}$

$\begin{array}{llllllllll}910 & 1969 & 1198 & 1198 & 1345 & 1345 & 1459 & 1853 & 1653 & 1898 \text { : }\end{array}$

$\begin{array}{lllllllllll}439 & 479 & 532 & 583 & 623 & 678 & 719 & 780 & 819 & 869:\end{array}$

$\begin{array}{llllllllll}449 & 489 & 542 & 593 & 634 & 688 & 729 & 789 & 828 & 877:\end{array}$

$\begin{array}{llllllllllll}943 & 998 & 998 & 1044 & 1098 & 1698 & 1146 & 1146 & 1194 & 1259 & \text { : }\end{array}$

$\begin{array}{llllllllllll}753 & 753 & 842 & 898 & 898 & 1079 & 1970 & 1161 & 1217 & 1217\end{array}$

$1814 \quad 188220902069214922542322244125092596$ :

$\begin{array}{lllllllllll}553 & 587 & 587 & 621 & 680 & 689 & 695 & 753 & 753 & 797 & \text { : }\end{array}$ $\begin{array}{lllllllllll}544 & 583 & 583 & 623 & 671 & 871 & 798 & 779 & 779 & 832 & \text { : }\end{array}$

$\begin{array}{lllllllllll}399 & 438 & 582 & 592 & 546 & 619 & 643 & 843 & 676 & 731 & :\end{array}$

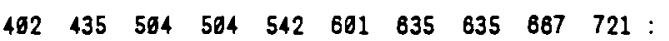
$\begin{array}{llllllllll}683 & 683 & 683 & 683 & 683 & 683 & 683 & 883 & 683 & 683\end{array}$ 2261237023702485262126212725292429243977 : $296620862229232423242471255725572843 \quad 2768$ : $\begin{array}{lllllllllll}1220 & 1286 & 1499 & 1469 & 1493 & 1603 & 1603 & 1726 & 1791 & 1791\end{array}$ $13851540154017651765 \quad 2014201422852443 \quad 2443$

$\begin{array}{llllllllll}704 & 794 & 776 & 822 & 822 & 885 & 928 & 928 & 967 & 1925\end{array}$ $\begin{array}{llllllllll}538 & 583 & 583 & 627 & 689 & 689 & 719 & 796 & 796 & 850\end{array}$

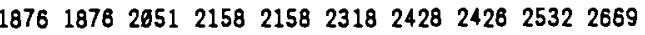
$\begin{array}{llllllllll}589 & 698 & 649 & 649 & 688 & 733 & 733 & 774 & 799 & 799\end{array}$ $\begin{array}{llllllllll}577 & 596 & 626 & 645 & 665 & 691 & 710 & 740 & 759 & 782\end{array}$ $\begin{array}{llllllllll}1448 & 1596 & 1596 & 1747 & 1936 & 1938 & 2985 & 2359 & 2562 & 2562\end{array}$ $\begin{array}{llllllllll}695 & 734 & 954 & 1112 & 1255 & 1469 & 1593 & 1812 & 1939 & 2125\end{array}$ $\begin{array}{lllllllllll}847 & 878 & 878 & 908 & 945 & 992 & 992 & 1047 & 1075 & 1075: \text { : }\end{array}$ $\begin{array}{llllllllll}244 & 244 & 288 & 288 & 321 & 373 & 373 & 422 & 455 & 455\end{array}$ 1997296922692282236924872551269127582859 :
MT IHM

$19951996 \quad 19971998199920002000120002 \frac{2003}{33} \underline{2004}$

$33 ; \quad 335 \quad \frac{366}{385} \frac{385}{414} \frac{114}{447} \frac{147}{472}$ $\begin{array}{llllllllll}241 & 241 & 272 & 293 & 293 & 322 & 322 & 353 & 353 & 376\end{array}$ $\begin{array}{llllllllll}274 & 293 & 293 & 314 & 338 & 338 & 355 & 391 & 391 & 418\end{array}$ $\begin{array}{llllllllll}114 & 114 & 142 & 165 & 165 & 194 & 212 & 212 & 236 & 261\end{array}$ $\begin{array}{llllllllll}81 & 98 & 98 & 131 & 156 & 156 & 179 & 220 & 220 & 253\end{array}$ $\begin{array}{llllllllll}54 & 75 & 75 & 98 & 98 & 136 & 153 & 153 & 176 & 215\end{array}$ $\begin{array}{llllllllll}43 & 44 & 47 & 49 & 51 & 53 & 55 & 66 & 66 & 66\end{array}$ $\begin{array}{llllllllll}161 & 175 & 195 & 214 & 236 & 250 & 263 & 286 & 298 & 317\end{array}$ $\begin{array}{llllllllll}129 & 143 & 163 & 182 & 197 & 217 & 230 & 252 & 265 & 283\end{array}$ $\begin{array}{llllllllll}362 & 390 & 390 & 421 & 421 & 468 & 468 & 520 & 520 & 561\end{array}$ $\begin{array}{llllllllll}425 & 425 & 478 & 478 & 514 & 514 & 543 & 543 & 572 & 572\end{array}$ $\begin{array}{lllllllllll}357 & 385 & 385 & 416 & 416 & 416 & 445 & 445 & 474 & 474\end{array}$ $\begin{array}{llllllllll}251 & 251 & 286 & 396 & 396 & 337 & 354 & 354 & 373 & 399\end{array}$ $\begin{array}{llllllllll}318 & 329 & 329 & 349 & 373 & 373 & 396 & 424 & 424 & 451\end{array}$ $\begin{array}{llllllllll}185 & 199 & 219 & 238 & 253 & 274 & 286 & 399 & 322 & 340\end{array}$ $\begin{array}{llllllllll}161 & 175 & 194 & 213 & 228 & 248 & 261 & 284 & 296 & 315\end{array}$ $\begin{array}{llllllllll}179 & 290 & 290 & 222 & 247 & 247 & 268 & 394 & 304 & 332\end{array}$ $\begin{array}{llllllllll}362 & 362 & 392 & 411 & 411 & 437 & 457 & 457 & 477 & 560\end{array}$ $\begin{array}{llllllllll}317 & 336 & 364 & 384 & 384 & 410 & 410 & 440 & 459 & 459\end{array}$ $\begin{array}{llllllllll}6 & 6 & 0 & 0 & 0 & 0 & 0 & 34 & 47 & 67\end{array}$ $\begin{array}{llllllllll}6 & 6 & 0 & 6 & 0 & 0 & 0 & 23 & 36 & 55\end{array}$ $\begin{array}{llllllllll}177 & 192 & 213 & 234 & 250 & 271 & 287 & 310 & 310 & 331\end{array}$ $\begin{array}{llllllllll}139 & 154 & 184 & 292 & 216 & 216 & 232 & 256 & 271 & 290\end{array}$ $\begin{array}{llllllllll}166 & 196 & 218 & 218 & 246 & 246 & 267 & 392 & 392 & 331\end{array}$ $\begin{array}{llllllllll}188 & 294 & 225 & 246 & 262 & 284 & 391 & 325 & 341 & 361\end{array}$ $\begin{array}{llllllllll}185 & 291 & 222 & 243 & 259 & 281 & 298 & 322 & 337 & 357\end{array}$ $\begin{array}{llllllllll}420 & 442 & 442 & 467 & 492 & 492 & 514 & 514 & 536 & 566\end{array}$ $\begin{array}{llllllllll}323 & 323 & 358 & 381 & 381 & 451 & 451 & 487 & 510 & 519\end{array}$ $\begin{array}{llllllllll}336 & 349 & 370 & 383 & 397 & 416 & 429 & 450 & 463 & 479\end{array}$ $\begin{array}{llllllllll}257 & 273 & 273 & 288 & 396 & 396 & 323 & 350 & 350 & 379\end{array}$ $\begin{array}{llllllllll}255 & 274 & 274 & 292 & 315 & 315 & 332 & 365 & 365 & 390\end{array}$ $\begin{array}{llllllllll}183 & 291 & 231 & 231 & 251 & 289 & 295 & 295 & 310 & 336\end{array}$ $\begin{array}{llllllllll}185 & 260 & 231 & 231 & 249 & 276 & 291 & 291 & 396 & 331\end{array}$ $\begin{array}{llllllllll}69 & 69 & 69 & 69 & 69 & 69 & 69 & 69 & 69 & 69\end{array}$ $\begin{array}{llllllllll}418 & 437 & 437 & 458 & 482 & 482 & 501 & 537 & 537 & 564\end{array}$ $\begin{array}{llllllllll}387 & 387 & 417 & 435 & 435 & 462 & 478 & 478 & 494 & 517\end{array}$ $\begin{array}{llllllllll}225 & 237 & 260 & 260 & 275 & 295 & 295 & 317 & 329 & 329\end{array}$ $\begin{array}{llllllllll}253 & 281 & 281 & 322 & 322 & 368 & 368 & 417 & 446 & 446\end{array}$ $\begin{array}{llllllllll}324 & 324 & 357 & 378 & 378 & 407 & 427 & 427 & 445 & 472\end{array}$ $\begin{array}{llllllllll}248 & 268 & 268 & 289 & 313 & 313 & 331 & 367 & 367 & 392\end{array}$ $\begin{array}{lllllllllll}346 & 346 & 377 & 396 & 396 & 425 & 445 & 445 & 464 & 488\end{array}$ $\begin{array}{llllllllll}211 & 221 & 235 & 235 & 249 & 265 & 265 & 280 & 289 & 289\end{array}$ $\begin{array}{llllllllll}216 & 222 & 233 & 239 & 246 & 255 & 262 & 273 & 279 & 287\end{array}$ $\begin{array}{llllllllll}260 & 286 & 286 & 313 & 346 & 346 & 372 & 421 & 446 & 446\end{array}$ $\begin{array}{llllllllll}111 & 135 & 176 & 295 & 231 & 279 & 293 & 333 & 357 & 391\end{array}$ $\begin{array}{llllllllll}349 & 361 & 361 & 374 & 389 & 468 & 468 & 431 & 442 & 442\end{array}$ $\begin{array}{llllllllll}113 & 113 & 134 & 134 & 149 & 173 & 173 & 196 & 212 & 212\end{array}$ $\begin{array}{llllllllll}365 & 378 & 492 & 417 & 433 & 455 & 466 & 492 & 594 & 522\end{array}$ 
TABLE A.4. Middle Case (with Extended Burnup) 1984 Projected Inventories (contd)

\begin{tabular}{|c|c|c|c|c|c|c|c|c|c|c|c|c|c|c|c|c|c|c|c|c|}
\hline & & & & & & & & & & & & & & & & & & & & \\
\hline REACTOR & $\underline{b}$. & 996 & 19 & $\underline{1998}$ & 9 & 2000 & 2001 & 2002 & 2603 & 2004 & 1995 & $\underline{1996}$ & $1997 \leq$ & $\underline{1998}:$ & $1999 ?$ & $2000 ?$ & 20812 & 2002 & 2003 & 2004 \\
\hline $\mathrm{TCH} 2$ & $\overline{1456}$ & $\overline{1532}$ & $\overline{1672}$ & $\overline{1753}$ & $\overline{1849}$ & $\overline{1974}$ & $\overline{2042}$ & $\overline{2184}$ & $\overline{2257}$ & $\overline{2365}$ & 267 & 280 & 366 & $\overline{321}$ & $\overline{338}$ & $\overline{361}$ & $\overline{373}$ & 399 & $\overline{413}$ & $\overline{432}$ \\
\hline PE CRK 1 & 7 & 1337 & 1565 & 1751 & 1751 & 2 & 2133 & 2133 & $2282^{\circ}$ & 2529 & 43 & 243 & 285 & 19 & 19 & 61 & 388 & 388 & 15 & 68 \\
\hline MBOLDT BA & 390 & 390 & 390 & 390 & 398 & 398 & 396 & 390 & 398 & 396 & 28 & 28 & 28 & 28 & 28 & 28 & 28 & 28 & 28 & 28 \\
\hline DIAN P & 68 & 160 & 160 & 160 & 160 & 168 & 168 & 160 & 160 & 160 & 31 & 31 & 31 & 31 & 31 & 31 & 31 & 31 & 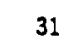 & . \\
\hline NDIAN PT 2 & 739 & 780 & 780 & 822 & 873 & 873 & 914 & 988 & 988 & 1045 & 336 & 355 & 355 & 374 & 397 & 397 & 416 & 450 & 150 & 476 \\
\hline NDIAN PT 3 & 649 & 649 & 728 & 783 & 783 & 851 & 851 & 946 & 983 & 983 & 296 & 296 & 332 & 357 & 357 & 388 & 388 & 429 & 149 & 149 \\
\hline SAU & 657 & 679 & 720 & 744 & 771 & 804 & 827 & 868 & 891 & 921 & 255 & 264 & 280 & 289 & 299 & 312 & 321 & 336 & 345 & 357 \\
\hline ICROSSE & 394 & 467 & 432 & 448 & 463 & 486 & 497 & 521 & 534 & 696 & 45 & 46 & 49 & 50 & 52 & 55 & 56 & 58 & 60 & 88 \\
\hline SALLE C & 1261 & 1327 & 1557 & 1557 & 1713 & 1928 & 1928 & 2150 & 2279 & 2279 & 219 & 242 & 284 & 284 & 312 & 350 & 350 & 392 & 416 & 416 \\
\hline ASALLE & 322 & 1322 & 1552 & 1685 & 1685 & 1893 & 2025 & 2025 & 2154 & 2330 & 241 & 241 & 283 & 308 & 68 & 346 & 370 & 370 & 93 & 425 \\
\hline IMERICK 2 & 112 & 365 & 520 & 520 & 792 & 1838 & 1636 & 1191 & 1384 & 1384 & 20 & 66 & 95 & 95 & 144 & 189 & 189 & 217 & 152 & 252 \\
\hline CK 1 & 282 & 1282 & 1468 & 1620 & 1620 & 1868 & & 1951 & 8 & 2290 & 236 & 236 & 276 & 297 & 297 & 32 & 58 & 58 & 83 & 28 \\
\hline MAINE YANK & 1111 & 1153 & 1227 & 1227 & 1278 & 1344 & 1344 & 1418 & 1461 & 1461 & 419 & 435 & 462 & 462 & 482 & 586 & 586 & 534 & 58 & 558 \\
\hline CGUIRE 1 & 528 & 561 & 623 & 862 & 764 & 784 & 746 & 605 & 841 & 887 & 230 & 245 & 271 & 288 & 365 & 385 & 321 & 348 & 363 & 383 \\
\hline CGUIRE 2 & $47 \theta$ & 594 & 569 & 569 & 813 & 670 & 767 & 769 & 865 & 852 & 204 & 218 & 248 & 246 & 265 & 289 & 394 & 331 & 46 & 366 \\
\hline MILLSTONE 1 & 2243 & 2353 & 2353 & 2474 & 2474 & 2642 & 2642 & 2850 & 2957 & 2957 & 414 & 434 & 434 & 455 & 455 & 485 & 485 & 522 & 541 & 541 \\
\hline IILLS & 88 & 788 & 855 & 904 & 984 & & 997 & 997 & & 1696 & 12 & 12 & 339 & 9 & 359 & 383 & 397 & 397 & 412 & 37 \\
\hline MILLSTONE 3 & 333 & 372 & 423 & 475 & 512 & 577 & 614 & 614 & 653 & 696 & 154 & 172 & 195 & 219 & 236 & 266 & 283 & 283 & 381 & 321 \\
\hline IONTICELIO & 1993 & 1961 & 2853 & 2853 & 2148 & 2231 & 2290 & 2397 & 2456 & 2538 & 348 & 358 & 374 & 374 & 391 & 466 & 416 & 435 & 46 & \\
\hline INE NILE & 132 & 2048 & 2048 & 2183 & 2163 & 2347 & 2347 & 2554 & 2554 & 2712 & 358 & 376 & 376 & 397 & 397 & 429 & 429 & 466 & 166 & 494 \\
\hline IINE MILE & 57 & 1157 & 1381 & 1565 & 1585 & 1792 & 1792 & 2042 & 2842 & 2247 & 211 & 211 & 251 & 284 & 284 & 25 & 325 & 376 & 70 & 187 \\
\hline IORTH & 687 & 645 & 645 & 687 & 736 & 736 & 775 & 847 & 847 & 901 & 279 & 297 & 297 & 316 & 339 & 339 & 357 & 396 & 96 & 415 \\
\hline YORTH ANMA 2 & 553 & 592 & 663 & 663 & 711 & 773 & 773 & 846 & 684 & 884 & 255 & 273 & 386 & 386 & 328 & 356 & 356 & 390 & 488 & 468 \\
\hline CONEE 1 & 897 & 845 & 913 & 913 & 958 & 958 & 998 & 1981 & 1961 & 1113 & 375 & 393 & 424 & 424 & 445 & 5 & 3 & 3 & 3 & 17 \\
\hline CONEE 2 & 739 & 778 & 778 & 818 & 818 & 884 & 884 & 951 & 989 & 989 & 343 & 361 & 361 & 379 & 379 & 410 & 410 & 441 & 459 & 459 \\
\hline CONEE 3 & 728 & 728 & 880 & 800 & 847 & 847 & 885 & 957 & 957 & 1011 & 337 & 337 & 371 & 371 & 92 & 32 & 410 & 443 & 443 & 469 \\
\hline YSTER CRK & 19 & 1780 & 1760 & 1857 & 1857 & 2 & 21 & 2119 & 2217 & 2217 & 313 & 328 & 328 & 18 & 38 & 35 & 384 & 384 & 401 & 401 \\
\hline ALISADES & 35 & 869 & 869 & 910 & 955 & 955 & 989 & 1655 & 1055 & 1168 & 332 & 345 & 345 & 361 & 379 & 79 & 392 & 418 & 18 & 38 \\
\hline ALO VERDE 1 & 510 & 549 & 694 & 656 & 697 & 753 & 792 & 854 & 892 & 942 & 218 & 235 & 259 & 281 & 299 & 324 & 341 & 368 & 384 & 66 \\
\hline ALO VERDE 2 & 452 & 491 & 548 & 598 & 639 & 695 & 734 & 795 & 833 & 883 & 192 & 289 & 233 & 258 & 274 & 298 & 315 & 342 & 58 & 380 \\
\hline ALO VERDE 3 & 418 & 455 & 525 & 569 & 689 & 675 & 714 & 773 & 811 & 870 & 177 & 193 & 224 & 243 & 261 & 289 & 306 & 332 & 349 & 375 \\
\hline EACHBOTT & 2780 & 2780 & 3927 & 3171 & 3171 & 3391 & 3534 & 3534 & 3679 & 3867 & 584 & 594 & 550 & 577 & 577 & 617 & 644 & 644 & 671 & 765 \\
\hline EACHBOTTOM 3 & 48 & 2691 & 2891 & 2839 & 3914 & 3014 & 3156 & 3414 & 34 & 3694 & 470 & 496 & 498 & 523 & 555 & 555 & 581 & 628 & 628 & 663 \\
\hline ERRY 1 & 4 & 1257 & 1 & 1448 & 1 & & 17 & 1970 & & 2157 & 283 & 229 & 229 & 34 & 32 & 22 & 17 & 9 & 59 & 93 \\
\hline RRY 2 & & 885 & 865 & 1022 & 1217 & 1217 & 1376 & 1674 & 1674 & 1925 & 115 & 147 & 147 & 186 & 222 & 22 & 251 & 305 & 95 & 51 \\
\hline LGRIM 1 & 45 & 2151 & 2151 & 2286 & 2266 & 2446 & 2446 & 2641 & 2641 & 2795 & 375 & 394 & 394 & 414 & 414 & 446 & 446 & 481 & 481 & 588 \\
\hline INT BEACH & 665 & 687 & 721 & 743 & 765 & 793 & 815 & 850 & 872 & 898 & 257 & 265 & 277 & 285 & 292 & 302 & 310 & 323 & 39 & 348 \\
\hline INT BEACH 2 & 644 & 666 & 699 & 721 & 744 & 774 & 796 & 836 & 852 & 878 & 248 & 256 & 267 & 275 & 283 & 94 & 382 & 314 & 21 & 31 \\
\hline AIRIE ISL 1 & 541 & 566 & 081 & 632 & 632 & 659 & 694 & 735 & 106 & 791 & 195 & 204 & 219 & 228 & 228 & 241 & 251 & 6 & 5 & 286 \\
\hline AIRIE ISL 2 & 585 & 618 & 651 & 676 & 784 & 742 & 742 & 783 & 868 & 839 & 217 & 226 & 239 & 248 & 257 & 270 & 278 & 283 & 92 & 362 \\
\hline AD CITIES 1 & 45 & 2345 & 2527 & 2527 & 2650 & 2813 & 2813 & 2993 & 3094 & 3094 & 412 & 429 & 461 & 461 & 483 & 512 & 512 & 543 & 61 & 561 \\
\hline AD CITIES 2 & 2169 & 2169 & 2282 & 2384 & 2384 & 2540 & 2644 & 2644 & 2746 & 2878 & 387 & 387 & 418 & 436 & 436 & 463 & 481 & 81 & 99 & 23 \\
\hline NCHO SECO 1 & 587 & 587 & 648 & 685 & 685 & 746 & 777 & 777 & 814 & 862 & 272 & 272 & 308 & 317 & 317 & 342 & 359 & 359 & 77 & 399 \\
\hline BINSON 2 & & 567 & 621 & 652 & 652 & 786 & 731 & 784 & 814 & 814 & 231 & 244 & 267 & 280 & 280 & 301 & 314 & 337 & 358 & 50 \\
\hline P BEND 1 & 57 & 946 & 1674 & 1193 & 1286 & 1417 & 1501 & 1644 & 1728 & 1840 & 57 & 173 & 196 & 218 & 235 & 259 & 274 & 360 & 315 & 336 \\
\hline LEN 1 & 3 & 700 & 780 & 747 & 880 & 800 & 847 & 925 & 925 & 985 & 300 & 321 & 321 & 343 & 367 & 367 & 389 & 425 & 25 & 152 \\
\hline EN 2 & 501 & 501 & 578 & 625 & 625 & 690 & 743 & 743 & 196 & 845 & 238 & 238 & 265 & 287 & 287 & 320 & 341 & 341 & 363 & 396 \\
\hline W ONOFF & 255 & 284 & 284 & 317 & 383 & 520 & 520 & 520 & 520 & 520 & 94 & 165 & 165 & 117 & 134 & 193 & 193 & 193 & 193 & 193 \\
\hline
\end{tabular}


TABLE A.4. Middle Case (with Extended Burnup) 1984 Projected Inventories (contd)

\begin{tabular}{|c|c|c|c|c|c|c|c|c|c|c|c|c|c|c|c|c|c|c|c|c|}
\hline & & & & & & & & & & 184 & & & & & & 60 & 2001 & $=$ & $\underline{2}$ & 2 \\
\hline FRE 2 & 500 & 500 & 590 & 590 & 53 & 653 & 711 & 711 & 770 & 840 & 213 & 213 & 252 & 252 & 279 & 279 & 304 & 304 & 29 & 59 \\
\hline E 3 & 7 & 14 & 507 & 17 & 3 & 73 & 631 & 31 & 690 & 690 & 2 & 77 & 16 & 16 & 45 & 45 & 69 & 69 & 9 & 95 \\
\hline ABROOK & 10 & 477 & 531 & 82 & 2 & 77 & 710 & 770 & 804 & 8 & 86 & 02 & 225 & 16 & 53 & 37 & & 326 & 40 & 61 \\
\hline$A B R$ & 67 & 204 & 271 & 18 & 360 & 424 & 468 & 525 & 562 & 618 & 71 & 86 & 115 & 35 & 52 & 38 & 195 & 22 & 38 & 262 \\
\hline Quo & 5 & 572 & 572 & 21 & 1 & 694 & 742 & 742 & 1 & 853 & 241 & 263 & 263 & 35 & 35 & 19 & 11 & 341 & 00 & 92 \\
\hline QUOYAH 2 & 491 & 491 & 573 & 573 & 630 & 704 & 704 & 785 & 833 & 833 & 226 & 226 & 263 & 63 & 289 & 23 & 23 & 361 & 383 & 83 \\
\hline ORE & 1 & 846 & 846 & 46 & $\theta$ & 930 & 1014 & 1022 & 1022 & 1154 & 139 & 154 & 154 & 154 & 176 & 70 & 85 & 87 & 87 & 211 \\
\hline UTH & 29 & 346 & 400 & 434 & 461 & 510 & 536 & 581 & 607 & 650 & 172 & 185 & 215 & 234 & 248 & 75 & 289 & 313 & 327 & 350 \\
\hline XAS 2 & 88 & 216 & 264 & 391 & 332 & 380 & 469 & 457 & 485 & 527 & 101 & 116 & 142 & 162 & 179 & 205 & 228 & 246 & 261 & 284 \\
\hline LUC & 24 & 924 & 1020 & 1072 & 1072 & 1153 & 1295 & 1295 & 1269 & 1332 & 332 & 332 & 361 & 389 & 189 & (200 & 425 & 425 & 45 & 469 \\
\hline LUCIE 2 & 21 & 573 & 573 & 628 & 84 & 684 & 736 & 820 & 820 & 883 & 97 & 217 & 217 & 37 & 59 & 59 & 279 & 11 & 11 & 35 \\
\hline MME & 4 & 44 & 619 & 57 & & 766 & 768 & 842 & & 932 & 0 & 50 & 285 & 22 & & & & 87 & 03 & 28 \\
\hline RRY 1 & 95 & 830 & 830 & 867 & 88 & 908 & 944 & 1087 & 1007 & 1955 & 362 & 378 & 378 & 395 & 14 & 14 & 31 & 68 & 60 & 82 \\
\hline RRY 2 & 17 & 717 & 781 & 816 & 816 & 873 & 968 & 998 & 944 & 990 & 327 & 327 & 356 & 372 & 372 & 199 & 15 & 15 & 131 & 452 \\
\hline SQUE & 74 & 1584 & 1594 & 1645 & 1812 & 1812 & 1950 & 2195 & 2195 & 2383 & 245 & 268 & 268 & 293 & 22 & 22 & 46 & 89 & 89 & 422 \\
\hline ISQUE & 1268 & 1268 & 1512 & 1854 & 1654 & 1876 & 2013 & 2013 & 2147 & 2337 & 228 & 228 & 271 & 298 & 95 & 35 & 359 & 59 & 38 & 416 \\
\hline MILE & 2 & 94 & 670 & 670 & 722 & 790 & 790 & 866 & & 967 & 56 & 6 & 311 & 11 & & & 365 & 02 & 421 & 21 \\
\hline 10 JAN & 37 & 861 & 703 & 727 & 55 & 791 & 816 & 858 & 882 & 914 & 293 & 304 & 323 & 335 & 47 & 34 & 376 & 95 & 486 & 421 \\
\hline RKEY & 87 & 687 & 740 & 73 & 73 & 819 & 852 & 852 & 8 & 92 & 313 & 13 & 337 & 52 & 352 & $3 / 4$ & 89 & 89 & 494 & 423 \\
\hline IRKEY & 21 & 653 & 653 & 686 & 23 & 723 & 756 & 899 & 899 & 85 & 283 & 298 & 298 & 13 & 30 & 30 & 345 & 699 & 369 & 388 \\
\hline GTLE 1 & 367 & 401 & 473 & 519 & 56 & 623 & 658 & 718 & 752 & 81 & 169 & 185 & 218 & 39 & & 37 & 384 & 331 & 47 & 74 \\
\hline GTLE 2 & 297 & 331 & 403 & 448 & 84 & 549 & 584 & 643 & 17 & 73 & 37 & 53 & 86 & 87 & & 53 & 69 & 97 & 112 & 39 \\
\hline Y YANKEE 1 & 1885 & 1936 & 2631 & 2084 & 42148 & 2231 & 2281 & 2375 & 2425 & 2496 & 349 & 358 & 376 & 385 & 97 & 412 & 421 & 38 & 48 & 61 \\
\hline ASH N & 1240 & 1330 & 1497 & 1593 & 31705 & 1852 & 1945 & 2110 & 2201 & 2329 & 22 & 38 & 67 & 84 & & 30 & 46 & 75 & 991 & 14 \\
\hline$P-1$ & 37 & 345 & 412 & 459 & $9 \quad 501$ & 565 & 601 & 666 & 793 & 758 & 140 & 157 & 188 & 289 & 29 & 58 & 274 & 94 & 21 & 146 \\
\hline P-3 & & & 468 & 459 & 0 & 576 & 611 & 681 & 72 & 78 & 123 & 141 & 173 & 195 & 215 & 240 & 68 & 98 & 308 & 333 \\
\hline ITERFORD 3 & 75 & 589 & 569 & 570 & 66 & 678 & 713 & 713 & 782 & 82 & 200 & 214 & 214 & 240 & 256 & 286 & 391 & 361 & 322 & 347 \\
\hline ITS & & 362 & 436 & 484 & 4 & 552 & 552 & 629 & 870 & 67 & 85 & 165 & 196 & 21 & 21 & 52 & 252 & & 309 & 99 \\
\hline ATSS BAR 2 & & 290 & 377 & 377 & 7426 & 597 & 597 & 588 & 580 & 64 & 11 & 132 & 172 & 172 & 94 & 31 & 231 & 265 & 265 & 293 \\
\hline JLF CRK 1 & 22 & 351 & 392 & 430 & 461 & 581 & 527 & 572 & 598 & 63 & 49 & 182 & 181 & 198 & 3 & 31 & ?43 & 64 & 76 & 293 \\
\hline ANKEE- & & 524 & 567 & 643 & 3643 & 643 & 643 & 643 & 643 & 64 & 126 & 126 & 136 & 154 & 154 & 154 & 154 & 154 & 154 & 154 \\
\hline ON 1 & & 7 & 6 & 925 & & 1804 & 1032 & 1683 & 1116 & 1148 & 74 & 387 & 409 & 422 & 37 & 58 & 71 & 95 & 07 & 24 \\
\hline ION 2 & & 854 & 984 & 933 & $\begin{array}{ll}3 & 967\end{array}$ & 1811 & 1939 & 1996 & 1117 & 1155 & 377 & 389 & 412 & 425 & 441 & 461 & 474 & 497 & 509 & 527 \\
\hline RUNSWICK-1 PWR & 180 & 160 & 160 & 189 & 168 & 168 & 169 & 180 & 160 & 16 & 69 & 69 & 69 & 69 & & 09 & 69 & 39 & 69 & 69 \\
\hline RUNSWICK-2 PWR POOL & 144 & 144 & 144 & 144 & $4 \quad 144$ & 144 & 144 & 144 & 144 & 14 & 65 & 65 & 65 & 65 & bo & 65 & 65 & 65 & 65 & 65 \\
\hline RRIS-BW & 87 & 879 & 879 & 879 & 9879 & 879 & 879 & 879 & 9 & 87 & 169 & 169 & 169 & 169 & 169 & 169 & 169 & 169 & 169 & 169 \\
\hline RRIS-PWR & 350 & 350 & 350 & 350 & 58 & 350 & 350 & 350 & 350 & 35 & 132 & 132 & 132 & 132 & 132 & 132 & 132 & 132 & 132 & 132 \\
\hline EST VALLEY & 318 & 310 & 310 & 310 & 310 & 310 & 310 & 310 & 310 & 31 & 54 & 54 & 54 & 54 & 54 & 54 & 54 & 54 & 54 & 54 \\
\hline ST VALLEY & 21 & 121 & 121 & 121 & 21 & 121 & 121 & 121 & 121 & 12 & 46 & 46 & 46 & 46 & 46 & 46 & 46 & 46 & 46 & 46 \\
\hline ENERIC PWR & 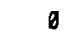 & $g$ & $g$ & $\theta$ & 8 & 8 & a & 6 & 124 & 566 & $\theta$ & $\theta$ & $\theta$ & 0 & 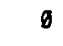 & 0 & (t) & 8 & 57 & 261 \\
\hline ENERIC BWR & 0 & 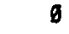 & 9 & 9 & 8 & 9 & $\varnothing$ & 6 & 85 & 960 & 8 & 8 & 6 & $\theta$ & 0 & 0 & $\theta$ & $\theta$ & 52 & 176 \\
\hline
\end{tabular}


TABLE A.4. Middle Case (with Extended Burnup) 1984 Projected Inventories (contd)

\begin{tabular}{|c|}
\hline REACTOR \\
\hline ARK NUCLEAR 1 \\
\hline ARK NUCLEAR 2 \\
\hline B VALLEY 1 \\
\hline B VALLEY 2 \\
\hline BELEFONTE 1 \\
\hline 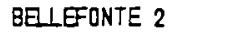 \\
\hline EIG ROCK 1 \\
\hline BRAIDWOOD 1 \\
\hline BRAIDWOOD 2 \\
\hline BROWNS FERRY1 \\
\hline BROHNS FERRY2 \\
\hline BROWNS FERRY3 \\
\hline BRUNSWICX 1 \\
\hline BRUNSWICX 2 \\
\hline BYRON 1 \\
\hline EYRON 2 \\
\hline CALLAMAY 1 \\
\hline CALVERT CLF 1 \\
\hline CALYERT CLF 2 \\
\hline CARROL COUNTY-1 \\
\hline CARROLL COUNTY-2 \\
\hline CATAMBA 1 \\
\hline CATATEA 2 \\
\hline CLINTON 1 \\
\hline COMANCHE PX 1 \\
\hline $\begin{array}{l}\text { COMANCHE PK } 2 \\
\text { COOK } 1\end{array}$ \\
\hline
\end{tabular}

$\operatorname{cook} 2$

COOPER STN

CRYSTAL RVR 3

DAVIS-BESSE 1

DIABLO CNYN 1

DIABLO CNYN 2

DRESDEN 1

DRESDEN 2

DRESDEN 3

DUANE ARNOLO

ENRICO FERMI2

FARLEY 1

FARLEY 2

FITZPATRICK

FORT CALHOUN

GINMA

GRAND GLUF 1

GRAND GUF 2

HADOAM NECX

HARRIS 1

HATCH 1
Assemblies

$\frac{2005}{1064} \frac{2008}{1064} \frac{2007}{1105} \frac{2008}{1105} \frac{2009}{1162} \frac{2010}{1339} \frac{2011}{1339} \frac{2012}{1339} \frac{2013}{1339} \frac{2014}{1339}: \overline{\frac{2005}{493} \frac{2006}{493} \frac{2007}{512} \frac{2008}{512} \frac{2009}{538} \frac{2010}{621} \frac{2011}{621} \frac{2912}{621} \frac{2013}{621} \frac{2014}{621}}$

$\begin{array}{lllllllllllllllllllll}950 & 950 & 999 & 999 & 1059 & 1859 & 1135 & 1194 & 1371 & 1371 & : & 397 & 397 & 417 & 417 & 442 & 442 & 474 & 498 & 572 & 572\end{array}$

$\begin{array}{llllllllllllllllllllll}957 & 957 & 1080 & 1036 & 1036 & 1872 & 1147 & 1147 & 1280 & 1252 & : & 441 & 441 & 460 & 477 & 477 & 494 & 528 & 528 & 553 & 577\end{array}$

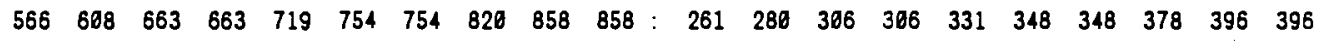

$\begin{array}{lllllllllllllllllllll}616 & 616 & 685 & 736 & 736 & 781 & 781 & 869 & 918 & 918 & : & 279 & 279 & 311 & 334 & 334 & 354 & 354 & 395 & 417 & 417\end{array}$

$\begin{array}{lllllllllllllllllllll}478 & 545 & 614 & 614 & 768 & 754 & 754 & 853 & 962 & 902 & : & 215 & 246 & 277 & 277 & 328 & 341 & 341 & 386 & 408 & 468\end{array}$

$\begin{array}{lllllllllllllllllllll}511 & 511 & 511 & 511 & 511 & 511 & 511 & 511 & 511 & 511 & 6 & 66 & 66 & 66 & 66 & 66 & 66 & 66 & 66 & 66 & 66\end{array}$

$\begin{array}{lllllllllllllllllllll}795 & 827 & 867 & 897 & 946 & 972 & 1609 & 1659 & 1107 & 1137 & : & 336 & 350 & 367 & 379 & 397 & 411 & 427 & 448 & 468 & 481\end{array}$

$\begin{array}{lllllllllllllllllllll}714 & 745 & 785 & 815 & 858 & 890 & 926 & 977 & 1025 & 1055 & : & 302 & 315 & 332 & 345 & 363 & 376 & 392 & 413 & 433 & 446\end{array}$

$\begin{array}{lllllllllllllllllllll}3649 & 3255 & 3255 & 3413 & 3413 & 4177 & 4177 & 4177 & 4177 & 4177 & : & 561 & 598 & 598 & 627 & 627 & 766 & 768 & 766 & 766 & 766\end{array}$

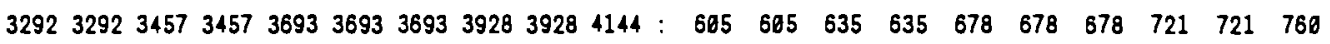

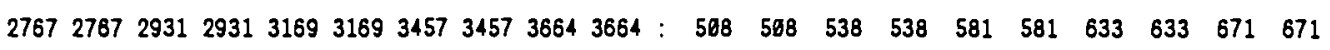

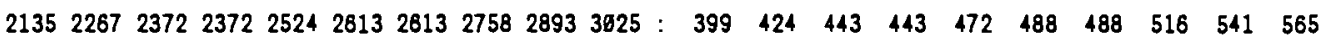

$\begin{array}{lllllllllllllllllllll}2528 & 2528 & 2632 & 2723 & 2723 & 2812 & 2989 & 2989 & 3118 & 3245 & : & 473 & 473 & 492 & 589 & 509 & 528 & 559 & 559 & 583 & 607\end{array}$

$\begin{array}{lllllllllllllllllllll}849 & 881 & 921 & 949 & 991 & 1623 & 1659 & 1109 & 1156 & 1182 & : & 359 & 372 & 389 & 461 & 419 & 432 & 448 & 469 & 489 & 560\end{array}$

$\begin{array}{lllllllllllllllllllll}789 & 821 & 881 & 891 & 934 & 986 & 1601 & 1652 & 1699 & 1129 & : & 334 & 347 & 364 & 377 & 395 & 488 & 423 & 445 & 465 & 477\end{array}$

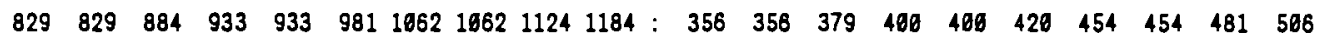

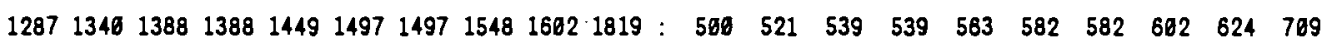
$\begin{array}{lllllllllllllllllllll}1238 & 1284 & 1284 & 1332 & 1392 & 1392 & 1468 & 1519 & 1519 & 1738 & : & 478 & 499 & 499 & 518 & 542 & 542 & 571 & 591 & 591 & 615\end{array}$ $\begin{array}{lllllllllllllllllllll}197 & 233 & 271 & 388 & 342 & 371 & 415 & 471 & 517 & 558 & : & 83 & 98 & 115 & 127 & 145 & 157 & 175 & 199 & 218 & 236\end{array}$ $\begin{array}{lllllllllllllllllllll}170 & 206 & 244 & 273 & 315 & 344 & 388 & 444 & 496 & 531 & : & 72 & 87 & 103 & 115 & 133 & 145 & 164 & 188 & 207 & 224\end{array}$ $\begin{array}{lllllllllllllllllllll}825 & 863 & 965 & 941 & 986 & 1019 & 1019 & 1073 & 1127 & 1158 & : & 349 & 366 & 383 & 399 & 418 & 432 & 432 & 455 & 477 & 491\end{array}$ $\begin{array}{llllllllllllllllllllll}733 & 774 & 819 & 819 & 863 & 899 & 939 & 998 & 1641 & 1671 & : & 311 & 328 & 347 & 347 & 366 & 381 & 398 & 419 & 441 & 454\end{array}$

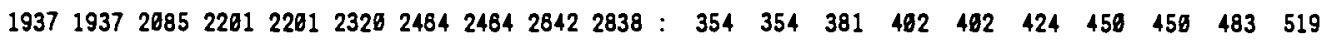
$\begin{array}{lllllllllllllllllllll}921 & 959 & 1005 & 1045 & 1094 & 1132 & 1174 & 1232 & 1283 & 1318 & : & 382 & 397 & 416 & 432 & 452 & 467 & 484 & 568 & 528 & 542\end{array}$

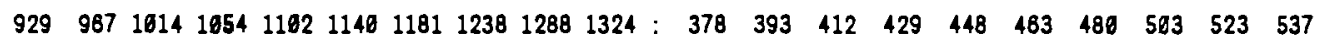

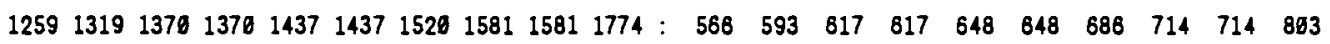

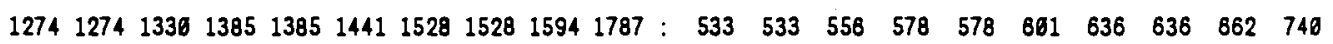

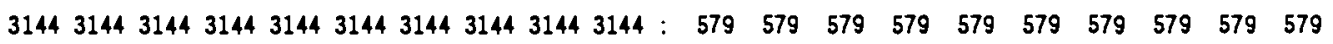
$\begin{array}{lllllllllllllllllllll}832 & 832 & 866 & 960 & 960 & 934 & 989 & 989 & 1629 & 1669 & : & 386 & 386 & 482 & 418 & 418 & 433 & 459 & 459 & 478 & 496\end{array}$ $\begin{array}{llllllllllllllllllllll}876 & 876 & 915 & 952 & 952 & 988 & 1657 & 1057 & 1107 & 1156 & : & 411 & 411 & 429 & 446 & 446 & 463 & 496 & 496 & 519 & 542\end{array}$ $\begin{array}{llllllllllllllllllllll}731 & 777 & 814 & 846 & 846 & 883 & 947 & 994 & 1040 & 1046 & : & 336 & 357 & 374 & 389 & 389 & 466 & 435 & 456 & 478 & 478\end{array}$ $\begin{array}{lllllllllllllllllllll}757 & 863 & 883 & 834 & 887 & 917 & 917 & 975 & 1625 & 1091 & : & 347 & 369 & 369 & 383 & 497 & 421 & 421 & 448 & 470 & 501\end{array}$ $\begin{array}{lllllllllllllllllllll}683 & 683 & 683 & 683 & 683 & 683 & 683 & 683 & 683 & 683 & : & 69 & 69 & 69 & 69 & 69 & 89 & 69 & 69 & 69 & 69\end{array}$

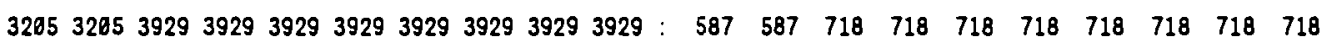
$\begin{array}{lllllllllllllllllllll}2768 & 2884 & 2977 & 3701 & 3701 & 3701 & 3701 & 3701 & 3701 & 3701 & : & 517 & 538 & 555 & 689 & 689 & 689 & 689 & 689 & 689 & 689\end{array}$

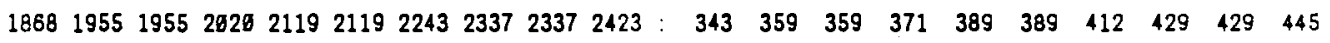
$\begin{array}{lllllllllllllllllllll}2667 & 2867 & 2871 & 2871 & 3895 & 3242 & 3242 & 3581 & 3501 & 3653 & : & 486 & 486 & 524 & 524 & 564 & 591 & 591 & 638 & 638 & 666\end{array}$

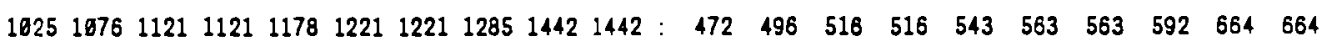
$\begin{array}{llllllllllllllllllllll}960 & 906 & 940 & 984 & 984 & 1022 & 1096 & 1096 & 1145 & 1201 & : & 415 & 415 & 433 & 453 & 453 & 471 & 505 & 585 & 528 & 554\end{array}$

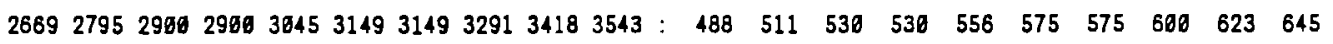

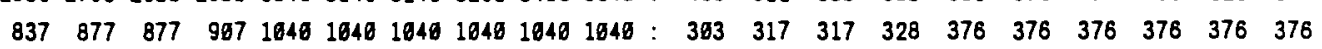
$\begin{array}{lllllllllllllllllllll}801 & 822 & 943 & 943 & 943 & 943 & 943 & 943 & 943 & 943 & : & 294 & 301 & 344 & 344 & 344 & 344 & 344 & 344 & 344 & 344\end{array}$

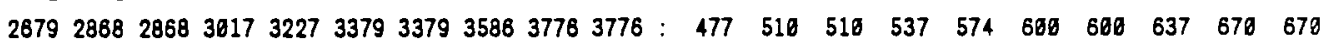

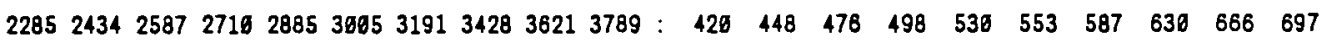
$\begin{array}{lllllllllllllllllllll}1110 & 1158 & 1307 & 1397 & 1397 & 1397 & 1307 & 1397 & 1387 & 1397 & : & 457 & 473 & 536 & 538 & 538 & 538 & 538 & 538 & 538 & 538\end{array}$ $\begin{array}{lllllllllllllllllllll}495 & 495 & 531 & 564 & 564 & 595 & 630 & 630 & 874 & 721 & 230 & 230 & 247 & 262 & 262 & 277 & 293 & 293 & 313 & 335\end{array}$

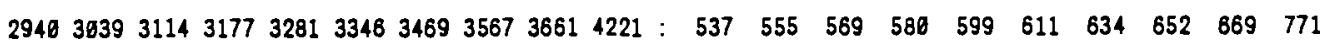


TABLE A.4. Middle Case (with Extended Burnup) 1984 Projected Inventories

REACTOR
HATCH 2
HOPE CRK 1
HUMBOLDT BAY
INDIAN PT 1
INDIAN PT 2
INDIAN PT 3
KENAUNEE
LACROSSE
LASALLE CTY 1
LASALLE CTY 2
LIMERICK 2
LIMERICX 1
MAINE YANKEE
MCCUIRE 1
MCGUIRE 2
MILLSTONE 1
MIULSTONE 2
MILSTIONE 3
MONTICELL
NINE MILE PT1
NINE MILE PT2
NORTH ANNA 1
NORTH ANNA 2
OCONEE 1
MONE

$\frac{2005}{2454} \frac{2006}{2554} \frac{2007}{2632} \frac{2008}{2699} \frac{2099}{2812} \frac{2016}{2877} \frac{2011}{3018} \frac{2012}{3122} \frac{2013}{3221} \frac{2014}{3781}:$
252926832883

MTIHM

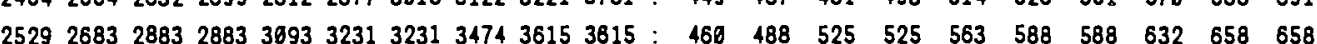
$\begin{array}{lllllllllllllllllllll}390 & 390 & 398 & 390 & 398 & 398 & 398 & 398 & 390 & 390 & : & 28 & 28 & 28 & 28 & 28 & 28 & 28 & 28 & 28 & 28\end{array}$ $\begin{array}{lllllllllllllllllllll}160 & 160 & 160 & 160 & 160 & 160 & 160 & 160 & 160 & 160 & : & 31 & 31 & 31 & 31 & 31 & 31 & 31 & 31 & 31 & 31\end{array}$ $\begin{array}{lllllllllllllllllllll}1693 & 1093 & 1135 & 1178 & 1176 & 1369 & 1369 & 1369 & 1369 & 1369 & : & 498 & 498 & 518 & 537 & 537 & 625 & 625 & 625 & 625 & 625\end{array}$ $\begin{array}{lllllllllllllllllllll}1038 & 1038 & 1093 & 1143 & 1143 & 1192 & 1192 & 1251 & 1305 & 1395 & : & 474 & 474 & 499 & 521 & 521 & 544 & 544 & 571 & 595 & 595\end{array}$ $\begin{array}{llllllllllllllllllllll}946 & 974 & 996 & 1019 & 1050 & 1171 & 1171 & 1171 & 1171 & 1171 & : & 366 & 377 & 386 & 394 & 496 & 453 & 453 & 453 & 453 & 453\end{array}$ $\begin{array}{lllllllllllllllllllll}606 & 606 & 606 & 606 & 606 & 606 & 606 & 606 & 606 & 608 & : & 68 & 68 & 68 & 68 & 68 & 68 & 68 & 68 & 68 & 68\end{array}$

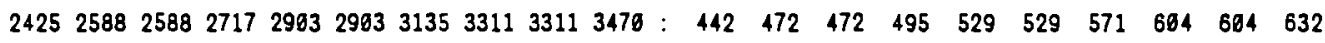
$\begin{array}{lllllllllllllllllllll}2330 & 2493 & 2825 & 2625 & 2812 & 2939 & 2939 & 3118 & 3279 & 3279 & : & 425 & 455 & 479 & 479 & 513 & 536 & 536 & 569 & 598 & 598\end{array}$

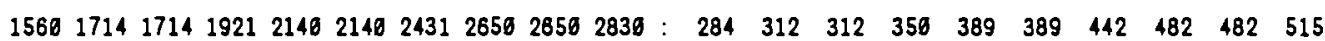

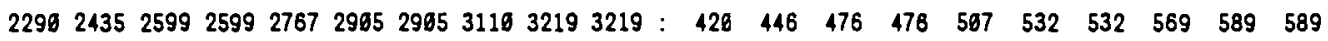
$\begin{array}{lllllllllllllllllllll}1507 & 1559 & 1559 & 1778 & 1778 & 1778 & 1776 & 1776 & 1776 & 1778 & : & 567 & 587 & 587 & 668 & 668 & 668 & 668 & 668 & 668 & 668\end{array}$ $\begin{array}{lllllllllllllllllllll}930 & 974 & 1012 & 1047 & 1047 & 1081 & 1147 & 1193 & 1236 & 1280 & : & 461 & 428 & 436 & 451 & 451 & 465 & 493 & 513 & 531 & 549\end{array}$ $\begin{array}{lllllllllllllllllllll}893 & 937 & 937 & 972 & 1025 & 1958 & 1124 & 1178 & 1217 & 1281 & : & 382 & 462 & 462 & 417 & 439 & 453 & 481 & 501 & 520 & 539\end{array}$

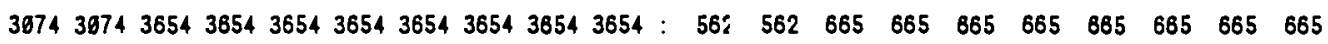
$\begin{array}{lllllllllllllllllllll}1696 & 1141 & 1180 & 1180 & 1238 & 1278 & 1278 & 1329 & 1375 & 1427 & 0 & 437 & 455 & 471 & 471 & 495 & 511 & 511 & 532 & 550 & 572\end{array}$ $\begin{array}{lllllllllllllllllllll}748 & 785 & 836 & 867 & 928 & 963 & 963 & 1021 & 1675 & 1108 & : & 345 & 362 & 383 & 408 & 428 & 444 & 444 & 471 & 496 & 511\end{array}$ $\begin{array}{lllllllllllllllllllll}2895 & 2881 & 3165 & 3165 & 3165 & 3165 & 3165 & 3165 & 3165 & 3165: & 47: & 488 & 571 & 571 & 571 & 571 & 571 & 571 & 571 & 571\end{array}$

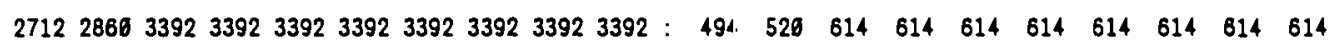

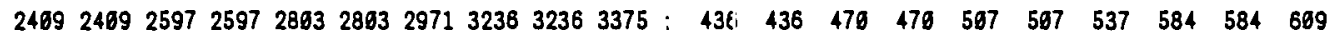
$\begin{array}{lllllllllllllllllllll}946 & 946 & 987 & 1025 & 1025 & 1063 & 1135 & 1135 & 1197 & 1248 & : & 436 & 436 & 455 & 472 & 472 & 490 & 523 & 523 & 552 & 575\end{array}$ $\begin{array}{lllllllllllllllllllll}929 & 978 & 978 & 1016 & 1074 & 1074 & 1146 & 1219 & 1219 & 1275 & : & 4281 & 451 & 451 & 468 & 495 & 495 & 528 & 562 & 562 & 588\end{array}$ $\begin{array}{lllllllllllllllllllll}1113 & 1181 & 1296 & 1286 & 1377 & 1377 & 1377 & 1377 & 1377 & 1377 & : & 51 ; & 539 & 557 & 557 & 639 & 639 & 639 & 639 & 639 & 639\end{array}$ $\begin{array}{lllllllllllllllllllll}1035 & 1635 & 1675 & 1112 & 1112 & 1289 & 1289 & 1289 & 1289 & 1289 & : & 4891 & 480 & 499 & 516 & 518 & 598 & 598 & 598 & 598 & 598\end{array}$ $\begin{array}{lllllllllllllllllllll}1056 & 1656 & 1696 & 1696 & 1154 & 1331 & 1331 & 1331 & 1331 & 1331 & : & 489 & 489 & 588 & 568 & 535 & 817 & 817 & 617 & 817 & 617\end{array}$

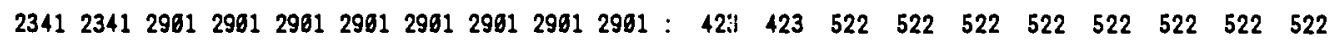
$\begin{array}{lllllllllllllllllllll}1148 & 1148 & 1197 & 1401 & 1401 & 1461 & 1461 & 1401 & 1461 & 1401 & : & 454 & 454 & 473 & 552 & 552 & 552 & 552 & 552 & 552 & 552\end{array}$ $\begin{array}{lllllllllllllllllllll}991 & 1629 & 1673 & 1110 & 1160 & 1197 & 1237 & 1295 & 1350 & 1384 & : & 42 ! & 444 & 463 & 479 & 501 & 517 & 535 & 560 & 584 & 599\end{array}$ $\begin{array}{lllllllllllllllllllll}933 & 971 & 1616 & 1853 & 1162 & 1139 & 1179 & 1237 & 1292 & 1327 & : & 46 ! & 418 & 438 & 454 & 476 & 492 & 569 & 535 & 559 & 574\end{array}$ $\begin{array}{lllllllllllllllllllll}989 & 954 & 1098 & 1837 & 1097 & 1132 & 1176 & 1237 & 1271 & 1339 & : & 39 ! & 411 & 431 & 447 & 474 & 489 & 508 & 535 & 549 & 575\end{array}$

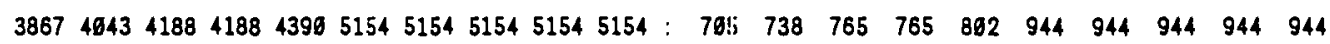
$\begin{array}{lllllllllllllllllllll}3768 & 3768 & 3912 & 4651 & 4651 & 4187 & 4437 & 4437 & 5291 & 5261 & : & 69: 3 & 693 & 719 & 745 & 745 & 769 & 815 & 815 & 955 & 955\end{array}$

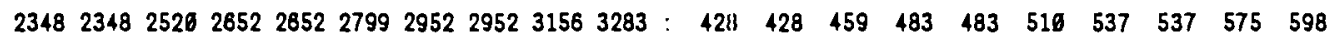

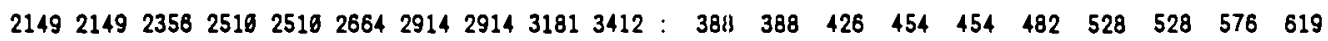

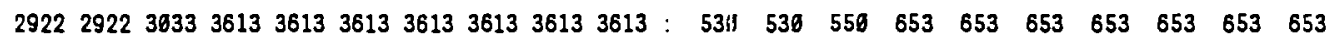
$\begin{array}{lllllllllllllllllllll}919 & 943 & 1064 & 1064 & 1064 & 1064 & 1064 & 1064 & 1064 & 1664 & : & 34 & 356 & 398 & 398 & 398 & 398 & 398 & 398 & 398 & 398\end{array}$ $\begin{array}{lllllllllllllllllllll}899 & 922 & 943 & 1964 & 1064 & 1064 & 1064 & 1064 & 1064 & 1064 & 3311 & 346 & 354 & 397 & 397 & 397 & 397 & 397 & 397 & 397\end{array}$ $\begin{array}{lllllllllllllllllllll}817 & 817 & 841 & 865 & 986 & 986 & 986 & 986 & 986 & 986 & : & 2911 & 296 & 384 & 313 & 357 & 357 & 357 & 357 & 357 & 357\end{array}$ $\begin{array}{lllllllllllllllllllll}866 & 895 & 920 & 920 & 952 & 1073 & 1973 & 1073 & 1073 & 1073 & : & 31 . & 321 & 329 & 329 & 340 & 389 & 380 & 380 & 386 & 380\end{array}$

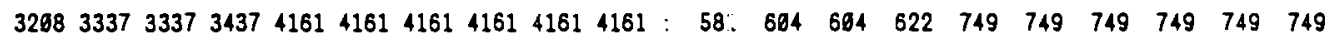

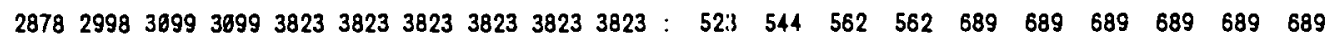
$\begin{array}{lllllllllllllllllllll}862 & 965 & 944 & 975 & 975 & 1012 & 1972 & 1072 & 1118 & 1295 & : & 39 ! & 419 & 437 & 451 & 451 & 468 & 496 & 498 & 517 & 599\end{array}$ $\begin{array}{lllllllllllllllllllll}847 \quad 885 & 1042 & 1042 & 1042 & 1042 & 1042 & 1042 & 1042 & 1042 & : & 36.1 & 381 & 448 & 448 & 448 & 448 & 448 & 448 & 448 & 448\end{array}$ $\begin{array}{lllllllllllllllllllll}1957 & 2642 & 2146 & 2227 & 2347 & 2435 & 2536 & 2668 & 2788 & 2865 & : & 357 & 373 & 392 & 468 & 428 & 444 & 462 & 486 & 569 & 523\end{array}$ $\begin{array}{lllllllllllllllllllll}1934 & 1834 & 1089 & 1126 & 1128 & 1171 & 1247 & 1247 & 1362 & 1356 & : & 47 j & 475 & 496 & 517 & 517 & 538 & 572 & 572 & 598 & 823\end{array}$ $\begin{array}{lllllllllllllllllllll}849 & 905 & 951 & 951 & 1614 & 1660 & 1060 & 1121 & 1176 & 1176 & 39 . & 416 & 437 & 437 & 466 & 487 & 487 & 515 & 540 & 548\end{array}$ $\begin{array}{llllllllllllllllllll}520 & 529 & 529 & 520 & 520 & 520 & 520 & 520 & 520 & 520 & 193 & 193 & 193 & 193 & 193 & 193 & 193 & 193 & 193 & 193\end{array}$ 
TABLE A.4. Middle Case (with Extended Burnup) 1984 Projected Inventories

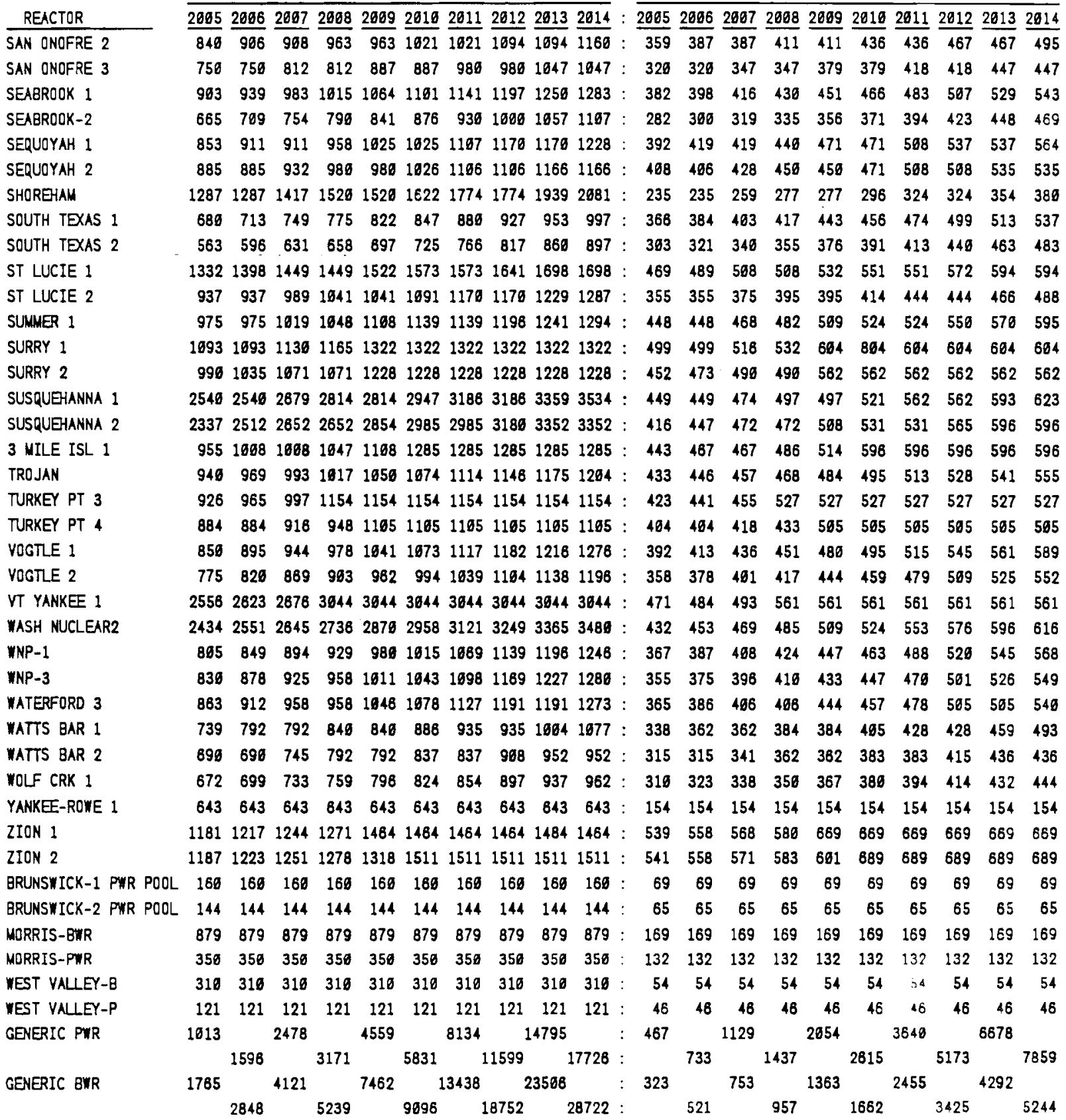


TABLE A.4. Middle Case (with Extended Burnup) 1984 Projected Inventories (contd)

\begin{tabular}{|c|c|c|c|c|c|c|c|c|c|c|c|c|c|}
\hline \multirow[b]{2}{*}{ REACTSR } & \multicolumn{6}{|c|}{ Asseablies } & \multicolumn{7}{|c|}{ MTIHM } \\
\hline & 2815 & 2618 & 2617 & 2618 & 2319 & 2028 & : & 2915 & 2018 & 2917 & 2918 & 2919 & 2028 \\
\hline RX NUCLEAR I & 1339 & 1339 & 1339 & 1339 & 1339 & 1339 & : & 821 & 621 & 621 & 821 & 621 & 621 \\
\hline ARK NUCLEAR 2 & 1371 & 1371 & 1371 & 1371 & 1371 & 1371 & : & 572 & 572 & 572 & 572 & 572 & 572 \\
\hline YAULEY 1 & 489 & 1489 & 1489 & 1489 & 1489 & 1489 & : & 349 & 849 & 849 & 649 & 849 & 849 \\
\hline VAUEY 2 & 914 & 960 & 980 & 996 & 1048 & 1048 & ${ }^{\circ}$ & 421 & 443 & 443 & 459 & 483 & 483 \\
\hline BE_EFONTE 1 & 991 & 1650 & 1958 & 1105 & 1171 & 1171 & : & 450 & 477 & 477 & 582 & 532 & 532 \\
\hline EELIEONTE 2 & 969 & 969 & 1029 & 1694 & 1694 & 1152 & : & 439 & 439 & 468 & 496 & 496 & 523 \\
\hline IG ROCK 1 & 511 & 511 & 511 & 511 & 511 & 511 & : & 68 & 68 & 68 & 66 & 66 & 66 \\
\hline RAICNOOD 1 & 1180 & 1225 & 1281 & 1293 & 1323 & 1376 & : & 499 & 518 & 533 & 547 & 559 & ja2 \\
\hline RAIDHOOD 2 & 1697 & 1141 & 1177 & 1209 & 1239 & 1298 & : & 464 & 482 & 498 & 511 & 524 & 545 \\
\hline RCWNS FERRY1 & 4177 & 4177 & 4177 & 4177 & 4177 & 4177 & $\cdot$ & 766 & 766 & 766 & 766 & 766 & 765 \\
\hline ROWNS FERRY2 & 4988 & 4988 & 4908 & 4988 & 4908 & 4988 & ${ }^{\circ}$ & 899 & 899 & 899 & 899 & 899 & 899 \\
\hline BROWNS FERRY3 & 3899 & 3889 & 4643 & 4807 & 4807 & 4887 & . & 697 & 897 & 739 & 828 & 828 & 828 \\
\hline BRUNSWICK 1 & 3585 & 3585 & 3585 & 3585 & 3585 & 3585 & : & 870 & 870 & 670 & 670 & 878 & 678 \\
\hline BRUNSYICK 2 & 3895 & 3805 & 3895 & 3805 & 3885 & 3805 & : & 712 & 712 & 712 & 712 & 712 & 712 \\
\hline GYRON 1 & 24 & 1289 & 1385 & 1332 & 1383 & 1414 & : & 517 & 536 & 552 & 3 & 576 & 598 \\
\hline YRON 2 & 1172 & 1218 & 1251 & 1283 & 1314 & 1365 & : & 496 & 514 & 529 & 542 & 556 & 577 \\
\hline CALLAMAY 1 & 1184 & 1258 & 1324 & 1324 & 1373 & 1430 & : & 508 & 537 & 565 & 565 & 586 & 610 \\
\hline CALYERT CLF 1 & 1819 & 1819 & 1819 & 1819 & 1819 & 1819 & : & 789 & 709 & 799 & 789 & 709 & 789 \\
\hline CALYERT CLF 2 & 1736 & 1736 & 1736 & 1738 & 1736 & 1738 & : & 615 & 615 & 615 & 815 & 815 & 615 \\
\hline CARROL COUNTY-1 & 593 & 632 & 873 & 714 & 747 & 789 & : & 251 & 287 & 284 & 382 & 316 & 333 \\
\hline CARROL COUNTY-2 & 566 & 605 & 648 & 687 & 729 & 762 & : & 239 & 256 & 273 & 290 & 304 & 322 \\
\hline CATAMBA 1 & 1283 & 1255 & 1294 & 1330 & 1373 & 1411 & : & 510 & 532 & 548 & 563 & 582 & 598 \\
\hline CATAIBA 2 & 1115 & 1151 & 1151 & 1185 & 1218 & 1272 & : & 472 & 488 & 488 & 582 & 516 & 539 \\
\hline CLINTON 1 & 2838 & 2997 & 3109 & 3169 & 3225 & 3355 & : & 519 & 548 & 588 & 68 & 589 & 613 \\
\hline COMANCHE PK 1 & 1367 & 1418 & 1459 & 1495 & 1531 & 1590 & : & 562 & 583 & 599 & 814 & 628 & 652 \\
\hline COMANCHE PK 2 & 1374 & 1425 & 1468 & 1563 & 1540 & 1599 & : & 558 & 578 & 595 & 889 & 824 & 848 \\
\hline $\cos x 1$ & 1774 & 1774 & 1774 & 1774 & 1774 & 1774 & : & 883 & 893 & 863 & 893 & 893 & 893 \\
\hline $\cos 2$ & 1787 & 1787 & 1787 & 1787 & 1787 & 1787 & $:$ & 748 & 740 & 748 & 748 & 740 & 746 \\
\hline COOPER STN & 3144 & 3144 & 3144 & 3144 & 3144 & 3144 & : & 579 & 579 & 579 & 579 & 579 & 579 \\
\hline CRYSTAL RVR 3 & 1969 & 1119 & 1184 & 1341 & 1341 & 1341 & : & 498 & 519 & 540 & $B 22$ & 822 & 622 \\
\hline DAYIS-BESSE 1 & 1156 & 1218 & 1272 & 1449 & 1449 & 1449 & : & 542 & 570 & 596 & 879 & 879 & 679 \\
\hline DIABLO CNYN 1 & 1877 & 1130 & 1181 & 1181 & 1210 & 1258 & : & 495 & 519 & 542 & 542 & 556 & 578 \\
\hline DIABLO CNYN 2 & 1134 & 1134 & 1175 & 1294 & 1254 & 1298 & : & 521 & 521 & 539 & 553 & 576 & 592 \\
\hline DRESDEN 1 & 883 & 683 & 883 & 683 & 683 & 683 & : & 69 & 69 & 69 & 69 & 69 & 69 \\
\hline DRESOEN 2 & 3929 & 3929 & 3929 & 3929 & 3929 & 3929 & : & 718 & 718 & 718 & 718 & 718 & 718 \\
\hline RESOEN 3 & 3761 & 3701 & 3761 & 3781 & 3781 & 3781 & : & 689 & 689 & 689 & 689 & 689 & 689 \\
\hline UANE ARNOLO & 2791 & 2791 & 2791 & 2791 & 2791 & 2791 & : & 512 & 512 & 512 & 512 & 512 & 512 \\
\hline ENRICO FERMI2 & 3653 & 3888 & 4038 & 4038 & 4191 & 4191 & : & 666 & 768 & 736 & 736 & 764 & 764 \\
\hline FARLEY 1 & 1442 & 1442 & 1442 & 1442 & 1442 & 1442 & : & 864 & 664 & 664 & 664 & 664 & 664 \\
\hline FARLEY 2 & 1281 & 1255 & 1412 & 1412 & 1412 & 1412 & : & 554 & 578 & 651 & 851 & 651 & 651 \\
\hline FITZPATRICX & 4103 & 4103 & 4103 & 4103 & 4103 & 4103 & : & 748 & 746 & 746 & 746 & 746 & 746 \\
\hline FORT CALHOUN & 1640 & 1040 & 1040 & 1648 & 1640 & 1046 & : & 376 & 378 & 378 & 378 & 376 & 376 \\
\hline GINNA & 943 & 943 & 943 & 943 & 943 & 943 & : & 344 & 344 & 344 & 344 & 344 & 344 \\
\hline GRAND GUF 1 & 3987 & 4145 & 4145 & 4398 & 4459 & 4632 & : & 694 & 738 & 738 & 784 & 791 & 821 \\
\hline GRAND GUF 2 & 3928 & 4091 & 4258 & 4421 & 4557 & 4728 & : & 722 & 752 & 782 & 813 & 838 & 869 \\
\hline HADOAH NECX & 1387 & 1307 & 1387 & 1397 & $13 \pi 7$ & 1397 & 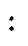 & 538 & 538 & 538 & 538 & 538 & 538 \\
\hline HARRIS 1 & 721 & 762 & 782 & 793 & 829 & 829 & 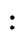 & 335 & 354 & 354 & 369 & 385 & 385 \\
\hline HATCH 1 & 4221 & 4221 & 4221 & 4221 & 4221 & 4221 & & 771 & 771 & 771 & 771 & 771 & 771 \\
\hline
\end{tabular}


TABLE A.4. Middle Case (with Extended Burnup) 1984 Projected Inventories (contd)

\begin{tabular}{|c|c|c|c|c|c|c|c|c|c|c|c|c|c|}
\hline \multirow[b]{2}{*}{ REACTOR } & \multicolumn{6}{|c|}{ Assemblies } & & \multicolumn{6}{|c|}{ MTIHM } \\
\hline & 2015 & 2016 & 2617 & 2618 & 2019 & 2020 & & 2015 & 2616 & 2617 & 2018 & 2819 & 2020 \\
\hline HATCH 2 & 3781 & 3781 & 3781 & 3781 & 3781 & 3781 & & 691 & 691 & 691 & 691 & 691 & 691 \\
\hline HOPE CRK 1 & 3818 & 3986 & 3986 & 4140 & 4329 & 4329 & & 95 & 725 & 725 & 753 & 788 & 788 \\
\hline HUMBOLDT BAY & 396 & 396 & 396 & 396 & 396 & 396 & & 28 & 28 & 28 & 28 & 28 & 28 \\
\hline INDIAN PT 1 & 160 & 166 & 166 & 166 & 166 & 160 & & 31 & 31 & 31 & 31 & 31 & 31 \\
\hline INDIAN PT 2 & 1369 & 1369 & 1369 & 1369 & 1369 & 1369 & & 625 & 625 & 625 & 625 & 625 & 625 \\
\hline INDIAN PT 3 & 385 & 1367 & 1560 & 1568 & 1560 & 1560 & & 595 & 624 & 712 & 712 & 712 & 712 \\
\hline KEMAUNEE & 171 & 1171 & 1171 & 1171 & 1171 & 1171 & & 453 & 453 & 453 & 453 & 453 & 453 \\
\hline LACROSSE & 686 & 666 & 686 & 666 & 606 & 686 & & 68 & 68 & 68 & 68 & 68 & 68 \\
\hline LASALLE CTY 1 & 3587 & 3587 & 3771 & 3915 & 3915 & 4165 & & 654 & 654 & 687 & 714 & 714 & 748 \\
\hline LASALLE CTY 2 & 196 & 3591 & 3591 & 3730 & 3854 & 3854 & & 319 & 655 & 655 & 680 & 763 & 703 \\
\hline LIMERICK 2 & 343 & 3043 & 3250 & 3469 & 3469 & 3668 & & 54 & 554 & 591 & 631 & 31 & 668 \\
\hline LIMERICK 1 & 387 & 3522 & 3522 & 3647 & 3801 & 3801 & & 620 & 644 & 644 & 667 & 695 & 695 \\
\hline MAINE YANKE & 1776 & 1776 & 1776 & 1776 & 1776 & 1776 & & 68 & 668 & 668 & 688 & 68 & 668 \\
\hline MCCUIRE 1 & 1312 & 1368 & 1418 & 1450 & 1494 & 1494 & & 63 & 586 & 668 & 621 & 640 & 646 \\
\hline MCGUIRE 2 & 994 & 1294 & 1344 & 1381 & 1416 & 1458 & & 53 & 553 & 574 & 590 & 85 & 622 \\
\hline MILISTONE 1 & 3654 & 3654 & 3654 & 3654 & 3654 & 3654 & & 65 & 665 & 665 & 665 & 65 & 665 \\
\hline MILLSTONE 2 & 644 & 1644 & 1644 & 1644 & 1644 & 1644 & & 660 & 660 & 660 & 650 & 680 & 668 \\
\hline MILLSTONE 3 & 51 & 1191 & 1229 & 1229 & 1263 & 1320 & & 31 & 550 & 567 & 587 & 83 & 689 \\
\hline MONTICELIO & 65 & 3165 & 3165 & 3165 & 3165 & 3165 & & 71 & 571 & 571 & 571 & 71 & 571 \\
\hline NINE MILE $P$ & 2 & 3 & 3392 & 3392 & 3392 & 3392 & & 14 & 614 & 614 & 14 & 14 & 614 \\
\hline NINE MILE PT2 & 3375 & 3589 & 3589 & 3739 & 3927 & 3927 & & 69 & 848 & 548 & 675 & 68 & 788 \\
\hline NORTH ANNA 1 & 48 & 1311 & 1468 & 1468 & 1468 & 1468 & & 75 & 664 & 676 & 676 & 676 & 676 \\
\hline NORTH ANNA 2 & 1330 & 1330 & 1487 & 1487 & 1487 & 1487 & & 13 & 613 & 586 & 686 & 886 & 686 \\
\hline OCONEE 1 & 77 & 1377 & 1377 & 1377 & 1377 & 1377 & & 639 & 639 & 639 & 639 & 39 & 639 \\
\hline OCONEE 2 & 89 & 1289 & 1289 & 1289 & 1289 & 1289 & & 98 & 598 & 598 & 598 & 98 & 598 \\
\hline OCONEE 3 & 1331 & 1331 & 1331 & 1331 & 1331 & 1331 & & 17 & 617 & 617 & 617 & 17 & 617 \\
\hline OYSTER CRK & 301 & 2901 & 2901 & 2961 & 2901 & 2901 & & 522 & 522 & 522 & 522 & 522 & 522 \\
\hline PALISADES & 1401 & 1461 & 1481 & 1401 & 1481 & 1401 & & 552 & 552 & 552 & 552 & 552 & 552 \\
\hline PALO VERDE 1 & 133 & 1484 & 1525 & 1561 & 1597 & 1655 & & 820 & 843 & 661 & 676 & 692 & 717 \\
\hline PALO VERDE 2 & 1374 & 1424 & 1464 & 1502 & 1538 & 1596 & & 594 & 816 & 634 & 650 & 686 & 691 \\
\hline PALO VERDE 3 & 374 & 1414 & 1448 & 1496 & 1538 & 1575 & & 594 & 612 & 627 & 645 & 65 & 682 \\
\hline PEACHBOTTOM 2 & 5154 & 5154 & 5154 & 5154 & 5154 & 5154 & & 44 & 944 & 944 & 944 & 344 & 944 \\
\hline PEACHBOTTOM 3 & 5201 & 5201 & 5261 & 5261 & 5201 & 5201 & & 55 & 955 & 955 & 955 & 955 & 955 \\
\hline PERRY 1 & 3283 & 3479 & 3634 & 3634 & 3767 & 3984 & & 598 & 633 & 661 & 661 & - 686 & 725 \\
\hline PERRY 2 & 3412 & 3635 & 3861 & 3861 & 4647 & 4282 & & 619 & 659 & 700 & 700 & 134 & 777 \\
\hline PILGRIM 1 & 313 & 3613 & 3613 & 3613 & 3613 & 3613 & & 53 & 853 & 653 & 653 & 3653 & 653 \\
\hline POINT BEACH 1 & 1064 & 1064 & 1064 & 1064 & 1064 & 1064 & & 398 & 398 & 398 & 398 & 398 & 398 \\
\hline POINT BEACH 2 & 1064 & 1864 & 1664 & 1064 & 1664 & 1064 & & 397 & 397 & 397 & 397 & 7 397 & 397 \\
\hline PRAIRIE ISL 1 & 986 & 988 & 986 & 986 & 986 & 986 & & 357 & 357 & 357 & 357 & 357 & 357 \\
\hline PRAIRIE ISL 2 & 1073 & 1073 & 1873 & 1073 & 1073 & 1073 & & 80 & 380 & 380 & 380 & 386 & 380 \\
\hline QUAD CITIES 1 & 4161 & 4181 & 4161 & 4161 & 4161 & 4161 & & 749 & 749 & 749 & 749 & 749 & 749 \\
\hline QUAD CITIES 2 & 3823 & 3823 & 3823 & 3823 & 3823 & 3823 & & 689 & 689 & 689 & 689 & 689 & 689 \\
\hline RANCHO SECO 1 & 1295 & 1295 & 1295 & 1295 & 1295 & 1295 & & 599 & 599 & 599 & 599 & 599 & 599 \\
\hline ROBINSON 2 & 1042 & 1042 & 1042 & 1042 & 1042 & 1042 & & 448 & 448 & 448 & 448 & 448 & 448 \\
\hline RVR BEND 1 & 2977 & 3697 & 3192 & 3280 & 3363 & 3566 & & 543 & 565 & 582 & 598 & 613 & 638 \\
\hline SALEM 1 & 1356 & 1424 & 1488 & 1681 & 1681 & 1681 & & 623 & 654 & 683 & 772 & 2 772 & 772 \\
\hline SALEM 2 & 1219 & 1286 & 1286 & 1338 & 1392 & 1392 & & 560 & 596 & 596 & 813 & 639 & 639 \\
\hline SAN ONOFRE 1 & 520 & 528 & 520 & 520 & 528 & 520 & & 193 & 193 & 193 & 193 & 193 & 193 \\
\hline
\end{tabular}


TABLE A.4. Middle Case (with Extended Burnup) 1984 Projected Inventories (contd)

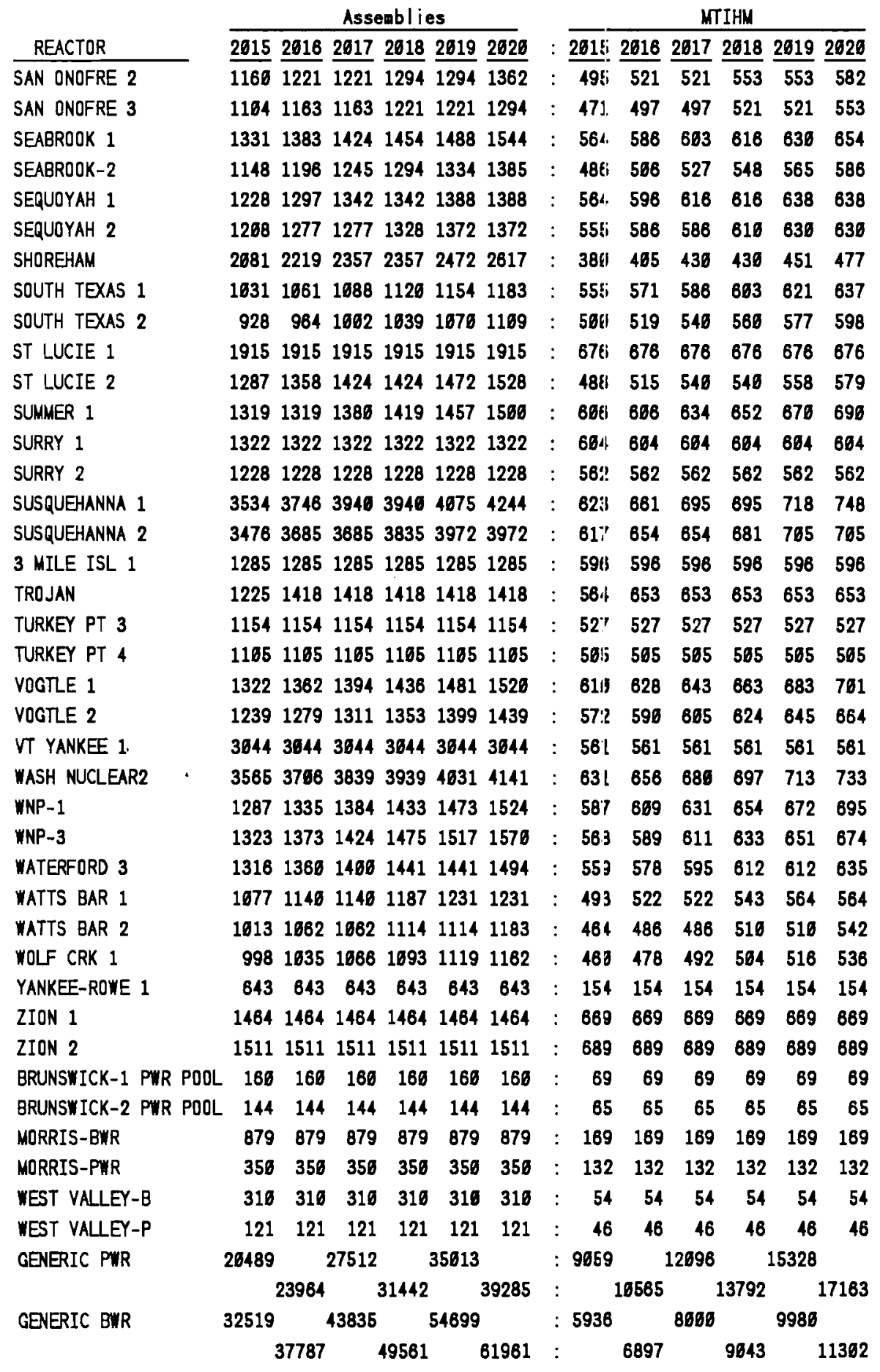


TABLE A.4. Middle Case (with Extended Burnup) 1984 Projected Inventories (contd)

\begin{tabular}{|c|c|c|c|c|c|c|c|c|c|c|c|c|}
\hline & & & & & & BTOTALS & REACTD & YPE AND & TALS & & & \\
\hline & & 1984 & 1985 & 1986 & 1987 & 1988 & 1989 & 1990 & 1991 & 1992 & 1993 & 1994 \\
\hline & & --- & $-\cdots$ & $\cdots$ & --- & --- & --- & $\cdots$ & --- & ---- & --- & ---- \\
\hline PWR & ASSENBLY & 16687 & 17760 & 19788 & 21859 & 24962 & 27337 & 36042 & 33138 & 35575 & 39289 & 41433 \\
\hline PWR & MT. & 6787 & 7590 & 8390 & 9288 & 16587 & 11650 & 12819 & 14140 & 15282 & 16825 & 17743 \\
\hline BWR & ASSEMBLY & 25461 & 27123 & 30727 & 33286 & 37381 & 41236 & 43819 & 48533 & 51412 & 56170 & 59226 \\
\hline BWR & MT. & 4635 & 4936 & 5598 & 6652 & 6796 & 7496 & 7963 & 8816 & 9335 & 16190 & 18746 \\
\hline TOTAL & ASSEMBLY & 41548 & 44883 & 58515 & 55139 & 62283 & 68573 & 73861 & 81671 & 86987 & 95459 & 106653 \\
\hline TOTAL & MT. & 11423 & 12436 & 13979 & 15348 & 17383 & 19146 & 28781 & 22956 & 24537 & 27015 & 28483 \\
\hline
\end{tabular}

\begin{tabular}{|c|c|c|c|c|c|c|c|c|c|c|c|}
\hline & & 1995 & 1996 & 1997 & 1998 & 1999 & 2000 & 2001 & 2002 & 2903 & 2004 \\
\hline & & ---- & ---- & --- & $\cdots$ & $\cdots$ & $\cdots$ & $\cdots$ & $\cdots$ & 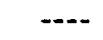 & --- \\
\hline PWR & ASSENBLYY & 45487 & 47749 & 51613 & 54541 & 57174 & 69895 & 63254 & 67310 & 69781 & 73719 \\
\hline PWR & MT. & 19513 & 20497 & 22163 & 23426 & 24574 & 26179 & 27213 & 28982 & 36053 & 31792 \\
\hline BWR & ASSEMBLY & 64281 & 67517 & 71809 & 75925 & 78945 & 83839 & 87120 & 92356 & 95670 & 100969 \\
\hline BWR & MT. & 11656 & 12239 & 13015 & 13757 & 14392 & 15184 & 15776 & 16715 & 17314 & 18269 \\
\hline TDTAL & ASSEMBLY & 109768 & 115266 & 123422 & 138466 & 136119 & 144734 & 156374 & 159666 & 165451 & 174688 \\
\hline TOTAL & MT. & 31189 & 32736 & 35178 & 37184 & 38876 & 41363 & 42989 & 45697 & 47367 & 50961 \\
\hline
\end{tabular}

\begin{tabular}{|c|c|c|c|c|c|c|c|c|c|c|c|}
\hline & & & & & SUB & ALS BY $R$ & CTOR TY & AND TOTA & & & \\
\hline & & 2085 & 2086 & 2087 & 2008 & 2009 & 2010 & 2011 & 2812 & 2013 & 2014 \\
\hline & & --- & --. & --- & ---- & ---- & --- & -- & $--\cdot$ & --- & $-\cdots$ \\
\hline PWR & ASSEMBLY Y & 77074 & 80695 & 84461 & 87968 & 92974 & 97669 & 102446 & 198626 & 114732 & 120651 \\
\hline PWR & MT. & 33265 & 34585 & 36487 & 38066 & 40205 & 42250 & 44393 & 47119 & 49884 & 52327 \\
\hline Bur & ASSEMBLY & 196057 & 116216 & 117987 & 122880 & 130681 & 135575 & 143891 & 151674 & 168174 & 169057 \\
\hline BTR & MT. & 19187 & 19941 & 21342 & 22231 & 23532 & 24533 & 25962 & 27465 & 29012 & 30631 \\
\hline TOTAL & ASSENBLY Y & 183131 & 190311 & 282448 & 210846 & 223855 & 233184 & 245537 & 260380 & 274906 & 289788 \\
\hline TOTAL & $M T$. & 52453 & 54527 & 57830 & 60236 & 63738 & 66784 & 70295 & 74584 & 78817 & 82958 \\
\hline
\end{tabular}


TABLE A.4. Middle Case (with Extended Burnup) 1984 Projected Inventories (contd)

\begin{tabular}{|c|c|c|c|c|c|c|c|}
\hline & & & SUBTO & $S$ BY RE & OR TYPE & D TOTALS & \\
\hline & & 2015 & 2016 & 2617 & 2818 & 2019 & 2620 \\
\hline & & ---- & --- & $\cdots$ & $\cdots$ & ---- & $\cdots$ \\
\hline PWR & ASSEMELY & 125667 & 131579 & 137339 & 143333 & 148368 & 154354 \\
\hline PWR & NT. & 54588 & 57698 & 59618 & 62239 & 64424 & 67608 \\
\hline BWR & ASSEMELY & 177220 & 185232 & 193273 & 201360 & 208592 & 217736 \\
\hline BWR & MT. & 32121 & 33578 & 35046 & 36460 & 37775 & 39436 \\
\hline TOTA & ASSEMBLY & 362887 & 316811 & 336612 & 344693 & 356960 & 372998 \\
\hline TOTA & MT. & 86628 & 96674 & 94656 & 98699 & 182199 & 106444 \\
\hline
\end{tabular}


TABLE A.5. Middle Case (with Extended Burnup) Maximum At-Reactor Capacity-Projected Annual Storage Requirements

ASSENBLIES

NTIHM

POOL 198619871988198919901991199219931994 : 198619871988198919901991199219931994 MILSTONE 2 SURRY 142 MILSTONE 1 PALISADES OCONEE 142 ST LUCIE 1 PEACHBOTTOM 2 PEACHBOTTOM 3 ROBINSON 2 LASALLE CTY $1: 2$ BRUNSWICK 2 PRAIRIE ISL 122 PILGRIM 1 CALVERT CLF 142 LACROSSE OCONEE 3 FITZPATRICK

\begin{tabular}{|c|c|c|c|c|c|c|c|c|c|c|c|c|c|c|c|c|c|}
\hline \\
\hline 38 & 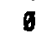 & 57 & 54 & 0 & 59 & 33 & 9 & 46 & 15 & 0 & 23 & 22 & $\theta$ & 24 & 13 & 6 & 19 \\
\hline \multirow[t]{17}{*}{15} & б & 44 & 85 & 44 & 59 & 65 & 57 & 37 & 7 & 0 & 28 & 39 & 28 & 27 & 30 & 28 & 17 \\
\hline & 26 & ฮ & 124 & $\theta$ & 182 & $\theta$ & 195 & 112 & : & 5 & $g$ & 22 & D & 32 & 0 & 34 & 26 \\
\hline & & 37 & 0 & 6 & 67 & 33 & פ & 40 & : & & 14 & פ & 0 & 28 & 13 & 6 & 16 \\
\hline & & 1 & 44 & 45 & 57 & 38 & 136 & 39 & : & & ๑ & 20 & 21 & 28 & 18 & 60 & 18 \\
\hline & & $B 1$ & $\theta$ & 55 & 89 & 6 & 77 & 62 & : & & 18 & 0 & 28 & 27 & 6 & 29 & 19 \\
\hline & & 65 & 167 & $\sigma$ & 228 & 132 & 6 & 148 & : & & 12 & 30 & 0 & 42 & 24 & 6 & 27 \\
\hline & & & & 127 & ס & 132 & 239 & 0 & : & & & & 23 & $\theta$ & 24 & 44 & $\theta$ \\
\hline & & & & & 9 & 29 & 49 & 0 & : & & & & & 4 & 12 & 21 & 6 \\
\hline & & & & & 319 & 128 & 213 & 277 & : & & & & & 58 & 23 & 39 & 50 \\
\hline & & & & & & 62 & 171 & 0 & : & & & & & & 12 & 32 & 6 \\
\hline & & & & & & 3 & 38 & 24 & : & & & & & & 1 & 13 & 9 \\
\hline & & & & & & & 148 & D & : & & & & & & & 26 & $\theta$ \\
\hline & & & & & & & & 84 & : & & & & & & & & 33 \\
\hline & & & & & & & & $B$ & & & & & & & & & 1 \\
\hline & & & & & & & & 24 & : & & & & & & & & 11 \\
\hline & & & & & & & & 49 & : & & & & & & & & 9 \\
\hline & & & & & & & & & : & & & & & & & & \\
\hline \multirow[t]{2}{*}{53} & & 260 & 183 & 144 & 346 & 201 & 351 & 358 & 22 & & 77 & 81 & $B 1$ & 134 & 87 & 149 & 141 \\
\hline & 28 & 65 & 291 & 127 & 727 & 454 & 986 & 590 & : & 5 & 12 & 53 & 23 & 132 & 83 & 175 & 187 \\
\hline 53 & $2 B$ & 265 & 474 & 271 & 1067 & 655 & 1317 & 946 & 22 & 5 & 89 & 134 & 85 & $28 B$ & 171 & 324 & 248 \\
\hline
\end{tabular}


TABLE A.5. Middle Case (with Extended Burnup) Maximum At-Reactor Capacity-Projected Annual Storage Requirements (contd)

ASSENBLIES

MTIHN

\begin{tabular}{|c|}
\hline POOL \\
\hline MILISTONE 2 \\
\hline SURRY $1 k 2$ \\
\hline MILLSTONE 1 \\
\hline PALISADES \\
\hline OCONEE 182 \\
\hline ST LUCIE 1 \\
\hline PEACHBOTTOM 2 \\
\hline PEACHBOTTOH 3 \\
\hline ROBINSON 2 \\
\hline LASALLE CTY 112 \\
\hline BRUNSIICK 2 \\
\hline PRAIRIE ISL 112 \\
\hline PILGRIU 1 \\
\hline CALVERT QLF 112 \\
\hline LACROSSE \\
\hline DCONEE 3 \\
\hline FITZPATRICK \\
\hline BRUNSWICK 1 \\
\hline INDIAN PT 3 \\
\hline BYRON $1: 2$ \\
\hline COOPER STN \\
\hline FORT CALHOUN \\
\hline DAVIS-BESSE 1 \\
\hline BIG ROCK 1 \\
\hline POINT BEACH $1 \& 2$ \\
\hline ARK NUCLEAR 1 \\
\hline BRAIDYOOD 112 \\
\hline INDIAN PT 2 \\
\hline ENRICO FERMI2 \\
\hline B VALLEY 1 \\
\hline OYSTER CRK 1 \\
\hline SEQUOYAH $1 \mathbf{2}$ \\
\hline ZION $1 \& 2$ \\
\hline MAINE YANKEE \\
\hline BROWNS FERRY2 \\
\hline COOK 112 \\
\hline MILLSTONE 3 \\
\hline SAN ONOFRE $1,2, L 3$ \\
\hline WASH MUCLEAR2 \\
\hline ARK NUCLEAR 2 \\
\hline PERRY 112 \\
\hline
\end{tabular}

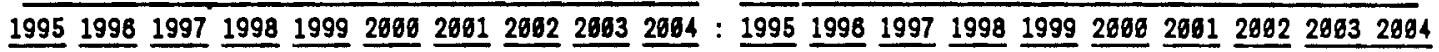

\begin{tabular}{|c|c|c|c|c|c|c|c|c|c|c|c|c|c|c|c|c|c|c|c|c|}
\hline 51 & 6 & 67 & 49 & 6 & 58 & 35 & 6 & 38 & 61 & : & 21 & 6 & 27 & 20 & 9 & 24 & $\sqrt{14}$ & 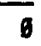 & 15 & \\
\hline 102 & 35 & 64 & 72 & 41 & 57 & 71 & 83 & 36 & 94 & : & 47 & 16 & 29 & 33 & 19 & 26 & 33 & 29 & 17 & \\
\hline 0 & 110 & 6 & 121 & 6 & 188 & $\theta$ & 288 & 167 & 6 & & 9 & 28 & 0 & 21 & $\theta$ & 36 & $\theta$ & 37 & 19 & \\
\hline 84 & 34 & 6 & 41 & 45 & 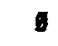 & 34 & 66 & 6 & 53 & : & 25 & 13 & 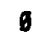 & 16 & 18 & $\theta$ & 13 & 26 & $\theta$ & \\
\hline 57 & 77 & 68 & 46 & 45 & 66 & 38 & 132 & 38 & 52 & : & 26 & 36 & 32 & 19 & 21 & 31 & 18 & 61 & 18 & \\
\hline 69 & $\theta$ & 96 & 52 & 0 & 81 & 52 & ø & 84 & 63 & : & 26 & 0 & 30 & 19 & 0 & 25 & 19 & $\theta$ & 28 & \\
\hline 280 & g & 247 & 144 & 6 & 220 & 143 & g & 145 & 188 & ： & 37 & g & 46 & 27 & 6 & 41 & 26 & ฤ & 27 & \\
\hline 298 & 143 & b & 148 & 175 & פ & 142 & 258 & 6 & 196 & : & 38 & 26 & 8 & 27 & 32 & 6 & 26 & 47 & 8 & \\
\hline 42 & 36 & 54 & 31 & ø & 48 & 31 & 53 & 30 & b & : & 18 & 13 & 23 & 13 & 6 & 21 & 13 & 23 & 13 & \\
\hline 190 & 126 & 460 & 133 & 156 & 415 & 132 & 230 & 258 & 176 & : & 34 & 23 & 85 & 24 & 28 & 76 & 24 & 42 & 47 & \\
\hline 148 & 101 & 6 & 108 & 127 & 8 & 93 & 183 & 6 & 141 & : & 28 & 19 & (1) & 20 & 24 & $\theta$ & 17 & 34 & $\theta$ & \\
\hline 85 & 50 & 82 & 50 & 28 & 75 & 25 & 82 & 50 & 62 & : & 23 & 17 & 29 & 17 & 9 & 28 & 9 & 29 & 17 & \\
\hline 157 & 168 & 6 & 115 & b & 180 & 0 & 195 & 8 & 154 & : & 28 & 19 & 8 & 20 & 6 & 32 & 6 & 35 & 0 & \\
\hline 58 & 48 & 148 & 48 & 53 & 134 & 50 & 76 & 98 & 59 & : & 23 & 19 & 58 & 19 & 21 & 53 & 26 & 30 & 38 & \\
\hline 20 & 13 & 25 & 14 & 17 & 23 & 11 & 24 & 13 & 6 & & 2 & 1 & 3 & 2 & 2 & 3 & 1 & 3 & 1 & \\
\hline 58 & 6 & 72 & 6 & 47 & 6 & 38 & 72 & 0 & 54 & : & 26 & b & 33 & 0 & 22 & 6 & 18 & 33 & ซ & \\
\hline 143 & 6 & 175 & 107 & 0 & 160 & 168 & 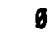 & 196 & 137 & : & 26 & 0 & 31 & 19 & 6 & 29 & 19 & (1) & 19 & \\
\hline 103 & 6 & 184 & 107 & ø & 186 & 93 & g & 98 & 141 & : & 19 & 0 & 34 & 28 & $\theta$ & 31 & 17 & $g$ & 18 & \\
\hline \multirow[t]{23}{*}{2} & 6 & 79 & 55 & פ & 68 & 0 & 89 & 43 & $\emptyset$ & : & 1 & $\theta$ & 38 & 25 & 0 & 31 & 6 & 41 & 28 & \\
\hline & 26 & 96 & 89 & 70 & 97 & 59 & 168 & 60 & 88 & : & & 11 & 41 & 38 & 30 & 41 & 25 & 46 & 25 & \\
\hline & 64 & 118 & 69 & 89 & 165 & 68 & 119 & 68 & 87 & : & & 12 & 22 & 13 & 15 & 19 & 12 & 22 & 12 & \\
\hline & 12 & 41 & g & 39 & 45 & 0 & 41 & 25 & ø & : & & 4 & 15 & 6 & 14 & 16 & 6 & 15 & 9 & \\
\hline & 25 & b & 40 & 48 & 0 & 37 & 71 & $\theta$ & 53 & : & & 12 & g & 19 & 22 & 6 & 17 & 33 & б & \\
\hline & & 6 & 14 & 17 & 18 & 15 & ø & 0 & 0 & : & & & 1 & 2 & 2 & 2 & 2 & g & 8 & \\
\hline & & 39 & 44 & 45 & 58 & 44 & 89 & 44 & 52 & : & & & 14 & 16 & 16 & 21 & 16 & 24 & 16 & \\
\hline & & & 40 & 0 & 63 & 0 & 71 & ø & 54 & 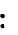 & & & & 19 & 0 & 29 & 6 & 33 & 6 & \\
\hline & & & 80 & 71 & 97 & 80 & 188 & 80 & 87 & : & & & & 34 & 30 & 41 & 25 & 46 & 25 & \\
\hline & & & 35 & 51 & g & 41 & 74 & 6 & 57 & 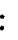 & & & & 18 & 23 & 6 & 19 & 34 & 6 & \\
\hline & & & 224 & 8 & 249 & $\theta$ & 271 & 158 & 6 & 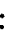 & & & & 41 & 0 & 45 & 6 & 49 & 29 & \\
\hline & & & 4 & 63 & 0 & 37 & 78 & g & 59 & : & & & & 2 & 24 & $\theta$ & 17 & 36 & 8 & \\
\hline & & & 41 & 6 & 158 & 186 & 6 & 98 & 6 & : & & & & 7 & g & 28 & 19 & $\theta$ & 17 & \\
\hline & & & 1 & 67 & 147 & 48 & 81 & 97 & 62 & : & & & & & 26 & 67 & 22 & 37 & 45 & \\
\hline & & & & 39 & 90 & 56 & 192 & 54 & 78 & : & & & & & 18 & 41 & 26 & 47 & 25 & \\
\hline & & & & 19 & 88 & 6 & 74 & 43 & 6 & : & & & & & 7 & 25 & 6 & 28 & 16 & \\
\hline & & & & 81 & g & 162 & 6 & 158 & 6 & 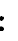 & & & & & 15 & 0 & 29 & 6 & 29 & \\
\hline & & & & & 91 & 48 & 91 & 194 & 65 & 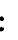 & & & & & & 37 & 22 & 37 & 45 & \\
\hline & & & & & 14 & 37 & ø & 39 & 43 & : & & & & & & 8 & 17 & 6 & 18 & \\
\hline & & & & & 147 & 116 & ઇ & 118 & 70 & & & & & & & 55 & 50 & 6 & 50 & \\
\hline & & & & & & 51 & 165 & 91 & 128 & & & & & & & & 9 & 29 & 16 & \\
\hline & & & & & & & 34 & g & 56 & & & & & & & & & 14 & g & \\
\hline & & & & & & & 372 & 6 & 438 & & & & & & & & & 68 & $\theta$ & \\
\hline
\end{tabular}


TABLE A.5. Middle Case (with Extended Burnup) Maximum At-Reactor Capacity-Projected Annual Storage Requirements (contd)

ASSEMBLIES

NTIHM

POOL ORESDEN 2 HADDAN NECK

DUANE ARNOLD NINE MILE PT1 BROWNS FERRY1 VATTS BAR 112 VT YANKEE 1 NORTH ANMA $1: 2$ KEIAUNEE ST LUCIE 2 LIMERICK 1 SALEU 1

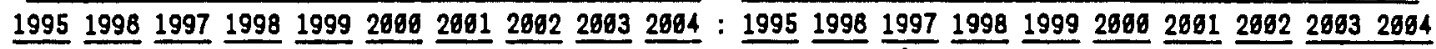

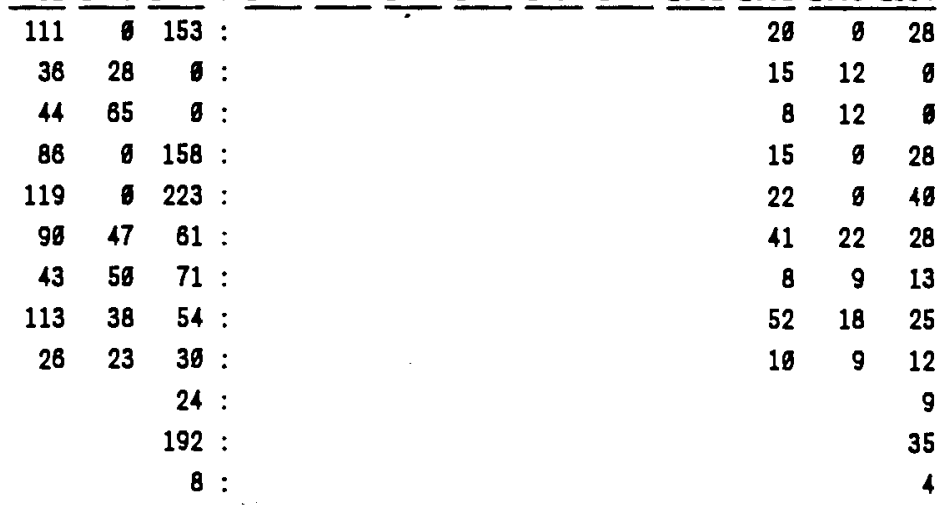

PIR TOTAL BNR TOTAL

TOTAL

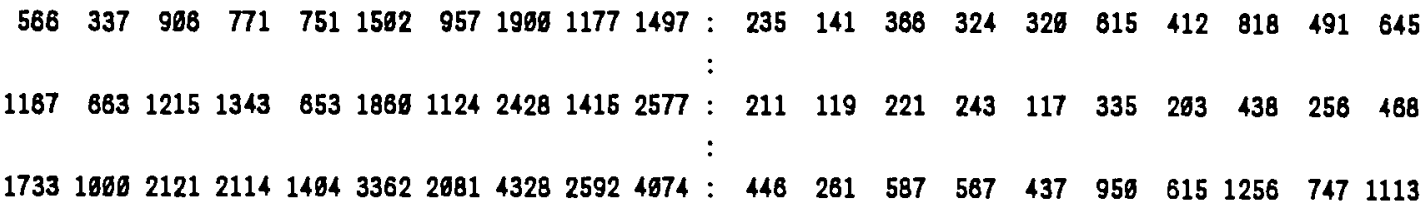


TABLE A.5. Middle Case (with Extended Burnup) Maximum At-Reactor Capacity-Projected Annual Storage Requirements (contd)

ASSEMBLIES

WTIHM

POOL

MIUSTONE 2

SURRY 112

MIUSTONE 1

PALISADES

OCONEE 122

ST LUCIE 1

PEACHBOTTON 2

PEACHBOTTON 3

ROBINSON 2

LASNLE CTY 112

BRUNSWICK 2

PRAIRIE ISL 112

PILGRIM 1

CALVERT CLF 122

LACROSSE

OCONEE 3

FITZPATRICK

BRUNSWICK 1

INDIAN PT 3

BYRON 112

FORT CALHOUN

DAVIS-BESSE 1

POINT BEACH 112

ARK NUCLEAR 1

BRAIDWOOD 112

INDIAN PT 2

ENRICO FERMI2

B VNLLEY 1

OYSTER CRK 1

SEQUOYAH $1 \mathbf{2}$

ZION $1 \mathbf{2}$

MAINE YANKEE

BROWNS FERRY2

COOK 112

MILLSTONE 3

SAN ONOFRE $1,2,23$

MASH NUCLEAR2

ARK NUCLEAR 2

PERRY 112

DRESDEN 2

HADDAM NECX

DUANE ARNOLD

NIME MILE PT1

BROWNS FERRY1

IATTS QAR 122

\begin{tabular}{|c|c|c|c|c|c|c|c|c|c|c|c|c|c|c|c|c|c|c|c|}
\hline 685 & 2006 & 2067 & $\underline{2968}$ & 2009 & $\underline{2010}$ & 2011 & 2912 & $\underline{2013}$ & 2014 & $: 2665$ & 2666 & $\underline{2607}$ & 2668 & $\underline{2089}$ & $\underline{2010}$ & $\underline{2011}$ & $\underline{2012}$ & $\underline{2013}$ & 2014 \\
\hline 6 & 45 & 39 & 6 & 58 & 48 & 6 & 51 & 46 & 52 & 8 & 18 & 16 & 6 & 24 & 16 & 8 & 21 & 19 & 21 \\
\hline 38 & 45 & 73 & 35 & 157 & 6 & 6 & 6 & 0 & 0 & 17 & 21 & 34 & 16 & 72 & 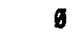 & 0 & 6 & ( & g \\
\hline 117 & 6 & 0 & 6 & 6 & 0 & 6 & 6 & 6 & 6 & 21 & $\theta$ & 0 & 6 & 6 & 0 & פ & 6 & 0 & ø \\
\hline 48 & g & 49 & 6 & 0 & $\theta$ & 0 & 0 & 0 & 0 & 16 & $\theta$ & 19 & 6 & 0 & 0 & 0 & 0 & 8 & $\theta$ \\
\hline 46 & 48 & 79 & 37 & 177 & $\theta$ & 6 & 6 & $\theta$ & 0 & 21 & 22 & 37 & 17 & 82 & 8 & 6 & 6 & $\theta$ & $\theta$ \\
\hline 0 & 86 & 51 & 0 & 73 & 51 & 6 & 68 & 57 & 0 & ( & 21 & 19 & $\theta$ & 23 & 19 & D & 22 & 21 & 0 \\
\hline 6 & 176 & 145 & D & 262 & 0 & $\theta$ & 0 & 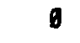 & 0 & 0 & 33 & 27 & 6 & 37 & g & 0 & 6 & 6 & $g$ \\
\hline 164 & $\theta$ & 144 & 139 & 6 & 136 & 250 & $\theta$ & 0 & 6 & 30 & 0 & 26 & 25 & 0 & 25 & 46 & 6 & 6 & $\theta$ \\
\hline 33 & 38 & 6 & 0 & 6 & $\theta$ & $\theta$ & 6 & 0 & 0 & 14 & 16 & 6 & $\theta$ & $\theta$ & 0 & D & 6 & 0 & 0 \\
\hline 146 & 328 & 132 & 129 & 373 & 127 & 232 & 355 & 161 & 159 & 27 & 59 & 24 & 23 & 68 & 23 & 42 & 65 & 29 & 29 \\
\hline 117 & 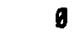 & 104 & 91 & 6 & 89 & 177 & 6 & 129 & 127 & 22 & 0 & 19 & 17 & 0 & 17 & 33 & g & 24 & 24 \\
\hline 53 & 29 & 49 & 24 & 153 & 0 & 6 & 0 & 0 & $\theta$ & 19 & 10 & 17 & 9 & 55 & 0 & 0 & 0 & 0 & $\theta$ \\
\hline 127 & $\theta$ & 111 & 0 & 6 & 0 & 0 & 0 & 0 & 0 & 22 & 0 & 20 & $\theta$ & 0 & 0 & 6 & 6 & 0 & $\theta$ \\
\hline 48 & 107 & 48 & 48 & 121 & 48 & 76 & 102 & 54 & 217 & 19 & 42 & 19 & 19 & 48 & 19 & 36 & 48 & 21 & 54 \\
\hline פ & $\theta$ & 0 & D & 0 & 0 & 0 & 0 & 0 & 0 & 0 & 0 & 0 & 0 & 0 & 0 & 0 & g & $\theta$ & 0 \\
\hline 45 & 0 & 40 & & 58 & 0 & 0 & $\theta$ & 0 & 0 & 21 & D & 19 & 0 & 27 & 0 & 0 & D & 0 & 0 \\
\hline 0 & 128 & 165 & 0 & 145 & 184 & 6 & 142 & 127 & 125 & D & 23 & 19 & $\theta$ & 26 & 19 & 6 & 26 & 23 & 22 \\
\hline 6 & 132 & 105 & $b$ & 152 & 89 & 6 & 145 & 135 & 132 & 0 & 25 & 20 & b & 28 & 17 & 0 & 27 & 25 & 25 \\
\hline 55 & g & 55 & 50 & 0 & 49 & g & 59 & 54 & 0 & 25 & 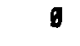 & 25 & 23 & 0 & 22 & 6 & 27 & 25 & ø \\
\hline 88 & 84 & 80 & 58 & 85 & 64 & 71 & 101 & 94 & 56 & 37 & 27 & 34 & 25 & 36 & 27 & 30 & 43 & 40 & 24 \\
\hline 38 & 48 & $\theta$ & 30 & 0 & 0 & 0 & 0 & 0 & 0 & 14 & 14 & - & 11 & 0 & 0 & 0 & 6 & 6 & 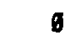 \\
\hline 44 & 6 & 39 & 37 & 0 & 36 & 69 & 6 & 50 & 49 & 21 & 0 & 18 & 17 & 6 & 17 & 32 & 6 & 23 & 23 \\
\hline 42 & 47 & 142 & 0 & 6 & g & b & $\theta$ & 6 & g & 15 & 17 & 59 & 0 & $\theta$ & 0 & - & 6 & 6 & 0 \\
\hline 45 & 0 & 41 & 0 & 57 & 0 & 0 & 0 & 0 & 6 & 21 & 0 & 19 & $\theta$ & 28 & 0 & 0 & 0 & 6 & 0 \\
\hline 89 & 63 & 80 & 80 & 86 & 64 & 73 & 161 & 98 & 80 & 38 & 27 & 34 & 25 & 36 & 27 & 31 & 43 & 41 & 25 \\
\hline 48 & 0 & 42 & 41 & 0 & - & 0 & 6 & 0 & 6 & 22 & 0 & 19 & 19 & 0 & 0 & 0 & 0 & 0 & ๑ \\
\hline 224 & 0 & 204 & D & 224 & 147 & 6 & 259 & 0 & 152 & 41 & 0 & 37 & 0 & 41 & 27 & 0 & 47 & 6 & 28 \\
\hline 48 & 0 & 43 & 38 & 0 & 36 & 75 & 6 & 53 & 52 & 22 & g & 29 & 17 & 0 & 17 & 35 & 0 & 24 & 24 \\
\hline 124 & 8 & 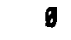 & 6 & 0 & 0 & 0 & 0 & 0 & 0 & 22 & 0 & 0 & 1 & 0 & 0 & 6 & 6 & 6 & $\theta$ \\
\hline 52 & 58 & 47 & 95 & 67 & 46 & 162 & 63 & 60 & 58 & 24 & 27 & 22 & 44 & 31 & 21 & 74 & 29 & 28 & 27 \\
\hline 85 & 72 & 55 & 54 & 233 & $\theta$ & $\theta$ & $\theta$ & g & 6 & 30 & 33 & 25 & 25 & 107 & 0 & 0 & D & $\theta$ & $\theta$ \\
\hline 46 & 52 & 0 & 0 & 0 & 0 & 0 & 0 & 0 & 0 & 17 & 20 & 0 & 0 & 0 & 0 & 0 & 0 & 0 & 0 \\
\hline 184 & $\theta$ & 165 & 0 & 236 & 0 & $\theta$ & 235 & 0 & 216 & 33 & 6 & 30 & $\theta$ & 43 & $\theta$ & 6 & 43 & $\theta$ & 39 \\
\hline 57 & 60 & 187 & 55 & 87 & 56 & 170 & 61 & 86 & 193 & 23 & 28 & 46 & 22 & 31 & 23 & 73 & 28 & 27 & 83 \\
\hline 52 & 37 & 45 & 37 & 61 & 35 & 8 & 58 & 54 & 33 & 24 & 17 & 21 & 17 & 28 & 16 & 6 & 27 & 25 & 15 \\
\hline 80 & 66 & 62 & 57 & 75 & 58 & 93 & 73 & $B 7$ & 86 & 26 & 28 & 26 & 24 & 32 & 25 & 46 & 31 & 29 & 28 \\
\hline 105 & 117 & 94 & 91 & 134 & 88 & 163 & 128 & 118 & 115 & 18 & 21 & 17 & 16 & 24 & 15 & 29 & 23 & 20 & 28 \\
\hline 49 & 0 & 49 & 0 & 80 & 0 & 78 & 59 & 0 & 9 & 20 & ( & 20 & 0 & 25 & D & 32 & 25 & 0 & 6 \\
\hline 415 & 0 & 379 & 286 & - & 361 & 463 & $\theta$ & 471 & 358 & 72 & 6 & 69 & 52 & 0 & 55 & 73 & 0 & 88 & 65 \\
\hline 128 & 6 & $\theta$ & ฮ & 0 & 0 & 6 & $\theta$ & $\theta$ & 0 & 23 & 6 & 0 & 0 & 0 & D & 6 & 0 & $\theta$ & b \\
\hline 35 & 46 & 8 & 1 & 8 & 6 & $\theta$ & 8 & $\theta$ & $\theta$ & 14 & 16 & 6 & $\theta$ & 0 & 6 & 0 & 0 & $\theta$ & 6 \\
\hline 77 & 87 & $\theta$ & 85 & 99 & 6 & 124 & 94 & 1 & 86 & 14 & 16 & 0 & 12 & 18 & 6 & 23 & 17 & 6 & 16 \\
\hline - & 148 & $\theta$ & 6 & D & 0 & 6 & ○ & 0 & 6 & g & 26 & g & $\theta$ & 0 & 0 & $\theta$ & 0 & 1) & 6 \\
\hline 6 & 206 & 0 & 158 & 6 & 6 & 0 & - & $\theta$ & $\theta$ & 0 & 37 & 0 & 29 & $g$ & .0 & 0 & g & 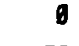 & $\theta$ \\
\hline 112 & 53 & 56 & 95 & 6 & 91 & 49 & 71 & 113 & 73 & 51 & 24 & 25 & 44 & 6 & 42 & 22 & 33 & 52 & 34 \\
\hline
\end{tabular}


TABLE A.5. Middle Case (with Extended Burnup) Maximum At-Reactor Capacity-Projected Annual Storage Requirements (contd)

ASSEMRIES

MTIHM

POOL

$\checkmark T$ YANKEE 1

\begin{tabular}{|c|c|c|c|c|c|c|c|c|c|c|c|c|c|c|c|c|c|c|c|}
\hline 2065 & 2068 & 2097 & 2068 & 2699 & 2010 & 2011 & 2612 & 22013 & 2014 & $: 2605$ & 2668 & 2687 & 2608 & 2669 & 2016 & 2011 & 2012 & 2613 & 2614 \\
\hline 88 & 67 & 53 & 8 & 6 & $\theta$ & 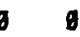 & $\emptyset$ & 6 & 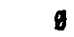 & 11 & $\overline{12}$ & 10 & 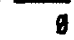 & $\bar{\theta}$ & $\overline{6}$ & 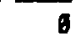 & $\vec{\theta}$ & $\overline{8}$ & 8 \\
\hline 96 & 49 & 41 & 76 & 58 & 38 & $8 \quad 144$ & 73 & 62 & 187 & 42 & 23 & 19 & 35 & 27 & 18 & 68 & 34 & 29 & 48 \\
\hline 25 & 28 & 22 & 23 & 31 & 0 & $g$ & $\theta$ & 8 & $\theta$ & 10 & 11 & 8 & 9 & 12 & $\theta$ & $\theta$ & 9 & $\theta$ & 6 \\
\hline 54 & 0 & 52 & 52 & $\theta$ & 58 & 79 & 6 & 59 & 58 & 20 & $\theta$ & 26 & 26 & 6 & 19 & 30 & 0 & 22 & 22 \\
\hline 6 & 145 & 164 & 0 & 188 & 138 & $\theta$ & 205 & $5 \quad 189$ & $\theta$ & $\theta$ & 28 & 30 & $\theta$ & 31 & 25 & $\theta$ & 37 & 20 & g \\
\hline 49 & 6 & 46 & 48 & 6 & 45 & 76 & 6 & 55 & 54 & 22 & 8 & 21 & 21 & 6 & 21 & 35 & 8 & 25 & 25 \\
\hline \multirow[t]{32}{*}{80} & 6 & 164 & ต & 238 & g & g 288 & 6 & 207 & 6 & 11 & 0 & 30 & 6 & 43 & $\varnothing$ & 52 & 6 & 38 & 6 \\
\hline & 71 & 93 & 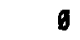 & g & ø & g & g & g & 6 & : & 13 & 17 & 6 & 6 & 6 & D & $\theta$ & ต & ต \\
\hline & 127 & 153 & 130 & 217 & 130 & d 282 & $2 \quad 294$ & $4 \quad 193$ & 566 & : & 23 & 28 & 24 & 46 & 24 & 48 & 37 & 35 & 162 \\
\hline & 38 & 98 & 88 & 122 & B4 & 89 & 130 & 88 & 118 & : & 17 & 46 & 31 & 56 & 30 & 41 & 60 & 31 & 54 \\
\hline & 136 & 279 & 135 & 202 & 284 & $4 \quad 239$ & 195 & $5 \quad 345$ & 175 & : & 24 & 49 & 24 & 36 & 46 & 42 & 34 & 61 & 31 \\
\hline & 9 & 6 & $\theta$ & 0 & 0 & 9 & 0 & 8 & 6 & : & 3 & 6 & 6 & 6 & $\emptyset$ & 0 & 0 & 0 & 6 \\
\hline & 2 & 39 & 31 & g & 37 & 80 & g & 46 & 6 & : & 1 & 18 & 14 & g & 17 & 28 & g & 21 & 0 \\
\hline & & 30 & 189 & g & o & g & g & 0 & g & : & & 14 & 87 & 6 & 0 & 0 & 0 & 0 & 6 \\
\hline & & 37 & 116 & 0 & 119 & $9 \quad 144$ & g & g 178 & 198 & : & & 7 & 21 & 6 & 22 & 28 & 0 & 33 & 36 \\
\hline & & 21 & 35 & 51 & 35 & 54 & 70 & 57 & 50 & : & & 10 & 16 & 23 & 16 & 25 & 32 & $2 B$ & 23 \\
\hline & & 46 & 33 & 53 & 32 & 55 & 71 & 58 & 53 & : & & 26 & 14 & 23 & 14 & 24 & 31 & 25 & 23 \\
\hline & & & 22 & 50 & 37 & 40 & 58 & 55 & 34 & : & & & 16 & 22 & 16 & 17 & 25 & 24 & 15 \\
\hline & & & & 14 & 37 & 40 & 58 & 55 & 35 & : & & & & 6 & 16 & 17 & 25 & 24 & 15 \\
\hline & & & & 9 & 35 & 44 & 61 & 34 & 59 & : & & & & 4 & 15 & 19 & 27 & 15 & 28 \\
\hline & & & & 430 & ต & 0 & 0 & 6 & 0 & : & & & & 76 & 6 & ต & $\theta$ & g & g \\
\hline & & & & 42 & ต & g 291 & 219 & 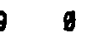 & 189 & : & & & & 8 & 6 & 53 & 46 & 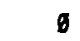 & 33 \\
\hline & & & & 21 & 37 & 46 & 58 & 53 & 33 & : & & & & 9 & 16 & 17 & 24 & 22 & 14 \\
\hline & & & & 37 & 46 & 6 & 61 & 55 & $\theta$ & : & & & & 17 & 21 & 6 & 28 & 25 & 6 \\
\hline & & & & & 26 & 6 & 57 & 45 & 53 & : & & & & & 9 & 0 & 26 & 21 & 24 \\
\hline & & & & & & 9 & 0 & 40 & 40 & : & & & & & & 4 & 6 & 19 & 19 \\
\hline & & & & & & & 35 & 6 & 6 & : & & & & & & & 16 & 6 & 6 \\
\hline & & & & & & & 118 & 122 & 77 & : & & & & & & & 22 & 22 & 14 \\
\hline & & & & & & & 42 & 6 & 82 & : & & & & & & & 18 & 6 & 35 \\
\hline & & & & & & & 184 & 141 & g & : & & & & & & & 33 & 26 & 6 \\
\hline & & & & & & & 16 & 49 & 0 & : & & & & & & & 7 & 22 & 6 \\
\hline & & & & & & & & 30 & 50 & : & & & & & & & & 13 & 21 \\
\hline & & & & & & & & 49 & 0 & : & & & & & & & & 22 & 6 \\
\hline & & & & & & & & 38 & 71 & : & & & & & & & & 15 & 29 \\
\hline & & & & & & & & & 10 & : & & & & & & & & & 4 \\
\hline & & & & & & & & & 96 & : & & & & & & & & & 18 \\
\hline & & & & & & & & & 37 & : & & & & & & & & & 18 \\
\hline & & & & & & & & & & : & & & & & & & & & \\
\hline \multirow[t]{2}{*}{1546} & 1154 & 1765 & 1424 & 2034 & 1187 & 1644 & 1655 & 1770 & 1853 & : 685 & 482 & 758 & 630 & 881 & 517 & 723 & 729 & 775 & 772 \\
\hline & & & & & & & & & & : & & & & & & & & & \\
\hline \multirow[t]{2}{*}{2948} & 1864 & 2631 & 1340 & 2862 & 1732 & 2573 & 2483 & 2434 & 2748 & : $\quad 367$ & 338 & 478 & 243 & 518 & 314 & 487 & 451 & 441 & 499 \\
\hline & & & & & & & & & & : & & & & & & & & & \\
\hline 1594 & 18 & 96 & 2764 & 4896 & 19 & 4217 & 4138 & 4264 & 4661 & .632 & 826 & 12 & 873 & 399 & 831 & 90 & 1170 & 1217 & 1271 \\
\hline
\end{tabular}

KEANUNEE

ST LUCIE 2

LIMERICX 1

SALEN 1

BROWNS FERRY3

DRESDEN 3

HATCH 112

YOGTLE $1 \mathrm{L2}$

SUSQUEHANMA 112

GINNA

RANCHO SECO 1

TURKEY PT 112

CIINTON 1

NNP-1

INP-3

PALO VERDE 1

PALO VERDE 2

PALO VERDE 3

QUAD CITIES 112

LIMERICK 2

SEABROOK 1

SALEN 2

SUMNER 1

CRYSTAL RVR 3

FARLEY 1

RVR BEND 1

VATERFORD 3

HOPE CRK 1

BELEONTE 1

SEABROOK-2

B日LIEONTE 2

COMANCHE PK $1 \$ 2$

MCQUIRE 1

NINE MILE PT2

CALLATAY 1

PUR TOTAL

BNR TOTAL

TOTAL

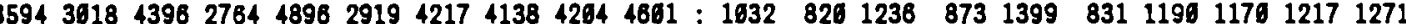


TABLE A.5. Middle Case (with Extended Burnup) Maximum At-Reactor Capacity-Projected Annual Storage Requirements (contd)

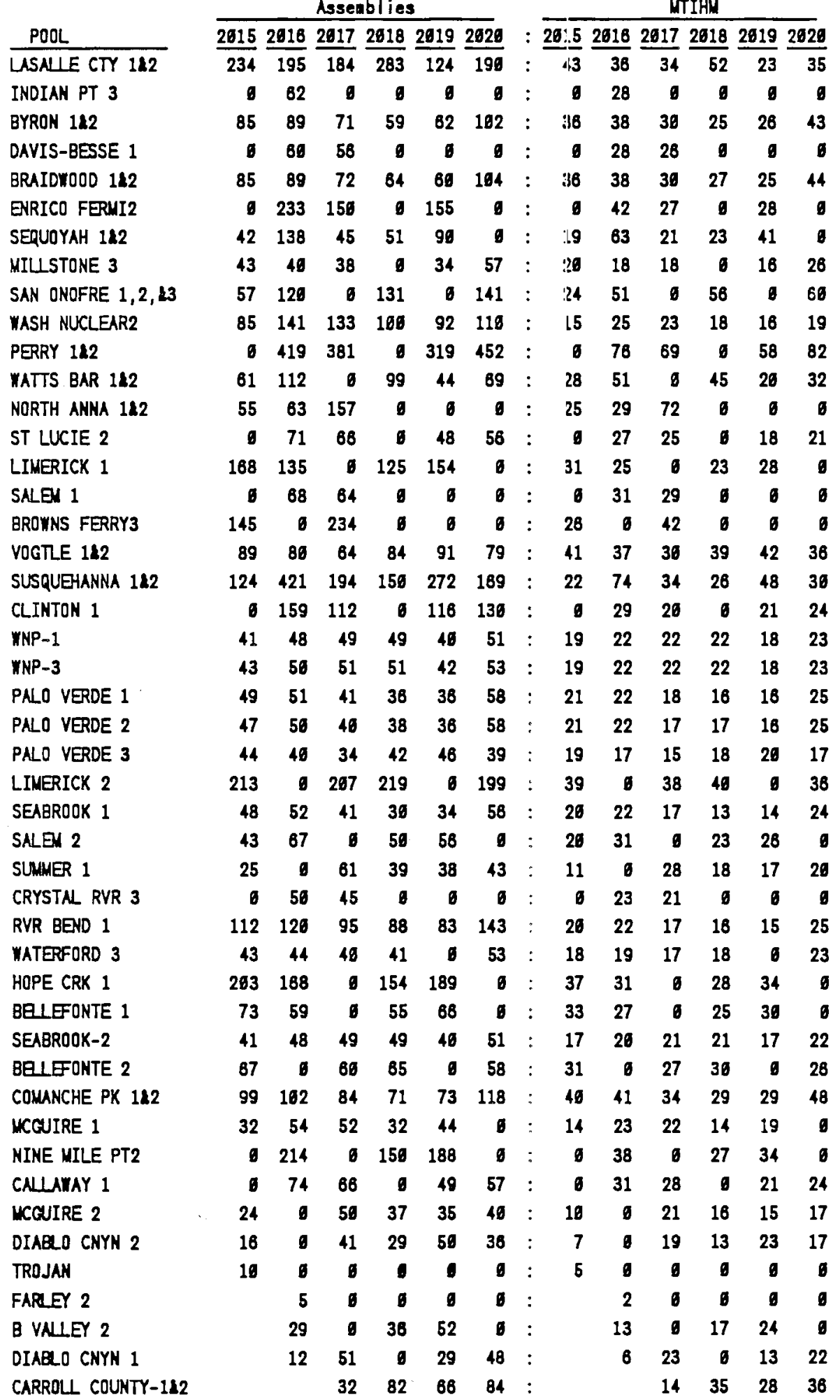


TABLE A.5. Middle Case (with Extended Burnup) Maximum At-Reactor Capacity-Projected Annual Storage Requirements (contd)

Assenblies

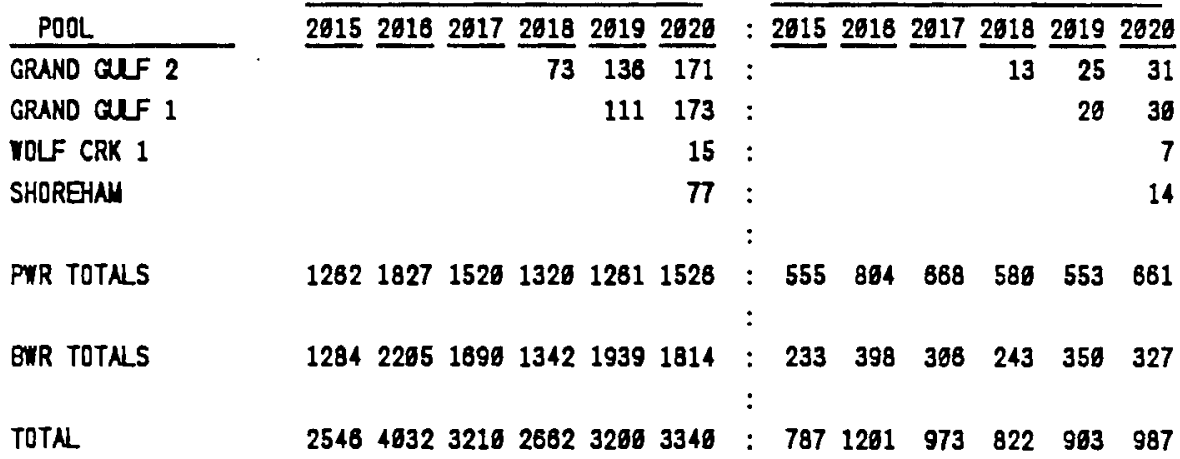


TABLE A.6. Middle Case (with Extended Burnup) Maximum At-Reactor Capacity-Projected Cumulative Storage Requirements

ASSEMEL IES

POOL

MIUSTONE 2

SURRY $1 \& 2$

MILSTONE 1

PALISADES

OCONEE 112

ST LUCIE 1

PEACHBOTTOM 2

PEACHBOTTOM 3

ROBINSON 2

LASALE CTY $1 \mathrm{H2}$

BRUNSWICK 2

PRAIRIE ISL $1 / 2$

PILGRIM 1

CALVERT CLF $1 \mathrm{L2}$

LACRASSE

OCONEE 3

FITZPATRICX

PWR TOTAL

BWR TOTAL

TOTAL

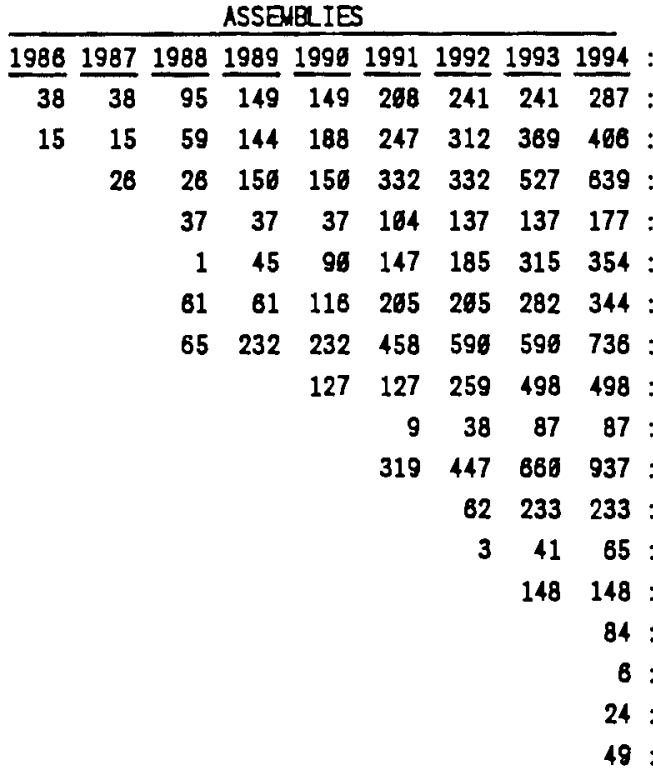

$\begin{array}{lllllllll}53 & 53 & 253 & 436 & 580 & 920 & 1121 & 1472 & 1828\end{array}$

$\begin{array}{lllllllll}22 & 22 & 99 & 180 & 242 & 376 & 483 & 612 & 753\end{array}$

$\begin{array}{llllllll}26 & 91 & 382 & 569 & 1236 & 1698 & 2656 & 3246\end{array}$ :

$\begin{array}{llllllll}5 & 18 & 69 & 92 & 224 & 388 & 483 & 596\end{array}$

$\begin{array}{lllllllll}53 & 79 & 344 & 818 & 1689 & 2158 & 28114128 & 5674\end{array}$

$\begin{array}{lllllllll}22 & 27 & 116 & 249 & 334 & 606 & 771 & 1694 & 1343\end{array}$ 
TABLE A.6. Middle Case (with Extended Burnup) Maximum At-Reactor Capacity-Projected Cumulative Storage Requirements (contd)

\begin{tabular}{|c|c|c|c|c|c|c|c|c|c|c|c|c|c|c|c|c|c|c|c|c|c|}
\hline \multirow[b]{2}{*}{ POOL } & \multicolumn{10}{|c|}{ ASSENBLIES } & \multicolumn{10}{|c|}{ HM } & \multirow[b]{2}{*}{20604} \\
\hline & 1995 & $\underline{1996}$ & $\underline{1997}$ & $\underline{1998}$ & $\underline{1999}$ & 2060 & 2001 & 2002 & 2063 & 2004 & $:$ & $\underline{1995}$ & $\underline{1998}$ & 1997 & $\underline{1998}$ & $\underline{1999}$ & 2000 & 2001 & 2002 & $\underline{2003}$ & \\
\hline NILLSTONE 2 & 338 & 336 & 465 & 454 & 454 & 512 & 547 & 547 & 585 & 646 & . & 137 & 137 & 184 & 184 & 184 & 286 & 222 & 222 & 237 & 262 \\
\hline SURRY 112 & 568 & 543 & 867 & 879 & 720 & 777 & 848 & 911 & 947 & 1841 & $:$ & 233 & 249 & 279 & 312 & 330 & 357 & 389 & 418 & 435 & 478 \\
\hline MIUSTONE 1 & 639 & 749 & 749 & 878 & 876 & 1938 & 1038 & 1246 & 1353 & 1353 & : & 114 & 133 & 133 & 155 & 155 & 184 & 184 & 221 & 240 & 246 \\
\hline PALISADES & 241 & 275 & 275 & 316 & 361 & 381 & 395 & 481 & 481 & 514 & : & 94 & 167 & 167 & 123 & 141 & 141 & 154 & 179 & 179 & 280 \\
\hline OCONEE $1 \mathrm{L2}$ & 411 & 488 & 556 & 596 & 641 & 787 & 745 & 877 & 915 & 987 & $:$ & 191 & 228 & 258 & 278 & 297 & 328 & 345 & 407 & 424 & 448 \\
\hline ST LUCIE 1 & 413 & 413 & 589 & 581 & 561 & 842 & 894 & 894 & 758 & 821 & $:$ & 139 & 139 & 168 & 188 & 188 & 213 & 232 & 232 & 252 & 276 \\
\hline PEACHBOTTON 2 & 938 & 936 & 1183 & 1327 & 1327 & 1547 & 1898 & 1898 & 1835 & 2623 & $:$ & 172 & 172 & 218 & 245 & 245 & 288 & 312 & 312 & 339 & 374 \\
\hline PEACHBOTTON 3 & 784 & 847 & 847 & 995 & 1170 & 1178 & 1312 & 1576 & 1578 & 1768 & : & 128 & 155 & 155 & 182 & 214 & 214 & 239 & 287 & 287 & 321 \\
\hline ROBINSON 2 & 129 & 159 & 213 & 244 & 244 & 292 & 323 & 378 & 498 & 406 & : & 55 & 68 & 92 & 185 & 185 & 128 & 139 & 182 & 175 & 175 \\
\hline LASALE CTY 112 & 1127 & 1253 & 1713 & 1846 & 2992 & 2417 & 2549 & 2779 & 3637 & 3213 & : & 265 & 228 & 312 & 336 & 365 & 440 & 484 & 506 & 553 & 585 \\
\hline BRUNSWICK 2 & 381 & 482 & 482 & 588 & 715 & 715 & 868 & 991 & 991 & 1132 & 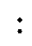 & 71 & 96 & 96 & 110 & 134 & 134 & 151 & 185 & 185 & 212 \\
\hline PRAIRIE ISL 112 & 138 & 188 & 282 & 312 & 346 & 415 & 440 & 522 & 572 & 834 & : & 45 & 63 & 91 & 189 & 118 & 144 & 153 & 182 & 200 & 221 \\
\hline PILGRIM 1 & 395 & 411 & 411 & 528 & 528 & 788 & 786 & 981 & 981 & 1655 & : & 54 & 73 & 73 & 93 & 93 & 125 & 125 & 168 & 180 & 187 \\
\hline CNLVERT CLF $1 \mathbf{2} 2$ & 142 & 198 & 338 & 386 & 439 & 573 & 623 & 689 & 797 & 856 & $:$ & 56 & 75 & 133 & 152 & 172 & 225 & 245 & 275 & 313 & 338 \\
\hline LACROSSE & 28 & 39 & 64 & 78 & 95 & 116 & 129 & 153 & 186 & 186 & : & 3 & 4 & 7 & 8 & 10 & 13 & 14 & 17 & 18 & 18 \\
\hline OCONEE 3 & 88 & 80 & 152 & 152 & 199 & 199 & 237 & 369 & 309 & 383 & : & 37 & 37 & 78 & 70 & 92 & 92 & - 110 & 143 & 143 & 188 \\
\hline ITZPATRICK & 192 & 192 & 367 & 474 & 474 & 634 & 742 & 742 & 848 & 985 & : & 35 & 35 & 68 & 85 & 85 & 114 & 133 & 133 & 152 & 177 \\
\hline BRUNSWICK 1 & 163 & 103 & 287 & 394 & 394 & 560 & 653 & 653 & 751 & 892 & : & 19 & 19 & 54 & 74 & 74 & 165 & 122 & 122 & 148 & 167 \\
\hline INDIAN PT 3 & 2 & 2 & 81 & 136 & 136 & 294 & 294 & 293 & 336 & 336 & : & 1 & 1 & 37 & 82 & 82 & 93 & 93 & 134 & 153 & 153 \\
\hline BYRON 112 & & 28 & 122 & 211 & 281 & 378 & 437 & 545 & 695 & 693 & : & & 11 & 52 & 89 & 119 & 160 & 185 & 238 & 258 & 293 \\
\hline COOPER STH & & 84 & 182 & 251 & 331 & 438 & 584 & 823 & 891 & 778 & : & & 12 & 33 & 48 & 88 & 79 & 92 & 114 & 128 & 142 \\
\hline FORT CALHOUN & & 12 & 53 & 53 & 92 & 137 & 137 & 178 & 203 & 263 & : & & 4 & 19 & 19 & 33 & 49 & 49 & 64 & 73 & 73 \\
\hline DAYIS-BESSE 1 & & 25 & 25 & 65 & 113 & 113 & 156 & 221 & 221 & 274 & $:$ & & 12 & 12 & 30 & 53 & 53 & 76 & 103 & 163 & 128 \\
\hline BIG ROCK 1 & & & 6 & 28 & 37 & 55 & 76 & 76 & 70 & 78 & : & & & 1 & 3 & 5 & 7 & 9 & 9 & 9 & 9 \\
\hline POINT BEACH 112 & & & 39 & 83 & 128 & 186 & 236 & 299 & 343 & 395 & : & & & 14 & 29 & 45 & 68 & 82 & 186 & 122 & 146 \\
\hline ARK NULCLEAR 1 & & & & 46 & 46 & 163 & 163 & 174 & 174 & 228 & : & & & & 19 & 19 & 48 & 48 & 81 & 81 & 168 \\
\hline BRAIDTOOD 112 & & & & 86 & 151 & 248 & 368 & 416 & 476 & 563 & & & & & 34 & 64 & 165 & 130 & 178 & 261 & 238 \\
\hline INDIAN PT 2 & & & & 35 & 88 & 86 & 127 & 261 & 201 & 258 & : & & & & 16 & 39 & 39 & 58 & 92 & 92 & 118 \\
\hline ENRICO FERMI2 & & & & 224 & 224 & 473 & 473 & 744 & 962 & 992 & : & & & & 41 & 41 & 86 & 86 & 135 & 164 & 164 \\
\hline 8 VAШEY 1 & & & & 4 & 57 & 57 & 94 & 172 & 172 & 231 & : & & & & 2 & 28 & 28 & 43 & 79 & 79 & 168 \\
\hline OYSTER CRK 1 & & & & 41 & 41 & 197 & 363 & 363 & 461 & 461 & : & & & & 7 & 7 & 35 & 54 & 54 & 71 & 71 \\
\hline SERUOYAH 112 & & & & 1 & 58 & 265 & 253 & 334 & 431 & 493 & & & & & & 27 & 94 & 118 & 153 & 198 & 226 \\
\hline ZION 122 & & & & & 39 & 129 & 185 & 287 & 341 & 417 & & & & & & 18 & 59 & 85 & 131 & 158 & 191 \\
\hline MAINE YANKEE & & & & & 19 & 85 & 85 & 159 & 262 & 262 & & & & & & 7 & 32 & 32 & 68 & 76 & 76 \\
\hline BROWNS FERRY2 & & & & & 81 & 81 & 243 & 243 & 461 & 401 & & & & & & 15 & 15 & 44 & 44 & 73 & 73 \\
\hline $\operatorname{coOK} 112$ & & & & & & 91 & 139 & 236 & 334 & 399 & & & & & & & 37 & 59 & 96 & 148 & 170 \\
\hline MILLSTONE 3 & & & & & & 14 & 51 & 51 & 96 & 133 & & & & & & & 8 & 24 & 24 & 42 & 61 \\
\hline SAN ONOFRE $1,2,13$ & & & & & & 147 & 263 & 263 & 381 & 451 & & & & & & & 55 & 104 & 184 & 154 & 184 \\
\hline WASH MUCLEAR2 & & & & & & & 51 & 216 & 367 & 435 & & & & & & & & 9 & 38 & 54 & 77 \\
\hline ARK NUCLEAR 2 & & & & & & & & 34 & 34 & 90 & & & & & & & & & 14 & 14 & 37 \\
\hline PERRY 112 & & & & & & & & 372 & 372 & 816 & & & & & & & & & 68 & 68 & 148 \\
\hline DRESDEX 2 & & & & & & & & 111 & 111 & 264 & & & & & & & & & 20 & 20 & 47 \\
\hline HADDAU NECK & & & & & & & & 38 & 64 & 64 & & & & & & & & & 15 & 26 & 26 \\
\hline DUANE ARNOLD & & & & & & & & 44 & 169 & 169 & & & & & & & & & 8 & 20 & 26 \\
\hline NINE MILE PT1 & & & & & & & & 86 & 86 & 244 & & & & & & & & & 15 & 15 & 43 \\
\hline BRDWNS FERRY1 & & & & & & & & 119 & 119 & 342 & & & & & & & & & 22 & 22 & 62 \\
\hline WATTS BAR 112 & & & & & & & & 96 & 137 & 198 & & & & & & & & & 41 & 63 & 91 \\
\hline
\end{tabular}



TABLE A.6. Middle Case (with Extended Burnup) Maximum At-Reactor Capacity--
Projected Cumulative Storage Requirements (contd)

ASSENBLIES

MTIHM

POOL

VT YANKEE 1

NORTH ANNA 112

KEUAUNEE

ST LUCIE 2

LIMERICK 1

SALEN 1

PHR TOTAL

BWR TOTAL

TOTAL
$199519981997199819992000200120002200320004: 199519961997199819992000200012002200320004$

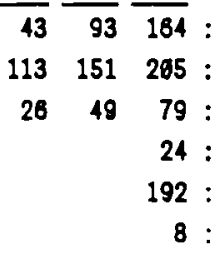

$2394 \quad \begin{array}{cccccc}3637 & 5159 & 7618 & 10695 & \\ 2731 & & 4468 & 6881 & 9518 & 12192\end{array}$

$4413 \quad \begin{array}{llllll}6291 & & 8287 & 11271 & 15114 & :\end{array}$

$988{ }_{1129} 1495 \quad 18199^{2139} 2755{ }^{3167} 39855^{4477} 5121$

$801{ }_{929}^{1141} 1384^{1501}{ }^{2039}{ }^{2939} 2477^{2733} 3201$

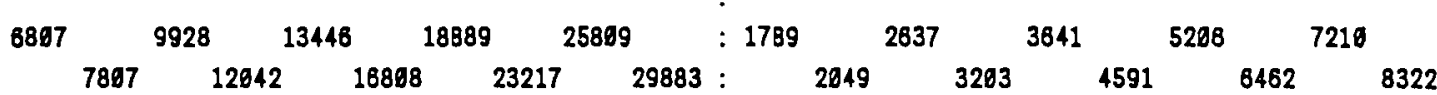


TABLE A.6. Middle Case (with Extended Burnup) Maximum At-Reactor Capacity-Projected Cumulative Storage Requirements (contd)

POOL MILSTONE 2 SURRY $1 \mathbf{1 2}$

MILSTONE 1

PNLISADES

OCONEE 112

ST LUCIE 1

PEACHBOTTOM 2

PEACHBOTTOM 3

ROBINSON 2

LASALIE CTY $1 \& 2$

BRUNSWICK 2

PRAIRIE ISL $1 \& 2$

PILGRIM 1

CALVERT CLF $1 \& 2$

LACROSSE

OCONEE 3

FITZPATRICK

BRUNSWICK 1

INDIAN PT 3

BYRON 112

COOPER STN

FORT CALHOUN

DAVIS-BESSE 1

BIG ROCK 1

POINT BEACH $1 \mathbf{2}$

ARK NUCLEAR 1

BRAIDWOOD 112

INDIAN PT 2

ENRICO FERMI2

8 VALLEY 1

OYSTER CRK 1

SEQUDYAH $1 \& 2$

ZION 122

MAINE YANKEE

BROWNS FERRY2

COOK 112

MILSTONE 3

SAN ONOFRE $1,2,13$

IASH NUCLEAR2

ARK NUCLEAR 2

PERRY $1: 2$

DRESDEN 2

HADDN NECK
ASSEMBLIES

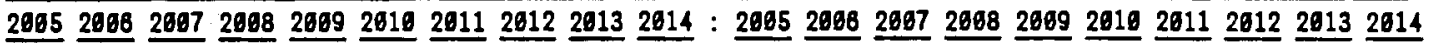

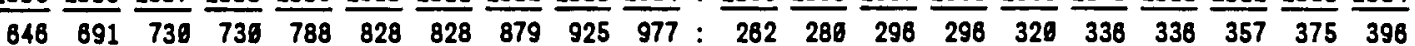

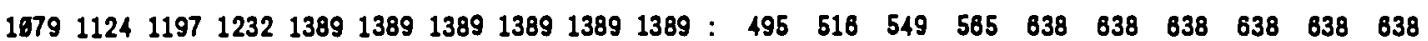

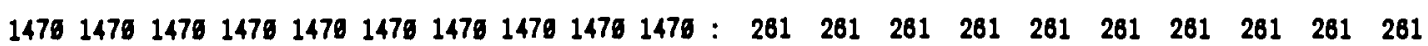
$\begin{array}{lllllllllllllllllllll}554 & 554 & 693 & 603 & 603 & 603 & 603 & 693 & 663 & 663 & : & 216 & 216 & 235 & 235 & 235 & 235 & 235 & 235 & 235 & 235\end{array}$ $\begin{array}{lllllllllllllllllllll}1013 & 1961 & 1146 & 1177 & 1354 & 1354 & 1354 & 1354 & 1354 & 1354 & \text { : } & 476 & 492 & 529 & 548 & 628 & 628 & 628 & 628 & 628 & 628\end{array}$ $\begin{array}{llllllllllllllllllllll}821 & 887 & 938 & 938 & 1011 & 1082 & 1082 & 1130 & 1187 & 1187 & : & 276 & 297 & 316 & 318 & 339 & 358 & 358 & 380 & 461 & 461\end{array}$

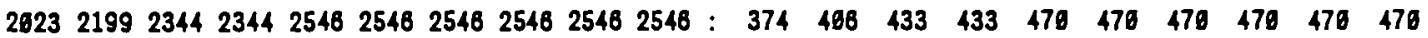

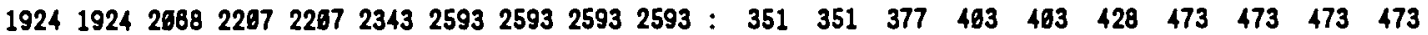
$\begin{array}{lllllllllllllllllllll}439 & 477 & 477 & 477 & 477 & 477 & 477 & 477 & 477 & 477 & 0 & 189 & 265 & 265 & 265 & 265 & 265 & 265 & 265 & 265 & 265\end{array}$

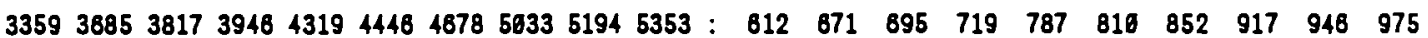

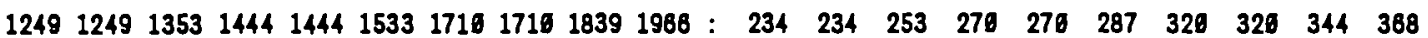
$\begin{array}{lllllllllllllllllllll}687 & 716 & 765 & 789 & 942 & 942 & 942 & 942 & 942 & 942 & : & 246 & 249 & 286 & 275 & 330 & 336 & 336 & 336 & 336 & 336\end{array}$

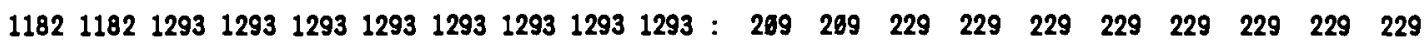
\begin{tabular}{lllllllllll|llllllllll}
964 & 1011 & 1659 & 1167 & 1228 & 1278 & 1352 & 1454 & 1588 & 1725 & 355 & 397 & 418 & 435 & 482 & 501 & 531 & 571 & 592 & 647
\end{tabular} $\begin{array}{lllllllllllllllllllll}168 & 168 & 168 & 168 & 168 & 168 & 168 & 168 & 168 & 168 & : & 18 & 18 & 18 & 18 & 18 & 18 & 18 & 18 & 18 & 18\end{array}$ $\begin{array}{lllllllllllllllllllll}468 & 468 & 448 & 448 & 596 & 598 & 508 & 598 & 568 & 508 & : & 189 & 189 & 298 & 268 & 235 & 235 & 235 & 235 & 235 & 235\end{array}$

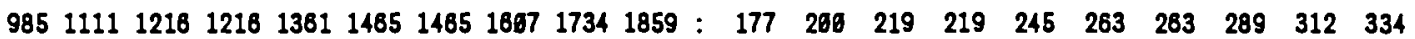

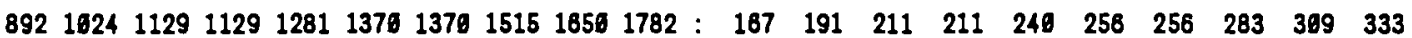
$\begin{array}{lllllllllllllllllllll}391 & 391 & 446 & 498 & 498 & 545 & 545 & 604 & 658 & 658 & : & 178 & 178 & 203 & 228 & 228 & 249 & 249 & 275 & 369 & 380\end{array}$ $\begin{array}{lllllllllllllllllllll}781 & 845 & 925 & 983 & 1868 & 1132 & 1263 & 1364 & 1398 & 1454 & : & 336 & 357 & 391 & 416 & 452 & 479 & 589 & 551 & 591 & 615\end{array}$ $\begin{array}{lllllllllllllllllllll}778 & 778 & 778 & 778 & 778 & 778 & 778 & 778 & 778 & 778 & 142 & 142 & 142 & 142 & 142 & 142 & 142 & 142 & 142 & 142\end{array}$ $\begin{array}{lllllllllllllllllllll}241 & 281 & 281 & 311 & 311 & 311 & 311 & 311 & 311 & 311 & : & 87 & 101 & 101 & 112 & 112 & 112 & 112 & 112 & 112 & 112\end{array}$ $\begin{array}{lllllllllllllllllllll}318 & 318 & 357 & 394 & 394 & 436 & 499 & 499 & 549 & 598 & : & 149 & 149 & 187 & 184 & 184 & 201 & 234 & 234 & 257 & 280\end{array}$ $\begin{array}{lllllllllllllllllllll}70 & 70 & 76 & 76 & 76 & 76 & 76 & 76 & 70 & 76: & 9 & 9 & 9 & 9 & 9 & 9 & 9 & 9 & 9 & 9\end{array}$ $\begin{array}{lllllllllllllllllllll}437 & 484 & 628 & 628 & 628 & 628 & 628 & 628 & 628 & 628 & : & 155 & 172 & 222 & 222 & 222 & 222 & 222 & 222 & 222 & 222\end{array}$ $\begin{array}{lllllllllllllllllllll}273 & 273 & 314 & 314 & 371 & 371 & 371 & 371 & 371 & 371 & : & 127 & 127 & 146 & 146 & 172 & 172 & 172 & 172 & 172 & 172\end{array}$ $\begin{array}{lllllllllllllllllllll}652 & 715 & 795 & 855 & 941 & 1685 & 1678 & 1179 & 1275 & 1335 & : & 278 & 362 & 336 & 361 & 398 & 425 & 456 & 498 & 539 & 564\end{array}$ $\begin{array}{lllllllllllllllllllll}368 & 368 & 348 & 389 & 389 & 389 & 389 & 389 & 389 & 389 & : & 146 & 140 & 180 & 179 & 179 & 179 & 179 & 179 & 179 & 179\end{array}$ $\begin{array}{llllllllllllllllllllll}1128 & 1128 & 1336 & 1336 & 1554 & 1761 & 1761 & 1960 & 1980 & 2112 & : & 265 & 265 & 242 & 242 & 283 & 310 & 310 & 357 & 357 & 384\end{array}$ $\begin{array}{lllllllllllllllllllll}279 & 279 & 322 & 358 & 358 & 394 & 469 & 469 & 522 & 574 & : & 129 & 129 & 148 & 185 & 185 & 182 & 216 & 216 & 241 & 285\end{array}$ $\begin{array}{lllllllllllllllllllll}525 & 525 & 525 & 525 & 525 & 525 & 525 & 525 & 525 & 525 & : & 93 & 93 & 93 & 93 & 93 & 93 & 93 & 93 & 93 & 93\end{array}$ $\begin{array}{lllllllllllllllllllll}545 & 663 & 650 & 745 & 812 & 858 & 1026 & 1683 & 1143 & 1201 & : & 256 & 277 & 298 & 342 & 373 & 394 & 468 & 497 & 525 & 551\end{array}$ $\begin{array}{lllllllllllllllllllll}482 & 554 & 669 & 863 & 898 & 896 & 898 & 898 & 898 & 896 & : & 226 & 253 & 278 & 303 & 410 & 410 & 410 & 410 & 410 & 410\end{array}$ $\begin{array}{lllllllllllllllllllll}248 & 306 & 360 & 360 & 300 & 360 & 300 & 360 & 360 & 300 & : & 93 & 112 & 112 & 112 & 112 & 112 & 112 & 112 & 112 & 112\end{array}$ $\begin{array}{lllllllllllllllllllll}585 & 585 & 750 & 750 & 986 & 986 & 986 & 1221 & 1221 & 1437 & : & 108 & 168 & 138 & 136 & 179 & 179 & 179 & 222 & 222 & 261\end{array}$ $\begin{array}{lllllllllllllllllllll}458 & 518 & 823 & 878 & 745 & 801 & 971 & 1032 & 1698 & 1291 & : & 193 & 221 & 287 & 289 & 328 & 343 & 418 & 444 & 471 & 554\end{array}$ $\begin{array}{llllllllllllllllllllll}185 & 222 & 287 & 364 & 365 & 468 & 466 & 458 & 512 & 545 & : & 85 & 162 & 123 & 146 & 168 & 185 & 185 & 211 & 236 & 252\end{array}$ $\begin{array}{lllllllllllllllllllll}511 & 577 & 839 & 898 & 771 & 829 & 922 & 995 & 1662 & 1128 & : & 218 & 238 & 285 & 289 & 321 & 348 & 385 & 417 & 445 & 473\end{array}$ $\begin{array}{lllllllllllllllllllll}546 & 857 & 751 & 842 & 978 & 1084 & 1227 & 1355 & 1471 & 1588 & : & 95 & 118 & 132 & 148 & 172 & 187 & 218 & 239 & 259 & 279\end{array}$ $\begin{array}{lllllllllllllllllllll}139 & 139 & 188 & 188 & 248 & 248 & 324 & 383 & 383 & 383 & : & 58 & 58 & 78 & 78 & 163 & 163 & 135 & 180 & 168 & 168\end{array}$

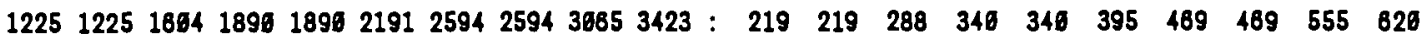
$\begin{array}{lllllllllllllllllllll}392 & 392 & 392 & 392 & 392 & 392 & 392 & 392 & 392 & 392 & : & 70 & 76 & 70 & 76 & 76 & 76 & 70 & 70 & 76 & 76\end{array}$

$\begin{array}{lllllllllllllllllllll}99 & 139 & 139 & 139 & 139 & 139 & 139 & 139 & 139 & 139 & : & 41 & 57 & 57 & 57 & 57 & 57 & 57 & 57 & 57 & 57\end{array}$ 
TABLE A.6. Middle Case (with Extended Burnup) Maximum At-Reactor Capacity-Projected Cumulative Storage Requiremeits (contd)

ASSEMBLIES

POOL

DUANE ARNOLD

NINE MILE PT1

BROWNS FERRY1

VATTS BAR $1 \mathbf{2 2}$

VT YANKEE 1

NORTH ANNA 112

KETAUNEE

ST LUCIE 2

LIMERICK 1

SALEM 1

BROYNS FERRY3

DRESDEN 3

HATCH 122

VOGRE $1 \$ 2$

SUSQUEHANNA 182

GINNA

RANCHO SECO 1

TURKEY PT $1 \mathbf{2 2}$

CLINTON 1

WNP-1

WNP-3

PALO VERDE 1

PALO VERDE 2

PALO VERDE 3

QUAD CITIES $1: 2$

LIMERICK 2

SEABROOK 1

SALEX 2

SUMMER 1

CRYSTAL RVR 3

FARLEY 1

RVR BEND 1

WATERFORD 3

HOPE CRK 1

BELEONTE 1

SEABROOX-2

B日LEONTE 2

COMANCHE PK $1: 2$

UCOUIRE 1

NIHE MILE PT2

CALATAY 1

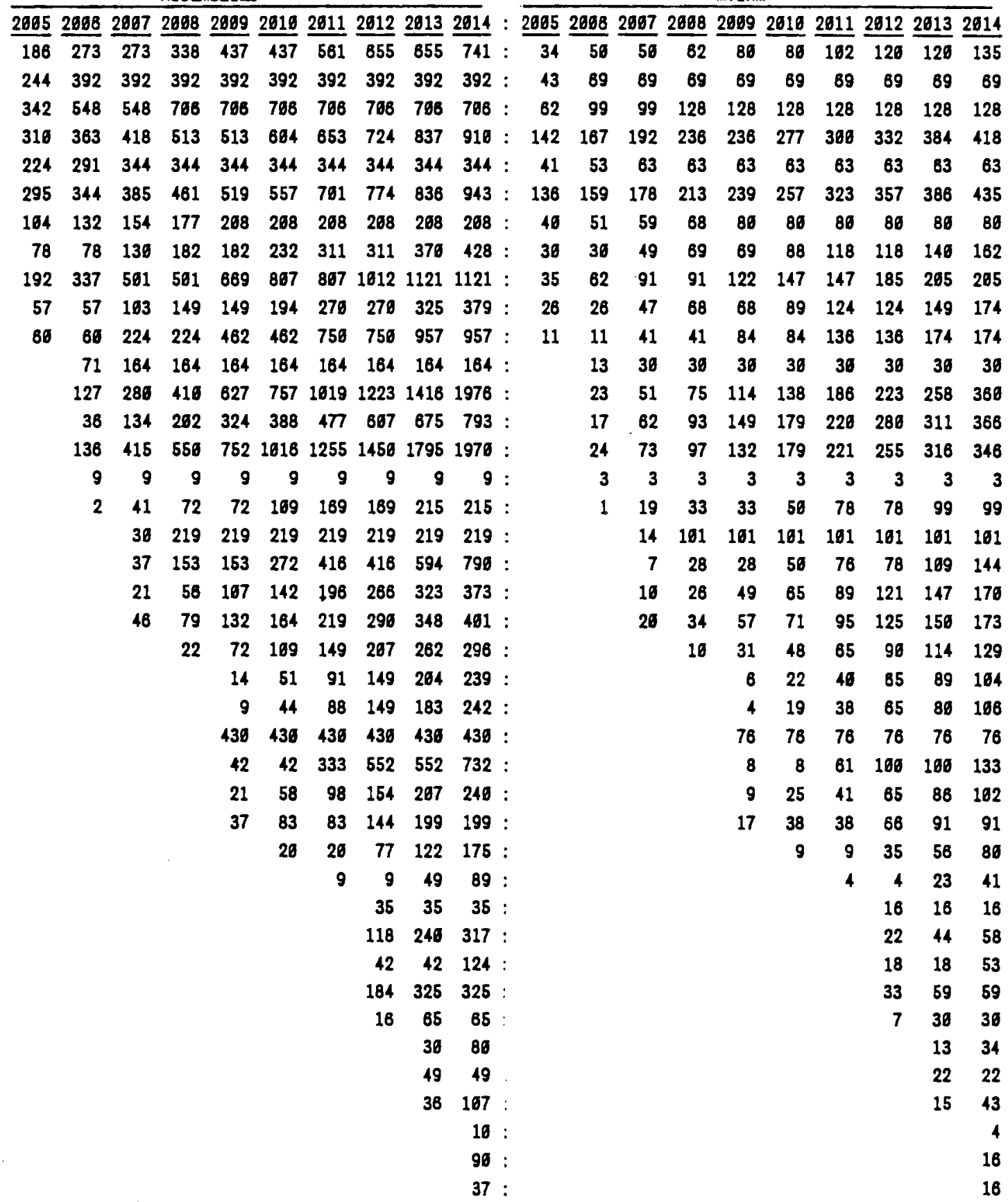


TABLE A.6. Middle Case (with Extended Burnup) Maximum At-Reactor Capacity-Projected Cumulative Storage Requirements (contd)

ASSENQLIES

NTIHM

POOL $\underline{2005} 20006$ 2007 2008 2009 $201020112012 \underline{2013} 2014: 2005$ 2006 20072008200920102011201220132014

PUR TOTAL

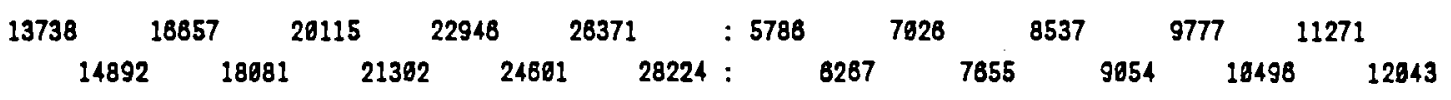

BWR TOTAL

$\begin{array}{cccccccccccc}19739 & 24235 & 28438 & 32741 & 37658 & : 3568 & 4384 & 5145 & 5928 & 8818 \\ 21683 & 25574 & 30168 & 35224 & 48486 & : & 3996 & 4627 & 5459 & 6376 & 7317\end{array}$

TOTAL

$\begin{array}{llllllllllllllll}33477 & 46891 & 48551 & 55887 & 64029 & : & 9354 & 11410 & 13681 & 15782 & 18989 \\ 36495 & 43855 & 51470 & 59825 & 68630 & : & 10174 & 12282 & 14513 & 16873 & 19361\end{array}$


TABLE A.6. Middle Case (with Extended Burnup) Maximum At-Reactor Capacity-Projected Cumulative Storage Requirements (contd)

ASSEMBLIES

POOL

MILISTONE 2

SURRY 112

MIUSTONE 1

PALISADES

OCONEE 112

ST LUCIE 1

PEACHBOTTOM 2

PEACHBOTTOM 3

ROBINSON 2

LASAUE CTY 112

BRUNSWICK 2

PRAIRIE ISL $1: 2$

PILGRIU 1

CALVERT CLF $1: 2$

LACROSSE

OCONEE 3

FITZPATRICK

BRUNSWICK 1

INDIAN PT 3

BYRON $1: 2$

COOPER STN

FORT CALHOUN

DAYIS-BESSE 1

BIG ROCK 1

POINT BEACH 122

ARK NUCLEAR 1

BRAIDYOOD 112

INDIAN PT 2

ENRICO FERMI2

B VALEY 1

OYSTER CRK 1

SERUOYAH 122

ZION 112

MAINE YANKEE

BROWNS FERRY2

COOK 112

MIULSTONE 3

SAN ONOFRE $1,2,13$

WASH MUCLEAR2

ARK NUCLEAR 2

PERRY 112

DRESDEN 2

HADDN NECX

DUANE ARNOLD

NINE MILE PT1
MTIHM

$\overline{2015} 2016$ 2017 2018 2019 2026 :2015 2016 2017 2018 2019 2026

$\frac{777}{977} \frac{977}{977} \frac{777}{977}: \frac{393}{396} \frac{396}{398} \frac{398}{398}$

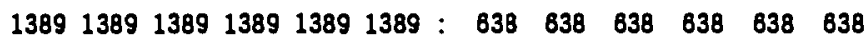

$\begin{array}{llllllllllll}1470 & 1470 & 1470 & 1470 & 1470 & 1470: & 261 & 261 & 261 & 261 & 281 & 281\end{array}$

$\begin{array}{lllllllllllll}603 & 603 & 603 & 603 & 603 & 603 & : & 235 & 235 & 235 & 235 & 235 & 235\end{array}$

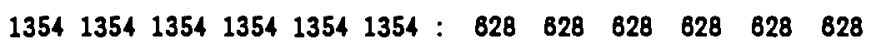

$\begin{array}{lllllllllllll}1187 & 1187 & 1187 & 1187 & 1187 & 1187 & \text { : } & 401 & 401 & 401 & 401 & 461 & 461\end{array}$

$\begin{array}{llllllllllllll}2546 & 2546 & 2546 & 2546 & 2546 & 2546 & : & 470 & 470 & 470 & 470 & 470 & 470\end{array}$

$\begin{array}{llllllllllllll}2593 & 2593 & 2593 & 2593 & 2593 & 2593 & : & 473 & 473 & 473 & 473 & 473 & 473\end{array}$

$\begin{array}{lllllllllllll}477 & 477 & 477 & 477 & 477 & 477 & : & 285 & 205 & 205 & 205 & 265 & 205\end{array}$

$\begin{array}{llllllllllllll}5587 & 5782 & 5968 & 6249 & 6373 & 6563 & : & 1018 & 1053 & 1087 & 1138 & 1161 & 1195\end{array}$

$\begin{array}{lllllllllllll}1988 & 1968 & 1966 & 1968 & 1966 & 1966 & : & 36.8 & 368 & 368 & 368 & 368 & 368\end{array}$

$\begin{array}{lllllllllllll}942 & 942 & 942 & 942 & 942 & 942 & : & 356 & 330 & 330 & 330 & 330 & 330\end{array}$

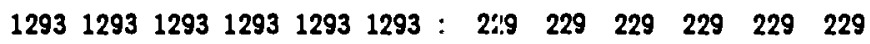

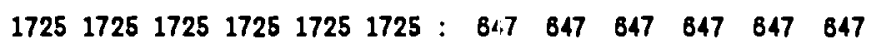

$\begin{array}{lllllllllllll}168 & 168 & 168 & 168 & 168 & 186 & : & .8 & 18 & 18 & 18 & 18 & 18\end{array}$

$\begin{array}{lllllllllllll}508 & 596 & 598 & 598 & 508 & 586 & : & 2315 & 235 & 235 & 235 & 235 & 235\end{array}$

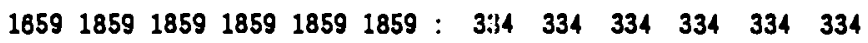

$\begin{array}{lllllllllllll}1782 & 1782 & 1782 & 1782 & 1782 & 1782 & : & 3.33 & 333 & 333 & 333 & 333 & 333\end{array}$

$\begin{array}{lllllllllllll}658 & 728 & 720 & 728 & 720 & 728 & : & 3116 & 328 & 328 & 328 & 328 & 326\end{array}$

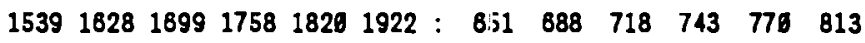

$\begin{array}{lllllllllllll}778 & 778 & 778 & 778 & 778 & 778 & : & 142 & 142 & 142 & 142 & 142 & 142\end{array}$

$\begin{array}{lllllllllllll}311 & 311 & 311 & 311 & 311 & 311 & : & 112 & 112 & 112 & 112 & 112 & 112\end{array}$

$\begin{array}{lllllllllllll}598 & 658 & 714 & 714 & 714 & 714 & : & 236 & 398 & 334 & 334 & 334 & 334\end{array}$

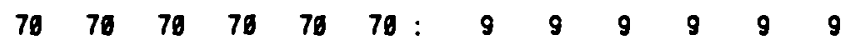

$\begin{array}{lllllllllllll}628 & 628 & 628 & 628 & 628 & 628 & : & 222 & 222 & 222 & 222 & 222 & 222\end{array}$

$\begin{array}{lllllllllllll}371 & 371 & 371 & 371 & 371 & 371 & : & 172 & 172 & 172 & 172 & 172 & 172\end{array}$

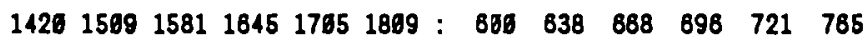

$\begin{array}{lllllllllllll}389 & 389 & 369 & 389 & 389 & 389 & : & 179 & 179 & 179 & 179 & 179 & 179\end{array}$

$\begin{array}{llllllllllll}2112 & 23452495249528502850: & 384 & 427 & 454 & 454 & 482 & 482\end{array}$

$\begin{array}{lllllllllllll}574 & 574 & 574 & 574 & 574 & 574 & 285 & 265 & 265 & 285 & 285 & 285\end{array}$

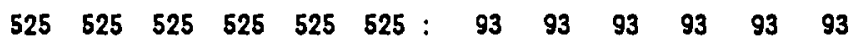

$\begin{array}{lllllllllllll}1243 & 1381 & 1428 & 1477 & 1587 & 1567 & : & 571 & 834 & 655 & 678 & 719 & 719\end{array}$

$\begin{array}{lllllllllllll}896 & 896 & 896 & 898 & 896 & 898 & : & 410 & 410 & 410 & 410 & 410 & 410\end{array}$

$\begin{array}{lllllllllllll}360 & 390 & 360 & 306 & 300 & 360 & : & 112 & 112 & 112 & 112 & 112 & 112\end{array}$

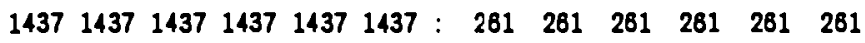

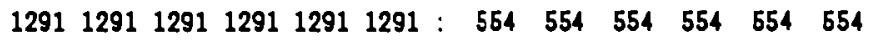

$\begin{array}{lllllllllllll}588 & 828 & 686 & 668 & 786 & 757 & : & 271 & 298 & 367 & 397 & 323 & 349\end{array}$

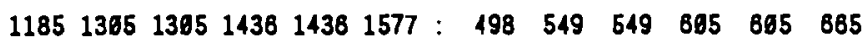

$\begin{array}{lllllllllllll}1871 & 1812 & 1945 & 2645 & 2137 & 2247 & : & 294 & 319 & 343 & 360 & 376 & 396\end{array}$

$\begin{array}{lllllllllllll}383 & 383 & 383 & 383 & 383 & 383 & : & 168 & 168 & 160 & 160 & 168 & 160\end{array}$

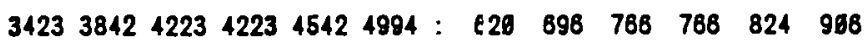

$\begin{array}{lllllllllllll}392 & 392 & 392 & 392 & 392 & 392 & : & 76 & 70 & 70 & 76 & 76 & 76\end{array}$

$\begin{array}{lllllllllllll}139 & 139 & 139 & 139 & 139 & 139 & : & 57 & 57 & 57 & 57 & 57 & 57\end{array}$

$\begin{array}{lllllllllllll}741 & 741 & 741 & 741 & 741 & 741 & : & 135 & 135 & 135 & 135 & 135 & 135\end{array}$

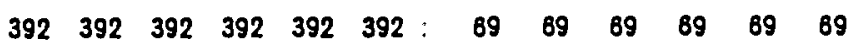


TABLE A.6. Middle Case (with Extended Burnup) Maximum At-Reactor Capacity-Projected Cumulative Storage Requirements (contd)

\begin{tabular}{|c|c|c|c|c|c|c|c|c|c|c|c|c|c|}
\hline \multirow[b]{2}{*}{ POOL } & \multicolumn{7}{|c|}{ ASSEMBLIES } & \multicolumn{6}{|c|}{ MTIHM } \\
\hline & $\underline{2615}$ & $\underline{2018}$ & $\underline{2017}$ & $\underline{2618}$ & 2019 & 2020 & & 2015 & $\underline{2016}$ & $\underline{2617}$ & 2618 & $\underline{2019}$ & $\underline{202}$ \\
\hline BROWNS FERRY1 & 766 & 786 & 786 & 796 & 706 & 796 & & 128 & 128 & 128 & 128 & 128 & 12 \\
\hline MATTS BAR 112 & 971 & 1983 & 1083 & 1182 & 1228 & 1295 & & 448 & 497 & 497 & 543 & 583 & 59 \\
\hline VT YANKEE 1 & 344 & 344 & 344 & 344 & 344 & 344 & & 63 & 63 & 83 & 83 & 63 & \\
\hline NORTH ANKA 112 & 998 & 1881 & 1218 & 1218 & 1218 & 1218 & & 460 & 490 & 582 & 562 & 582 & 56 \\
\hline KETAUNEE & 268 & 268 & 298 & 208 & 298 & 298 & & 80 & 80 & 88 & 86 & 80 & 8 \\
\hline T LUCIE 2 & 428 & 499 & 585 & 565 & 813 & 869 & & 182 & 189 & 214 & 214 & 233 & 25 \\
\hline IMERICK 1 & 1289 & 1424 & 1424 & 1549 & 1763 & 1703 & & 235 & 280 & 280 & 283 & 311 & 31 \\
\hline LEN 1 & 379 & 447 & 511 & 511 & 511 & 511 & & 174 & 265 & 235 & 235 & 235 & 23 \\
\hline RONNS FERRY3 & 1162 & 1162 & 1336 & 1336 & 1336 & 1336 & & 260 & 200 & 243 & 243 & 243 & 24 \\
\hline RESDEN 3 & 184 & 164 & 164 & 164 & 164 & 164 & & 39 & 39 & 36 & 30 & 36 & \\
\hline HATCH $1: 2$ & 1978 & 1978 & 1978 & 1976 & 1976 & 1978 & & 360 & 380 & 386 & 380 & 360 & 380 \\
\hline DGTLE $1: 2$ & 882 & 962 & 1628 & 1116 & 1261 & 1280 & & 467 & 444 & 473 & 512 & 554 & 591 \\
\hline SUSQUEEHANKA & 2694 & 2515 & 2769 & 2859 & 3131 & 3360 & & 368 & 442 & 476 & 503 & 556 & 58 \\
\hline GINMA & 9 & 9 & 9 & 9 & 9 & 9 & & 3 & 3 & 3 & 3 & 3 & \\
\hline INCHO SECO 1 & 215 & 215 & 215 & 215 & 215 & 215 & & 99 & 99 & 99 & 99 & 99 & 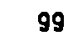 \\
\hline TURKEY PT 112 & 219 & 219 & 219 & 219 & 219 & 219 & & 161 & 101 & 101 & 161 & 161 & 161 \\
\hline ZINTON 1 & 790 & 949 & 1961 & 1061 & 1177 & 1367 & & 144 & 173 & 194 & 194 & 215 & 23 \\
\hline$N P-1$ & 414 & 462 & 511 & 566 & 898 & 651 & ; & 189 & 211 & 233 & 255 & 274 & 297 \\
\hline NP-3 & 444 & 494 & 545 & 598 & 838 & 891 & & 192 & 213 & 235 & 257 & 275 & 298 \\
\hline NLLO VERDE 1 & 345 & 398 & 437 & 473 & 599 & 587 & & 151 & 173 & 191 & 266 & 222 & 24 \\
\hline ALO VERDE 2 & 288 & 336 & 378 & 414 & 450 & 508 & & 125 & 147 & 184 & 181 & 196 & 222 \\
\hline 'NLO YERDE 3 & 288 & 328 & 360 & 402 & 448 & 487 & & 125 & 142 & 157 & 175 & 195 & 21 \\
\hline QUAD CITIES 122 & 436 & 430 & 430 & 430 & 439 & 430 & & 78 & 78 & 76 & 76 & 76 & 7 \\
\hline LIMERICK 2 & 945 & 946 & 1152 & 1371 & 1371 & 1570 & & 172 & 172 & 210 & 249 & 249 & 288 \\
\hline EABRDOK 1 & 288 & 340 & 381 & 411 & 445 & 591 & & 122 & 144 & 181 & 174 & 188 & 21 \\
\hline SALE 2 & 242 & 369 & 399 & 359 & 415 & 415 & & 111 & 142 & 142 & 165 & 191 & 19 \\
\hline SUMMER 1 & 266 & 200 & 281 & 390 & 338 & 381 & & 92 & 92 & 120 & 138 & 155 & 175 \\
\hline CRYSTAL RVR 3 & 89 & 139 & 184 & 184 & 184 & 184 & & 41 & 64 & 85 & 85 & 85 & 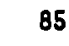 \\
\hline ARLEY 1 & 35 & 35 & 35 & 35 & 35 & 35 & & 16 & 16 & 16 & 16 & 16 & 1 \\
\hline RYR BEND 1 & 429 & 549 & 644 & 732 & 815 & 958 & & 78 & 166 & 117 & 133 & 149 & 173 \\
\hline IATERFORD 3 & 167 & 211 & 251 & 292 & 292 & 345 & & 71 & 96 & 107 & 125 & 125 & 14 \\
\hline HOPE CRK 1 & 528 & 698 & 698 & 850 & 1639 & 1639 & & 98 & 127 & 127 & 155 & 189 & 18 \\
\hline B日LEONTE 1 & 138 & 197 & 197 & 252 & 318 & 318 & & 63 & 96 & 98 & 115 & 146 & 145 \\
\hline SEABROOK-2 & 121 & 169 & 218 & 287 & 397 & 358 & & 51 & 72 & 92 & 113 & 136 & 152 \\
\hline 8日—GONTE 2 & 116 & 116 & 178 & 241 & 241 & 299 & & 53 & 53 & 80 & 110 & 110 & 136 \\
\hline COMANCHE PK $1 \mathrm{L2}$ & 268 & 368 & 392 & 463 & 538 & 654 & & 83 & 124 & 158 & 187 & 216 & 284 \\
\hline MCOUIRE 1 & 42 & 98 & 148 & 180 & 224 & 224 & & 18 & 41 & 83 & 76 & 95 & 95 \\
\hline NINE MILE PT2 & 96 & 384 & 394 & 454 & 842 & 842 & & $1 B$ & 55 & 55 & 81 & 115 & 115 \\
\hline CNLLATAY 1 & 37 & 111 & 177 & 177 & 226 & 283 & & 16 & 47 & 75 & 75 & 96 & 120 \\
\hline ICCUIRE 2 & 24 & 24 & 74 & 111 & 148 & 186 & & 10 & 10 & 31 & 47 & 62 & 79 \\
\hline DIABLO CNYH 2 & 16 & 18 & 57 & 86 & 138 & 172 & & 7 & 7 & 28 & 39 & 62 & 19 \\
\hline TRDJAN & 10 & 16 & 16 & 16 & 10 & 10 & & 5 & 5 & 5 & 5 & 5 & \\
\hline FARLEY 2 & & 6 & 5 & 5 & 5 & 5 & & & 2 & 2 & 2 & 2 & \\
\hline
\end{tabular}


TABLE A.6. Middle Case (with Extended Burnup) Maximum At-Reactor Capacity-Projected Cumulative Storage Requiremerits (contd)

ASSEMBLIES

MTIHM

POOL

B VALEY 2

DIABLO CMYN 1

CARRO $\perp$ COUNTY-1L2

GRAND GUF 2

GRAND QUF 1

WOLF CRK 1

SHOREAAM

$\overline{2015} \frac{2616}{2017} \frac{2618}{2619} \frac{2620}{117}: \overline{2615} \frac{2618}{13} \frac{2617}{13} \frac{2618}{30} \frac{2619}{56} \frac{262 \sigma}{54}$

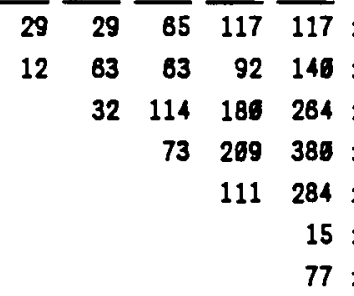

PWR TOTAL

\begin{tabular}{lllllll}
29486 & 32833 & 35414 & $: 12598$ & 14670 & \multicolumn{2}{c}{15203} \\
31313 & 34153 & 36940 & $:$ & 13462 & 14649 & 15863
\end{tabular}

BWR TOTAL

\begin{tabular}{llllllll}
41690 & 45585 & 48886 & $:$ & 7550 & \multicolumn{2}{c}{8253} & \multicolumn{2}{c}{8846} \\
43895 & 46927 & 50680 & $:$ & 7947 & 8496 & 9172
\end{tabular}

TOTAL

\begin{tabular}{ccccccc}
71176 & 78418 & 84288 & $: 20148$ & 22323 & \multicolumn{2}{c}{24848} \\
75268 & 81080 & 87620 & $:$ & 21349 & 23145 & 25635
\end{tabular}


TABLE A.7. Middle Case (with Extended Burnup) Maximum At-Reactor Capacity-Plus Transshipment--Projected Annual Storage Requirements

Assenblies

XTIHM

POOL

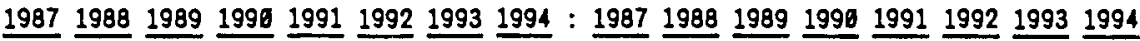

MILLSTONE 1

PALISADES

PRAIRIE ISL $1 \$ 2$

PILGRIM 1

BRUNSWICK 2

BRUNSWICK 1

CALVERT CLF 112

LACROSSE

FITZPATRICK

$28-\frac{124}{182} \frac{1}{195} \frac{112}{11}$ :

$\begin{array}{lllllll:}37 & 6 & 6 & 67 & 33 & 6 & 46:\end{array}$

33824 :

$1480:$

$780:$

$110:$

$84:$

$\frac{16}{22} \frac{10}{32} \frac{19}{34} \frac{19}{20}$

TOTAL PWR

TOTAL BWR.

$\begin{array}{lllllll}14 & 6 & 6 & 26 & 14 & 13 & 57\end{array}$

$28 \quad 124$

๑ $182 \quad 421277$ :

$\begin{array}{llllllll}5 & 6 & 22 & 0 & 32 & 6 & 75 & 50\end{array}$

TOTAL

$\begin{array}{lll}28 & 37 & 124\end{array}$

- $249 \quad 36$

459425 :

$\begin{array}{llllllll}5 & 14 & 22 & 6 & 58 & 14 & 88 & 107\end{array}$ 
TABLE A.7. Middle Case (with Extended Burnup) Maximum At-Reactor Capacity--

Plus Transshipment--Projected Annual Storage Requirements (contd)

POOL.

MILSTONE 1

PALISADES

PRAIRIE ISL $1 \$ 2$

PILGRIM 1

BRUNSWICK 2

BRUNSWICK 1

CALYERT CLF 112

LACROSSE

FITZPATRICX

INDIAN PT 3

SURRY 112

NORTH ANNA 112

COOPER STN

MILLSTONE 3

HADDAM NECK

MILLSTONE 2

FORT CALHOUN

DAYIS-BESSE 1

BRAIDNOOD 112

ZION 182

BYRON 112

BIG ROCK 1

POINT BEACH $1 \notin 2$

ENRICO FERUI2

OYSTER CRK 1

PEACHBOTTOM 3

PEACHBOTTOM 2

LIMERICK 1

\section{LIMERICK 2}

MAINE YANKEE

ARK NUCLEAR 2

ARK NUCLEAR 1

TURKEY PT $1 \$ 2$

ST LUCIE 1

ST LUCIE 2

COOK 112

SAN ONOFRE $1,2,23$

INDIAN PT 2

TASH NUCLEAR2

PERRY 112

DUANE ARMOLD

BROWNS FERRY1

BROWNS FERRY2

BROWNS FERRY3

$V$ YANKEE 1

KETAUNEE

B日LEONTE 2
Assenblies

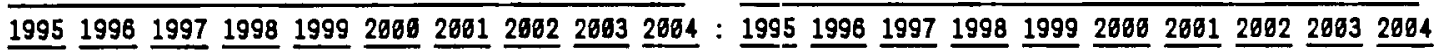

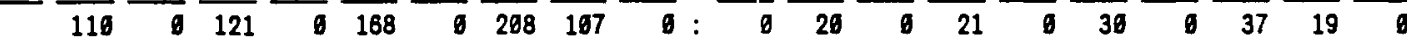

$\begin{array}{llllllllllllllllllll}64 & 34 & 1 & 45 & 45 & 34 & 66 & 0 & 53 & : & 25 & 13 & 6 & 16 & 18 & 9 & 13 & 26 & 0 & 21\end{array}$

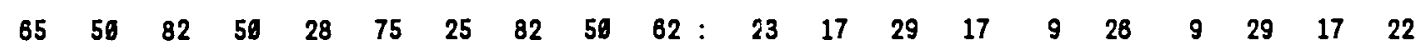

$\begin{array}{lllllllllllllllllllll}157 & 188 & 115 & 180 & 195 & 154: & 28 & 19 & 6 & 26 & 0 & 32 & 6 & 35 & 6 & 27\end{array}$

$\begin{array}{llllllllllllllllllll}148 & 191 & 168 & 127 & 0 & 93 & 183 & 0 & 141 & : & 28 & 19 & 6 & 29 & 24 & 6 & 17 & 34 & 6 & 26\end{array}$

$\begin{array}{lllllllllllllllllllll}148 & 184 & 167 & 168 & 93 & 9 & 98 & 141 & : & 28 & 9 & 34 & 26 & 9 & 31 & 17 & 0 & 18 & 28\end{array}$

$\begin{array}{llllllllllllllllllll}58 & 48 & 148 & 48 & 53 & 134 & 56 & 76 & 98 & 59: & 33 & 19 & 58 & 19 & 21 & 53 & 26 & 38 & 38 & 23\end{array}$

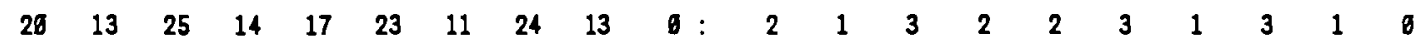

$\begin{array}{lllllllllllllllllllll}143 & 175 & 167 & 6 & 160 & 168 & 6 & 168 & 137: & 86 & 6 & 31 & 19 & \emptyset & 29 & 19 & 6 & 19 & 25\end{array}$

$\begin{array}{lllllllllllllllllllll}2 & 0 & 79 & 55 & 6 & 68 & 6 & 89 & 43 & 0 & : & 1 & 6 & 38 & 25 & 0 & 31 & 6 & 41 & 20 & 6\end{array}$

$\begin{array}{lllllllllllllllllllll}31 & 35 & 64 & 72 & 41 & 57 & 71 & 83 & 36 & 94 & : & 14 & 16 & 29 & 33 & 19 & 26 & 33 & 29 & 17 & 43\end{array}$

$\begin{array}{lllllllllllllllllllll}57 & 77 & 71 & 42 & 97 & 62 & 39 & 145 & 38 & 54: & 6 & 36 & 33 & 19 & 45 & 29 & 18 & 67 & 18 & 25\end{array}$

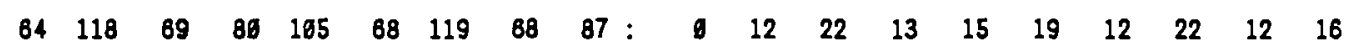

$\begin{array}{lllllllllllllllllll}14 & 51 & 52 & 37 & 65 & 37 & 6 & 39 & 43: & 6 & 6 & 24 & 24 & 17 & 39 & 17 & 6 & 18 & 26\end{array}$

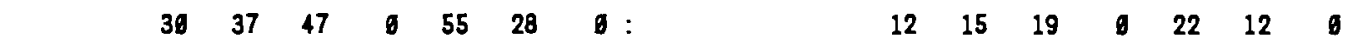

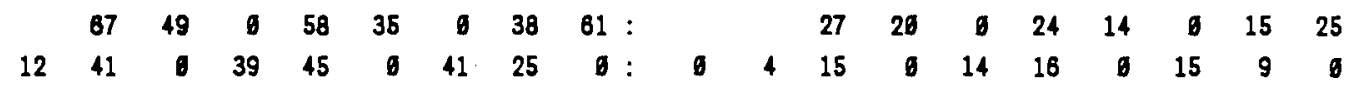

$\begin{array}{lllllllllllllllllll}25 & 49 & 48 & 6 & 37 & 71 & 0 & 53 & 0 & 0 & 12 & 0 & 19 & 22 & 0 & 17 & 33 & 6 & 25\end{array}$

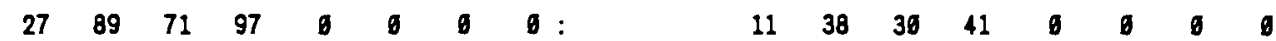

$\begin{array}{llllllllllllll}58 & 67 & 90 & 6 & 0 & 0 & 0 & 0 & 31 & 41 & 0 & 0 & 6 & 0\end{array}$

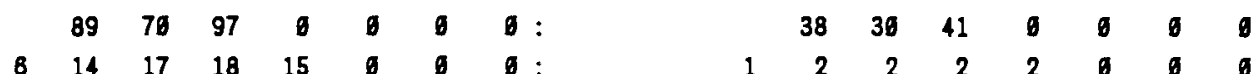

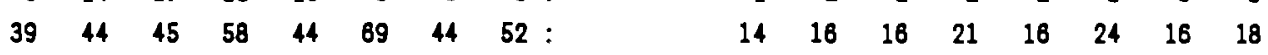

$224 \quad 249 \quad 271 \quad 158 \quad 0: \quad 41 \quad 9 \quad 45 \quad 0 \quad 49 \quad 29 \quad 6$

$\begin{array}{llllllllllllll}41 & 156 & 168 & 9 & 98 & 0 & : & 7 & 0 & 28 & 19 & 6 & 17 & 6\end{array}$

$\begin{array}{lllllllllllll}114 & 175 & 142 & 258 & 198 & 0 & 21 & 32 & 0 & 28 & 47 & 6 & 35\end{array}$

$\begin{array}{lllllllllll}220 & 143 & 145 & 188: & 41 & 26 & 6 & 27 & 35\end{array}$

$152 \quad 188 \quad 143 \quad 137 \quad 202:$

$\begin{array}{lllllll}272 & 244 & 155 & 193 & 0\end{array}$

$\begin{array}{lllllll}19 & 66 & 0 & 74 & 43 & 0\end{array}$

62675.58 :

$7154:$

$\begin{array}{lllll}45 & 66 & 53 & 33 & 82\end{array}$

$8152 \quad 6463$ :

5284663 :

$\begin{array}{lllll}91 & 48 & 91 & 164 & 65\end{array}$

$147116 \quad 11876$ :

$7 \quad 74$ b 57 :

$51 \quad 165 \quad 91 \quad 128$ :

$372 \quad 638$ :

44656 :

$78 \quad 223:$

158 :

1610 :

$435071:$

$2823 \quad 38$ :

21:

$58 \quad 44 \quad 28 \quad 35$

$\begin{array}{llllll}7 & 25 & 6 & 28 & 18 & 6\end{array}$

28 - $31 \quad 23$

$33 \quad 25$

$\begin{array}{lllll}21 & 36 & 24 & 15 & 38\end{array}$

$\begin{array}{lllll}25 & 19 & 0 & 20 & 23\end{array}$

$\begin{array}{llll}26 & 32 & 6 & 24\end{array}$

$\begin{array}{lllll}37 & 22 & 37 & 45 & 30\end{array}$

$55 \quad 50 \quad 50 \quad 30$

$3 \quad 34 \quad 28$

$\begin{array}{llll}9 & 29 & 18 & 23\end{array}$

$68 \quad 80$

8126

14040

296

$8 \quad 9 \quad 13$

$10 \quad 9 \quad 12$ 
TABLE A.7. Middle Case (with Extended Burnup) Maximum At-Reactor Capacity-Plus Transshipment--Projected Annual Storage Requirements (contd)

Assenblies

MT IHM

POOL

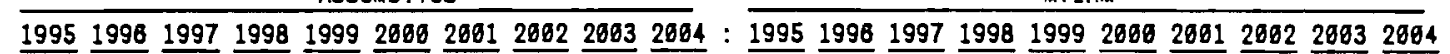

PUR TOTAL

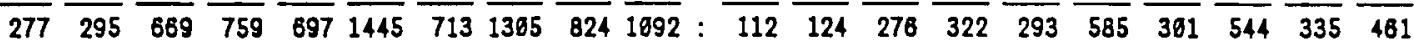

BNR TOTAL

$\begin{array}{llllllllll}616 & 394 & 568 & 1184 & 688 & 1877 & 973 & 2113 \quad 1648 \quad 2160 & \text { : }\end{array}$

$\begin{array}{lllll}111 & 70 \quad 91 \quad 213 & 124\end{array}$

$\begin{array}{lllll}338 & 176 & 381 & 298 & 383\end{array}$

TOTAL

$\begin{array}{lllllllllllllllllllll}893 & 689 & 1177 & 1943 & 1385 & 3322 & 1688 & 3418 & 2472 & 3192 & : & 223 & 194 & 367 & 535 & 417 & 923 & 477 & 928 & 633 & 944\end{array}$ 
TABLE A.7. Middle Case (with Extended Burnup) Maximum At-Reactor Capacity-Plus Transshipment--Projected Annual Storage Requirements (contd)

POOL

MILLSTONE 1

PALISADES

PRAIRIE ISL $1 \& 2$

PILGRIM 1

BRUNSWICK 2

BRUNSWICK 1

CALYERT CLF $1: 2$

FITZPATRICK

INDIAN PT 3

SURRY $1 \mathbf{1 2}$

NORTH ANNA 122

MILLSTONE 3

HADDAN NECK

MILLSTONE 2

FORT CALHOUN

DAVIS-BESSE 1

BRAIDWOOD $1 \mathbf{1 2}$

CARROLL COUNTY-1K2

ZION 1 \&2

BYRON 112

POINT BEACH $1 \mathbf{2 2}$

ENRICO FERMI2

OYSTER CRK 1

PEACHBOTTOM 3

PEACHBOTTOM 2

LIMERICK 1

LIMERICX 2

MAINE YANKEE

ARK NUCLEAR 2

ARK NUCLEAR 1

TURKEY PT $1 \mathbf{H 2}$

ST LUCIE 1

ST LUCIE 2

COOK 1 \&2

SAN ONOFRE $1,2,13$

INDIAN PT 2

WASH NUCLEAR2

PERRY $1 \mathbf{L 2}$

DUANE ARNOLD

BROWNS FERRY1

BROWNS FERRY2

BROWNS FERRY3
Assembl ies

NTIHM

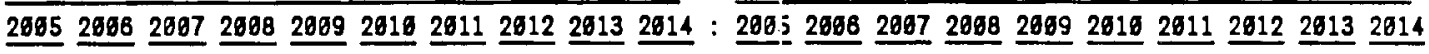

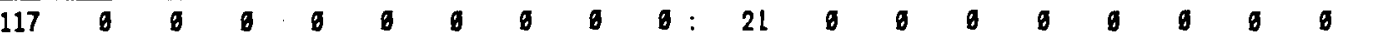

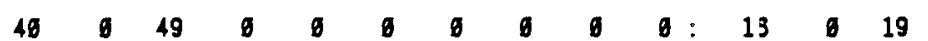

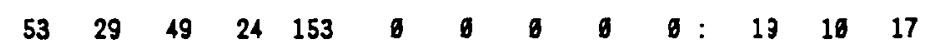

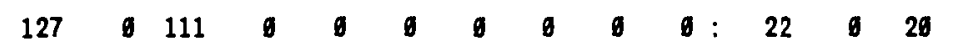

$\begin{array}{llllllllllllllll}117 & 104 & 91 & 6 & 89 & 177 & 1 & 129 & 127 & 22 & 6 & 19 & 17\end{array}$

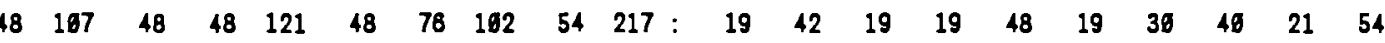

$\begin{array}{llllllllllllllllllll}126 & 195 & 0 & 145 & 104 & 0 & 142 & 127 & 125 & : & 0 & 23 & 19 & 0 & 26 & 19 & 0 & 26 & 23 & 22\end{array}$

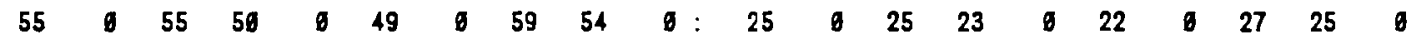

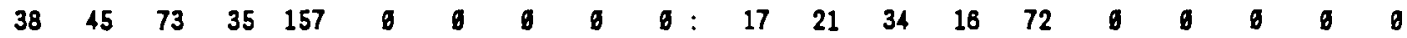

$\begin{array}{llllllllllllllllllll}49 & 41 & 76 & 58 & 38 & 144 & 73 & 62 & 107 & : & 42 & 23 & 19 & 35 & 27 & 18 & 66 & 34 & 29 & 49\end{array}$

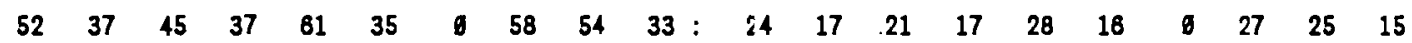

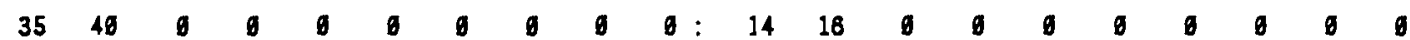

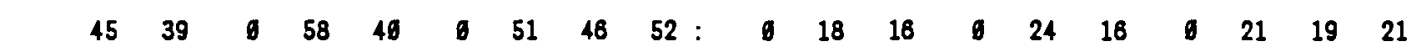

$\begin{array}{llllllllllllllllllllllll}38 & 40 & 0 & 30 & 0 & 0 & 0 & 0 & 0 & 0 & : & 14 & 14 & 0 & 11 & 0 & 0 & 0 & 0 & 0 & 0\end{array}$

$\begin{array}{lllllllllllllllllllll}44 & 0 & 39 & 37 & 9 & 36 & 69 & 0 & 59 & 49 & : & \vdots 1 & 0 & 18 & 17 & 9 & 17 & 32 & 0 & 23 & 23\end{array}$

$\begin{array}{llllllllllllllllllll}89 & 63 & 86 & 89 & 88 & 84 & 73 & 191 & 96 & 60: & : 8 & 27 & 34 & 25 & 36 & 27 & 31 & 43 & 41 & 25\end{array}$

$\begin{array}{llllllllllllllllllll}72 & 76 & 58 & 84 & 9 & 88 & 112 & 92 & 82 & : & 0 & 36 & 32 & 25 & 36 & 0 & 37 & 47 & 39 & 35\end{array}$

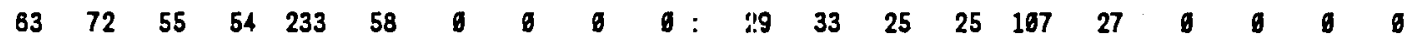

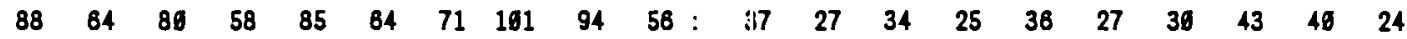

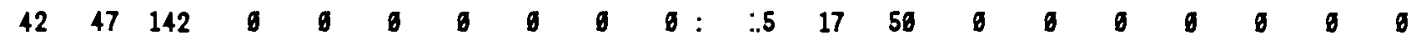

$\begin{array}{llllllllllllllllllll}224 & 0 & 264 & 0 & 224 & 147 & 0 & 259 & 0 & 152: & 11 & 0 & 37 & 0 & 41 & 27 & 0 & 47 & 6 & 28\end{array}$

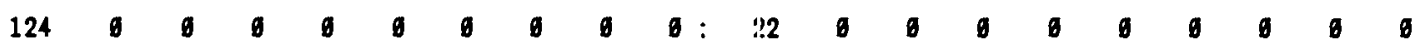

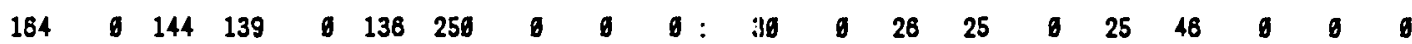

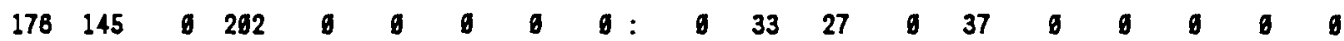

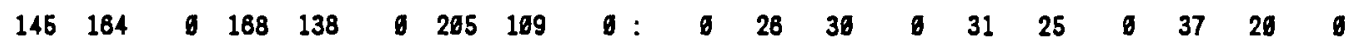

$\begin{array}{llllllllllllllllllll}176 & 154 & 207 & 219 & 0 & 291 & 219 & 0 & 186 & : & 32 & 28 & 6 & 38 & 49 & 9 & 53 & 49 & 6 & 33\end{array}$

$\begin{array}{llllllllllllllllllll}.46 & 52 & 0 & 0 & 0 & 0 & 0 & 0 & 0 & 0 & : & 17 & 20 & 0 & 0 & 0 & 0 & 0 & 0 & 0\end{array}$

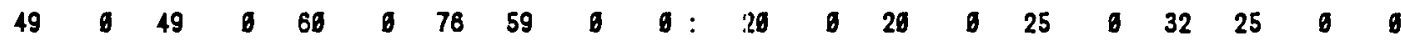

$\begin{array}{lllllllllllllllllllll}46 & 0 & 41 & 0 & 57 & 0 & 0 & 0 & 6 & 6 & : & 21 & 0 & 19 & 0 & 26 & 0 & 0 & 0 & 0 & 0\end{array}$

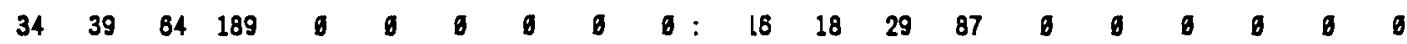

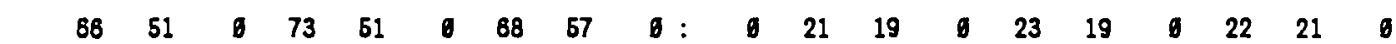

$\begin{array}{lllllllllllllllllllll}54 & 6 & 52 & 52 & 6 & 50 & 79 & 6 & 59 & 58 & : & 20 & 6 & 26 & 20 & 0 & 19 & 30 & 0 & 22 & 22\end{array}$

$\begin{array}{lllllllllllllllllllll}57 & 60 & 107 & 55 & 67 & 56 & 176 & 61 & 66 & 193 & : & 23 & 28 & 46 & 22 & 31 & 23 & 73 & 28 & 27 & 83\end{array}$

$\begin{array}{lllllllllllllllllllll}60 & 66 & 62 & 57 & 75 & 58 & 93 & 73 & 67 & 68 & : & 26 & 28 & 28 & 24 & 32 & 25 & 40 & 31 & 29 & 28\end{array}$

$\begin{array}{lllllllllllllllllllllll}48 & 0 & 42 & 41 & 0 & 0 & 0 & 0 & 6 & 0 & : & 22 & 0 & 19 & 19 & 0 & 0 & 0 & 0 & 0 & 0\end{array}$

$\begin{array}{lllllllllllllllllllll}165 & 117 & 94 & 91 & 134 & 88 & 163 & 128 & 116 & 115 & : & 18 & 21 & 17 & 16 & 24 & 15 & 29 & 23 & 20 & 20\end{array}$

$\begin{array}{lllllllllllllllllllll}415 & 6 & 379 & 288 & 0 & 301 & 493 & 0 & 471 & 358 & : & 72 & 0 & 69 & 52 & & 55 & 73 & 9 & 86 & 65\end{array}$

$\begin{array}{lllllllllllllllllllll}77 & 87 & 6 & 65 & 99 & 0 & 124 & 94 & 9 & 86 & : & 14 & 16 & 0 & 12 & 18 & 0 & 23 & 17 & 0 & 18\end{array}$

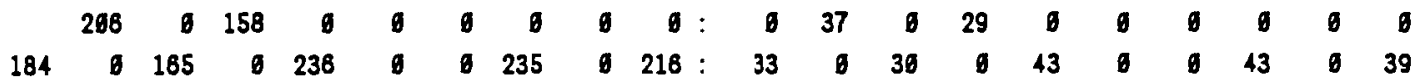

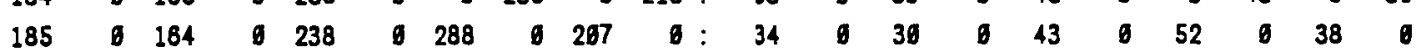


TABLE A.7. Middle Case (with Extended Burnup) Maximum At-Reactor Capacity-PIus Transshipment--Projected Annual Storage Requirements (contd)

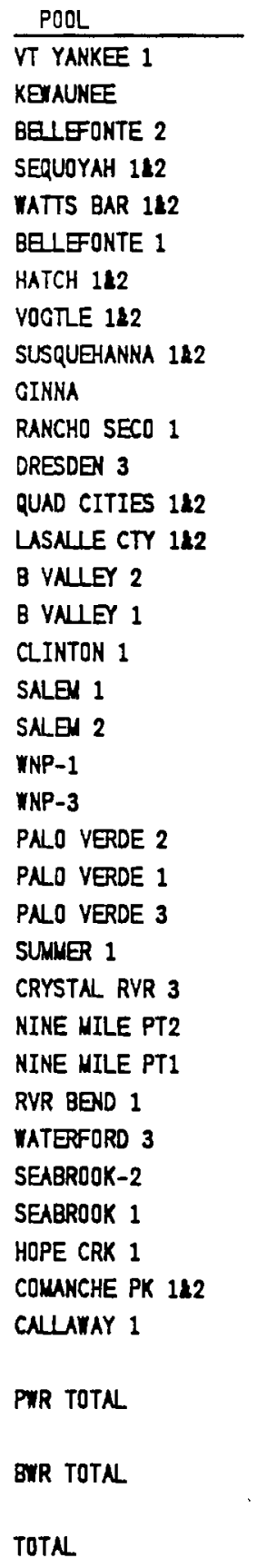

Asseablies

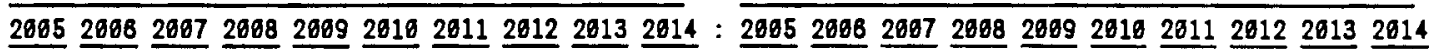

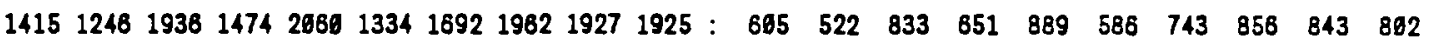

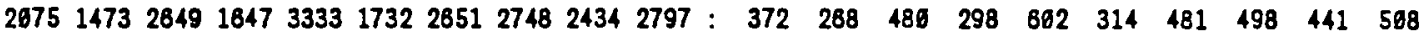
3490271945853121539338684343471043614722 : $977 \quad 796131394914919661224135412841311$ 
TABLE A.7. Middle Case (with Extended Burnup) Maximum At-Reactor Capacity-Plus Transshipment--Projected Annual Storage Requirements (contd)

\begin{tabular}{|c|c|c|c|c|c|c|c|c|c|c|c|c|c|}
\hline \multirow[b]{2}{*}{ POOL } & \multicolumn{6}{|c|}{ Assemblies } & \multicolumn{7}{|c|}{ MTIHM } \\
\hline & 2015 & 2618 & 2617 & $\underline{2618}$ & $\underline{2619}$ & 2620 & & 2015 & 2618 & 2617 & 2618 & 2619 & 2620 \\
\hline INDIAN PT 3 & & 82 & 6 & $\overline{6}$ & 6 & $\sqrt{6}$ & & 6 & 28 & 7 & 9 & 9 & 6 \\
\hline NORTH ANNA 112 & 55 & 63 & 157 & 6 & 6 & 6 & & 25 & 29 & 72 & 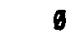 & g & 6 \\
\hline MILSTONE 3 & 43 & 48 & 38 & 6 & 34 & 57 & & 26 & 18 & 18 & 8 & 16 & 26 \\
\hline DAVIS-BESSE 1 & & 68 & 58 & 6 & 6 & 6 & & 6 & 28 & 26 & - & g & 0 \\
\hline BRAIDWOOD 112 & 85 & 89 & 72 & 84 & 86 & 164 & : & 36 & 38 & 30 & 27 & 25 & 44 \\
\hline CARROLL COUNTY-112 & 70 & 78 & 82 & 82 & 66 & 84 & & 30 & 33 & 35 & 35 & 28 & 38 \\
\hline BYRON 122 & 85 & 89 & 71 & 59 & 62 & 162 & & 36 & 38 & 30 & 25 & 28 & 43 \\
\hline ENRICO FERMI2 & & 233 & 150 & 6 & 155 & 6 & & 6 & 42 & 27 & 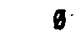 & 28 & $\theta$ \\
\hline LIMERICX 1 & 168 & 135 & 6 & 125 & 154 & 6 & & 31 & 25 & 6 & 23 & 28 & 8 \\
\hline LIMERICX 2 & 213 & 6 & 267 & 219 & 6 & 199 & : & 39 & 6 & 38 & 48 & 6 & 36 \\
\hline ST LUCIE 2 & & 71 & 68 & 6 & 48 & 56 & : & 6 & 27 & 25 & 6 & 18 & 21 \\
\hline SAN ONOFRE $1,2,13$ & 57 & 128 & 6 & 131 & 6 & 141 & : & 24 & 51 & 6 & 58 & 6 & 68 \\
\hline WASH NUCLEAR2 & 85 & 141 & 133 & 106 & 92 & 110 & : & 15 & 25 & 23 & 18 & 16 & 19 \\
\hline PERRY 112 & & 419 & 381 & 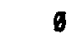 & 319 & 452 & : & 0 & 76 & 69 & 6 & 58 & 82 \\
\hline BROWNS FERRY3 & 145 & 6 & 234 & $\theta$ & $\theta$ & $\theta$ & : & 28 & 6 & 42 & 6 & 0 & 8 \\
\hline B日LEONTE 2 & 67 & 6 & 68 & 85 & g & 58 & : & 31 & 6 & 27 & 36 & 6 & 26 \\
\hline SERUOYAH 112 & 42 & 138 & 45 & 51 & 90 & ø & : & 15 & 63 & 21 & 23 & 41 & 6 \\
\hline WATTS BAR 112 & 61 & 112 & 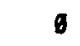 & 99 & 44 & 69 & : & $2 \varepsilon_{1}$ & 51 & 6 & 45 & 26 & 32 \\
\hline 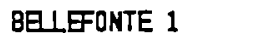 & 73 & 59 & g & 55 & 66 & $\theta$ & : & $3: i$ & 27 & 6 & 25 & 36 & 8 \\
\hline VDGTLE $1 \$ 2$ & 89 & 80 & B4 & 84 & 91 & 79 & : & 41. & 37 & 30 & 39 & 42 & 36 \\
\hline SUSqUEGANKA $1 \mathbf{2}$ & 124 & 421 & 194 & 150 & 272 & 169 & : & $2 ! !$ & 74 & 34 & 26 & 48 & 36 \\
\hline LASALLE CTY 112 & 234 & 195 & 184 & 283 & 124 & 190 & : & $4 i$ & 36 & 34 & 52 & 23 & 35 \\
\hline B VAШEY 2 & 56 & 46 & $\theta$ & 36 & 52 & 0 & : & 211 & 21 & 6 & 17 & 24 & 8 \\
\hline CLINTON 1 & & 159 & 112 & 0 & 116 & 130 & : & II & 29 & 29 & 6 & 21 & 24 \\
\hline SALEN 1 & & 88 & 84 & 6 & 6 & 0 & : & $\|$ & 31 & 29 & 6 & 6 & 8 \\
\hline SALEN 2 & 43 & 67 & 6 & 56 & 56 & 0 & : & 211 & 31 & 6 & 23 & 26 & 8 \\
\hline MNP-1 & 41 & 48 & 49 & 49 & 46 & 51 & : & 13 & 22 & 22 & 22 & 18 & 23 \\
\hline WNP-3 & 43 & 50 & 51 & 51 & 42 & 53 & : & 17 & 22 & 22 & 22 & 18 & 23 \\
\hline PNO VERDE 2 & 47 & 50 & 40 & 38 & 36 & 58 & : & 21 & 22 & 17 & 17 & 16 & 25 \\
\hline PALD VERDE 1 & 49 & 51 & 41 & 36 & 36 & 58 & : & 21 & 22 & 18 & 16 & 16 & 25 \\
\hline PALO VERDE 3 & 44 & 40 & 34 & 42 & 46 & 39 & : & 19 & 17 & 15 & 18 & 26 & 17 \\
\hline SUMMER 1 & 25 & g & 61 & 39 & 38 & 43 & : & 11 & 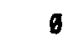 & 28 & 18 & 17 & 20 \\
\hline CRYSTAL RVR 3 & & 50 & 45 & 0 & $\theta$ & 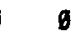 & : & B & 23 & 21 & 6 & 6 & $\theta$ \\
\hline MINE MILE PT2 & & 214 & $\theta$ & 150 & 188 & $\theta$ & : & $\theta$ & 38 & 6 & 27 & 34 & 6 \\
\hline RVR BEND 1 & 112 & 120 & 95 & 88 & 83 & 143 & : & 28 & 22 & 17 & 16 & 15 & 25 \\
\hline WATERFORD 3 & 43 & 44 & 40 & 41 & $\theta$ & 53 & : & 18 & 19 & 17 & 18 & 6 & 23 \\
\hline SEABROOK-2 & 41 & 48 & 49 & 49 & 40 & 51 & : & 17 & 20 & 21 & 21 & 17 & 22 \\
\hline SEABROOK 1 & 48 & 52 & 41 & 30 & 34 & 56 & : & 20 & 22 & 17 & 13 & 14 & 24 \\
\hline YANKEE-RONE 1 & 6 & 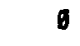 & g & - & $\theta$ & 9 & : & g & 6 & 6 & 6 & 6 & 8 \\
\hline HOPE CRK 1 & 263 & 168 & 0 & 154 & 189 & 6 & & 37 & 31 & 6 & 28 & 34 & $\theta$ \\
\hline COLANCHE PK 112 & 9 & 162 & 84 & 71 & 73 & 118 & & 10 & 41 & 34 & 29 & 29 & 48 \\
\hline CALLAIAY 1 & & 74 & 66 & ø & 49 & 57 & & 6 & 31 & 28 & 6 & 21 & 24 \\
\hline
\end{tabular}


TABLE A.7. Middle Case (with Extended Burnup) Maximum At-Reactor Capacity-Plus Transshipment--Projected Annual Storage Requirements (contd)

Assemblies

POOL

TROJAN

FARLEY 2

DIAQLO CNYN 1

DIABLO CNYN 2

HARRIS 1

GRAND GUF 2

GRAND GUF 1

YOLF CRK 1

SHOREYAM

PUR TOTAL

BUR TOTAL

TOTAL

\begin{tabular}{|c|c|c|c|c|c|c|c|c|c|c|c|c|c|}
\hline \multicolumn{7}{|c|}{ 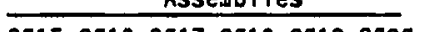 } & \\
\hline 2015 & 2016 & 2017 & 201 & & 2019 & 2020 & : & 2015 & 2016 & $\underline{2617}$ & 2018 & $\underline{2619}$ & 2020 \\
\hline \multirow[t]{10}{*}{10} & $\theta$ & $\theta$ & & $\theta$ & $g$ & 6 & : & 5 & 6 & $\theta$ & $\theta$ & g & $\theta$ \\
\hline & 40 & 0 & & D & g & g & : & (6) & 18 & $\theta$ & 6 & 0 & g \\
\hline & 28 & 51 & & 6 & 29 & 48 & : & $\theta$ & 13 & 23 & 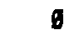 & 13 & 22 \\
\hline & & 41 & & 9 & 50 & 36 & : & & & 19 & 13 & 23 & 17 \\
\hline & & & 1 & 9 & 38 & 6 & : & & & & 9 & 17 & g \\
\hline & & & 3 & 3 & 136 & 171 & : & & & & 8 & 25 & 31 \\
\hline & & & & & 151 & 173 & : & & & & & 27 & 30 \\
\hline & & & & & & 15 & : & & & & & & 7 \\
\hline & & & & & & 77 & $:$ & & & & & & 14 \\
\hline & & & & & & & : & & & & & & \\
\hline \multirow[t]{2}{*}{1318} & 1919 & 1468 & 127 & & 1218 & 1486 & : & 579 & 845 & 648 & 559 & 537 & 644 \\
\hline & & & & & & & $:$ & & & & & & \\
\hline \multirow[t]{2}{*}{1284} & 2265 & 1696 & 130 & & 1979 & 1814 & : & 233 & 398 & 398 & 235 & 357 & 327 \\
\hline & & & & & & & $:$ & & & & & & \\
\hline 2800 & 4124 & 3158 & 257 & & 3197 & 3306 & : & 812 & 1243 & 951 & 794 & 894 & 976 \\
\hline
\end{tabular}

A. 61 
TABLE A.8. Middle Case (with Extended Burnup) Maximum At-Reactor Capacity-Plus Transshipment--Projected Cumulative Storage Requirements

Assemblies

VTIHM

\begin{tabular}{|c|c|c|c|c|c|c|c|c|c|c|c|c|c|c|c|c|}
\hline & & & & & & & & & & & & & & & & \\
\hline POOL & $\underline{1987}$ & $\underline{1988}$ & $\underline{1989}$ & $\underline{1998}$ & 1991 & $\underline{1992}$ & 1993 & $\underline{1994}$ & $: \underline{1987}$ & $\underline{1388}$ & $\underline{1989}$ & $\underline{1990}$ & $\underline{1991}$ & $\underline{1992}$ & $\underline{1993}$ & 1994 \\
\hline MIULSTONE 1 & 26 & 26 & 150 & 158 & 332 & 332 & 527 & 639 & 5 & 5 & 27 & 27 & 59 & 59 & 94 & 114 \\
\hline PNISAOES & & 37 & 37 & 37 & 184 & 137 & 137 & 177 & : & 14 & 14 & 14 & 41 & 53 & 53 & 69 \\
\hline PRAIRIE ISL $1 \$ 2$ & & & & & & 3 & 41 & 85 & $:$ & & & & & 1 & 14 & 22 \\
\hline PILGRIX 1 & & & & & & & 148 & 148 & : & & & & & & 28 & 28 \\
\hline BRUNSWICX 2 & & & & & & & 78 & 78 & : & & & & & & 15 & 15 \\
\hline BRUNSIIICK 1 & & & & & & & & 110 & : & & & & & & & 21 \\
\hline CALYERT CLF 112 & & & & & & & & 84 & $:$ & & & & & & & 33 \\
\hline LACROSSE & & & & & & & & B & : & & & & & & & 1 \\
\hline FITZPATRICK & & & & & & & & 49 & : & & & & & & & 9 \\
\hline PWR TOTAL & & 37 & 37 & 37 & 104 & 140 & 178 & 326 & : & 14 & 14 & 14 & 41 & 54 & 67 & 124 \\
\hline BWR TOTAL & 28 & 28 & 158 & 150 & 332 & 332 & 753 & 1030 & 5 & 5 & 27 & 27 & 59 & 59 & 134 & 184 \\
\hline TOTAL & 28 & 83 & 187 & 187 & 436 & 472 & 931 & 1356 & 5 & 19 & 41 & 41 & 160 & 114 & 201 & 389 \\
\hline
\end{tabular}


TABLE A.8. Middle Case (with Extended Burnup) Maximum At-Reactor Capacity-Plus Transshipment--Projected Cumulative Storage Requirements (contd)

\begin{tabular}{|c|c|c|c|c|c|c|c|c|c|c|c|c|c|c|c|c|c|c|c|c|c|}
\hline \multirow[b]{2}{*}{ POOL } & \multicolumn{10}{|c|}{ Assenblies } & & \multicolumn{10}{|c|}{ MTIHM } \\
\hline & 1995 & $\underline{1996}$. & 1997 & 1998 & $\underline{1999}$ & $\underline{2000}$ & $\underline{2601}$ & $\underline{2002}$ & 2093 & $\underline{2904}$ & & $\underline{1995}$ & $\underline{1996}$ & 1997 & $\underline{1998}$ & $\underline{1999}$ & $\underline{2960}$ & 2801 & 2982 & 2003 & $\underline{2804}$ \\
\hline MILLSTONE 1 & 639 & 749 & 749 & $87 \theta$ & 870 & 1638 & 1038 & 1246 & 1353 & 1353 & & 114 & 133 & 133 & 155 & 155 & 184 & 184 & 221 & 240 & 240 \\
\hline PALISADES & 241 & 275 & 275 & 316 & 361 & 361 & 395 & 461 & 461 & 514 & $:$ & 94 & 167 & 107 & 123 & 141 & 141 & 154 & 179 & 179 & 260 \\
\hline PRAIRIE ISL 112 & 136 & 180 & 262 & 312 & 340 & 415 & 440 & 522 & 572 & 634 & : & 45 & 63 & 91 & 169 & 118 & 144 & 153 & 182 & 200 & 221 \\
\hline PILGRIM 1 & 305 & 411 & 411 & 526 & 528 & 768 & 766 & 961 & 981 & 1055 & & 54 & 73 & 73 & 93 & 93 & 125 & 125 & 160 & 160 & 187 \\
\hline BRL'NSWICK 2 & 226 & 327 & 327 & 433 & 580 & 560 & 653 & 836 & 836 & 977 & 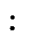 & 42 & 61 & 61 & 81 & 165 & 165 & 122 & 156 & 156 & 183 \\
\hline BRUNSWICK 1 & 258 & 258 & 442 & 549 & 549 & 715 & 868 & 868 & 906 & 1047 & : & 48 & 48 & 83 & 163 & 163 & 134 & 151 & 151 & 169 & 196 \\
\hline CALYERT CLF $1 \$ 2$ & 142 & 190 & 338 & 386 & 439 & 573 & 623 & 699 & 797 & 856 & . & 56 & 75 & 133 & 152 & 172 & 225 & 245 & 275 & 313 & 336 \\
\hline LACROSSE & 28 & 39 & 64 & 78 & 95 & 118 & 129 & 153 & 166 & 166 & & 3 & 4 & 7 & 8 & 16 & 13 & 14 & 17 & 18 & 18 \\
\hline FITZPATRICK & 192 & 192 & 367 & 474 & 474 & 634 & 742 & 742 & 848 & 985 & & 35 & 35 & 66 & 85 & 85 & 114 & 133 & 133 & 152 & 177 \\
\hline INDIAN PT 3 & 2 & 2 & 81 & 136 & 136 & 264 & 284 & 293 & 336 & 336 & 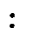 & 1 & 1 & 37 & 62 & 62 & 93 & 93 & 134 & 153 & 153 \\
\hline SURRY 112 & 31 & 66 & 130 & 202 & 243 & 300 & 371 & 434 & 476 & 564 & : & 14 & 30 & 60 & 93 & 112 & 138 & 176 & 199 & 216 & 259 \\
\hline NORTH ANNA $1 \mathbf{1 2}$ & 57 & 134 & 285 & 247 & 344 & 468 & 445 & 590 & 628 & 882 & 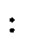 & 28 & 62 & 95 & 114 & 159 & 187 & 265 & 272 & 290 & 315 \\
\hline COOPER STN & & 64 & 182 & 251 & 331 & 436 & 504 & 623 & 891 & 778 & 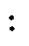 & 6 & 12 & 33 & 46 & 68 & 79 & 92 & 114 & 128 & 142 \\
\hline VIUSTONE 3 & & 14 & 65 & 117 & 154 & 219 & 256 & 256 & 295 & 338 & : & b & 6 & 30 & 54 & 71 & 161 & 118 & 118 & 136 & 156 \\
\hline HADDAM NECK & & & & 30 & 67 & 114 & 114 & 169 & 197 & 197 & 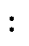 & & & & 12 & 27 & 47 & 47 & 69 & 81 & 81 \\
\hline MILLSTONE 2 & & & 67 & 116 & 116 & 174 & 209 & 209 & 247 & 368 & : & & & 27 & 47 & 47 & 71 & 85 & 85 & 106 & 125 \\
\hline =ORT CALHOUN & & 12 & 53 & 53 & 92 & 137 & 137 & 178 & 263 & 263 & : & $\theta$ & 4 & 19 & 19 & 33 & 49 & 49 & 64 & 73 & 73 \\
\hline DAVIS-BESSE 1 & & 25 & 25 & 65 & 113 & 113 & 150 & 221 & 221 & 274 & $:$ & $\theta$ & 12 & 12 & 30 & 53 & 53 & 70 & 103 & 163 & 128 \\
\hline BRAIDWOOD 142 & & & 27 & 116 & 187 & 284 & 284 & 284 & 284 & 284 & : & & & 11 & 49 & 79 & $12 \theta$ & 120 & 120 & 126 & 126 \\
\hline ZION 112 & & & & 58 & 125 & 215 & 215 & 215 & 215 & 215 & ${ }^{\circ}$ & & & & 27 & 57 & 98 & 98 & 98 & 98 & 98 \\
\hline BYRON 112 & & & & 89 & 159 & 256 & 258 & 256 & 256 & 256 & 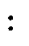 & & & & 38 & 67 & 168 & 168 & 168 & 168 & 168 \\
\hline BIG ROCK 1 & & & 6 & 28 & 37 & 55 & 76 & 78 & 76 & 76 & : & & & 1 & 3 & 5 & 7 & 9 & 9 & 9 & 9 \\
\hline POINT BEACH 112 & & & 39 & 83 & 128 & 188 & 236 & 299 & 343 & 395 & : & & & 14 & 29 & 45 & 86 & 82 & 106 & 122 & 140 \\
\hline ENRICO FERMI2 & & & & 224 & 224 & 473 & 473 & 744 & 962 & 962 & $:$ & & & & 41 & 41 & 86 & 86 & 135 & 164 & 164 \\
\hline OYSTER CRK 1 & & & & 41 & 41 & 197 & 303 & 303 & 401 & 461 & : & & & & 7 & 7 & 35 & 54 & 54 & 71 & 71 \\
\hline PEACHBOTTOM 3 & & & & 114 & 289 & 289 & 431 & 889 & 689 & 879 & : & & & & 21 & 53 & 53 & 79 & 128 & 128 & 160 \\
\hline PEACHBOTTOX 2 & & & & & & 220 & 363 & 363 & 568 & 698 & : & & & & & & 41 & 67 & 87 & 94 & 129 \\
\hline LIMERICK 1 & & & & 152 & 152 & 348 & 483 & 483 & 626 & 822 & ${ }^{\circ}$ & & & & 28 & 28 & 62 & 88 & 88 & 113 & 150 \\
\hline LIMERICX 2 & & & & & 272 & 516 & 516 & 871 & 864 & 864 & $:$ & & & & & 50 & 94 & 94 & 122 & 157 & 157 \\
\hline MAINE YANKEE & & & & & 19 & 85 & 85 & 159 & 262 & 262 & : & & & & & 7 & 32 & 32 & 68 & 78 & 76 \\
\hline ARK NUCLEAR 2 & & & & & & 62 & 62 & 137 & 137 & 193 & 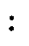 & & & & & & 28 & 28 & 57 & 57 & 86 \\
\hline ARK NUCLEAR 1 & & & & & & & & 71 & 71 & 125 & & & & & & & & & 33 & 33 & 58 \\
\hline TURKEY PT $1: 2$ & & & & & & 45 & 111 & 184 & 197 & 279 & & & & & & & 21 & 51 & 75 & 90 & 128 \\
\hline ST LUCIE 1 & & & & & & 81 & 133 & 133 & 197 & 260 & & & & & & & 25 & 45 & 45 & 65 & 88 \\
\hline ST LUCIE 2 & & & & & & & 52 & 136 & 136 & 199 & & & & & & & & 20 & 52 & 52 & 75 \\
\hline $\operatorname{coOK} 112$ & & & & & & 91 & 139 & 230 & 334 & 399 & 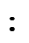 & & & & & & 37 & 59 & 96 & 140 & 176 \\
\hline SAN ONOFRE $1,2,13$ & & & & & & 147 & 283 & 263 & 381 & 451 & & & & & & & 55 & 164 & 164 & 154 & 184 \\
\hline INDIAN PT 2 & & & & & & & 7 & 81 & 81 & 138 & & & & & & & & 3 & 37 & 37 & 63 \\
\hline WASH MUCLEAR2 & & & & & & & 51 & 216 & 307 & 435 & & & & & & & & 9 & 38 & 54 & 77 \\
\hline PERRY $1 \not 2$ & & & & & & & & 372 & 372 & 810 & & & & & & & & & 68 & 68 & 148 \\
\hline DUANE ARNOLD & & & & & & & & 44 & 109 & 109 & & & & & & & & & 8 & 20 & 20 \\
\hline BROWNS FERRY1 & & & & & & & & 76 & 76 & 299 & & & & & & & & & 14 & 14 & 54 \\
\hline BROWNS FERRY2 & & & & & & & & & 158 & 158 & & & & & & & & & & 29 & 29 \\
\hline BROWNS FERRY3 & & & & & & & & & 181 & 161 & & & & & & & & & & 29 & 29 \\
\hline
\end{tabular}


TABLE A.8. Middle Case (with Extended Burnup) Maximum At-Reactor Capacity-Plus Transshipment--Projected Cumulative Storage Requirements (contd)

Assenblies

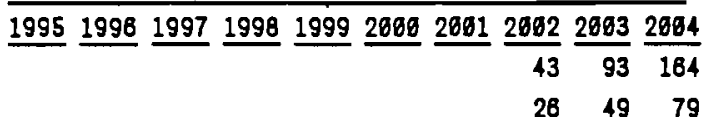
VT YANKEE 1 KEMAUNEE BELLEONTE 2

PWR TOTAL

BWR TOTAL

TOTAL

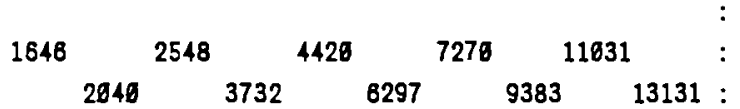

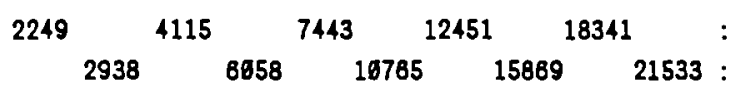

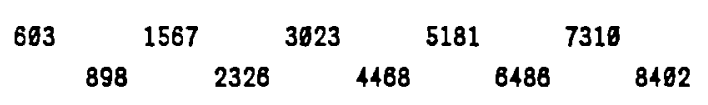

NTIHM

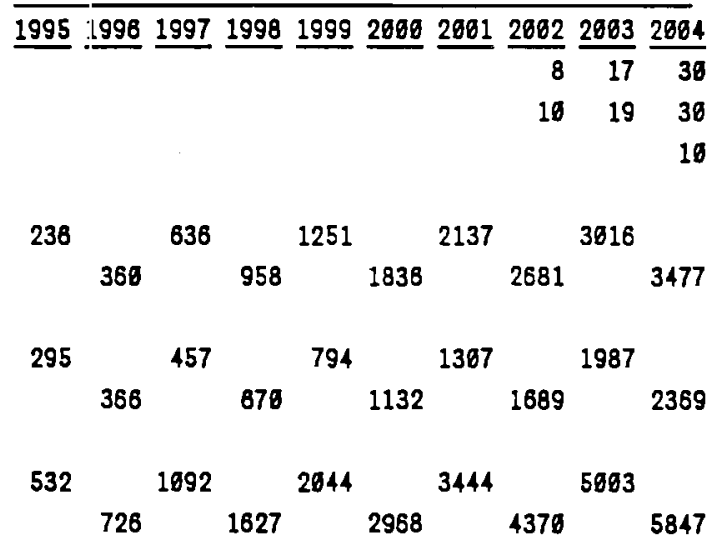


TABLE A.8. Middle Case (with Extended Burnup) Maximum At-Reactor Capacity-Plus Transshipment--Projected Cumulative Storage Requirements

(contd)

Assenblies

МTIHM

POOL

MIUSTONE 1

PALISADES

PRAIRIE ISL 122

PILGRIM 1

BRUNSWICK 2

BRUNSWICK 1

CALYERT CLF 1 L2

LACROSSE

FITZPATRICK

INDIAN PT 3

SURRY 112

NORTH ANNA 112

COOPER STN

MILLSTONE 3

HADOAM NECK

MILLSTONE 2

FORT CALHOUN

DAVIS-BESSE 1

BRAIDYOOD $1 \mathrm{L2}$

CARROL COUNTY-112

ZION 112

BYRON 112

BIG ROCK 1

POINT BEACH $1 \$ 2$

ENRICO FERMI2

OYSTER CRK 1

PEACHBOTTOM 3

PEACHBOTTOM 2

LIMERICK 1

LIMERICK 2

MAINE YANKEE

ARK NUCLEAR 2

ARK NUCLEAR 1

TURKEY PT 112

ST LUCIE 1

ST LUCIE 2

COOK 112

SAN ONOFRE $1,2,23$

INDIAN PT 2

MASH NUCLEAR2

PERRY $1 \angle 2$

DUANE ARNOLD

BROWNS FERRY1

BROWNS FERRY2

BROWNS FERRY3

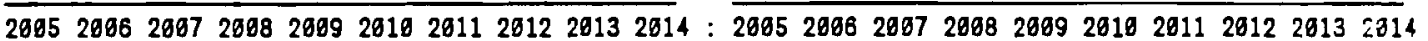

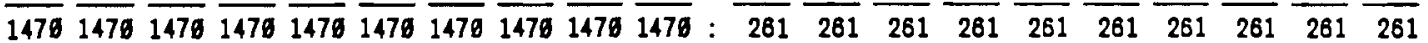
$\begin{array}{lllllllllllllllllllll}554 & 554 & 683 & 663 & 683 & 683 & 663 & 663 & 693 & 683 & : & 216 & 216 & 235 & 235 & 235 & 235 & 235 & 235 & 235 & 235\end{array}$ $\begin{array}{lllllllllllllllllllll}687 & 716 & 765 & 789 & 942 & 942 & 942 & 942 & 942 & 942 & : & 246 & 249 & 286 & 275 & 338 & 330 & 336 & 330 & 330 & 330\end{array}$ $\begin{array}{lllllllllllllllllllll}1182 & 1182 & 1293 & 1293 & 1293 & 1293 & 1293 & 1293 & 1293 & 1293 & : & 269 & 269 & 229 & 229 & 229 & 229 & 229 & 229 & 229 & 229\end{array}$

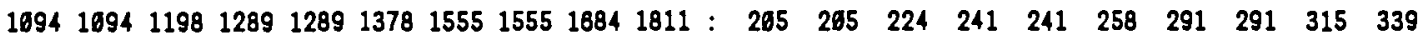

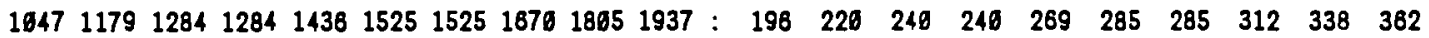
$\begin{array}{lllllllllllllllllllll}964 & 1611 & 1959 & 1167 & 1228 & 1276 & 1352 & 1454 & 1508 & 1725 & : & 355 & 397 & 418 & 435 & 482 & 501 & 531 & 571 & 592 & 647\end{array}$ $\begin{array}{lllllllllllllllllllll}168 & 168 & 168 & 166 & 166 & 166 & 168 & 168 & 166 & 166 & : & 18 & 18 & 18 & 18 & 18 & 18 & 18 & 18 & 18 & 18\end{array}$ $\begin{array}{lllllllllllllllllllll}85 & 1111 & 1216 & 1216 & 1361 & 1465 & 1465 & 1667 & 1734 & 1859 & : & 177 & 206 & 219 & 219 & 245 & 263 & 263 & 289 & 312 & 334\end{array}$ $\begin{array}{lllllllllllllllllllll}391 & 391 & 446 & 496 & 496 & 545 & 545 & 684 & 658 & 658 & : & 178 & 178 & 203 & 226 & 226 & 249 & 249 & 275 & 398 & 380\end{array}$ $\begin{array}{lllllllllllllllllllll}662 & 647 & 726 & 755 & 912 & 912 & 912 & 912 & 912 & 912 & : & 276 & 297 & 336 & 347 & 419 & 419 & 419 & 419 & 419 & 419\end{array}$ $\begin{array}{lllllllllll:llllllllll}772 & 821 & 862 & 938 & 996 & 1034 & 1178 & 1251 & 1313 & 1429 & : & 356 & 379 & 398 & 433 & 460 & 477 & 544 & 577 & 606 & 655\end{array}$ $\begin{array}{lllllllllllllllllllll}778 & 778 & 778 & 778 & 778 & 778 & 778 & 778 & 778 & 778 & 142 & 142 & 142 & 142 & 142 & 142 & 142 & 142 & 142 & 142\end{array}$ $\begin{array}{lllllllllllllllllllll}396 & 427 & 472 & 599 & 570 & 695 & 685 & 663 & 717 & 750 & : & 186 & 197 & 218 & 235 & 263 & 279 & 279 & 386 & 331 & 346\end{array}$ $\begin{array}{lllllllllllllllllllll}232 & 272 & 272 & 272 & 272 & 272 & 272 & 272 & 272 & 272 & \text { : } & 95 & 112 & 112 & 112 & 112 & 112 & 112 & 112 & 112 & 112\end{array}$ $\begin{array}{lllllllllllllllllllll}368 & 353 & 392 & 392 & 456 & 498 & 496 & 541 & 587 & 639 & : & 125 & 143 & 159 & 159 & 183 & 199 & 199 & 220 & 238 & 259\end{array}$ $\begin{array}{lllllllllllllllllllll}241 & 281 & 281 & 311 & 311 & 311 & 311 & 311 & 311 & 311 & : & 87 & 101 & 161 & 112 & 112 & 112 & 112 & 112 & 112 & 112\end{array}$ $\begin{array}{lllllllllllllllllllll}318 & 318 & 357 & 394 & 394 & 438 & 499 & 499 & 549 & 598 & : & 149 & 149 & 167 & 184 & 184 & 261 & 234 & 234 & 257 & 280\end{array}$ $\begin{array}{lllllllllllllllllllll}373 & 436 & 516 & 576 & 662 & 726 & 799 & 966 & 998 & 1658 & : & 158 & 184 & 218 & 244 & 286 & 367 & 338 & 381 & 421 & 446\end{array}$ $\begin{array}{llllllllllllllllllll}72 & 148 & 296 & 298 & 296 & 378 & 496 & 682 & 684 & : & 6 & 38 & 63 & 87 & 123 & 123 & 169 & 297 & 246 & 281\end{array}$ $\begin{array}{lllllllllllllllllllll}278 & 350 & 496 & 469 & 692 & 750 & 756 & 756 & 75 \theta & 75 \theta: & 127 & 16 \theta & 186 & 216 & 316 & 343 & 343 & 343 & 343 & 343\end{array}$ $\begin{array}{lllllllllllllllllllll}344 & 468 & 488 & 546 & 631 & 895 & 766 & 867 & 961 & 1617 & : & 145 & 173 & 266 & 231 & 267 & 294 & 324 & 367 & 496 & 430\end{array}$

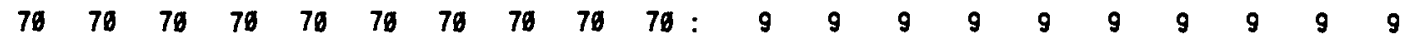
$\begin{array}{lllllllllllllllllllll}437 & 484 & 626 & 626 & 626 & 626 & 626 & 626 & 628 & 626 & : & 155 & 172 & 222 & 222 & 222 & 222 & 222 & 222 & 222 & 222\end{array}$ $\begin{array}{lllllllllllllllllllll}1126 & 1126 & 1338 & 1336 & 1554 & 1761 & 1761 & 1960 & 1960 & 2112 & \text { : } & 265 & 265 & 242 & 242 & 283 & 310 & 310 & 357 & 357 & 384\end{array}$ $\begin{array}{lllllllllllllllllllll}525 & 525 & 525 & 525 & 625 & 525 & 525 & 525 & 525 & 625 & : & 93 & 93 & 93 & 93 & 93 & 93 & 93 & 93 & 93 & 93\end{array}$

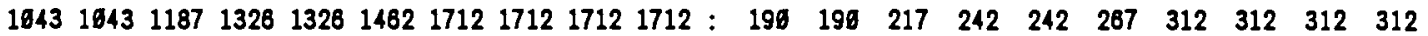
$\begin{array}{lllllllllllllllllllll}696 & 872 & 1017 & 1017 & 1219 & 1219 & 1219 & 1219 & 1219 & 1219 & : & 129 & 161 & 188 & 188 & 228 & 228 & 226 & 226 & 226 & 226\end{array}$ $\begin{array}{lllllllllllllllllllll}822 & 967 & 1131 & 1131 & 1299 & 1437 & 1437 & 1642 & 1751 & 1751 & : & 156 & 178 & 268 & 268 & 237 & 262 & 262 & 366 & 326 & 320\end{array}$

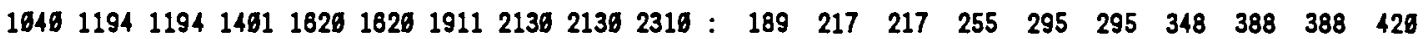
$\begin{array}{lllllllllllllllllllll}248 & 360 & 360 & 360 & 360 & 360 & 360 & 360 & 360 & 360: & 93 & 112 & 112 & 112 & 112 & 112 & 112 & 112 & 112 & 112\end{array}$ $\begin{array}{lllllllllllllllllllll}242 & 242 & 291 & 291 & 351 & 351 & 427 & 486 & 486 & 486 & : & 161 & 161 & 121 & 121 & 146 & 146 & 178 & 262 & 292 & 202\end{array}$ $\begin{array}{lllllllllllllllllllll}170 & 176 & 211 & 211 & 268 & 268 & 268 & 288 & 288 & 268 & : & 79 & 79 & 98 & 98 & 124 & 124 & 124 & 124 & 124 & 124\end{array}$ $\begin{array}{lllllllllllllllllllll}313 & 352 & 418 & 695 & 695 & 695 & 665 & 695 & 695 & 695 & : & 144 & 162 & 191 & 278 & 278 & 278 & 278 & 278 & 278 & 278\end{array}$ $\begin{array}{lllllllllllllllllllll}280 & 326 & 377 & 377 & 456 & 561 & 561 & 569 & 626 & 626 & : & 88 & 169 & 128 & 128 & 152 & 170 & 176 & 192 & 213 & 213\end{array}$ $\begin{array}{lllllllllllllllllllll}253 & 253 & 365 & 357 & 357 & 467 & 486 & 486 & 545 & 693 & : & 98 & 96 & 116 & 135 & 135 & 154 & 184 & 184 & 287 & 229\end{array}$ $\begin{array}{lllllllllllllllllllll}456 & 516 & 623 & 678 & 745 & 861 & 971 & 1632 & 1698 & 1291 & : & 193 & 221 & 267 & 289 & 328 & 343 & 416 & 444 & 471 & 554\end{array}$ $\begin{array}{lllllllllllllllllllll}511 & 577 & 639 & 696 & 771 & 829 & 922 & 995 & 1062 & 1128 & : & 210 & 238 & 285 & 289 & 321 & 346 & 385 & 417 & 445 & 473\end{array}$ $\begin{array}{lllllllllllllllllllll}186 & 186 & 228 & 269 & 269 & 269 & 269 & 269 & 269 & 269 & : & 85 & 85 & 195 & 123 & 123 & 123 & 123 & 123 & 123 & 123\end{array}$ $\begin{array}{lllllllllllllllllllll}540 & 657 & 751 & 842 & 976 & 1684 & 1227 & 1355 & 1471 & 1586 & : & 95 & 116 & 132 & 148 & 172 & 187 & 216 & 239 & 259 & 279\end{array}$

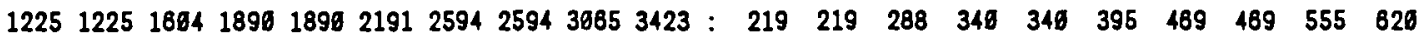
$\begin{array}{lllllllllllllllllllll}186 & 273 & 273 & 338 & 437 & 437 & 561 & 655 & 655 & 741: & 34 & 68 & 56 & 62 & 86 & 89 & 192 & 126 & 126 & 135\end{array}$

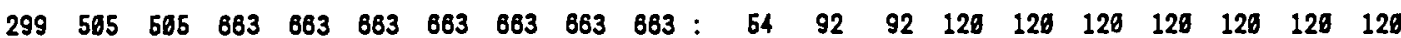
$\begin{array}{lllllllllllllllllllll}342 & 342 & 587 & 587 & 743 & 743 & 743 & 978 & 978 & 1194 & : & 62 & 62 & 92 & 92 & 135 & 135 & 135 & 178 & 178 & 217\end{array}$ $\begin{array}{lllllllllllllllllllll}346 & 346 & 516 & 516 & 748 & 748 & 1636 & 1636 & 1243 & 1243 & 0 & 63 & 63 & 93 & 93 & 136 & 136 & 188 & 188 & 226 & 226\end{array}$ 
TABLE A.8. Middle Case (with Extended Burnup) Maximum At-Reactor Capacity-Plus Transshipment--Projected Cumulative Storage Requirements (contd)

\begin{tabular}{|c|}
\hline POOL \\
\hline VT YANKEE 1 \\
\hline KEIAUNEE \\
\hline BELEONTE 2 \\
\hline SEQUOYAH $1 \mathbf{2}$ \\
\hline WATTS BAR 112 \\
\hline BE—EONTE 1 \\
\hline НАTCH $1 \$ 2$ \\
\hline VOGTLE 1.2 \\
\hline SUSQUEHANNA 122 \\
\hline GINNA \\
\hline RANCHO SECO 1 \\
\hline ORESDEN 3 \\
\hline QUAD CITIES $1 \mathrm{~L}$ \\
\hline LASALE CTY 142 \\
\hline B VALLEY 2 \\
\hline B VALLEY 1 \\
\hline CLINTON 1 \\
\hline SALEX 1 \\
\hline SALEN 2 \\
\hline WNP-1 \\
\hline WNP-3 \\
\hline PALO VERDE 2 \\
\hline PALO VERDE 1 \\
\hline PALO VERDE 3 \\
\hline SUMMER 1 \\
\hline CRYSTAL RVR 3 \\
\hline NINE MILE PT2 \\
\hline RVR BEND 1 \\
\hline WATERFORD 3 \\
\hline SEABROOK-2 \\
\hline SEABROOK 1 \\
\hline HOPE CRK 1 \\
\hline $\begin{array}{l}\text { COMANCHE PK } 112 \\
\text { CAШANAY } 1\end{array}$ \\
\hline
\end{tabular}

Assenblies

NTIHM

\begin{tabular}{|c|c|c|c|c|c|c|c|c|c|c|c|c|c|c|c|c|c|c|c|c|}
\hline 2005 & $\underline{2098}$ & 2687 & 2008 & 26009 & 2010 & 2011 & 2012 & 2013 & 2014 & & 2905 & 2908 & 2987 & $\underline{2998}$ & $\underline{2089}$ & 2010 & 2011 & 2012 & 2013 & 2814 \\
\hline 224 & 291 & 344 & 344 & 344 & 344 & 344 & 344 & 344 & 344 & & 41 & 53 & 83 & 63 & 83 & 63 & 83 & 83 & 63 & 63 \\
\hline 184 & 132 & 154 & 177 & 208 & 268 & 288 & 268 & 208 & 298 & : & 48 & 51 & 59 & 68 & 80 & 80 & 80 & 80 & 86 & 86 \\
\hline 21 & 88 & 157 & 157 & 251 & 297 & 297 & 396 & 445 & 445 & : & 16 & 46 & 72 & 72 & 115 & 136 & 136 & 181 & 263 & 203 \\
\hline 52 & 110 & 157 & 252 & 319 & 365 & 527 & 590 & 850 & 768 & : & 24 & 51 & 72 & 116 & 148 & 188 & 242 & 271 & 298 & 325 \\
\hline 112 & 185 & 220 & 315 & 315 & 468 & 455 & 528 & 639 & 712 & : & 51 & 78 & 101 & 145 & 145 & 188 & 209 & 241 & 293 & 327 \\
\hline \multirow[t]{29}{*}{58} & 58 & 127 & 178 & 178 & 223 & 223 & 311 & 380 & 360 & : & 28 & 28 & 58 & 81 & 81 & 162 & 162 & 142 & 164 & 164 \\
\hline & 127 & 280 & 410 & 627 & 757 & 1019 & * 1223 & 1418 & 1978 & : & & 23 & 51 & 75 & 114 & 138 & 186 & 223 & 258 & 360 \\
\hline & 36 & 134 & 262 & 324 & 388 & 477 & 667 & 675 & 793 & : & & 17 & 62 & 93 & 149 & 179 & 220 & 280 & 311 & 366 \\
\hline & 138 & 415 & 550 & 752 & 1016 & 1255 & 1450 & 1795 & 1970 & : & & 24 & 73 & 97 & 132 & 179 & 221 & 255 & 316 & 346 \\
\hline & & 9 & 9 & 9 & 9 & 9 & 9 & 9 & 9 & : & & 3 & 3 & 3 & 3 & 3 & 3 & 3 & 3 & 3 \\
\hline & 2 & 41 & 72 & 72 & 169 & 169 & 169 & 215 & 215 & : & & 1 & 19 & 33 & 33 & 50 & 78. & 78 & 99 & 99 \\
\hline & & 10 & 10 & 16 & 10 & 16 & 10 & 10 & 10 & : & & & 2 & 2 & 2 & 2 & 2 & 2 & 2 & 2 \\
\hline & & 181 & 201 & 925 & 925 & 925 & 925 & 925 & 925 & : & & & 18 & 35 & 163 & 163 & 163 & 163 & 163 & 163 \\
\hline & & 132 & 261 & 634 & 761 & 993 & 1348 & 1569 & 1668 & : & & & 24 & 48 & 115 & 139 & 181 & 246 & 275 & 304 \\
\hline & & 54 & 54 & 110 & 146 & 145 & 211 & 249 & 249 & : & & & 25 & 25 & 51 & 67 & 67 & 97 & 115 & 115 \\
\hline & & & 36 & 36 & 72 & 147 & 147 & 200 & 252 & & & & & 17 & 17 & 33 & 68 & 88 & 92 & 118 \\
\hline & & 37 & 153 & 153 & 272 & 416 & 416 & 594 & 796 & ; & & & 7 & 28 & 28 & 50 & 76 & 76 & 109 & 144 \\
\hline & & 31 & 77 & 77 & 122 & 198 & 198 & 253 & 367 & : & & & 14 & 35 & 35 & 56 & 91 & 91 & 116 & 141 \\
\hline & & 48 & 46 & 169 & 155 & 155 & 216 & 271 & 271 & * & & & 21 & 21 & 58 & 71 & 71 & 99 & 124 & 124 \\
\hline & & 18 & 53 & 104 & 139 & 193 & 263 & 326 & 370 & . & & & 8 & 24 & 47 & 63 & 88 & 120 & 148 & 169 \\
\hline & & 49 & 82 & 135 & 187 & 222 & 293 & 351 & 484 & : & & & 21 & 35 & 58 & 72 & 98 & 126 & 151 & 174 \\
\hline & & & & 35 & 72 & 112 & 170 & 225 & 260 & & & & & & 15 & 31 & 49 & 74 & 98 & 113 \\
\hline & & & & & 37 & 77 & 135 & 196 & 224 & . & & & & & & 16 & 34 & 59 & 83 & 98 \\
\hline & & & & 60 & 95 & 139 & 298 & 234 & 293 & & & & & & 26 & 41 & 81 & 87 & 162 & 128 \\
\hline & & & & & 29 & 28 & 77 & 122 & 175 & & & & & & & 9 & 9 & 35 & 58 & 80 \\
\hline & & & & & & 9 & 9 & 49 & 89 & & & & & & & & 4 & 4 & 23 & 41 \\
\hline & & & & & & 78 & 343 & 343 & 482 & & & & & & & & 14 & 62 & 62 & 87 \\
\hline & & & & & & & 118 & 248 & 317 & & & & & & & & & 22 & 44 & 58 \\
\hline & & & & & & & 42 & 42 & 124 & & & & & & & & & 18 & 18 & 53 \\
\hline & & & & & & & 49 & 108 & 156 & & & & & & & & & 21 & 45 & 66 \\
\hline & & & & & & & & 53 & 86 & & & & & & & & & & 22 & 38 \\
\hline & & & & & & & 184 & 325 & 325 & & & & & & & & & 33 & 59 & 59 \\
\hline & & & & & & & & 36 & 167 & & & & & & & & & & 15 & 43 \\
\hline & & & & & & & & & 37 & & & & & & & & & & & 18 \\
\hline
\end{tabular}


TABLE A.8. Middle Case (with Extended Burnup) Maximum At-Reactor Capacity-Plus Transshipment--Projected Cumulative Storage Requirements (contd)

Assemblies

MTIHM

POOL

PIR TOTAL

BWR TOTAL

TOTAL

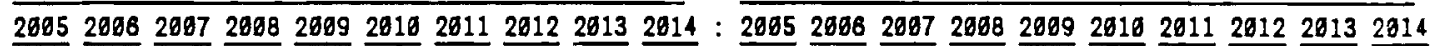

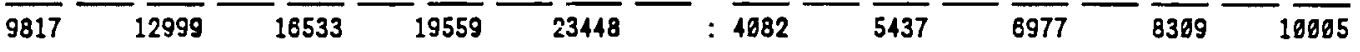
$11863 \quad 14473 \quad 17887 \quad 21521 \quad 25373:$ $\begin{array}{lllllllllllllll}15208 & 19328 & 24308 & 28691 & 33873 & : & 2741 & 3489 & 4389 & 5184 & 6124 & \\ 16679 & 20975 & 26049 & 31439 & 38670 & : & 3009 & 3788 & 4703 & 5682 & 6632\end{array}$

$\begin{array}{llllllllll}25023 & 32327 & 40841 & 48250 & 57321 & : 6823 & 8926 & 11366 & 13498 & 16129\end{array}$ $\begin{array}{llllllllll}27742 & 35448 & 43907 & 52960 & 62943: & 7613 & 9875 & 12266 & 14844 & 17439\end{array}$ 
TABLE A.8. Middle Case (with Extended Burnup) Maximum At-Reactor Capacity-Plus Transshipment--Projected Cumulative Storage Requirements (contd)

\begin{tabular}{|c|c|c|c|c|c|c|c|c|c|c|c|c|c|}
\hline \multirow[b]{2}{*}{ POOL } & \multicolumn{6}{|c|}{ Assemblies } & \multicolumn{7}{|c|}{ MTIHM } \\
\hline & 15 & $\underline{2016}$ & 2017 & 2018 & $\underline{2019}$ & 2820 & & 2915 & 2018 & 2917 & 2018 & 2819 & 202 \\
\hline IUSTONE 1 & 70 & 1470 & 1470 & 1470 & 1478 & 1470 & & 81 & 261 & 261 & 261 & 61 & 261 \\
\hline ALISADES & 363 & 603 & 683 & 683 & 683 & 603 & & 235 & 235 & 235 & 235 & 35 & 235 \\
\hline RAIRIE ISL & 942 & 942 & 942 & 942 & 942 & 942 & & 330 & 330 & 330 & 330 & 30 & 330 \\
\hline [LGRIM 1 & 293 & 1293 & 1293 & 1293 & 1293 & 1293 & & 229 & 229 & 229 & 29 & 29 & 29 \\
\hline RUNSWICK 2 & 11 & 1811 & 1811 & 1811 & 1811 & 1811 & & 339 & 339 & 339 & 339 & 39 & 339 \\
\hline RUNSWICK 1 & 37 & 1937 & 1937 & 1937 & 1937 & 1937 & & 362 & 362 & 362 & 362 & 62 & 62 \\
\hline ALYERT CLF & 25 & 1725 & 1725 & 1725 & 1725 & 1725 & & 647 & 647 & 647 & 647 & 647 & 647 \\
\hline CROS & 66 & 168 & 188 & 168 & 168 & 168 & & 8 & 18 & 18 & 18 & 8 & 18 \\
\hline TZPATRICX & 159 & 1859 & 1859 & 1859 & 1859 & 1859 & & 34 & 334 & 334 & 334 & 34 & 34 \\
\hline NOIAN PT 3 & 658 & 720 & 720 & 720 & 720 & 720 & & 360 & 328 & 328 & 328 & 28 & 28 \\
\hline JRRY $1 \mathbf{2}$ & 12 & 912 & 912 & 912 & 912 & 912 & & 419 & 419 & 419 & 419 & 9 & 18 \\
\hline ORTH ANNA 1 & 1475 & 1538 & 1695 & 1895 & 1695 & 1695 & & 681 & 710 & 782 & 782 & 82 & 782 \\
\hline DPER STN & 778 & 778 & 778 & 778 & 778 & 778 & & 142 & 142 & 142 & 142 & 42 & 142 \\
\hline NE 3 & 793 & 833 & 871 & 871 & 985 & 982 & & 8 & 84 & 482 & 402 & 8 & 44 \\
\hline NODAM NECX & 272 & 272 & 272 & 272 & 272 & 272 & & 112 & 112 & 112 & 112 & 12 & 112 \\
\hline ILSTONE 2 & 839 & 639 & 639 & 639 & 639 & 639 & $\theta$ & 259 & 259 & 259 & 259 & 9 & 59 \\
\hline LHOUN & 1 & 311 & 311 & 1 & 11 & 11 & & 2 & 12 & 112 & 112 & 2 & 12 \\
\hline AVIS-BESSE 1 & 98 & 658 & 714 & 714 & 714 & 714 & & 280 & 188 & 334 & 334 & 34 & 334 \\
\hline RAIDWOOD 182 & 141 & 1230 & 1302 & 1368 & 1428 & 1530 & • & 482 & 520 & 550 & 578 & 83 & 347 \\
\hline IRROL COUN & 34 & 812 & 894 & 8 & 1 & 1126 & & $\theta$ & 3 & 378 & 12 & 10 & 476 \\
\hline ION & 50 & 750 & 750 & 750 & 750 & 750 & & 343 & 343 & 343 & 343 & 3 & 343 \\
\hline YYRON 122 & .02 & 1191 & 1262 & 1321 & 1383 & 1485 & : & 466 & 504 & 534 & 559 & 885 & 28 \\
\hline$x 1$ & g & 70 & 6 & 10 & 70 & 70 & • & $y$ & $y$ & 9 & 9 & $y$ & 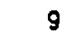 \\
\hline OINT BEACH & 26 & 628 & 626 & 626 & 828 & 626 & & 222 & 222 & 222 & 222 & 22 & 22 \\
\hline NRICO FERMI2 & 2112 & 2345 & 2495 & 2495 & 2850 & 2650 & : & 384 & 427 & 454 & 454 & 482 & 482 \\
\hline JYSTER CRK 1 & 525 & 525 & 525 & 525 & 525 & 525 & ： & 3 & 3 & 93 & 93 & 93 & 93 \\
\hline 'EACHBOTTOM 3 & 2 & 2 & 1712 & 1712 & 1712 & 1712 & & 2 & 312 & 312 & 312 & 2 & 31 \\
\hline 'EACHBOTTON 2 & 1219 & 1219 & 1219 & 1219 & 1219 & 1219 & : & 226 & 226 & 226 & 226 & 228 & 228 \\
\hline IMERICX 1 & 19 & 2054 & 2054 & 2179 & 2333 & 2333 & : & 350 & 375 & 375 & 398 & 426 & 426 \\
\hline -IMERICK 2 & 2523 & 2523 & 2730 & 2949 & 2949 & 3148 & : & 459 & 459 & 497 & 537 & 37 & 57 \\
\hline WAINE YANKEE & 300 & 360 & 380 & 300 & 390 & 360 & : & 112 & 112 & 112 & 112 & 112 & 112 \\
\hline LEAR 2 & 88 & 486 & 486 & 488 & 486 & 486 & r & 202 & 282 & 202 & 202 & 82 & 202 \\
\hline ARK NUCLEAR 1 & 268 & 268 & 268 & 288 & 288 & 268 & : & 124 & 124 & 124 & 124 & 124 & 124 \\
\hline TURKEY PT $1 \& 2$ & 665 & 605 & 895 & 685 & 685 & 685 & : & 278 & 278 & 278 & 278 & 278 & 278 \\
\hline E 1 & 6 & 26 & 826 & 626 & 828 & 826 & : & & 213 & 213 & 213 & 13 & 213 \\
\hline ST LUCIE 2 & 663 & 674 & 740 & 740 & 788 & 844 & $:$ & 229 & 256 & 281 & 281 & 299 & 328 \\
\hline $\operatorname{cosk} 122$ & 291 & 1291 & 1291 & 1291 & 1291 & 1291 & $:$ & 554 & 554 & 554 & 554 & 554 & 554 \\
\hline SAN ONOFRE $1,2,13$ & 1185 & 1395 & 1395 & 1438 & 1438 & 1577 & $:$ & 498 & 549 & 549 & 605 & 685 & 865 \\
\hline INDIAN PT 2 & 269 & 269 & 289 & 269 & 269 & 269 & $:$ & 123 & 123 & 123 & 123 & 123 & 123 \\
\hline MASH NUCLEAR2 & 1671 & 1812 & 1945 & 2045 & 2137 & 2247 & . & 294 & 319 & 343 & 360 & 378 & 396 \\
\hline PERRY 112 & 3423 & 3842 & 4223 & 4223 & 4542 & 4994 & : & 620 & 698 & 768 & 768 & 824 & 986 \\
\hline DUANE ARNOLD & 741 & 741 & 741 & 741 & 741 & 741 & : & 135 & 135 & 135 & 135 & 135 & 135 \\
\hline BROWNS FERRY1 & 863 & 863 & 863 & 863 & 683 & 663 & : & 129 & 120 & 120 & 126 & $12 \varnothing$ & 129 \\
\hline BROTNS FERRY2 & 194 & 1194 & 1194 & 1194 & 1194 & 1194 & : & 217 & 217 & 217 & 217 & 217 & 217 \\
\hline BROWNS FERRY3 & 1388 & 1388 & 1622 & 1822 & 1622 & 1622 & 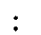 & 252 & 252 & 294 & 294 & 294 & 294 \\
\hline
\end{tabular}


TABLE A.8. Middle Case (with Extended Burnup) Maximum At-Reactor Capacity-Plus Transshipment--Projected Cumulative Storage Requirements (contd)

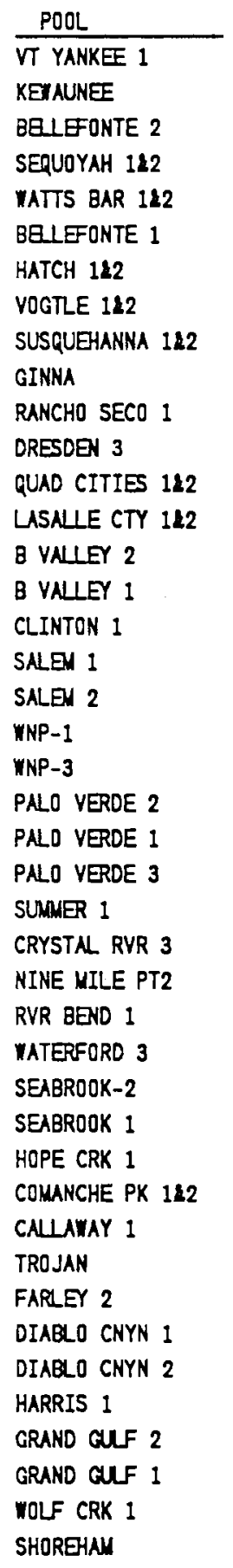

\begin{tabular}{|c|c|c|c|c|c|c|c|c|c|c|c|c|}
\hline \multicolumn{6}{|c|}{ Assemblies } & \multicolumn{7}{|c|}{ MTIHM } \\
\hline 2015 & 2016 & 2017 & 2618 & 2019 & 2620 & : & 2915 & 2018 & $\underline{2017}$ & 2018 & 2019 & $\underline{2920}$ \\
\hline 344 & 344 & 344 & 344 & 344 & 344 & : & 63 & 63 & 63 & 83 & 83 & 63 \\
\hline 268 & 288 & 208 & 208 & 208 & 208 & : & 80 & 80 & 80 & 80 & 80 & 80 \\
\hline 512 & 512 & 572 & 837 & 637 & 695 & : & 234 & 234 & 281 & 291 & 291 & 317 \\
\hline 750 & 888 & 933 & 984 & 1074 & 1674 & : & 344 & 468 & 428 & 452 & 493 & 493 \\
\hline 773 & 885 & 885 & 984 & 1628 & 1697 & : & 355 & 486 & 406 & 452 & 472 & 504 \\
\hline 433 & 492 & 492 & 547 & $B 13$ & 813 & : & 198 & 224 & 224 & 250 & 280 & 280 \\
\hline 1976 & 1978 & 1976 & 1978 & 1976 & 1976 & $:$ & 360 & 360 & 350 & 360 & 360 & 360 \\
\hline 882 & 962 & 1026 & 1110 & 1201 & 1280 & : & 467 & 444 & 473 & 512 & 554 & 591 \\
\hline 2094 & 2515 & 2799 & 2859 & 3131 & 3300 & : & 368 & 442 & 476 & 503 & 550 & 580 \\
\hline & 9 & 9 & 9 & 9 & 9 & $:$ & 3 & 3 & 3 & 3 & 3 & 3 \\
\hline 215 & 215 & 215 & 215 & 215 & 215 & $:$ & 99 & 99 & 99 & 99 & 99 & 99 \\
\hline 10 & 16 & 16 & 16 & 10 & 10 & : & 2 & 2 & 2 & 2 & 2 & 2 \\
\hline 925 & 925 & 925 & 925 & 925 & 925 & : & 183 & 183 & 183 & 163 & 163 & 163 \\
\hline 1982 & 2097 & 2281 & 2564 & 2888 & 2878 & : & 348 & 382 & 415 & 467 & 498 & 524 \\
\hline 395 & 351 & 351 & 387 & 439 & 439 & $\therefore$ & 141 & 182 & 162 & 178 & 292 & 202 \\
\hline 252 & 252 & 252 & 252 & 252 & 252 & : & 118 & 118 & 116 & 118 & 118 & 116 \\
\hline 790 & 949 & 1081 & 1961 & 1177 & 1397 & $:$ & 144 & 173 & 194 & 194 & 215 & 239 \\
\hline 307 & 375 & 439 & 439 & 439 & 439 & : & 141 & 172 & 202 & 262 & 202 & 282 \\
\hline 314 & 381 & 381 & 431 & 487 & 487 & : & 144 & 175 & 175 & 198 & 224 & 224 \\
\hline 411 & 459 & 568 & 557 & 597 & 648 & : & 187 & 289 & 232 & 254 & 272 & 296 \\
\hline 447 & 497 & 548 & 599 & B41 & 694 & : & 193 & 214 & 237 & 259 & 277 & 360 \\
\hline 307 & 357 & 397 & 435 & 471 & 529 & : & 134 & 156 & 173 & 196 & 208 & 231 \\
\hline 273 & 324 & 365 & 401 & 437 & 495 & : & 119 & 141 & 159 & 175 & 191 & 218 \\
\hline 337 & 377 & 411 & 453 & 499 & 538 & : & 147 & 184 & 179 & 198 & 218 & 235 \\
\hline 200 & 200 & 261 & 300 & 338 & 381 & : & 92 & 92 & 128 & 138 & 155 & 175 \\
\hline 89 & 139 & 184 & 184 & 184 & 184 & : & 41 & 84 & 85 & 85 & 85 & 85 \\
\hline 482 & 898 & 698 & 848 & 1034 & 1034 & : & 87 & 125 & 125 & 152 & 188 & 188 \\
\hline 429 & 549 & 644 & 732 & 815 & 958 & : & 78 & 100 & 117 & 133 & 149 & 173 \\
\hline 167 & 211 & 251 & 292 & 292 & 345 & : & 71 & 90 & 187 & 125 & 125 & 147 \\
\hline 197 & 245 & 294 & 343 & 383 & 434 & : & 83 & 104 & 124 & 145 & 162 & 184 \\
\hline 134 & 186 & 227 & 257 & 291 & 347 & $:$ & 57 & 79 & 98 & 169 & 123 & 147 \\
\hline 528 & 898 & 898 & 850 & 1039 & 1039 & : & 98 & 127 & 127 & 155 & 189 & 189 \\
\hline 268 & 368 & 392 & 483 & 536 & 854 & : & 83 & 124 & 158 & 187 & 216 & 264 \\
\hline 37 & 111 & 177 & 177 & $22 B$ & 283 & : & 16 & 47 & 75 & 75 & 96 & 120 \\
\hline 10 & 10 & 10 & 10 & 10 & 10 & : & 5 & 5 & 5 & 5 & 5 & 5 \\
\hline & 40 & 40 & 40 & 40 & 40 & : & $\theta$ & 18 & 18 & 18 & 18 & 18 \\
\hline & 28 & 79 & 79 & 108 & 158 & : & $\theta$ & 13 & 36 & 38 & 50 & 72 \\
\hline & & 41 & 70 & 120 & 158 & : & & & 19 & 32 & 55 & 72 \\
\hline & & & 19 & 55 & 55 & $\cdot$ & & & & 9 & 26 & 26 \\
\hline & & & 33 & 169 & 340 & : & & & & 8 & 31 & 82 \\
\hline & & & & 151 & 324 & : & & & & & 27 & 57 \\
\hline & & & & & 15 & $\theta^{\circ}$ & & & & & & 7 \\
\hline & & & & & 77 & 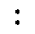 & & & & & & 14 \\
\hline
\end{tabular}


TABLE A.8. Middle Case (with Extended Burnup) Maximum At-Reactor Capacity-Plus Transshipment--Projected Cumulative Storage Requirements (contd)

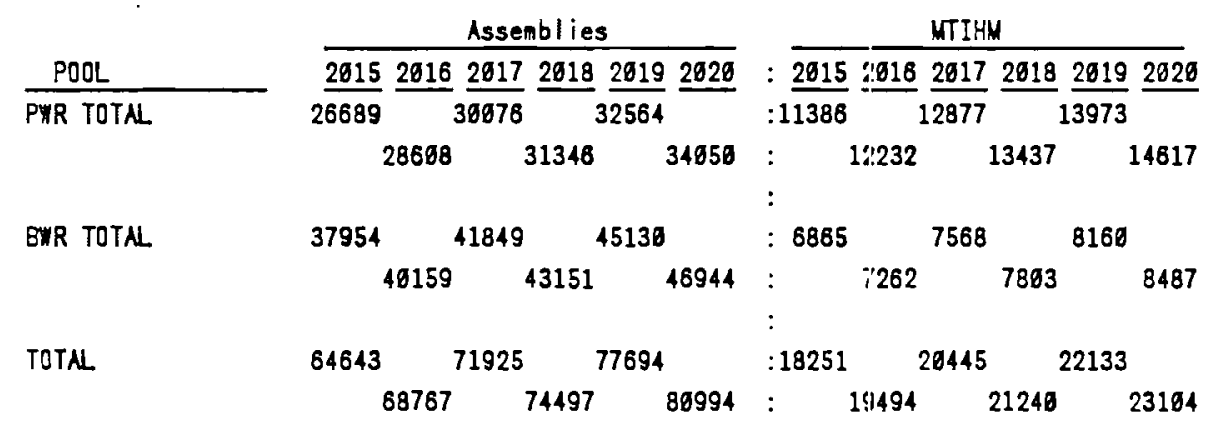


APPENDIX B

MAP OF FEDERAL ENERGY REGIONS 



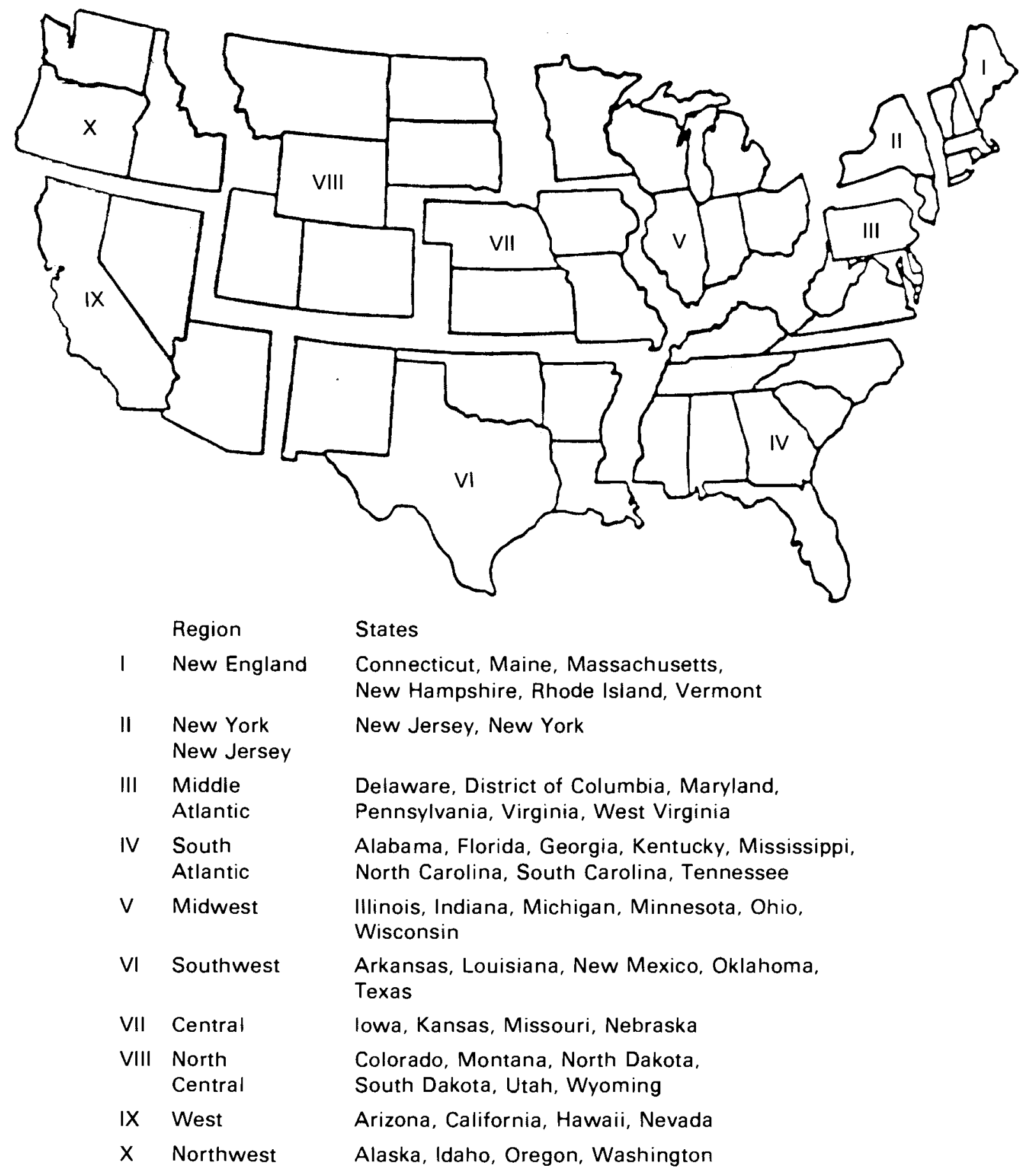

FIGURE B.1. Federat Energy Regions 


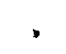




\section{DISTRIBUTION}

No. of

Copies

OFFSITE

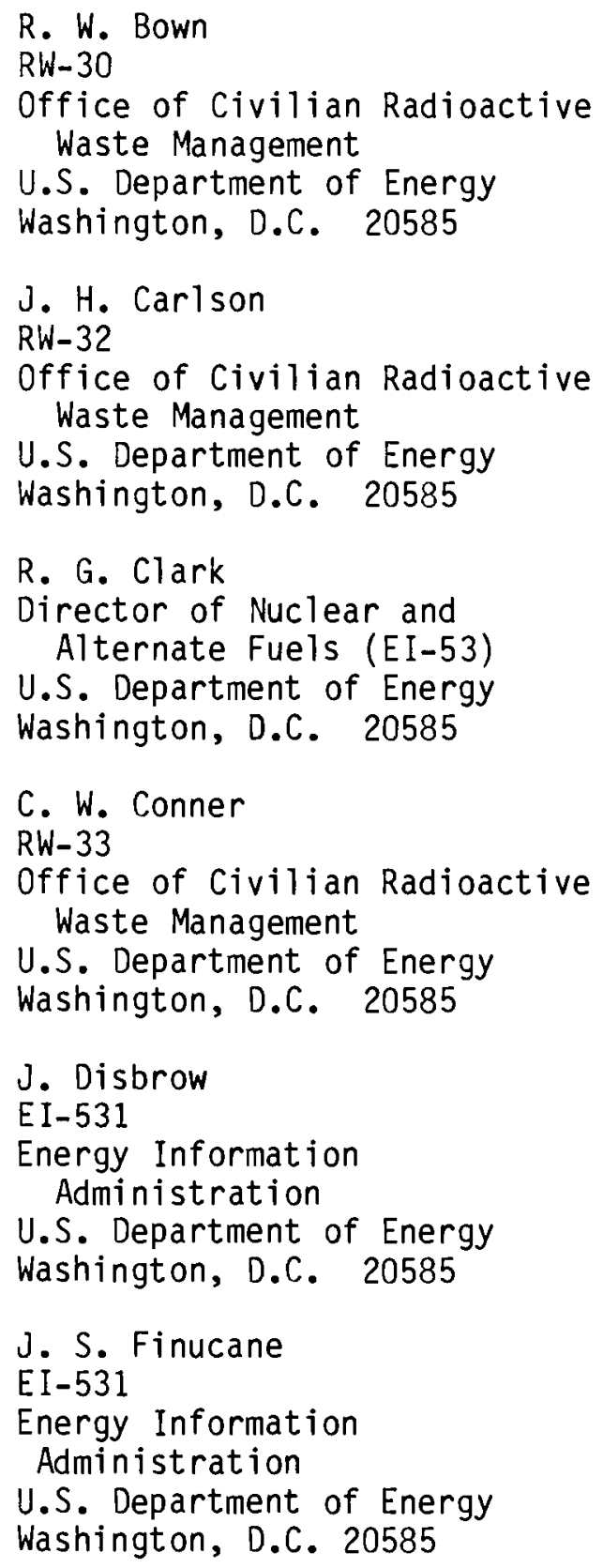

R. W. Bown

$\mathrm{RW}-30$

Office of Civilian Radioactive Waste Management

U.S. Department of Energy

Washington, D.C. 20585

J. H. Carlson

$\mathrm{RW}-32$

Office of Civilian Radioactive Waste Management

U.S. Department of Energy

Washington, D.C. 20585

R. G. Clark

Director of Nuclear and

Alternate Fuels (EI-53)

U.S. Department of Energy

Washington, D.C. 20585

C. W. Conner

RW-33

Office of Civilian Radioactive Waste Management

U.S. Department of Energy

Washington, D.C. 20585

No. of

Copies

W. A. Frankhauser

DP-122.1

U.S. Department of Energy

Washington, D.C. 20545

J. R. Hilley

$\mathrm{RW}-30$

Office of Civilian Radioactive Waste Management

U.S. Department of Energy

Washington, D.C. 20585

T. H. Isaacs

$\mathrm{RW}-42$

Office of Civilian Radioactive Waste Management

U.S. Department of Energy

Washington, D.C. 20585

K. A. Klein

$\mathrm{RW}-32$

Office of Civilian Radioactive Waste Management

U.S. Department of Energy

Washington, D.C. 20585

E. F. Mastel

NE-72

U.S. Department of Energy

Washington, D.C. 20545

20 R. A. Milner

$\mathrm{RW}-12$

U.S. Department of Energy

Washington, D.C. 20545

T. D. Nguyen

RW-33

Office of Civilian Radioactive Waste Management

U.S. Department of Energy

Washington, D.C. 20585 
No. of

Copies

M. L. Payton

$\mathrm{RW}-12$

Office of Civilian Radioactive Waste Management

U.S. Department of Energy

Washington, D.C. 20585

R. Philpott

$\mathrm{RW}-12$

Office of Civilian Radioactive Waste Management

U.S. Department of Energy

Washington, D.C. 20585

D. E. Shelor

$\mathrm{RW}-32$

Office of Civilian Radioactive Waste Management

U.S. Department of Energy

Washington, D.C. 20585

H. Steinberg

RW-33

Office of Civilian Radioactive Waste Management

U.S. Department of Energy

Washington, D.C. 20585

W. M. Sprecher

$\mathrm{RW}-42$

Office of Civilian Radioactive Waste Management

U.S. Department of Energy

Washington, D.C. 20585

E. L. Wilmot

$\mathrm{RW}-33$

Office of Civilian Radioactive Waste Management

U.S. Department of Energy

Washington, D.C. 20585

R. C. Ashline

Oak Ridge National

Laboratory

P.0. Box X

Oak Ridge, TN 37830
No. of

Copies

\author{
C. W. Forsberg \\ Oak Ridge National \\ Laboratory \\ P.0. Box $X$ \\ Oak Ridge, TN 37830
}

J. A. Klein

Oak Ridge National Laboratory

P.0. Box X

Oak Ridge, TN 37830

D. S. Joy

Oak Ridge National Laboratory

P.0. BCIX X

Oak Ricge, TN 37830

S. N. Storch

Oak Riclge National Laboratory

P.0. Box X

Oak Ridge, TN 37830

Ken Golleher

DOE Albuquergue Operations Office

P. O. Box 5400

Albuquerque, NM 87115

G. C. Allen

Sandia National Laboratories

P.0. Box 5800

A7buquerque, NM 87185

J. W. Cashwell

Sandia National Laboratories

P.0. Box 5800

Albuquerque, NM 87185

E. R. Johnson

JAI

11702 Bowman Green

Reston, VA 22090 
No. of

Copies

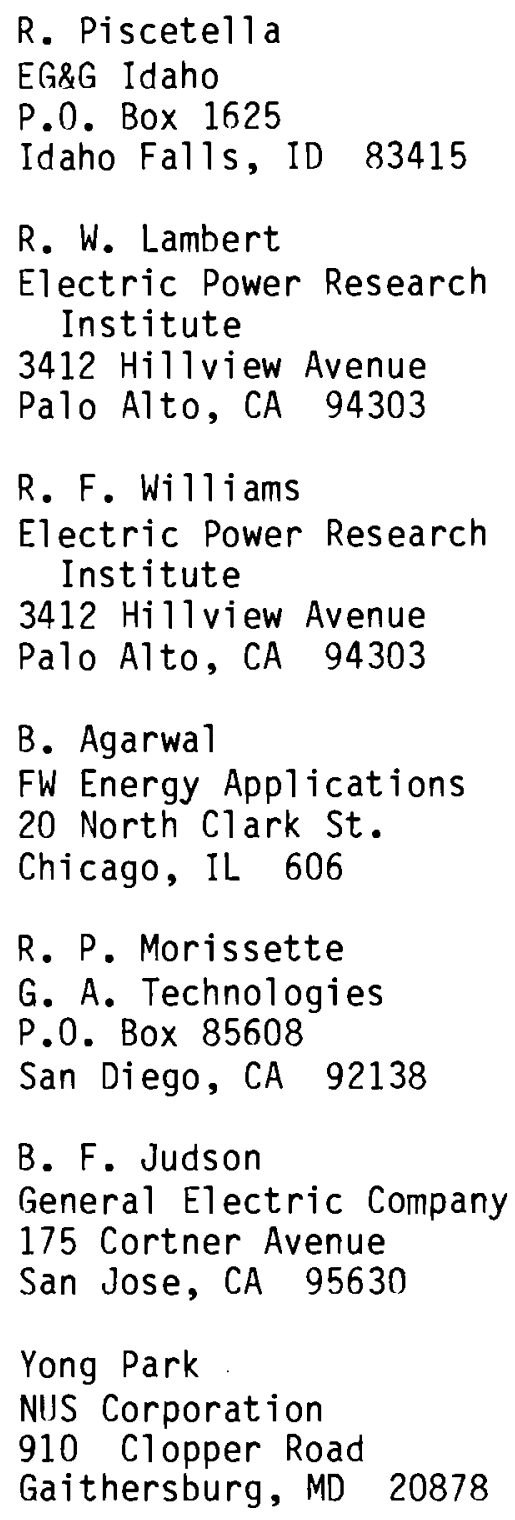

R. Piscetella

EG\&G Idaho

P.0. Box 1625

Idaho Falls, ID 83415

R. W. Lambert

Electric Power Research Institute

3412 Hillview Avenue

Palo Alto, CA 94303

R. F. Williams

Electric Power Research Institute

$3412 \mathrm{Hill}$ lview Avenue

Palo Alto, CA 94303

B. Agarwal

FW Energy Applications

20 North Clark St.

Chicago, IL 606

R. P. Morissette

G. A. Technologies

P.0. Box 85608

San Diego, CA 92138

B. F. Judson

General Electric Company

175 Cortner Avenue

San Jose, CA 95630

Yong Park

NUS Corporation

910 Clopper Road

Gaithersburg, MD 20878

B. R. Teer

Transnuclear, Inc.

One North Broadway

White Plains, NY 10601

Edward Kuhn

Stone \& Webster Engineering Corp.

One Penn Plaza

250 West 34th Street

New York, NY 10119
No. of

Copies

Ralph Best

7938 Sarahurst

Dublin, $\mathrm{OH} 43017$

J. H. Saling

Westinghouse Electric Corp.

Waste Technology Service

Division

Headquarters Site

P.0. Box 286

Madison, PA 15663

4 Frank Haines

Roy $F$. Weston

955 L'Enfant Plaza S. W. 8th Floor

Washington, DC 20585

S. K. Gupta

Battelle Project Management Division

505 King Ave.

Columbus, $\mathrm{OH}$ 43201-2693

30 DOE Technical Information

Center

ONSITE

6 DOE Richland Operations Office

D. E. Crouter

R. D. Izatt (2)

J. M. Peterson

D. C. Langstaff

J. J. Sutey

70 Pacific Northwest Laboratory

M. K. Altenhofen

W. J. Bailey

G. H. Beeman

A. J. Boegel

J. L. Braitman

L. L. Clark

B. M. Cole

C. A. Counts

J. W. Currie 
No. of

Copies

P. M. Daling

L. R. Dodd

A. L. Doherty

K. A. Ekblaw

R. L. Engel

J. F. Fletcher

C. M. Heeb (10)

J. N. Holl loway

G. M. Holter

C. J. Hostick

C. H. Imhoff

A. B. Johnson

G. J. Konzek

R. A. Libby

W. W. Little

A. T. Luksic

R. W. McKee

G. W. McNair

D. F. Newman
No. of

Copies

D. R. Oden

P. T. Ot is

W. L. Purcell

R. G. Rau

J. M. Reilly

F. P. Roberts

N. L. 'scharnhorst

K. J. schneider

M. R. 'shay

S. M. short

D. J. Silviera

R. I. smith

M. B. Triplett

R. C. Nalling (10)

M. K. White

T. W. Nood

J. R. Young

Publisning Coordination MH (2)

Technical Information (5) 